\title{
Final Report \\ "SULFUR RELEASE FROM OHIO COALS \\ AND SORBENT KINETICS IN PULVERIZED COAL FLAMES"
}

Project $C D O / R-87-93$

Robert Essenhigh

The ohio state University

Mechanical Engineering

Robinson Laboratory

206 W. 18th Avenue

Columbus, $\mathrm{OH} 43210$

614/292-0403

Date of Submission: August, 1992

This project was funded in part by the ohio coal Development office, Department of Development, state of ohio 
FINAL REPORT

on

SULFUR RELEASE FROM OHIO COAIS

AND SORBENT KINETICS IN PULVERIZED COAL FLAMES

\section{Project Manager:}

Robert. H. Essenhigh, E.G. Bailey Professor of Energy Conversion

Department of Mechanical Engineering

The Ohio State University

206 West 18th. Avenue

Columbus, OH. 43210

(614) 292-0403

Project Support:

(1) This project was funded in part by the Ohio coal Development Office, Department of Development, State of Ohio Project No: CDO/R-87-93

(2) Additional support was provided by The ohio State University Department of Mechanical Engineering Combustion Laboratory Industrial Fund

Project Investigators:

Ann M. Mescher; Graduate Research Associate

Department of Mechanical Engineering, The Ohio State University

Xuedong Zhu; Graduate Research Associate

Department of Mechanical Engineering, The Ohio State University

Dr. Marian w. Siudek; Visiting Scholar in Mechanical Engineering Department of Mechanical Engineering, The Ohio State University

Date of Submission:

August, 1992

Combustion Laboratoly

Department of Mechanical Engineering

The Ohio State University

Columbus, $\mathrm{OH} 43210$ 


\section{ACKNOWLEDGEMENIS}

This work was supported principally by The Ohio Coal Development Office, state of Ohio (J. Bird, Director) with additional financial support from: industrial contributors to the Department of Mechanical Engineering Combustion Laboratory Industrial fund and from The Ohio State University. We also gratefully acknowledge the consolidation coal company through the agency of Dr. Robert Douglas for the donation of coal supplies for this work. 
TABLE OF CONTENIS

VOLUME I

Page

PART I: EXECUTTVE SUMMARY ............... 1

PART II: TECHNICAL REPORT

1. INTRODUCTION . . . . . . . . . . . . . . 1.1

2. EXPERTMENTAL ..................... 2.1

2.1 Furnace . . . . . . . . . . . . . . . 2.1

2.2 Material Supplies ................. 2.3

2.3 Gas Sampling and Analysis . . . . . . . . . . . 2.9

2.4 Solid Sampling and Analysis . . . . . . . . . . . 2.11

2.5 Temperature and Velocity Measurements . . . . . . . 2.14

2.6 Data Compilation and Reduction........... 2.21

2.7 Coal and Limestone Sources . . . . . . . . . . . 2.22

Table 2.1 . . . . . . . . . . . . . . . 2.24

Figures 2.1 through 2.5 following . . . . . . . . . 2.24

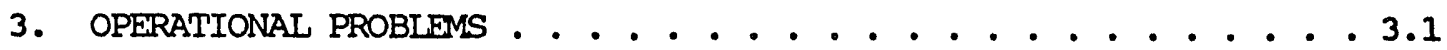

4. RESULTS I: THEORY . . . . . . . . . . . . . 4.1

4.1 Physical Model .................... 4.1

4.2 Mathematical Model .. . . . . . . . . . . . 4.5

4.3 Solution Procedure . . . . . . . . . . . . . 4.12

4.4 Selected Results . . . . . . . . . . . . . . . 4.15

Figures 4.1 through 4.4 following . . . . . . . . . . 4.22

5. RESULTS II: DEVELOPMENT OF SAMPLING AND ANALYSIS TECHNIQUES • 5.1

5.1 Sampling Procedures . . . . . . . . . . . . . 5.1 5.2 Effect of Solid Sample Handling on PSD Measurements . . . 5.3

5.3 Probe Design ............... . . . 5.7

5.4 PSD Analysis of Coal/Limestone Samples . . . . . . . . 5.9

5.5 Proximate Analysis of Samples Containing Limestone . . . 5.12

Table 5.1 ..................... 5.18

Figures 5.1 through 5.6 following . . . . . . . . 5.18

6. RESULTS III: FLOW STRUCTURE INVESTIGATIONS . . . . . . . . 6.1

6.1 Cold Flow profiles................. 6.1

6.2 Hot Flow Profiles .................. 6.14

6.3 Summary of Conclusions . . . . . . . . . . . . 6.19

Table 6.1 . . . . . . . . . . . . . . . . . 6.21

Figures 6.1 through 6.15 following . . . . . . . . . . 6.21 


\section{TABLE OF CONIENTS}

(continued)

Page

7. RESUITS IV: FLAME STRUCTURE INVESTIGATIONS . . . . . . . 7.1

7.1 General character of the Flames . . . . . . . . . 7.2

7.2 Temperature Profile ................. 7.4

7.3 Particle Size Distribution . . . . . . . . . . 7.8

$7.4 \mathrm{SO2}$ Concentration ................ 7.11

7.5 Gas Analysis Reaction Efficiency and Local Excess Air . 7.13

7.6 Proximate Analysis Reaction Efficiency . . . . . . . 7.16

7.7 Horizontal Profiles . . . . . . . . . . . . . 7.18

Figures 7.1 through 7.44 following . . . . . . . . . 7.24

8. EVALUATION AND CONCLUSIONS . . . . . . . . . . . 8.1

\section{VOLUME II}

COAL ANALYSES

VOLUME III

1-D FURNACE DATA FROM 1-91 TO 2-92

VOLUME IV

1-D FURNACE DATA PRTOR TO 1-91

VOLUME V

HIGH INTENSITY FURNACE DATA

VOLUME VI

2-COLOR PYROMETER DATA

VOLUME VII

ADDITIONAL DATA 


\section{EXECUTIVE SUMMARY}

In this Report we describe the results of investigations into the structure of combustion and sulfur release profiles from coal burning in one-Dimensional P.C. flames using a furnace of unique design for the measurements. Selected measurements were also carried out in a special high-intensity furnace also of unique design. The formal project work started in late Fall 1989, with unfunded preliminary work in the months prior to that.

The process of limestone injection into the flame to control sulfur oxides emissions is a long-standing concept that has been given particular formulation in the LIMB process, and studies of such systems provide bases for commercial system economics. Problems with LIMB and related systems indicated need for better understanding of, jointly, the sulfur release from the coal and the sorbent behavior by the limestone.

The investigations as reported in Vol. 1 of this Report used 14 different coals under a range of different initial and operating conditions, and the resulting measurements have provided a data base of major proportions, as tabulated in the attached Vols. 2, 3, 4, 5, 6, and 7 of this Report. This data base consists of sets of measurements totalling about 45000 entries for all independent and dependent parameters involved. The independent parameters included: coal type (analysis), firing rate, stoichiometry (fuel/air ratio), and sorbent content of the 
coal/sorbent mixture where relevant. The dependent parameters for station measurements in profiles down the flames included variously: gas analyses $\left(\mathrm{O}_{2}, \mathrm{CO}_{2}, \mathrm{CO}\right.$, and $\left.\mathrm{SO}_{2}\right)$, solid samples (proximate) analyses, flame temperatures (mostly of the particles by 2-color pyrometry), and particle size distributions (PSD'S). These raw data measurements were then re-formulated for appropriate graphical presentation, data collation, data comparisons, and interpretation. There were, in addition, numerous re-formulations of the furnace construction, particularly of the entry tube bank, for reasons described in detail in the body (Part 1) of this Report.

In the Data Tabulations, Vol. 2 lists the coal analyses and related parameters (1300 entries), Vol. 3 lists the 1-D furnace measurements from 1-91 to 2-92 (6400 entries), Vol. 4 lists the 1-D (generally preliminary) furnace measurements prior to 1-91 ( 1200 entries), Vol. 5 lists the high intensity furnace measurements ( 1100 entries), Vol. 6 lists the 2-color pyrometer data (32000 entries), and Vol. 7 lists additional miscellaneous data (3000 entries). The data were entered into Lotus files and the Tabulations are print-outs of those files. The majority of the 2-color data and all the PSD determinations after mid-1991 were stored in the Lotus files as they were generated. The rest of the data (approximately 5000) were entered by hand. Much of the subsequent data reduction, computations, and final data generation was carried out also in the Lotus files. 
The flame has also been numerically modelled in an associated project. As originally formulated, this present Project was to include the program of flame modelling including the sulfur release; however, preparatory work carried out after approval of this present project but before it was funded indicated that the modelling was likely to be a much larger and more complex task than was originally envisaged and that inclusion of this Task in the Project would probably be adverse to its completion as originally formulated. Fortunately, it was possible to obtain independent funding for this modelling Task from the Ohio Mining and Mineral Resources Research Institute (OMMRRI), and the modelling Task was started in $1987 / 88$. Accordingly, as the modelling started substantially in advance of the rest of the Project, this by agreement became a shell Task in this project, for reporting as relevant here, and the key results, by agreement, are included in this Report.

The objective of the work as defined in the Work statement was to determine the combustion and sulfur release profiles from relevant coals and to compare these with the sorbent characteristic behavior in association with flame modelling.

Time Table and staffing. The Proposal on which this work was based was drafted in Fall of 1986 (Summary Proposal), with a full proposal submitted on request in spring of 1987 and approved for funding as the project Number shows in mid-year of 1987 . It was not funded, however, until late Fall of 1989 and with this 
timing, in consequence, only one full-time graduate student was available until mid year 1990; in the interim, considerable use was made of part-time, undergraduate students. However, the delay did permit the (unfunded) preliminary work that revealed the scale of the modelling Task, as already noted above.

Principal Results. Overall, the modelling was substantially successful; the experimental program less so in terms of the originally defined targets as the result of unexpected findings of flame behavior, but those same unexpected findings are unknown results, not previously reported and evidently showing previously-unknown stable flame forms that scientifically are of major interest and probable importance though they militated against completion of the Tasks as originally defined.

Flame Modelling. The details of the flame model are given in the body of this Report but, briefly, the model is a one-dimensional, radiation-kinetics formulation for combustion of pyrolyzing coal with a typical particle size distribution (PSD) at entry that is described by Rosin-Rammler; the kinetics includes the factors of pyrolysis, volatiles combustion, boundary layer diffusion to the formed char followed by joint internal and external gas-solid reaction governed by Langmuir kinetics. The predictions include profiles of pyrolysis, burnout, oxygen consumption, temperature, and change in PSD and particle density. This model is an extension of a previous char-only formulation developed in the period 1974 to 1980 that successfully predicted then-available flame profiles. 
In reformulating and extending the model from char to pyrolyzing coal a number of unexpected, mainly-numerical difficulties were encountered, notably an initial problem of convergence. This led to extensive investigation into the internal structure of the model details for resolution of the problems. It was originally anticipated that reformulating and extending the model would take 2 to 4 weeks but, in the event, this took more like 18 months to 2 years. This was one of the results that showed the model development was likely to be a much bigger task than originally expected; it may be noted rere that the final form of the model takes 4 to 6 hours of VAx time for computing one flame (corresponding to about 1 second in real time).

In the process of analysing the internal structure of the flame model it was found that part of the problem was evidently the result of changes in developments of hardware. The original program was written for batch processing on an IBM 360 mainframe for which it was required, when running most iterative programs, to limit iterations to a relatively small number (typically 50); the current program is interactive, written for processing on a VAX 8550, and the number of iterations is virtually unlimited. on the IBM mainframe, where final convergence was very slow on account of the nature of the program, it is now evident that convergence was probably more apparent than real though, in retrospect, the results were often approximately good enough. Nevertheless, the faster machines of the 1980's compared with the $1970^{\prime}$ s may be revealing behavior not evident in the earlier runs. 
In particular, there is some indication that the final flame form (combustion profile) could possibly be influenced or even determined by the temperature profile used as the initial condition to start the iteration; in other words, there could be different "windows" of stable operation. This could have major practical significance as discussed in due course. So far as we have been able to establish, however, the essential predictive results obtained earlier are substantially correct.

At this time we are now able to predict flame profiles, that is variation with distance or time, for temperature, oxygen consumption, reaction efficiency, particle sizes (with the distribution split into 10 or 20 sizes), particle density (also for the different size fractions), and sulfur (as $\mathrm{sO}_{2}$ ). The change in mean particle size (representative of the PSD changes) is also obtained. To the extent that relevant experimental measurements are available, the model predictions are generally supported by the experiments.

Furnace Measurements. The experimental measurements are less complete in terms of consolidated interpretable results since they showed unexpected flame forms departing from the normal one-dimensional structure. In other words, there appears to be two or more stable windows of operation. This finding was completely unexpected.

The furnace design originated in 1956 with a plug-flow (one-dimensional) construction similar to the present furnace but with a cone-shaped top for flame stabilization. The disadvantage 
of that design was the marked change in cross-section at the bottom of the cone that presented major difficulties both in data interpretation and in model formulation. The current: design, an extension of that original, originated in $1961 / 62$ and included the cone for uniform expansion of the formed cloud across the furnace, but with a water-cooled tube-bank at the bottom of the cone to provide a defined entry for the (cold) cloud that aided both model formulation and data interpretation. original measurements and subsequent investigations since 1961 established that the flame profiles across the furnace were essentially flat, as needed for true one-dimensionality. For the most part, measurements were then made primarily on the flame axis, once the transverse uniformity had been established. In the present program, however, the transverse uniformity was found no longer to exist, and the means of restoring that necessary uniformity is still not fully determined.

The probable existence of the different flame type gradually became evident mainly as the result of $\mathrm{so}_{2}$ measurements, mostly through the spring and summer of 1991 . 'ihe problem as described more completely in the body of this Report was that the sulfux flux through the furnace, calculated on the assumption of uniform transverse profiles, was substantially less than that entering, calculated from the fuel analysis. Search for the missing sulfur revealed that the transverse profiles were not uniform. Accordingly, a series of experiments was started to determine the cause of the problem and its solution; the 
investigation also included a very detailed examination and analysis of all previous experiments and records and, particulariy, those original experiments carried out from 1961 through 1965. Some of the causes are now established, but the full solution is still unclear. Nevertheless, the results at this time support the view that there are indeed at least two stable flame configurations.

The new flame type as it currently forms is a twodimensional cone shape with ignition starting essentially at the walls and progressing inwards on the cone (ignition) surface to the furnace center. Consequently, transverse measurements show temperatures (and gas concentrations) that are substantially curved, not flat. The first, and initially thought to be the most-probable cause for this change in behavior was a difference in coal type and/or the coal PSD from the previous experiments. Accordingly, a range of different coals was tested and, in the tests, a total of 14 coals were used representing varied coal types and PSD's. However, as outcome of the relevant experiments, coal type and/or PSD is not now believed to be a factor, or at least not a significant factor. With that essentially eliminated, attention turned to other factors.

The further investigation of the behavior and its probable causes have now identifed three most probable sources of changes. The first is that over a period of years the existing furnace was progressively re-built as components burned-out or needed to be replaced on account of wear and, in the process, apparently "small" changes occurred (such as the sharpness of 
"leaders" on the water-cooled tubes of the stabilizing bank) that were found to be unexpectely critical in affecting the behavior of the dust flow or the flame shape. In particular, one of the changes included increasing the height of the furnace between tube-bank and outlet from $1.3 \mathrm{~m}$ to $1.9 \mathrm{~m}$, to increase the length or period of measurement in the flame, but this increase in height is now strongly believed to be responsible for flame upset by buoyancy at the bottom of the furnace (this had been observed earlier in 1962 in connection with height changes to the conetopped furnace). There were also changes (mostly for simplification) to the design of the entry for the coal cloud at the top of the cone which was found to affect the transverse cloud distribution quite sensitively whereas the cloud dispersion was evidently rather insensitive to injection using the original but rather complex entry design.

The most significant factor so far identified, however, would appear to be the combination of firing rate, stoichiometry, and, particularly, method of firing on light-up. In particular, in a series of experiments at different firing rates and fuel concentrations it was evident that the cone-shaped flame front flattened out substantially as both firing rate and coal concentration were increased, and the flame appeared to converge towards the flat limit required. The key factor, however, may very well be the precise method of firing the furnace for lightup since this determines the initial temperature profile in the furnace. Reasoning, then, from the tentative results of the modelling in which the initial temperature profile possibly sets 
the final stable profile to which the calculations will iterate, we now believe that this is quite possibly matched experimentally by the light-off procedure. Analysis of the past procedures for light-off, to the extent that these were sufficiently well documented, provide some support for this but, to test it would have required construction of a light-off burner of the type used in the past and it was at that point that the project had to be terminated. Whe key point appears to be that if there are (at least) two stable windows of operation then the window the flame stabilizes in is most probably determined by the initial temperature profile on liạht-up and this is determined, in turn, by the actual light-off procedure. It was not possible to test this postulate experimentally for lack of time.

In principle it should be possible to test the hypothesis by computer modelling, but this can not be done with the present model since, first, it must be modified once again to include two-dimensionality. The model then will be so complex that solution on the VAX will probably be impossible; solution on the CRAY should be possible if the flow is still laminar, but not if it is turbulent. In that last case, solution is only expected to be possible on massively parallel machines (the CRAY has insufficient parallel capability).

conclusions. The principal conclusions are still positive. The modelling work established that detailed predictions for the one-dimensional flame can be made, with the predictions generally in agreement with experiment to the extent 
that this could be tested against the limited (One-dimensional flame) data available.

The experimental work did solve a number of operational problems of feeding mixtures of coal and sorbent (limestone) into this special type of furnace, and did also show the possiblilty of measuring the sulfur profiles without and with sorbent present so that the potential of determining the differences for testing the sorption model is evident if the operational problem of the flame structure can be resolved. Resolution of that flame structure problem is believed to be close in view of the results obtained as already summarized. Accordingly, it is our view and recommendation that the inital targets could now be reached at relatively little further cost if the project was to be continued. 


\section{PART II: TECLNICAL REPORT}

\section{INTRODUCTION}

In this Report we present the results of a research study aimed at determining the characteristic profiles of sulfur release from different coals in a one-Dimensional pulverized coal flame.

The incentive for this study was the uncertainty in the structure of the sulfur release profile and, consequently, uncertainty in the degree to which the sorption profile history would or would not match the release profile.

The context of the study is self-evident, and arose from the well-known problem of emissions control of sulfur oxides using ohio coals. This problem has been so extensively discussed in recent years that even a brief summary is essentially redundant; it will suffice at this time that this study is aimed ultimately at the behavior and characteristics of sorbent injected into flames, as in the LIMB process. A first objective in that aim is the need to understand the release profile and this, in turn, requires a full understanding of the combustion profile since the rate of sulfur release from the coal is totally controlled by the combustion behavior. 
In typical previous studies to determine the combustion behavior, however, the flame systems used were jet flame furnaces in which as is well known there is almost total indeterminancy of the particle trajectories and, thus, of the particle temperaturetime histories. Consequently, interpretation of the sulfur release and sorption mechanisms was largely guesswork on that account. The unique element of the present study was the availability of the one-dimensional flame furnace that was developed by the Project Proposer and for which we now have a 30year history of operating experience. In this, the particles flow from top-to-bottom in the furnace so that the particle histories are accurately known. This characteristic was the basis for the original proposal. In the event, however, for reasons that are still only partially understood, the flame system was different from what had been observed in the past.

In all earlier experiments the structure of the flame was quite simple, with a flat, horizontal surface and with the particles flowing from top to bottom in the furnace. In the construction used in this furnace, it now appears that apparently "small" differences in design have resulted in a different type of flame structure. This is described in more detail in the text following, but in summary, it appears more as an inverted coneshaped flame front with substantial horizontal variations in flame properties (e.g., temperature and gas concentration). Evidently, there are two possible stable flame configurations in this type of furnace, with the present cone-shaped flames not previously reported. Nevertheless, the flow pattern is still 
"once-tlirough" so that the problems experienced in the jet flame furnaces do not apply. Because of the more complex flow pattern, however, the interpretation of the data is more complex. 


\section{EXPERIMENTAL}

Figure 2.1 illustrates an overall schematic of the expeximental system. This consists, essentially, of the furnace, with air and fuel feeders and controllers, analysers and monitors for gas and temperature measurements, and other ancillary instruments and equipment for particle size analysis (PSA), furnace light-off, and related activities. At the start of this project most of the equipment and instrumentation was in existence; however, a substantial effort was required for bringing new instruments on-line and also for equipment modifications, renovations, testing and calibration. All components of the experimental system, including the method of data compilation and reduction, are described in this section.

\subsection{Furnace}

Figure 2.2 illustrates the plug flow furnace: it is a vertical combustion tunnel of insulating fire brick, standing variously about 2 or 2.5 meters high, of $64 \mathrm{~mm}$ (2.5") wall thickness, and of square cross section, $165 \mathrm{~mm}\left(6.5^{\prime \prime}\right)$ on a side. The tunnel is topped by a cone of square cross section, and the incoming coal dust and air are supplied at the apex of the cone with a water-cooled tube bank at the bottom of the cone, at the entry to the parallel section of the tunnel, to act as a radiation flame stabilizer. Flow of the fuel-air mixture is vertically downward, through the tube bank, and continuing down through the combustion tunnel. A series of ports at successive vertical positions below the tube bank allows intrusive access 
for obtaining gas and solid samples. These also allow nonintrusive access for particle temperature measurements using twocolor pyrometry. The distances below the tube bank for each port are given in Table 2.1. Wall temperature measurements are obtained using Pt/Pt-10\%Rh thermocouples mounted in wells flush with the inside of the furnace wall at successive positions below the water cooled tube bank, at approximately 5, 10 , and $20 \mathrm{~cm}$ intervals.

For easier independent control of firing rate (fuel supply rate) and stochiometry (air rate at fixed firing rate) the total air is split into conveying (primary) air and supplementary (seconaary) air, and the two streams are independently supplied to the top of the cone. The cone then has two functions: it provides for the total mixing of the primary and secondary streams at the apex of the cone; and it provides for uniform expansion of the totally mixed cloud across the full width ( 165 $\mathrm{mm}$ ) of the furnace proper (the parallel-section segment).

The flame-stabilizing tube bank at the connection between the cone bottom and the refractory section consists of two staggered rows of water-cooled tubes, as shown in Fig. 2.3, with sharp-edged triangular leaders to prevent dust deposition; this bank acts as a radiation trap and allows radiative flame holding. In early experiments (in the 1950's), with the absence of the water cooled tube bank, the flame was allowed to stabilize in the cone. In this form, the mechanism of stabilization is velocitybalancing. The change in cross-section from the cone to the parallel section, however, results in discontinuities in the 
vertical gradients of the flame-property profiles (such as gas analyses and reaction efficiency), in addition to creating a system that is geometrically difficult from the analysis and modelling point of view. These factors were the principal drivers for introducing the water-cooled tube bank, to eliminate the slope discontinuities in the property profiles, and to create a cleaner geometrical system for theoretical analysis.

2.2 Material Supplies

2.2.1 Fuel supply. The main components of the fuel supply system shown in fig. 2.3 are a commercial variable-speed spiral-screw conveyor (Vibra screw Inc., type scR-20), a vibrating tray feeder, and a venturi for entrainment of the coal dust into the primary air line. The flow sequence for the coal is as follows: The coal falls from the spiral screw conveyor onto the vibratory tray feeder and from the vibratory tray feeder into a funnel directly over the venturi where the coal is picked up by the primary air. Because the venturi action entrains additional air into the primary air line, the vibrating tray feeder and funnel are enclosed in a plexiglass box which is sealed except for pressure taps and a bleed from the (monitored) primary air line. The bleed is fed into the plexiglass box and adjusted with a control valve so that the pressure in the box is just atmospheric or slightly negative.

Calibration. To calibrate the fuel supply system, before each set of furnace measurements the feeder was disconnected from the primary air line and calibrated for the given coal by measuring supply rate (the quantity by weight of coal dispensed in a 
measured time) at different feed settings (rate of rotation of the feeder spiral). Typically, the calibration curve of feed rate against feed setting is approximately linear.

Modification of original Fuel supply. The standard fuel feed system used prior to these experiments utilized the vibra screw type SCR-20 spiral-screw conveyor which fed coal into the primary air line through a jet-injector pick-up. The coal was then carried in this primary supply line from the pick-up point to the top of the feeder cone. These elements are still the core of the present system modified for these experiments.

During the course of the experiments it was found that the fluctuations from the rotation of the spiral-screw conveyor could be picked up in the particle temperature measurements, and also appeared to be responsible for possibly anomalous particle size distribution (PSD) measurements (although this did not appear to affect the other measurement properties). Although the feed device is an open spiral of wire so that there is deliberately substantial slip between the spiral and coal dust to reduce the "chunking" that occurs with solid spirals, nevertheless, the fluctuation in feed was noticeable. The first modification to reduce the fluctations was to weld several short pieces of heavy wire near the end of the spiral somewhat radially across the diameter of the spiral. This provided such additional disturbance to the coal feed as it left the feed tube that it increased the frequency of the fluctuations and reduced the amplitude. 
The next modification to the fuel feed system was to change the jet-injector pick-up to a venturi pick-up in order to eliminate the fluctuations caused by the jet-injector. In the original construction, the jet-injector fed into a 3/4-in i.d. (primary supply) line that was essentially blocked at the pick-up point except for the 1/8-in. i.d. high-pressure air supply point. With this design, although some coal would deposit under the issuing jet, normally a stiady-state would be established so that the deposited coal had no influence on supply. Periodically, however, some of this coal would disperse and the resulting perturbation could be picked up by the temperature and PSD equipment to generate anomalous results. This upset-potential was eliminated by use of the venturi pick-up.

In addition the diameter of the pick-up line at the bottom of the venturi was reduced to increase the air velocity at the pick-up point to ensure proper dispersion of the coal into the air stream, and to aid maintenance of the coal in suspension in the conveying line to the top of the feeder cone. This had the further advantage of increasing the injection speed of the primary coal-air mixture into the cone to aid mixing in the cone with the secondary air.

For most of the conventional measurements (gas and solids samples) this modification to the feed spiral was adequate for the purpose, but not for the PSD and particle temperature measurements. Accordingly, for further smoothing of the feed, an additional vibrating tray feeder was inserted later between the spiral feeder and the pick-up venturi. The flow sequence for the 
coal is now as follows: The coal falls from the spiral screw conveyor onto the vibratory tray feeder and from the vibratory feeder into a funnel directly over the venturi where the coal is picked up in the primary air stream.

With either the injector or the venturi pick-up, the jet/venturi action can entrain unmetered air into the primary Iine. To prevent this, the vibrating tray feeder and funnel leading into the primary air line were enclosed in a sealed plexiglass box. Pressure taps to monitor the pressure showed substantial pressure difference between inside and outside, and when the feed was directly from the spiral feeder to the injector/venturi, this reduced pressure was sufficient to suck the coal out of the feeder even when it had been switched off. To control this, a bleed from the (monitored) primary air line was fed into the plexiglass box, and adjusted with a control valve to the point that the pressure difference was just slightly negative.

2.2.2 Limestone Supply. In the experiments in which limestone was included, the limestone was fed premixed with the coal. Once the two were premixed, the mixture fed uniformly and without difficulty through the fuel supply system. The key to success of this procedure was found to be good initial mixing. After a number of experiments on this, an effective procedure was found to be mixing the coal and limestone in a rotating drum. To do this, the requisite quantities were weighed out and placed in a $30 \mathrm{gal}$ drum. With the drum lid sealed tight, the drum was 
placed horizontally on rollers and rotated for 2 hours, using a belt drive motor.

The only disadvantage of tnis procedure, though a substantial one, is that the mixture ratio is fixed, and there is also no flexibility in the quantity of mixture available for a given run. on that account, substantial effort was put into alternative on-line mixing methods.

In other experiments carried out 10 years ago an on-line mixing procedure had been successfully used though at much smaller feed rates (mostly at 2 or $3 \mathrm{lb}$ /hour of coal). From those earlier experiments it was known that the principal problem in feeding limestone is its adhesiveness, and low flowability. In those experiments, two separate spiral-wire type of feeders were used to feed coal and other solids directly into a feed venturi. In the present experiments, an additional feeder of the Vibra screw design (at $\$ 4500$ ) was not available and in its place two alternative procedures were attempted.

The first utilized the vibrating feeder, set independently from the coal feed for these tests, with a conical container set directly over and in contact with the vibrating tray feeder. This fed directly into the secondary air line. Model conical containers of various angles were tested to determine the angle at which the Iimestone would best feed. Because of internal adhesion, even for the smallest angle cones, it was found necessary to dry the limestone to prevent it from sticking together and on the walls of the cone. The major problem with this system was that the amplitude and frequency of 
vibration of the container varied as it emptied, resulting in dramatic variations of the feed rate. Attempts to control the vibration and keep it constant as the cone emptied proved unsuccessful.

In tha second method, a system was designed in which the limestone was fed with an auger. The tests showed trat the cunsistency and seif-adhesion of the limstone was such chat the auger would simply drill a hole through it, and the feed stopped ever as the cone was vibrated.

2.2 .3 Air supply. All air was supplied from the university ventral compressed air supply system and delivered to the laboratory at 80 to 90 psig (depending on the university load). The incoming line was split to provide the separate primary and secondary streams. The rates in each stream were controlled with gate valves and measured with standard air rotameters. The flow meters were fitted with pressure gauges at the meter inlet, and the volumetric flow rates recorded from the rotameters were corrected to standard volumetric flow rates using the usual pressure correction wich included the local atmospheric pressure and the static (absolute) pressure in the line.

Both rotameters were check-calibrated using pitot tube traverse measurements in a specially constructed flow tube system equipped with local pressure monitors and temperature sensors; the meters were also compared directly with each other. The direct comparison and pitot tube measurements are given in volume VII: Aditional Data. 
Part way through the series of experiments a drying system was installed in the air lines. This came about as the result of a series of problems during furnace runs over a period of about 2 months in which it became clear that the coal was unexpectedly agglomerating. This was partly due to very high moisture of the supply air with, in one instance, liquid water appearing in the rotameters. On enquiry it was found that the drying system for the university supplied air had been out of action for the previous month and the resulting upsets vitiated a substantial number of experiments. In the expectation based on information from university staff that it might take up to a year for bids and installation of a new drying system, small drying units were installed in the air line just upsteam of the rotatameters in the combustion laboratory (which installation was aided by the university maintenance staff). This whole episode occupied about 10 weeks.

\subsection{Gas Sampling and Analysis}

The main components of the gas sampling and analysis system are a water cooled sampling probe, conditioning units to remove particulate matter and moisture from the gas sample, and CO2, CO, O2, and SO2 gas analyzers. All gas samples were collected from the furnace by intrusive sampling using a water cooled probe inserted through the sampling ports at successive vertical positions in the furnace. The sampling probe used to collect tha gas sampies for most of the measurements is a straight line probe 17 in long and 0.19 in i.d. The samples were drawn through the probe and on-line conditioning units by 
individial pumps just upstream of each gas analyser unit. The CO2 and CO analyzers are each MSA Model 303, the 02 analyzer is an MSA Model 4000, and the SO2 analyzer is a TECO Model 40 . Due to the relatively low flow rates and the length of the lines through the conditioning units there was a transient of several minutes before the gas analysis values stabilized. All gas analysis values were recorded manually and subsequently entered into Lotus files.

The conditioning procedure for the $\mathrm{co2}, \mathrm{co}$, and 02 analyzers started with a water bubbler which simultaneously cooled the gas and removed most of the particulate matter. This was followed by several Drierite Towers to remove the moisture. The gas line was then split into three lines, one for each analyzer, and further cleaned of fine particulate matter using 7 micron filters.

The conditioning unit for the soz analyzer was a specially built and assembled unit to hot-filter and dry the gas. For accurate $\mathrm{SO} 2$ measurements care must be taken to keep the sample gas above the temperature at which the moisture starts to condense when it will combine with so2. As the sample is drawn through the water cooled probe it is partially cooled, but the probe length and sampling rate are such that the gas is still too hot for the water to condense. To maintain the gas temperature above the acid dew point the sample gas goes from the probe through heated lines to an electrically heated box, and then through a heated filter. From the filter the sample gas goes through a drier which is a counter-flow tube and shell assembly. 
The tubes are moisture permeable so that the moisture from the sample gas permeates across the tubes and into the dry gas in the shell. Finally the conditioned gas enters the soz analyzer itself where there is an additional tube and shell drier.

All of the analyzers were calibrated prior to each furnace run using zero and one span gas as specified in the manufacturer's instructions. The analyzers were also periodically checked with the manufacturer supplied calibration curves using a zero gas and three to four span" gases.

\subsection{Solid Sampling and Analysis}

Two solid sample collection methods were used for obtaining solid samples from the flame. The first method used conventional, water-cooled suction probes, and these samples were used mostly for surface area measurements and for proximate analysis. The second method used open channel probes of unusual design, and these samples were used mostly for batch PSD measurements using a MicroTrac II L\&N analyser.

2.4.1 Conventional Collection Method. The probe used for most of the solid samples collection is a straight water-cooled probe 50 in long and 0.21 in $i . d$. expanding to a 2 -in filter section at the suction end. This probe differs from the gas sampling probe primarily by increased length to allow for adequate cooling of the particles before entering the filter, and slightly larger diameter to prevent clogging. These samples were collected primarily for specific surface area measurements and proximate analysis. All surface area and proximate analysis data were manually recorded and subsequently entered into Lotus files. 
Surface area was measured with a Quantasorb instrument from Quantachrome corporation in which an adsorbate gas from a flowing mixture of adsorbate and inert carrier gas is adsorbed and then desorbed from the sample surface. The adsorption and desorption processes are monitored by measuring the change in thermal conductivity of the flowing gas mixture. For these measurements, nitrogen was adsorbed by the sample from a stream of $10 \%$ nitrogen in a helium carrier at liquid nitrogen temperature, and then desorbed at room temperature. For calibration, a certain volume of pure nitrogen gas is injected into the gas stream line to match the desorption signal from the sample. This procedure is repeated using gas streams of $20 \%$ and $30 \%$ nitrogen.

To determine the sample surface area, the weight of adsorbate adsorbed at a coverage of a monolayer of nitrogen molecules, $x_{m}$, is calculated using the slope and intercept of the Iinear B.E.T. equation:

$1 /\left[x\left(P_{0} / p-1\right)\right]=(C-1) /\left(x_{m} c\right) * P / P_{o}+1 /\left(x_{m} c\right)$

where

$$
\begin{aligned}
\mathrm{P}= & \text { partial pressure of adsorbate, } \\
\mathrm{P}_{\mathrm{O}}= & \text { saturated vapor pressure of adsorbate, } \\
\mathrm{C}= & \text { constant which is a function of the adsorbate heats } \\
& \text { of condensation and adsorption, } \\
\mathrm{X}= & \text { weight of adsorbate adsorbed at relative pressure } \\
& \mathrm{P} / \mathrm{P}_{0} \text { (calculated from desorption and calibration } \\
& \text { readings). }
\end{aligned}
$$


The specific surface area of the sample is finally calculated by dividing the total surface area, determined from $x_{m}$ (the weight of adsorbate adsorbed at a coverage of a monolayer of nitrogen), by the sample weight.

Proximate analyses were determined using the standard ASTM methods for volatile and ash analyses. The standard VM test is to measure the weight loss after heating the sample in the $950 \mathrm{C}$ zone of a vertical electric tube furnace for seven minutes. The standard ashing test is to measure the weight of the residue after pyrolysis determination by heating the sample in the standard ashing oven as follows: heat the sample to $500 \mathrm{c}$ by the end of the first hour, $700 \mathrm{C}$ by the end of the second hour, and leave the temperature at 700 to 750 C for an additional two hours.

2.4.2 Open Tray Collection Method. An alternative method of sample collection was developed using sedimentation of the sample on a narrow open tray, or slot probe, set across the full width of the furnace. For reasons which are discussed in sec. 5.3, the probe was used primarily to obtain samples for PSD measurements using the Microtrac II particle size analyzer. The tray is water-cooled and also contains water to prevent continued reaction after sedimentation. The samples collected are dispersed in water with a trace of wetting agent for analysis in the MicroTrac II so that collection of the sample in the waterfilled tray is of no consequence. 
Particle size Distribution was measured using the Microtrac II particle size analyzer, a laser-based batch sizing analyzer in which the sample is dispersed in appropriate liquid (usually water) and circulated through a transparent cell. In the cell, the flowing stream is illuminated by a collimated laser beam, and the forward (Fraunhofer) scattered light is collected on a discshaped array of detectors. The intensity of the scattered light and the angle at which it is scattered are dependent on the particle size distribution, and the PSD is calculated using theory of Fraunhofer scattering incorporated in the operating software. The analyzer reports percentage by volume for successive size ranges of particles from 0.7 to 700 microns.

Two procedures were used for handling the particle size distribution data. Originally, the data output was provided on paper from the Microtrac analyser printer, and the results were then entered by hand into Lotus files. Later, a software package and communication cables were obtained to connect a PC to the analyser's computer which allowed the data to be recorded directly on a floppy disc. These data files were then imported and converted to Lotus files.

\subsection{Temperature and Velocity Measurements}

Temperature and velocity measurements of the flame are both crucial measurements: the particle temperature is one of the key parameters for determining the combustion kinetics, and the flame velocity is needed to translate distance through the flame into time since time-based profiles are the only basis on which kinetic determinations can be made. In most cases, the flame 
velocity is calculated by correcting the inlet velocity (from the cold mass flow rate) using the change in density (from the measured flame temperature profile) at each successive vertical position in the flame. While flame velocity measurements are usually fairly difficult, some cross-check in these experiments was possible using direct measurements of velocity with the nonintrusive PCSV laser system (which simultaneously provides inflame PSD measurements). Flame temperature measurements are also difficult to make and are periodically found to be in error due to drift of calibration parameters. On that account, flame temperatures are correlated wherever possible against wall temperatures (though these have their own error sources).

2.5.1 Particle Temperature. Particle temperatures were measured at successive vertical positions in the furnace using a two color pyrometer shown in fig. 2.5. The principle of operation is that the ratio of radiation intensities at two specified wavelengths is dependent on the temperature and emissivity of the radiating particles. Visible radiation from the particles enters a sensor probe consisting of a quartz rod of $6 \mathrm{~mm}$ diameter contained in a water-cooled jacket. The light signal travels down the quartz rod into an optic fiber bundle which is divided part way along into two separate channels each with a different wavelength filter at the end. Each filter has a $25 \mathrm{~nm}$ bandwidth, and the specified wavelengths are 800 and 1000 nm. These particular wavelengths were chosen for several reasons: there is little or no gas phase emission from the flame in these wavelength regions; these wavelengths are far enough 
from the $950 \mathrm{~nm}$ absorption band of the quartz rod; and any nonblackbody behavior of the particles is minimized because the two wavelengths are relatively close. After passage through the filters the signals are converted to analog voltage signals using photo diodes; and after amplification and $A / D$ conversion by passage through a data acquisition system, the digitized $\mathrm{mv}$ signals are stored on floppy discs, then converted to Lotus files for temperature computation. The data from each file was converted to temperatures in the Lotus files using calibration data obtained independently for the 2-color system.

The calibration data were obtained by measuring the approximate black body radiation from an enclosure which was heated inside a commercial gas-fired heat treatment furnace. As this furnace was quite old it was first necessary to refurbish it by replacing the refractory lining and firing ports. The enclosure was built of refractory brick and known from previous tests to be a good approximation to a black body. The radiation from the cubical enclosure was viewed by the pyrometer through a small port in one face of the enclosure, and the (black body) temperature was measured using a $\mathrm{Pt} / \mathrm{Pt}-10 \% \mathrm{Rh}$ thermocouple flush with the inside wall of the enclosure opposite the viewing aperture.

The 2 color pyrometer was also tested for frequency response since the observed pulverized coal flames produced frequent fluctuations of the output signals (this is likely due to a variation of particle temperature with size). To test the pyrometer frequency response, a chopper was placed between the 
probe and a tungsten halogen lamp light source. The two output signals from the 2-color were displayed on an oscilloscope as the speed of the chopper was varied. The results from this test showed that the frequency response of the 2 -color is good to at least $170 \mathrm{~Hz}$.

2.5.2 Wall Temperature. These were measured using TC's in TC wells mounted so the closed ends were flush with the inside wall of the furnace at stations approximately 5, 10 , and $20 \mathrm{~cm}$ apart. The millivolt output readings from the thermocouples were originally collected and printed out on an L\&N multipoint recorder. During the progress of this work, the recorder was replaced by the data acquisition system described in sec. 2.5.3, which converted the $\mathrm{mV}$ readings to temperatures in degrees Celsius, and continuously displayed the output on a PC monitor screen. The software package for the data acquisition system also had provision for transmitting any given data set on the screen display for storage on a floppy disk.

Wall TC's are open to error from a number of sources, the most common being material movement by thermal expansion so that the TC bead is no longer touching the inside tip of the TC well. To overcome this, the TC's are lightly spring loaded to maintain their positions. A less common but more serious error results from ash or slag deposition on the inside wall of the furnace so that the tip of the TC well is no longer flush with the effective inside wall. The TC gradient through even a few $\mathrm{mm}$ of ash/slag is severe and can mean a difference of 100 degrees or more. A 
third common problem is wire breakage in which case the TC has to be removed and rewelded.

2.5.3 Data Acquisition. In the course of the experiments it became evident that the data base that was being generated was becoming too large to be able conveniently to record and collate the data values. Accordingly, a data acquisition system was assembled to collect and record wall temperatures and also particle temperatures from the two-color pyrometer. The system is comprised of Analog Devices hardware including signal conditioners and an $A / D$ board installed in a PC which is controlled by Data sentry software written specifically for data acquisition using the Analog Devices hardware. This is a 15 channel system accomodating 13 thermocouples and 2 two-color channels. According to Analog Devices specifications the hardware can be used for collection rates of up to about $2 \mathrm{kHz}$ for one or two channels. However, the upper limit for the data collection rate depends on the hardware, including the computer, and the software of the data acquisition system; in the present system the collection rate is limited primarily by the speed of the PC. Wall temperature measurements were usually required only every 10 to 30 minutes. Two-color measurements required data collection at significantly higher rates, and these were collected at the fastest rate possible with the system which was about $30 \mathrm{~Hz}$.

Hardware. The Analog Devices hardware consists of a backplane, signal conditioners, and an $A / D$ board installed in the $P C$. Each raw signal (from the wall TC's or the 2-color) goes to a set of 
input screw terminals on the backplane, where it is accepted by a signal conditioner plugged into the backplane. A $24 \mathrm{~V}$ DC power supply is mounted on the backplane and is bussed to provide power to all the signal conditioners. The signal conditioners are each for a single input channel and provide input protection, amplification, and filtering of the raw signal. The output from a signal conditioner is a 0 - loV DC signal compatible with the $A / D$ converter which converts the signal to the digital form required by the microprocessor.

Each of the signal conditioners can be "programmed" to accept input signals within any desired range. Resistors which determine the zero and span of the desired input range are installed in a ranging card which plugs into the signal conditioner. The signal conditioners for the wall TC's are programmable, isolated TC input modules (3B37). Three of the signal conditioners were programmed for mV input signals corresponding to 500-1000 C; these were for the first three wall TC's below the water cooled tube bank. Seven of the signal conditioners were programmed for $\mathrm{mV}$ input signals corresponding to 1000-1500 C; these were for the next seven TC's. An additional three signal conditioners were programmed for $\mathrm{mV}$ input signals corresponding to 500-1500 $\mathrm{C}$; and these were for the last three wall TC's. The signal conditioners for the 2-color are programmable, isolated wideband volt input modules (3B41). Both of the 2-color signal conditioners were programmed for input signals of $0-16 \mathrm{~V}$. 
calibration. After the hardware was assembled, the data acquisition system was calibrated using a DC millivolt power supply. The software package allowed a linear calibration, so that a lower and upper temperature value were entered into the software while the power supply was used to apply the corresponding $\mathrm{mV}$ input for a type $s$ thermocouple at the input terminals.

Software. The software of the data acquisition system allows continuous on-line display of the wall temperatures and the voltage output from the 2-color in tabulated or graphical form, and also periodic transmittal of the data for storage on a floppy disc. The data collection and rate of collection are set (within limits) by the user. The data files created by the software include the date, clock time, elapsed time (if the elapsed timer is set), and each channel reading. Since the data collection rate must be the same for all of the channels and since much higher collection rates are required for the 2-color, the 2-color measurements were collested separately from the wall temperature measurements. The data files created by the software could then be imported and converted to Lotus files.

2.5.4 Particle Velocity and Size Analysis. on-line measurements of particle velocity and PSD were made using the laser-based PCSV (Particle Counter size Velocimeter, from INSITEC Corp.). In this instrument, a focussed laser beam traverses the flame, and sensors measure the intensity of light in the emergent beam that is forward-scattered in a direction slightly off-set from the primary beam. Using Mie theory, the amplitude of the 
scattered signal can then be used to calculate the size of the particle at the beam focus, and the pulse width of the scattered signal is used to calculate the particle velocity. Two beams are used individually, a large beam with a 200 micron waist, and a small beam with a 20 micron waist, for increased range in the size measurements.

Although there are many particles encountered by the beam as it traverses the flame, only the particle in the sample volume (or the volume where the beam is focussed, which is approximately $.01 \mathrm{cu} . \mathrm{mm}$ for the large beam and $.00001 \mathrm{cu}$. $\mathrm{mm}$ for the small beam) provides significant contribution to the monitored signal. The focus volume is small enough that effectively only one particle is in the volume at any moment during measurement. Nevertheless, the final PSD values reported require measurements on substantially large numbers of particles (in excess of 10000) so that one PSD measurement may require up to 5 minutes. The related software transforms the signal readings into file data of particle concentration, velocity, and size distribution.

2 . 6 Data Compilation and Reduction Except for the wall temperature and 2-color measurements, nearly all of the data was necessarily compiled and entered by hand. This was due to the nature of the measurements which required a staggering number of manual operations and therefore manual records. Manual operations and records included: collection of solid and gas samples; weight loss measurements for proximate analyses; injection of adsorbate gas for surface area measurements; sample dispersion in water for PSD measurements, gas concentration 
readings; and surface area analyzer readings, just to name a few. These manual records were entered into Lotus files, while the data collected using the data acquistion system was automatically imported into the Lotus files.

\subsection{Coal and Limestone sources}

2.7.1 coals. The primary coal used in these experiments is an Ohio coal (Powhatan) provided by courtesy of the consolidation coal co. In addition, however, as it became clear that the flame structure was different from what had been obtained in the past, as outlined in the Executive summary (see also sec. 3), a number of other coal sampies were used, to check the extent to which coal type could be responsible for the results observed. It became necessary in fact to use coals other than those supplied by Consolidation Coal Co. after July of 1991 and during the rest of the year, when they were installing a new milling system. The coal supplies used in many of these experiments were samples left from previous work in the combustion Laboratory with additional supplies provided by courtesy of American Electric Power.

All aralyses are given in Volume II of this Report. Coals 1 through 10 and the VT series were from supplies used in previous work, and coals 11 through 13 were donated by the Consolidation coal company.

2.7.2 Limestone. The limestone used was a standard-ground limestone, donated by Essroc Aggregates of Hillsville, PA. The analysis is as follows. 
Analysis of Standard Ground Limestone: Product \#910

Chemical Analysis Minimum

$\begin{array}{lr}\text { Calcium Carbonate } & 87 \% \\ \text { Calcium Oxide } & 49 \% \\ \text { Magnesium Carbonate } & 0.5 \% \\ \text { Magnesium Oxide } & 0.3 \% \\ \text { Calcium } & 34 \% \\ \text { Magnesium } & 0.5 \%\end{array}$

Screen size

$100 \%$ thru 8 mesh

$95 \%$ thru 20 mesh

$60 \%$ thru 60 mesh

50 of thru 100 mesh

Calcium Carbonate Equivalent Total Neutralizing Power in terms of Calcium Carbonate Maximum Mcisture 
Table 2.1: Vertical Distance Below Tube Bank for Sampling Ports

Distance Below Tube Bank (cm)

\begin{tabular}{lccc} 
Port \# & $\begin{array}{l}\text { Prior to } \\
7-31-91\end{array}$ & $\begin{array}{l}\text { Run } \\
7-31-91\end{array}$ & $\begin{array}{l}\text { After } \\
\end{array}$ \\
\hdashline 1 & 12 & 5.7 & $6.41-91$ \\
2 & 24 & 10.8 & 14.0 \\
3 & 35 & 16.5 & 21.6 \\
4 & 47 & 21.0 & 31.1 \\
5 & 60 & 31.1 & 52.5 \\
6 & 70 & 42.5 & 65.4 \\
7 & 81 & 54.0 & 88.3 \\
8 & 93 & 65.4 & 111.8 \\
9 & 103 & 111.8 & 134.6 \\
10 & 116 & 134.6 & 158.1 \\
11 & 126 & 158.1 & \\
12 & 137 & & \\
13 & 149 & & \\
\hline
\end{tabular}




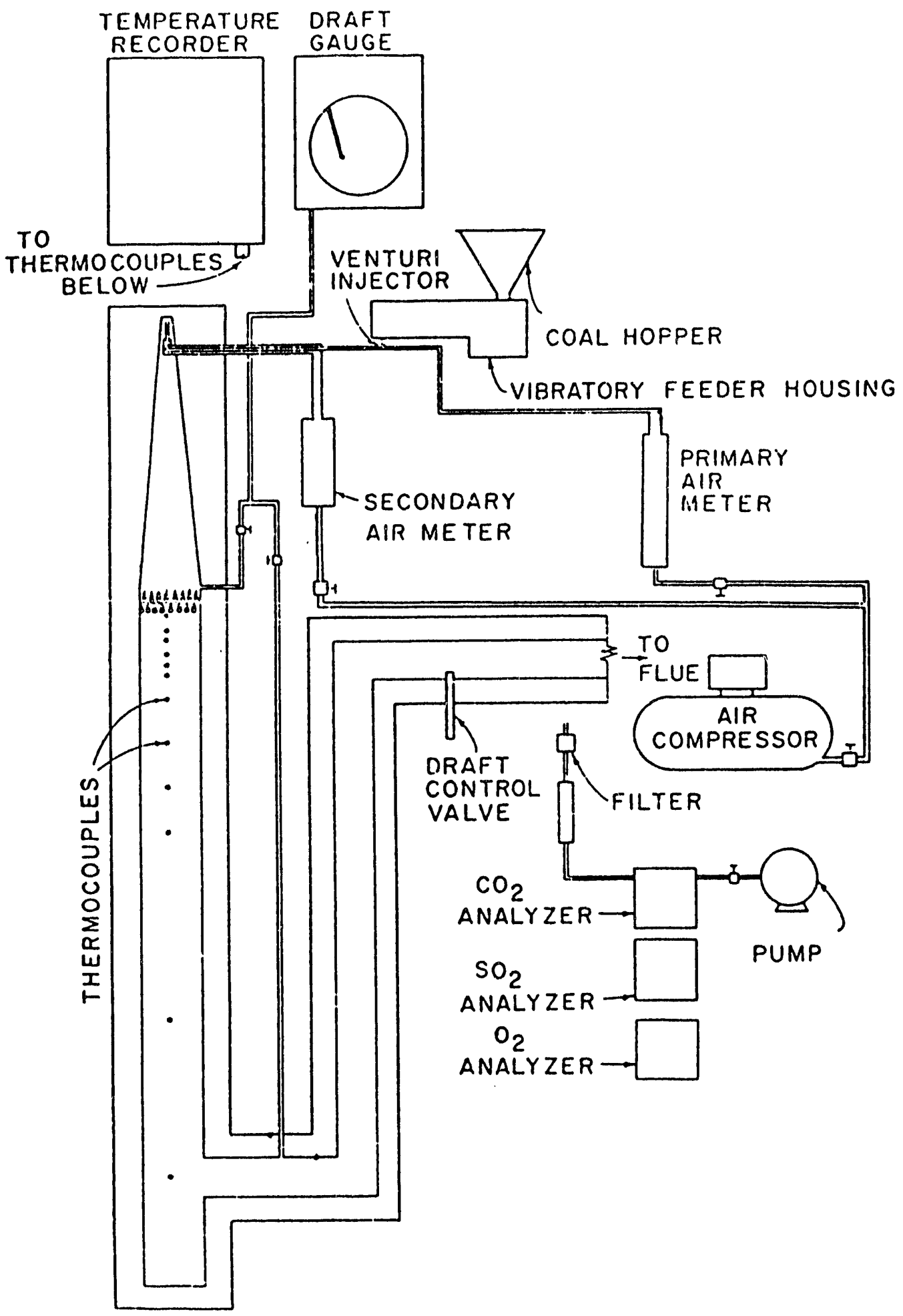

FIG. 2.1 


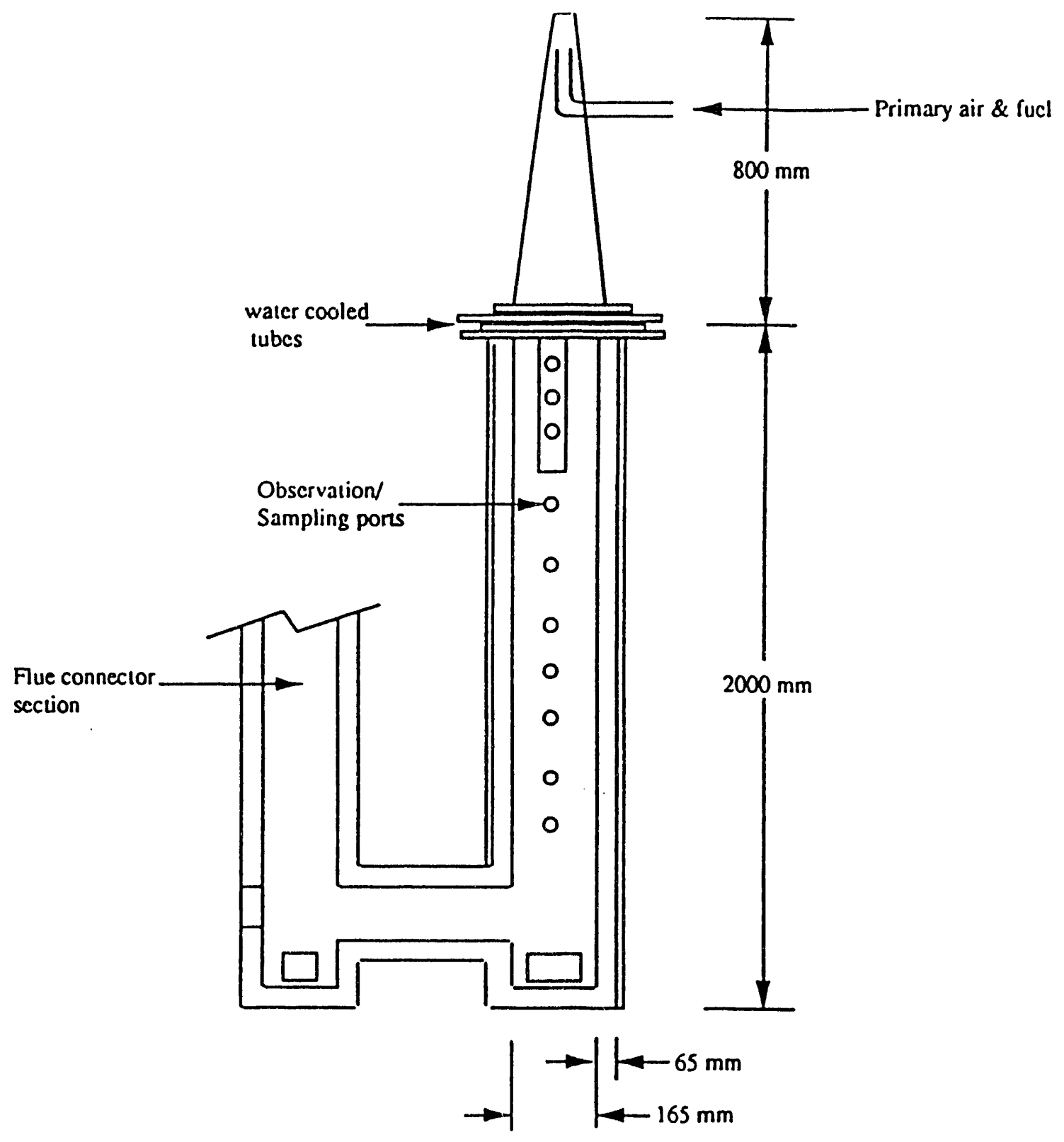

FIG. 2.2 

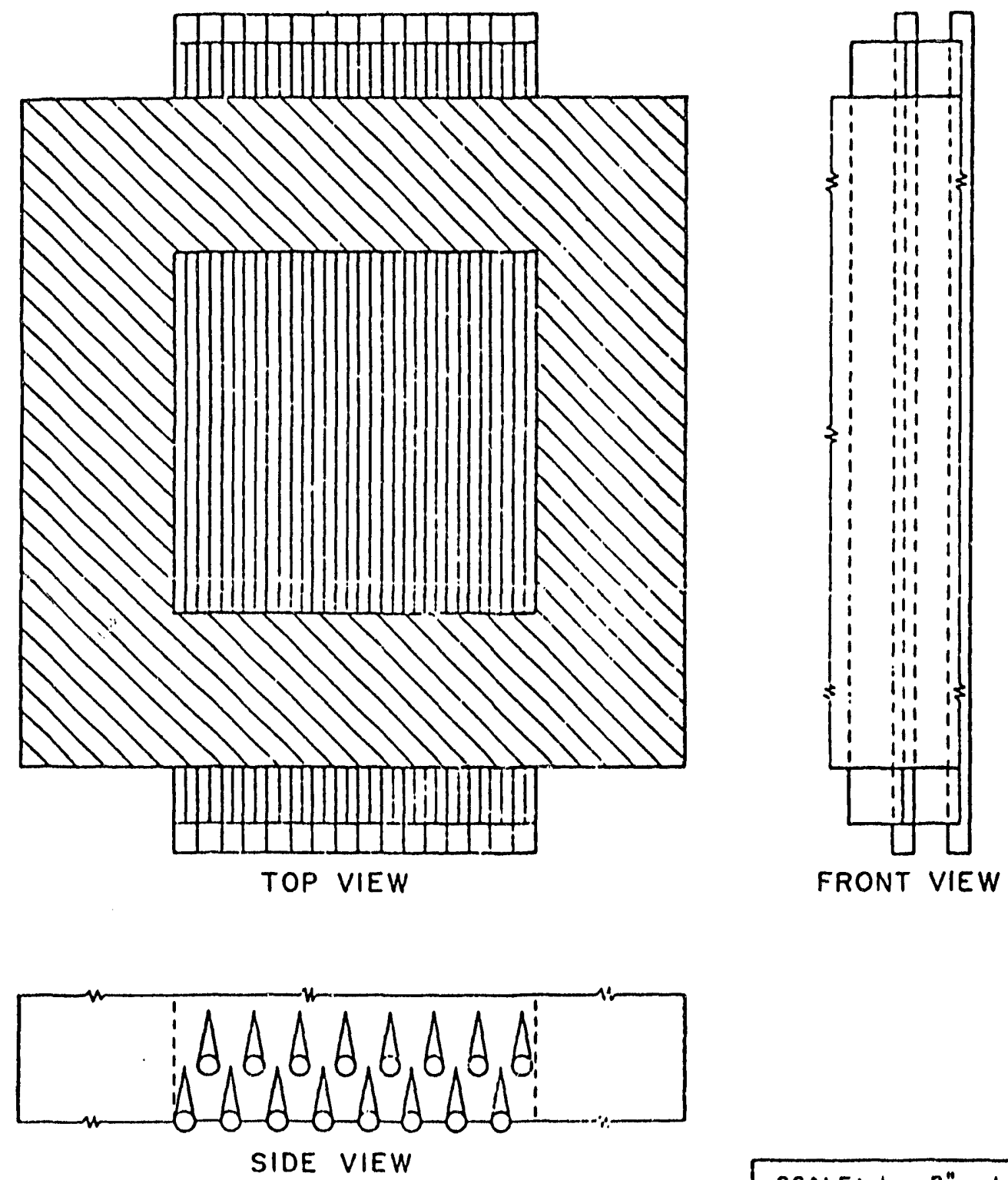

SCALE: $\longmapsto 2^{n}-1$

FIG. 2.3 


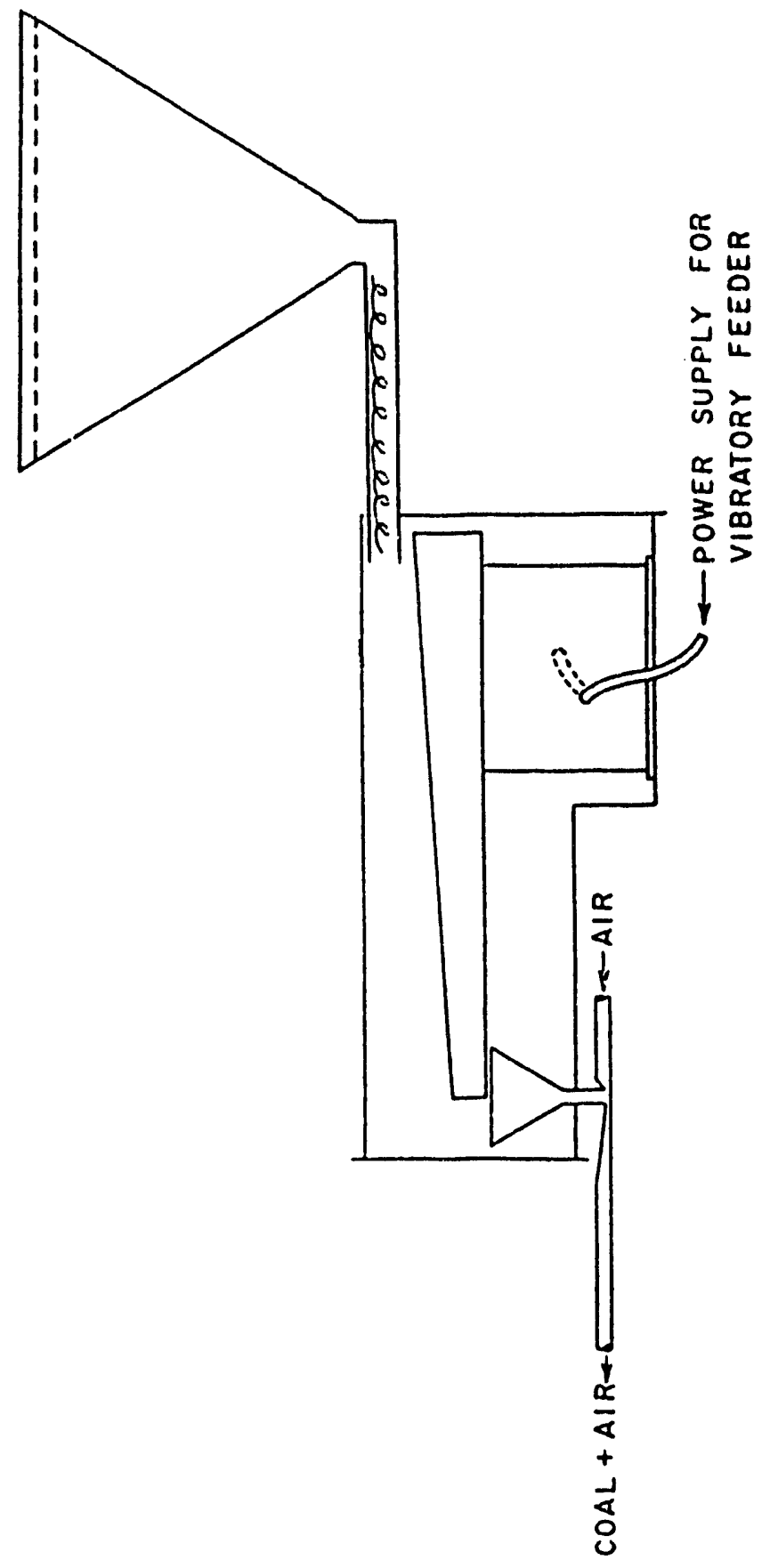

$\nabla$
$\frac{0}{4}$ 


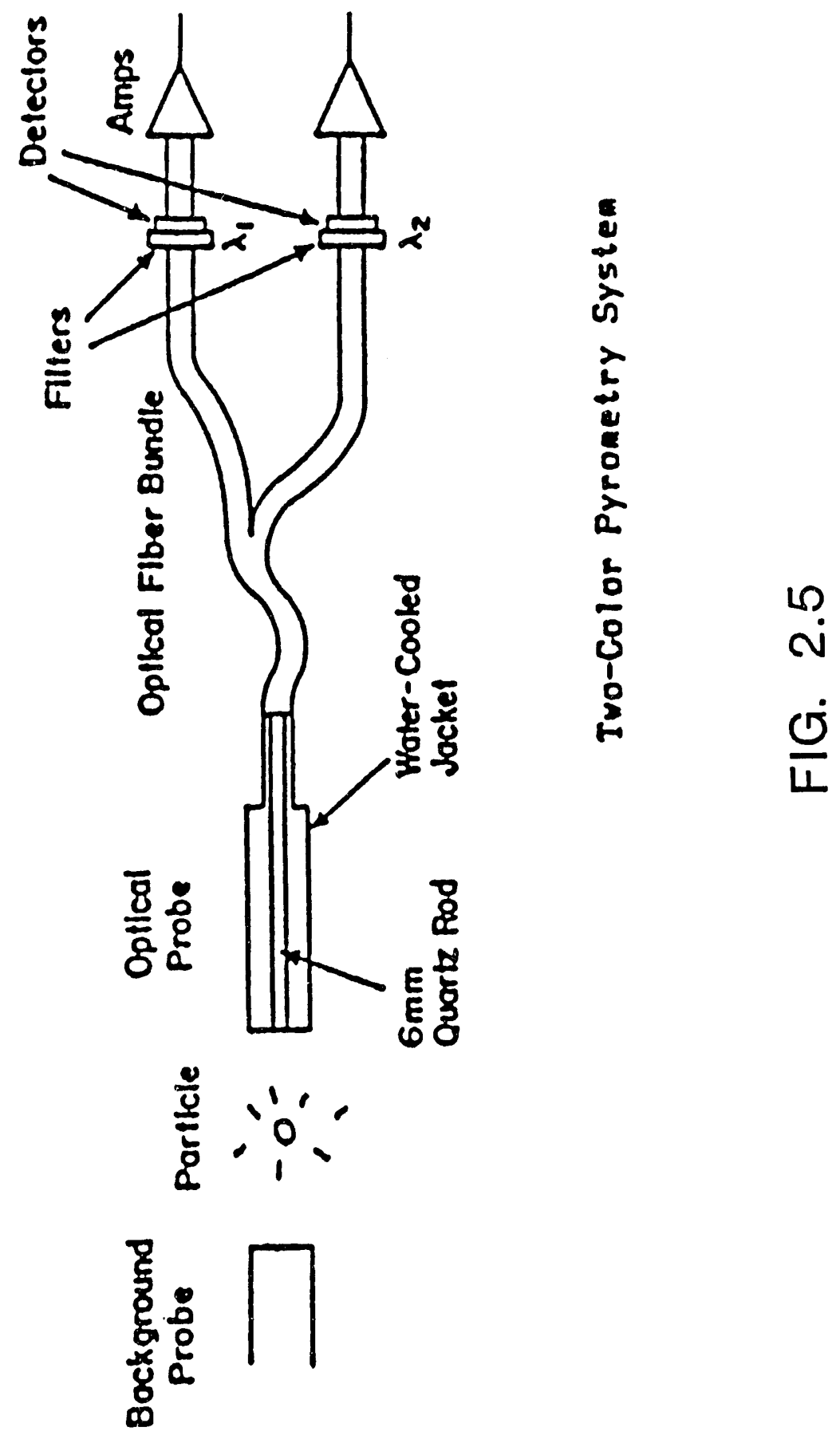




\section{OPERATIONAL PROBLEMS}

In this section we amplify some of the operational problems encountered that are summarized in the Executive summary. The dominant factor is the result described earlier that: It now appears there are at least two stable flame forms that can exist in the furnace used. One is the one-dimensional flame structure that had always been observed in the past; and the second is a cone-shaped, two-dimensional, axially symmetric structure that was evidently obtained in most or all of the experiments reported here. Parenthetically, we note that the only flame system so far modelled is the one-dimensional structure, not the cone-shaped form which is substantially more complex and will require major additional effort to solve.

At this time, however, we still have not been able to identify the conditions that translate one flame form to another although the most likely reason is thought at present to be the method of light-off, as outlined in the Executive summary. This implies that the two flame types are separately stable, and that they do not evidently translate continuously from one form to another. However, this is still undetermined and, in qualitative observations at least there is an evident change in cone angle as firing conditions are changed which carries the associated possibility that, under suitable firing conditions, the cone angle may expand to $2 \pi$, thus converging to one-dimensionality. This convergence has not been directy established; attempts to do so had to be terminated for lack of time. Nevertheless, at this 
time it would appear that convergence of the cone-shaped, twodimensional flames to one-dimensional is only possible, not probable.

The nature and scope of the overall problem of flame type and valid results is most easily understood in the context of "operational windows". In any fuel/air mixture there are well known limits of combustion, the upper and lower flammability limits. These two limits provide initial boundaries of flame stabllity, or an operating "window". In any practical system there are always other additional constraints that essentially reduce the effective size of the operational window, some of which can be fundamental or general constraints, and others that are unique to the particular system. For example, if the coal is too coarse, the propagating flame will not be sustained in any system (though again, this can be jointly a function of the scale of the system); or if the system as constructed cannot handle feed rates above a certain limit, or cannot feed coal that is too fine because it agglomerates, again the window of operation of that particular system is thereby constrained.

As far as possible, the size or controlling parameters of an operating window are determined by investigation, either at the start of any sequence of experiments or during the execution of an experimental program. In some instances, some window parameters or limits cannot be determined ab initio or are unknown, and may only be established by chance during the project execution. For this furnace, the principal limits or "window" of operation were reasonably well known from previous work, but what 
we encountered was a set of circumstances in which, by chance, we found that the system was working near or at the window margin, and this is evidently the source of a number of the problems encountered. In addition, the existence of the two flame forms also represent changes to the envelope of knowledge on the window of operation.

In this section, therefore, we summarize the problems encountered from which we finally concluded that there are, evidently, at least two possible flame forms, with the highest probability at this time that they are independently stable. Establishing this was greatly hindered by the simultaneous but independent occurrence of other problems, some of them essentially transient although we did not know that at the time which further confused the issues. In addition, in at least one case, the same problem (of tube-bank blockage) was due to two different and independent causes, as we describe below. Consequently, definition of a particular problem, determination of its cause, and implementation of its correction were not simple matters and, in addition, the causes were not in all cases fully understood.

The initial three difficulties encountered were: a tendency for the flame to flash back into the supply cone; a tendency to instability even when the flame remained essentially in place below the water-cooled tube bank; and, finally, a tendency for the tube bank to block with deposited coal that bridged over the spaces between the tubes. All these had occurred in the past. What was new was the high frequency of 
occurrence that militated against rapid and accurate data collection.

In the course of investigating these problems, and identifying their sources, additional, larger visual access ports were inserted into the furnace wall just below the tube-bank, closed with plane sheets of high-temperature glass (quartz). With these additional windows, the view of the flame was substantially enlarged and with this view there was then the appearance of a somewhat dished flame with some degree of buoyancy vorticity at the flame front; these views, however, were qualitative and essentially subjective so that they were initially regarded as artefacts of the particular coal and firing conditions. Only later did it appear that they might be representative or indicative of a different flame structure, with the dishing being the top segments of a cone shape. Because the observer is looking through the flame, with variable properties through the flame, interpreting its structure from such qualitative observation is ambiguous at best, and is usually unreliable. Only later, with quantitative measurements did it become evident that they could be symptomatic of newly identified flame structures.

In the course of resolving these problems, the factors considered included: coal type; fineness (PSD); firing rate; stoichiometry; cone entry design; tube bank construction (particularly the tube diameter and spacing); tube bank "leader" shape; primary and secondary air proportioning; primary conveying velocity; pick-up design; regularity/uniformity of coal feed; 
moisture in the coal; moisture in the air lines (primary and secondary): furnace height and draft control. systematic variation of all of these parameters in a standard matrix permutation at two levels (a standard and an alternative value) was impossible as this would have required nearly 500 million different permutations (experiments). Moreover, awareness of the possible/probable influence of each factor became evident at different moments in time during the search for solutions of other evident problems. What now follows is, thus, essentially a summary of the key findings, changes, and conclusions, omitting most of the detailed and of necessity discursive argument and logic on which the actions were based.

[1]. Shape of the "leaders" on the tubes of the tube bank. This was identified as one of the prime sources of blockage. The tubes for the water-cooled tube bank are 3/8-in. o.d., and are surmounted by metal triangular-shaped leaders designed to prevent settling of dust on the tops of the (rounded) tubes. In the original design, as we subsequently established by search of the original records, these leaders were sharp-edged but, in the course of rebuilding the tube bank over several years, the top edges of the replacement leaders had become rounded due to different methods of construction, with a radius of curvature of about $1 \mathrm{~mm}$. After dismantling and rebuilding the tube bank several times with different designs (a two-to-three day task in each case), it was found that the small $1 \mathrm{~mm}$ curvature was, nevertheless, sufficient to provide a base for coal deposits that 
could then bridge across the spaces between the tubes and block the tube bank within an hour or so.

such frequent blockage generally meant that the furnace was fired with correspondingly frequent interruptions, with an associated loss of usable data in spite of the operational time incurred. The frequency of blockage was reduced by sharpening the leaders but this did not eliminate blockage entirely since it was also found to be caused by other factors, and it was not always evident which factors were controlling in any given set of circumstances. Although this was an almost "trivial" design problem, nevertheless, it took an unexpected amount of time to resolve, essentially because it was so apparently trivial. It was certainly no trivial matter to determine the nature of the problem and to resolve it, and it required substantial effort in time and experimental work to do so.

[2]. Moisture. An additional complicating problem overlapping with the leader problem that also resulted in frequent blockage was a period of high moisture in the air lines. In due course, we ascertained that this was due to the failure of the drying machinery used by the campus compressor facility. Ihis finally became evident when water was observed to be flowing through the air-flow meters, and after enquiry about this from the campus Physical Facilities Department. The moisture problem was rectified with the assistance of the Physical Facilities Department by the addition of special drying filter units in the air supply line just before the air flow meters. Prior to that, however, the period during which the problem with blockage was 
becraing evident lasted several weeks during which it became increasingly evident that the leader design was only a part of the problem and that moisture was also a major factor. Distinguishing between the two when neither was clearly evident wis a major complicating factor.

Initially, however, suspicion centered on the coal and, on that account, a number of runs were carried out with different coals to see if this could possibly be a cause. Moisture did vary from one cral to another, but even for the dryest coals, there were still problems with deposits on the tubes, nonetheless, with agglomeration evidently due to moisture. Initially, the source of the moisture was a total mystery and, as noted, the real source was not suspected until the water was observed in the air flow meters. Addition of the drying units eliminated that particular source of operational difficulty, but determining what contribution to blockage was due to each of the two factors of moisture and leader-design was extremely difficult and confusing. At the same time, there was evidence that moisture pick-up by the coal could also be a contributing cause although at this time it is considered minor compared with the other factors.

[3]. Feed uniformity. An adcitional factor that appeared to have some relevance to blockace, but that also had other more serious and pertinent relevance to the flame measurements, was the feed uniformity. With sufficient short-time variation in feed rate, the coal could come through the feed cone in sufficiently strong pulses that the increased local soncentration at entry to the 
tube bank could assist the bridging process. More important from the measurement point of view, the fluctuations could be clearly picked up both by the 2-color pyrometer and during the PSD measurements. For all these reasons it became desirable to improve the uniformity of feed, and this was achieved, as already noted, by insertion of the vibrating tray feeder between the spiral feeder and the pick-up point. In addition, as previously noted, the pick up design was changed from the jet injector system to the venturi pick-up, to eliminate a tendency for deposits to build up under the jet point that would periodically disperse in a pulse.

Subsequent optical measurements showed that the feed uniformity was substantially improved; and it provided some indication that the non-uniformity may have had some influence on the tendency to block.

[4]. Tube Bank Construction. In the course of the re-design and re-construction of the leaders on the tube bank it also became evident that the construction of the tube bank itself required attention, with identified problems related in particular to flash back into the cone. This need for attention became evident during the leader redesign as a result of inspecting the tube bank with lighting underneath. The design of the tube bank, with two staggered rows of $3 / 8$-in $0 . d$. tubes spaced one diameter apart, is such that there is a clear view through the bank from a side angle but, if properly constructed, light in the normal direction is completely blocked. This is required to block the direct radiation normal to the flame front (the less-important 
angled radiation is mostly trapped or absorbed by interceptors other than the incoming coal particles). On inspection, it was again found that small changes in measurements of the tube bank and wall section had allowed the tube spacing to increase to the point that, in conjunction with slight distortion of the tubes themselves, there was then sufficient normal transmission of radiation that flame propagation through the tube bank was frequently possible. This evident problem was essentially resolved by careful reconstruction of the tube bank with more particular attention given to precise diameters, positioning, and spacing.

[5]. Cone design. As part of the concern with, initially, the blockage problem and later with the uniform distribution of coal across the furnace, substantial attention was also given to the design of the supply ports introducing the primary and secondary streams into the top of the cone.

In the original design, the primary line carrying primary air with the coal was coaxial with and inside the secondary air line, with the combined coaxial tubes entering the cone horizontally a few inches below the top, and then turning vertically upwards so that the joint streams impacted on the (truncated) top of the cone. The velocities at this point were very high and, in conjunction with the impact on the top of the cone and the subsequent reversal of the direction of flow, this provided sufficient turbulence for mixing the two streams and for distributing the mixture, apparently uniformly, across the horizontal section of the cone. 
In time the conveying tube wore through by erosion due to the flow of coal particles, and the design was changed several times to avoid problems on that account, and also to allow for easier reconstruction if it did re-occur. These variations included primary, or alternatively secondary feed axially through the top of the cone, with side feed of the secondary or, alternatively, of the primary stream, with or without impact plates opposite the side injection stream to center the jet; alternatively, the two streams were introduced both as side streams but opposed; alternatively, the side stream was introduced through an upward directed jet opposed to the downward directed jet entering through the cone apex. There was also some additional nominal or actual variation in behavior by changing the primary/secondary air ratio as this affected the relative momentum of each jet and thus the relative mixing and jet penetration characteristics.

When it started to become evident that the flame might not be one-dimensional, suspicion centered initially on the possibility that the source of the problem was non-uniform distribution of the coal across the cone cross-section. This proved to be very difficult to determine and it ultimately required the design of the channel or slot probe, with addition of a necessary aperture (port) in the side of the cone for access. Before that probe was available, however, a number of alternative designs for entry of the two supply streams were tried, including a reconstruction of the original co-axial system directed upwards; this also included some variation of the 
primary/secondary air ratio. When these and other changes were found not to have any evident influence on the flame pattern that could be easily discerned, the channel probe was constructed. with it, however, the results showed that although some of the entry supply designs did produce some degree of asymmetry of distribution, the design mostly used evidently did not. Accordingly, at this time it would appear that the coal distribution across the horizontal plane is acceptably uniform. Establishing this result was, nevertheless, a major task.

[6]. Flame Flash Back. The problems described above did reduce the frequency of flash back, but not to the level that was expected. On that account, having re-examined all other sources of potential problems, we re-focussed on the role of the coal type used. This was found to be a significant factor.

The coal selected for use after reconstruction of the cone, the feeder, and the tube bank with new leaders and precision tube spacing, was the ohio coal (\#12 in Volume II), provided by the consolidation coal Co., and it was intended to be the base-line or standard coal for all the experiments. In reviewing the analyses and physical properties of this coal, these parameters all appeared to be within the profile of other coals used in the past, and no untoward behavior was expected. After the reconstructions described, it was, therefore, quite unexpected that the flash back, though less common, should still be occurring at an unacceptable frequency level. By this time, with all other reasonably possible factors examined or eliminated, focus had to be on the coal type. In this instance, 
a careful re-examination and comparison of the PSD using RosinRammler plots indicated that this coal appeared finer at the small fraction end than coals used in the past.

At all events, on the basis of this evaluation, several other runs were then carried out with other coals, and the relative elimination of flash-back with these other coals compared with the ohio coal showed that this coal was somewhat anomalous. At this time, lacking any other clear indications, suspicion focuses on the possibility of higher flammability due to the slightly finer grind as the most probable source of the observed flash-back problem.

This perfectly illustrates the problem of the "window of operation". We did know from work reported to us 25 years ago by investigators using the same furnace design but firing lignite, that the higher flammability of that fuel resulted in frequent flash backs into the cone. Their response which cured their problem was to add a third row of tubes. For bituminous coal, however, all previous experiments had never indicated that the behavior would be likely to cross the boundaries of the operating window. Addition of a third row of tubes was considered in this instance but was temporarily decided against until other factors had been eliminated.

[7]. Buoyancy. Also as a result of re-examining the records of prior work for changes in design, one further change that was then identified was a substantial increase in the height of the furnace between the tube bank and the exhaust port near the bottom that was the connection to the stack. This was originally 
$1.3 \mathrm{~m}$, but had been increased in successive re-builds to $1.9 \mathrm{~m}$, principally to increase the distance or time over which the flame measurements were made. The column of hot gases, however, results in buoyancy forces that, if the column is long or high enough, will tend to cause backflow, up the furnace, against the pressure gradient created by the furnace draft; this can appear as local convection cells. To maintain flow that will overcome this backmix tendency then requires increased draft (increased pressure gradient) which, in turn, can increase air infiltration, amongst other problems; but in particular, during experiments to investigate the behavior, this appeared to be responsible for accelerating the flow in the center of the furnace, thus leading to a tendency for formation of the cone-shaped flame fronts. Reduction of the draft with the longer flame column, however, resulted in appearance of buoyancy-vortex backmix or convection cells just below the tube bank. Accordingly, the top of the furnace was reconstructed to reduce the flame column height to what had been used originally. This appreciation of the probable effect of buoyancy on flame shape came in parallel with quantitative measurements that indicated the possible existence of the cone-shaped flames.

[8]. Flame shape. Focus on flame shape with identification of the cone shapes came about when it became possible to return to investigation of the primary objectives of the project following resolution, particularly, of the tube blockage problem, and it 
was primarily driven by the quantitative measurements of the $\mathrm{SO}_{2}$ profiles.

In accordance with all previous practice, measurements were commonly taken only on the center line since so much previous work had established the one-dimensionality of the flames in past cases. Following the $\mathrm{SO}_{2}$ measurements, however, integratinn of the $\mathrm{SO}_{2}$ concentrations, assumed constant across the furnace diameter, showed that a reasonable sulfur closure was evidently not possible. In searching for the missing sulfur, transverse sulfur measurements were then included in subsequent experiments, and these disclosed the existence of substantial transverse gradients. Following that, the measurements were extended to include the other combustion gases and the temperature. In consequence, determining these transverse gradients at different horizons then led to the conclusion, on a quantitative basis, that the cone-shaped flames existed, and that further vertical profile measurements had no point until the question of the flame structure could be resolved.

[9]. Firing Rate and stoichiometry. In the time and with the resources remaining, it has not been possible to fully determine the source of the different flame structures, and the reasons for the different behavior from that observed in the past. What we have been able to establish at this time is that substantial differences in flame behavior can be obtained by enlarging the envelope of prior operation by substantially altering the firing rate and stoichiometry. In particular, a substantial increase in 
coal concentration but at initially reduced firing rate resulted in a set of results in which the flame was clearly very much brighter, and with wall temperatures up to $100 \mathrm{C}$ higher than in previous experiments, but still with some evidence of buoyancyvorticity at the flame front at a given draft until the furnace height was reduced. When this was repeated at the same draft level in the reduced height furnace, however, it also become evident that the cone angle was increasing (flattening) although it was never possible (at least in the limited time by then available) to pursue this to the point of converging to the original flame form.

[10]. Boundary Layer Influence. In the course of establishing the facts given above, the influence of the boundary layer was also briefly investigated, within the limits of available time, as an additional explanation for the flame shape.

An essential requirement for the flat flame front of the one-dimensional flame is that the velocity profile across the furnace diameter is itself flat, or reasonably so. At the top of the cone, this requirement is reasonably well met by the highlyturbulent flow, where the boundary layer exists but is relatively thin for lack of distance to grow. Although it does grow as the flow expands down the cone, there is a "re-set" condition at the bottom of the cone due to the partial blockage by the tube bank, and the associated split of the flow into multiple streams. Below the tube bank, however, there is no hinderance to boundary layer growth and, accordingly, the possiblility that this was the 
cause of the non-linear horizontal profiles was given as much attention as was possible in the circumstances.

More details are given elsewhere, but the present conclusion is that the boundary layer can be resporisible for some part of the non-linear profiles, but not to the extent found in the experiments.

In summary, the present position is that the conditions under which the two-dimensional, cone-shaped flames and the onedimensional flames are obtained have not been fully determined. It is evidently a matter, in part, of the flame column height (affecting buoyancy), firing rate, and stoichiometry, and possibly in part a matter of coal type and PSD. In establishing these factors, the erratic behavior of the flame due to the tube bank blockage, jointly due to leader design and to failure of the air compresor dryer, were complicating factors that intially completely obscured the flame shape problem. If it had not been for those other, unanticipated, design problems and other elements, it would probably have been possible to resolve the flame shape issue. At this time, however, we are only able to report on the existence of the different flame shapes; the resolution of this problem must be left at this time to future work. 


\section{RESULTS I: THEORY}

The theory presented in this section is concerned with the formulation of the differential equations that describe the one-dimensional flame. The first step in this process is the physical description of the flame as a sequence of physical/chemical processes (the Physical Model) on which is based the mathematical formulation (the Mathematical Model).

As we have described in the Executive summary, the project was formulated on the expectation that the experimental flame system would be one-dimensional, but as work progressed it became clear that there were probably two aquilibrium flame forms: one a flat, one-dimensional form, and the other a coneshaped two-dimensional form. In developing the theoretical systems, however, on account of the timing, only the onedimensional model has been completed at this time, and this is what is presented in what follows.

\subsection{Physical Model}

For translation into mathematical equations (mathematical model) the flame is conceived of as a plane-fronted radiating body infinite in the $y$ and $z$ directions, and propagating in the $-x$ direction against flow of the fuel/air mixture travelling in the $+x$ direction. Since the flame in the experimental furnace is burning vertically upwards, the $+x$ direction is vertically downwards, and the $y$ and $z$ directions are in the horizontal plane. 
The dominant mechanism of flame propagation is heat transferred by radiation in the $-x$ direction (vertically upwards) from the flame into the oncoming cold cloud. The cloud enters the radiation zone at ambient temperature at a defined entry plane, and it heats up as it flows downwards. Experimentally, the defined entry plane is the water-cooled tube bank described in sec. 2. As the clouds heats up in the downward flow it reaches a temperature at which ignition occurs, and the flame is assumed to stabilize at an ignition plane a finite distance downstream from the entry plane. At that plane the flame forms a recognizable "flame front" which is defined for this purpose as the location of onset of reaction where $1 \%$ of reaction has occurred. In the experimental flame system this finite distance can vary, typically, from $5 \mathrm{~cm}$ to $50 \mathrm{~cm}$, or from $0.1 \mathrm{sec}$ to 1 sec, on a time basis, depending on fuel type and/or firing conditions.

As the particles heat up under the influence of the radiation field, after passing through the tube bank, they pyrolyse/ignite, form char, and burn-out in a period of about 1 second. In the process, the sulfur in the coal is released and oxidized to form sulfur oxides (sox). The mathematical description thus requires inclusion of the kinetics for: pyrolysis; VM combustion; and char combustion; and it includes some assumptions regarding the proportioning of the sulfur between the volatiles and the char. At this time, in the absence of better information, it is assumed to be released at a rate proportional to the reaction rate. 
These are all very complex processes, but to obtain solutions using a mathematical model that is sufficiently tractable for numerical solution it is necessary to develop a balance between the total complexity of the actual processes and what is computer tractable. Consequently, idealizations are used that contain what is believed to be the principal or dominant features of the reacting system. At this time the principal processes are described in the following terms.

4.1 .1 pyrolysis. We assume that coal can be represented as consisting of two primary components (Components I and II), where component I forms tar on pyrolysis, and component II first forms a metaplast that then pyrolyses to light gases and char according to the scheme illustrated in Fig. 4.1. This is a scheme that at this time we believe is applicable to all coals, with differences between coals determined primarily by the different quantities of the two components I and II. The magnitudes of these two components then determines the quantity of char; and there is evidence that this is determined by a disproportionation reaction in the Metaplast formation that is also controlled in part by the rate of heating. This is a factor that has yet to be incorporated in the model but at this time the proportioning between component I and component II or Metaplast fraction is set as a constant.

The rates of formation of the different constituents shown in this formulation are governed by the pyrolysis kinetics, but with only two rate constants needed for the three steps indicated. The values for those rate constants are now 
reasonably well known but were only determined to a sufficient level of confidence in the last 5 years. With the Metaplast fraction set as a constant, the rates of formation of tar, light gases, and char are then determinable.

4.1 .2 VM Combustion. Because of the diversity of hydrocarbons produced by pyrolysis, and the changes in composition that can occur by changing the heating rate, modelling of the gas-phase combustion of the volatiles can become a near-impossible task if treated in full. Using a global or operational treatment, however, the combustion rate of the volatiles can be treated adequately by a single rate equation that has been shown to describe behavior of all coals, at least at the level of accuracy that is required for flame modelling purposes.

4.1.3 Char Combustion. Char combustion generally starts after pyrolysis has finished although more detailed analysis shows that it can start before pyrolysis is complete so that there can be some overlap between the two processes. Nevertheless, for purposes of simplicity, this overlap is assumed to be negligible so that the assumption is that: during pyrolysis, the VM efflux screens the particle from oxygen attack but the oxygen can finally reach the particles (and often penetrate them causing internal burning) as the efflux falls to zero. The complete sequence of events then includes: oxygen diffusion from the ambient (interparticle) space to the particle surface, diffusion into the interior of the particle, adsorption on the solid surface of the particle (external or internal), 
desorption of the adsorbed oxygen carrying with it the underlying carbon atom (completing the chemical reaction), and diffusion of the products formed, principally $\mathrm{co}$, back into the mainstream. An effective and computer-tractable equation for this reaction sequence that adequately describes all the chief elements of the process is the Extended Resistance Equation, and it has the form

$$
1 / R_{s}=1 / k_{D} p_{a}+1 / \epsilon k_{a} p_{a}+1 / \epsilon k_{d}
$$

In this formulation, the boundary layer diffusion rate is governed by $k_{D}$; whare internal difrusion occurs, the reaction is enhanced by access to a larger surface area which is governed by the parameter $\epsilon$; the oxygen at the local surface (internal or external) is absorbid on that surface at a rate governed by $k_{a}$; and in due course (a time period that can range from centuries to microseconds, depending on the temperature) the oxygen desorbs, carrying with it a carbon atom (mostly co at flame temperatures).

\subsection{Mathematical Model}

Any mathematical model of a flame requires formulation in terms of the conservation equations for mass, momentum, and energy, in conjunction with the reaction rate and species conservation equations. In these flame systems, however, important simplifications are that the system is one-dimensional and at essentially constant pressure which eliminates need to include the momentum equation. The behavio: of the system is 
then doninated by the Energy Equation in combination with the equations for the kinetics and mass/species conservation.

4.2.1 Energy Lquations: The governing heat transfer equations that we use in the model to describe the energy exchange in a flowing mixture of coal particles and air is primarily a balance between (downstream) convection and radiation, but it includes conduction as well as differences in temperature between the gas and solid particles. We consider in sequence: the Particle energy equation; the Gas energy equation; and Radiation. In the energy equations, the dominant energy flow is due to radiation and convection, but conduction is nevertheless included. The initial formulation is on a distance basis $(x)$.

In the equations following the parameter definitions are as follows: $T$ is temperature; $C$ is specific heat; $m$ is mass flux; subscripts $p$ and $g$ denote particle and gas respectively; subscript $j$ denotes the particle size fraction $j ; q_{s}$ is the heat released due to char combustion; $q_{v m}$ is the gas-phase heat release due to volatile combustion; $h$ is the particle-gas heat transfer coefficient given by the Nusselt Number, with this taken as being equal to 2 (assuming essentially no relative velocity between the particle and the gas); $A$ is the particle surface area; $n_{p}$ the particle number density, and $\eta$ is the reaction efficiency.

4.2.1.1 Particle Energy Equation. The complete suite of particles entering the furnace is divided into a finite set of 
particles of different average size, and each set is followed independently through the flame. Mostly, 10 fractions are used, but in some instances 20 are used. In a thin element of the cloud (of width $\delta x$ ), the following equation describes the local energy balance for all particles in the jth fraction:

$$
\begin{aligned}
d\left[m_{p, j}\left(l-\eta_{j}\right) \cdot c_{p} T_{p, j}\right] / d x & =-\left[d q_{r} / d x\right]_{j}+q_{s, j} \\
& -h_{j} A_{j} n_{p, j}\left(T_{p, j}-T_{g}\right)
\end{aligned}
$$

This represents the balance between change in enthalpy jointly due to net radiation (1st RHS term), due to reaction (2nd RHS term), and conduction from the local ambient (the space between the particles).

4.2.1.2 Gas Energy Equation. This has substantially similar form to Eq. 4.2 but with the inclusion of the term for conduction and lacking the radiation term since the gas is effectively transparent compared with the particles. We have:

$$
\begin{aligned}
-d\left[\lambda d T_{g} / d x\right] / d x & +d\left[\left(m_{g}+m_{p_{1} j} \eta_{j}\right) \cdot c_{g} T_{g}\right] / d x \\
& =q_{v m}+\sum_{j=1}^{M}\left[h_{j} A_{j} n_{p, j}\left(T_{p, j}-T_{g}\right)\right]
\end{aligned}
$$

In this instance, the conduction exchange between the particles and the gas must be summed for all the particles, where the temperature of the different fraction sizes are assumed to be different. 
4.2.1.3 Radiation. The balance for this is based on a net radiation supply to a segment of the particle cloud as the difference between the radiative loss of that segment and the radiative gain from the rest of the cloud including the entry and exit planes.

$$
\begin{aligned}
-\mathrm{dq}_{\mathrm{r}} / \mathrm{dx}=2 \mathrm{kq}_{0,1} \mathrm{E}_{2}(\tau)+2 \mathrm{kq}_{0,2} \mathrm{E}_{2}\left(\tau \tau_{L}-\tau\right) \\
\quad+2 \mathrm{k \sigma}{ }_{0}^{\tau} \int_{0}^{\tau} \mathrm{T}^{4} \mathrm{E}_{1}\left(1 \tau-\tau^{*}() \mathrm{d} \tau^{*}-4 \mathrm{k} \sigma \mathrm{T}^{4}\right.
\end{aligned}
$$

where as defined below, $E()$ is the standard exponential integral governing radiation attenuation between two points at two different locations defined by their optical depths. In the RHS terms: the first is for the entry plane radiation (at ambient, and essentially insignificant), and attenuated to the thin element location at $x$; the second is for the downstream (exit) plane, also attenuated to $x ;$ the third is for the contributions for all segments of the cloud (written for an arbitrarily located segment radiating to the balance element at $x$, and integrated over the whole flame thickness); and the last term is for the radiative loss by the balance element (at location $x$ ) at temperature $\mathrm{T}$ assuming a local absorption/emission coefficient, k.

The relevant definitions for these terms are as follows:

$$
\tau(x)=\int_{0}^{x} k d x, \quad k=\pi r^{2} n_{p, 0}\left(T_{0} / T_{B}\right)
$$




$$
q_{0,1}-\epsilon_{1} o T_{w 1}^{4}, \quad q_{0,2}=\epsilon_{2} o T_{w 2}^{4}
$$

and

$$
E_{n}(x)-\int_{0}^{x} \mu_{n-2} \cdot \exp (-x / \mu) d \mu
$$

4.2.1.4 Ancillary equations. These specify the heat generation terms for the volatiles and char combustion:

The heat generation terms $q_{s}$ and $q_{v m}$ are given by

$$
q_{s}-\left(4 \pi r^{2}\right) \cdot n_{p, 0}\left(T_{o} / T\right) \cdot R_{s} \cdot Q_{c h a r}
$$

where: $n_{p, 0}$ is the particle number density at the entrance plane; $R_{S}$ is the specific reaction rate given by Eq. 4.1; $Q_{\text {char }}$ is the heat of combustion of char; and

$$
q_{v m}=\left[\left(p_{0} / v_{0}\right) \cdot C_{v} C_{o x} m k_{v m}\left(T_{0} / T_{g}\right)^{2}\right] Q_{v i n}
$$

where: $v_{0}$ is the entrance flow velocity; $c_{v}$ and $c_{o x}$ are respectively the mass fractions of volatiles and oxygen; and $Q_{v m}$ is the heat of combustion of the volatiles.

some of the parameters are time dependent as they are controlled by the different reaction rates. For the PSD divided into 10 or 20 discrete sizes, there are, in fact, 10 or 20 such equations, each one describing a different particle size. 
4.2.2 Reaction rate equations. The kinetic processes required are described in qualitative terms in sec. 4.1. Quantitatively, these are described as follows:

4.2.2.1 Volatile Matter Release Equations: The rate equations for the formation of $\operatorname{Tar}(H)$, Metaplast (M) and Light Gases (G) are based on the assumption illustrated in Fig. 4.1 that coal consists of the two primary components, $C_{I}$ and $C_{I I}$. We then can write for the rate equations:

$$
\begin{aligned}
& d H / d t=k_{u} C_{I} \\
& d M / d t=k_{u} C_{I I}-k_{m} M \\
& d G / d t=\nu k_{m} M
\end{aligned}
$$

where $\nu$ is the mass stoichiometric coefficient for light gas formation from metaplast; this is a parameter that is pre-set in the model as used at present, but that is potentially describable in terms of heating rate.

4.2.2.2 VM Combustion: Using the global rate equation, the rate of volatiles combustion is given by

$$
-d C_{v m} / d t-\rho C_{v m} C_{o x} \cdot k_{v m}^{0} \cdot \exp \left(-E_{v m} / R T\right)
$$

where: $C_{v m}$ and $C_{o x}$ are respectively the mass fractions of $V M$ and oxygen, $\rho$ is the density of the mixture, $\mathrm{k}_{\mathrm{vm}}^{\circ}$ is the pre-exponential constant and $E_{V m}$ is the activation energy for $V M$ combustion. 
The values of $\mathrm{k}_{\mathrm{vm}}^{\circ}$ and $\mathrm{E}_{\mathrm{vm}}$ used in the calculations are $1.79 \times 10^{9} \mathrm{~cm}^{3} / \mathrm{g} . \mathrm{s}$ and $17.3 \mathrm{kcal} / \mathrm{mole}$ respectively, using the values obtained recently.

4.2.2.3 Char Combustion Equations: The Extended Resistance Equation (Eq. 4.1) relates the kinetics to the specific reaction rate given by

$$
R_{s}=-\left(1 / 4 \pi r^{2}\right) \cdot(d m / d t)-\cdot(1 / 3) \cdot[3 \sigma \cdot d r / d t+r \cdot d \sigma / d t]
$$

This is an inexact $D E$ for which a reaction (integration) path is required to allow solution. This is obtained from the following density-diameter ( $0 /$ d) relation:

$$
\sigma / \sigma_{0}-\left(d / d_{0}\right)^{\alpha}
$$

where $\alpha$ is related to $\epsilon$ in Eq. 4.1 by: $\epsilon=1+\alpha / 3$. The parameter $\alpha$ is approximately a constant in a given flame system. Most values in flames are in the range 0 to 10 , and more commonly in the range $3 \pm 2$; but it can be as high as $10^{4}$.

Equation 4.10 is then used to convert the inexact DE of Eq. 4.9 into the exact one

$$
\left(\sigma / \sigma_{0}\right)^{1 / \alpha} \cdot \mathrm{d} \sigma / \mathrm{dt}=-(3 \alpha / \alpha+3) \cdot\left(1 / \mathrm{r}_{0}\right) \cdot \mathrm{R}_{\mathrm{s}}
$$


4.3 Solution procedure: The equations sets given above lere solved simultaneously on the ME Department VAX 8550 using a combination of an implicit Totally Backward Finite Difference scheme and a multi-step implicit predictor-corrector method. This was the end result of investigation of a wide number of alternative solution procedures, as summarized below.

In the solution sequence, an initial temperature distribution is assumed, to start the iteration. With that set, the radiative flux and the reaction terms are first calculated, based on the assumed temperature distribution, and this calculation is used to update the particle and the gas temperatures. To determine the radiative flux, the radiation terms are integrated over a finitely thin element and summed over the flame length to obtain the total flux. In performing this integration, the radiative flux is assumed to be constant over the iteration, and it is updated during the subsequent iteration. This creates a new temperature profile, and this new profile is then used as a new starting point. The procedure is continued iteratively until a predetermined convergence level is achieved; various convergence criteria were used, with the most common being changes in the temperature values in sequential iterations less than $0.1 \%$. Using the current solution technique it usually takes from 1 to 3 hours of CPU time to numerically solve the equations.

In developing this program, the current solution algorithm was developed after going through a number of other 
solution methods which were discarded for reasons of either nonconvergence of solution or too slow convergence rates. In the development, the starting point was a program originally written on cards. This was for batch computation on the IBM mainframe available in the 1970's, and the program, accordingly, contained constraints in program design that are no longer necessary or desirable. Notably, the program was constructed to operate with a fixed number of iterations, as required for operation at that time to prevent runaway. Consequently, convergence was often forced by different means, in this instance, by using rather large time steps and by using what was, in effect, an overrelaxation process for the change in radiative heat loss or flux from each flame element. In addition, the downstream boundary was assumed to be a porous black wall when a grey wall is now known to be more appropriate; determining the appropriate downstream boundary condtions was one of the most important and difficult problems. Another intractable problem deriving from the batch operation of the main frames at that time was the knowledge of the exact initial conditions used because these initial-condition cards were changed from run to run, and they were no longer included in the available program deck.

Although the procedure used provided very good agreements with the experimental data available at that time, nevertheless, the detailed predictions needed for the present purposes could not be obtained without using very much smaller steps. When the step size was reduced, however, and some of the other constraints were relaxed, the computations either became unstable or did not 
converge. In time it was established that one of the chief problems in the convergence failure was that the particles and gas temperatures in the original program were assumed, largely on the basis of experiment, to be so close that they could be taken as essentially equal. With this constraint, the particles temperatures were a little low, and detailed calculations finally showed that the upstream radiative flux was just not quite high enough to maintain stable ignition of incoming fresh fuel; consequently, the flame blew-off, but so very slowly that it appeared to be almost stable (i.e., converged). The solution to this problem was to use different temperatures for the particles and the gas with the result that the temperatures diverged quite substantially during the iteration process, but at convergence they were quite close and, indeed, close enough that experimental measurement with typical errors would not have been able to show a significant difference over most of the flame.

Although this procedure ultimately resolved the problem, the changes resulting from having different temperatures also required changes to the solution scheme with the result that a substantial number of different schemes were examined. In the process it was found that a solution scheme that was satisfactory for a char flame or for a Type I volatiles flame was inadequate for the complete coal flame. In brief summary, the scheme used in the original program used a Newton-Raphson method. When this failed with the smaller time steps, the first modification was to change the corrector step based on an elemental heat balance. That was unsuccessful and, in sequence, a series of alternatives 
were tried including: a totally explicit scheme; a solution method based on Newton's method for the solution of non-linear DE's; an implicit multi-step predictor-corrector (Adams-Moulton solution scheme) that had been successful for Type I flames and was, in fact, successful for char flames, but not for complete Type II coal flames. Nevertheless, this ultimately led to a satisfactory procedure.

At this time, the solution method has a basis in an adapted version of Gear's implicit multi-step method for stiff equations, with variants for the integration of the kinetic equations including: explicit methods; or Runge-Kutta; or integration over a finite element (current program method). The latest version of the program uses the Adams-Moulton for the flame equations with a modified Implicit Multi-step PredictorCorrector solution scheme for the coal/char combustion.

4.4 Selected Results. To illustrate the results obtainable from the program, three sets of measurements have been selected to show the degree of agreement with experiment, as part of the process of validation, and these results also identify problems for future solution.

Validation of model programs always requires test against the widest possible set of available results and, particularly, results of others to reduce potential for obtaining apparent agreement by curve-fitting or by introduction of non-general elements to the program. Unfortunately, results available from other sources are extremely scarce and are limited at this time to those obtained in our previous work. The program is arranged 
to provide results in the form of 4 graphs for variation with distance through the flame of: Reaction efficiency; Temperature and oxygen concentration; Particles radius; and Particles density.

4.4.1 Howard Data (1967). Figure 4.2 illustrates the results for a Pittsburgh coal fired somewhat rich. The experimental data are shown, with the prediction curves, in the top two graphs. The experimental data are combined from 3 different but repeating experimental runs (\#'s 9, 11, and 14). The measurements included oxygen and $\mathrm{CO} 2$ concentrations but these are not shown for clarity since they are shown, in effect, in the top left graph after conversion to reaction efficiency. Prediction of the reaction efficiency is clearly very good. The dashed line on this graph is for the local reaction rate showing this peaking as would be expected in the early part of the flame. The experimental temperatures are in two groups. The upper group-set are the flame temperatures measured by suction pyrometer, and the lower set are wall temperatures. The predictions are for the flame temperatures only, and are separated into particle temperatures (solid line) and gas temperatures (dashed line) (the declining dashed Iine is the prediction of the oxygen concentration decay). On the initial rise, the agreement on the temperatures is very good though they are overpredicted somewhat after the peak for reasons that have yet to be identified; this will require further sensitivity studies. 
Significantly, the predicted gas and particle temperatures are almost identical except at the peak where the particle temperatures are somewhat higher over a distance of 10 to $15 \mathrm{~cm}$ (with a flame length of 1 to $2 \mathrm{~m}$ ).

The lower graphs show predictions of changes in particles radius and density. For these calculations the particle distribution is divided into 10 size bins that are then followed through the flame individually, as shown. At this time, no adequate measurements of either radius or density are available, with the exception of some PSD measurements reported in sec. 4.4.3. Consequently, these results must be regarded as predictive but not yet independently validated. Nevertheless, they show expected behavior. Thus, in the graph for radius, the smaller particles are seen to be burning out quite rapidly while the larger paticles hardly change diameter. Likewise, all the particles drop in density essentially simultaneously, reflecting the volumetric pyrolysis assumption, but the effect of internal burning is then substantially different for each particle size, with the smallest particles burning almost volumetrically, whilst the larger particles hardly change in overall density since reaction is confined to their outer layers.

4.4 .2 Current Results. Figure 4.3 illustrates recent results obtained in the current experiments in which the onedimensional model was used for the calculations when the flame was more two-dimensional, with the cone shaped flame front as discussed earlier. The calculations were carried out before the departure from one-dimensionality was fully established, and the 
degree of departure from one-dimensionality is still essentially unknown at this time. Nevertheless, the calculations serve a purpose in showing, jointly, the style and scope of the model predictions with increased number of particle bins (from 10 to 20), and also the relatively close agreement between prediction and experiment in spite of the difference in dimensionality of the flame and the model. Additionally, of course, the onedimensional model is also an approximation to the cone flames, with the degree of approximation improving as the cone angle increases towards hemispherical. consequently, a further valid purpose this comparison serves is to show to what extent the model approximately predicts the actual behavior in spite of the known differentiation, and to what extent, therefore, the experimental flames do or do not depart from one-dimensionality.

The prediction comparisons with experiment are all shown in this instance in the upper right graph, showing oxygen concentration and temperature variations with distance; as before, the two experimental sets of temperature points are for the particles (upper set) and for the wall (lower set). The prediction of oxygen consumption is very good and, if translated to the (top left) reaction efficieny graph -- not shown here -is also good. The temperature predictions are adequate on the rise but are high after ignition (at about $1000 \mathrm{C}$, and roughly where the preditions and experimental values cross). As before, however, the particle and gas temperatures are very close except at the peak. 
The other graphs of radius and density are essentially of the same torm, showing the same pattern as before, and showing no additional features as the result of increasing the number of particle bin sizes from 10 to 20 . This bin number, however, is significant in other respects as shown below; nevertheless, these results overall do show relatively insubstantial departure from agreement taken in the context of the different flame types. Moreover, it is possible that there is other reason for the degree of prediction mismatch that is evident, as discussed below.

4.4 .3 Size Distribution. Figure 4.4 illustrates a different type of plot: the variation of a mean particle size with distance through the flame. These results are for the same run as shown in Fig. 4.3, and in this run, the particles in the flame were batch sampled and batch analysed for PSD with the Microtrac and, for the graphical comparisons between prediciton and experiment, a parameter, $d_{50 \%}$, was calculated. This parameter was the particle size at which the total particle volume lies half above and half below the $d_{50 \%}$ parameter.

The graph shows three predicted lines. The center, solid line, is the model prediction for the half-volume parameter based on the initial PSD for the coal, measured before firing. The two other lines represent two alternative definitions: of mean volume diameter (top line); and volume averaged diameter (bottom line). The general difference between the three definitions is, in essence, the difference in starting point at entry to the flame; 
in addition, the bottom two lines show step-wise changes which are an artefact of the number of size bins, as discussed more fully below.

Otherwise, the three lines and, with a proviso, the experimental results all show, overall, a steady rise in the size parameter as burn-out progresses. This is an essentially common sense conclusion from the radius data shown in Fig. 4.3 which also explains the source of the step-wise characteristics of the lower two lines. Each step represents the moment of burn-out of the smallest remaining particle size. Just prior to that moment, the smallest remaining bin size contains its inventory of particles of almost zero size so that these very small particles still contribute to the total inventory on which the mean volume is calculated, and bias the value downwards. At the moment of burn-out, that smallest bin size is removed from the basis for calculation with the result that the mean size of what is remaining must increase. Immediately after that jump, the overall mean diameter starts to decrease since all particles still in the inventory are shrinking in radius with the result that the mean size declines (negative slope) until the next smallest bin size burns out and is also removed from the inventory.

The predicted and experimental curves now show some clearly defined and understandable trends. In the first place, both the overall rise in mean diameter with burn-off is the result of a rising "center of gravity" of what is left as the smallest particles are progressively burned out. The step-wise 
form of the lower two curves is determined by the number of size bins, and is an artefact of the program that requires finite sized bins. In principle, if the number of size bins is increased indefinitely, the step curves will converge to smooth curves. However, as a matter of practicality, this is not possible because the computation time increases non-linearly with the number of bins, and the number used for this graph, of 20 , was chosen to strike a balance between the computation time and acceptable discontinuity. With an infinte number of bins, the program becomes impossible to run. The program also develops further problems from the largest size bins where, with, sufficient sub-division, the largest bin can contain only a fraction of one particle, which is a meaningless concept in this context.

The experimental results now show some broad agreement with the prediction trends, with some important differences. The initial value of the $d_{50 \%}$ parameter agrees with the prediction of the model. In all cases, the predictions then show constant size parameter until pyrolysis is complete. At that point, the first experimental measurement then shows a sharp drop in mean size, by almost half, and at this time the exact cause is unknown. The most probable reason is shattering of the particles as they enter the flame. Neverthelss, following that, the rise in mean diameter parallels prediction until near the end of the flame at about $1 \mathrm{~m}$ when the experimental result again shows a sharp drop. Here again, the exact reason is unknown, but if true and not an 
experimental error, this is most probably due to fragmentation as particle continuity fails from internal reaction. 


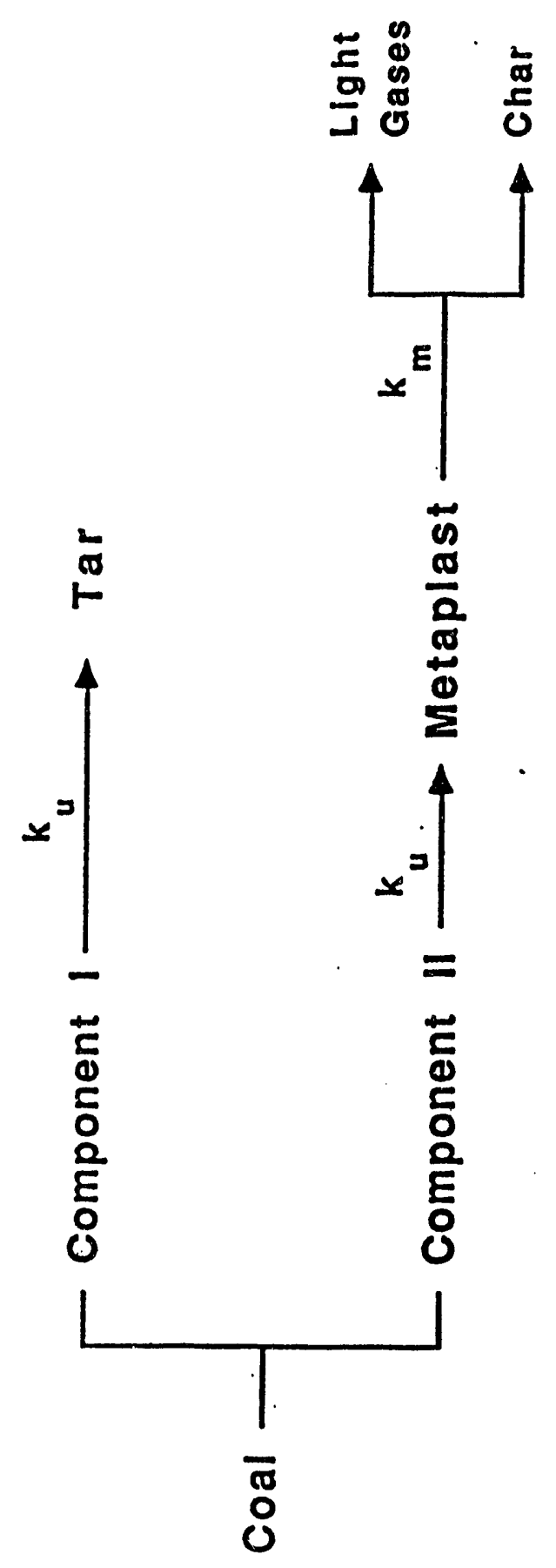

مُ

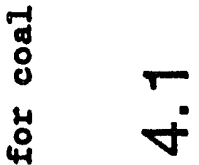

ஜ̊

岕 


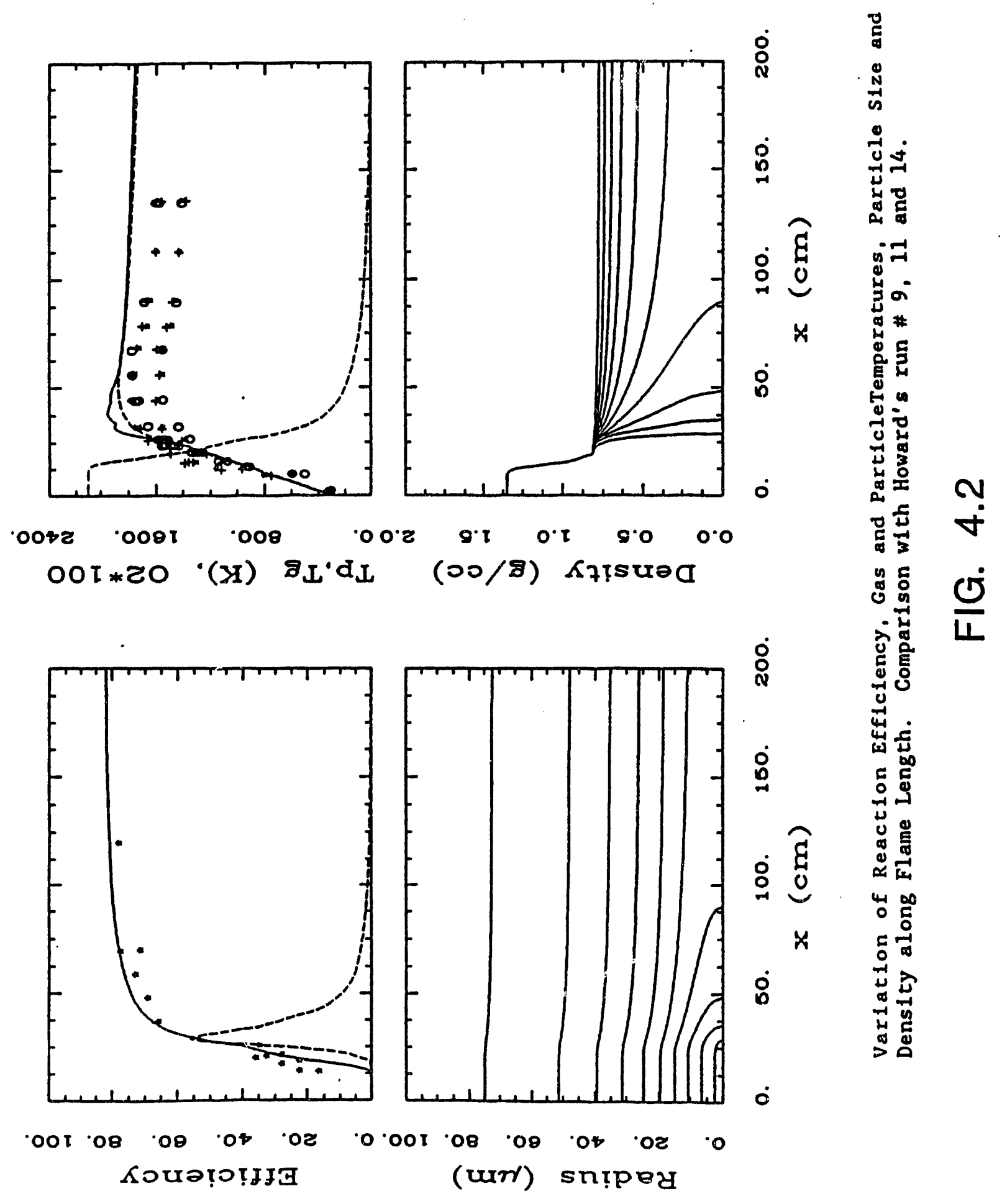




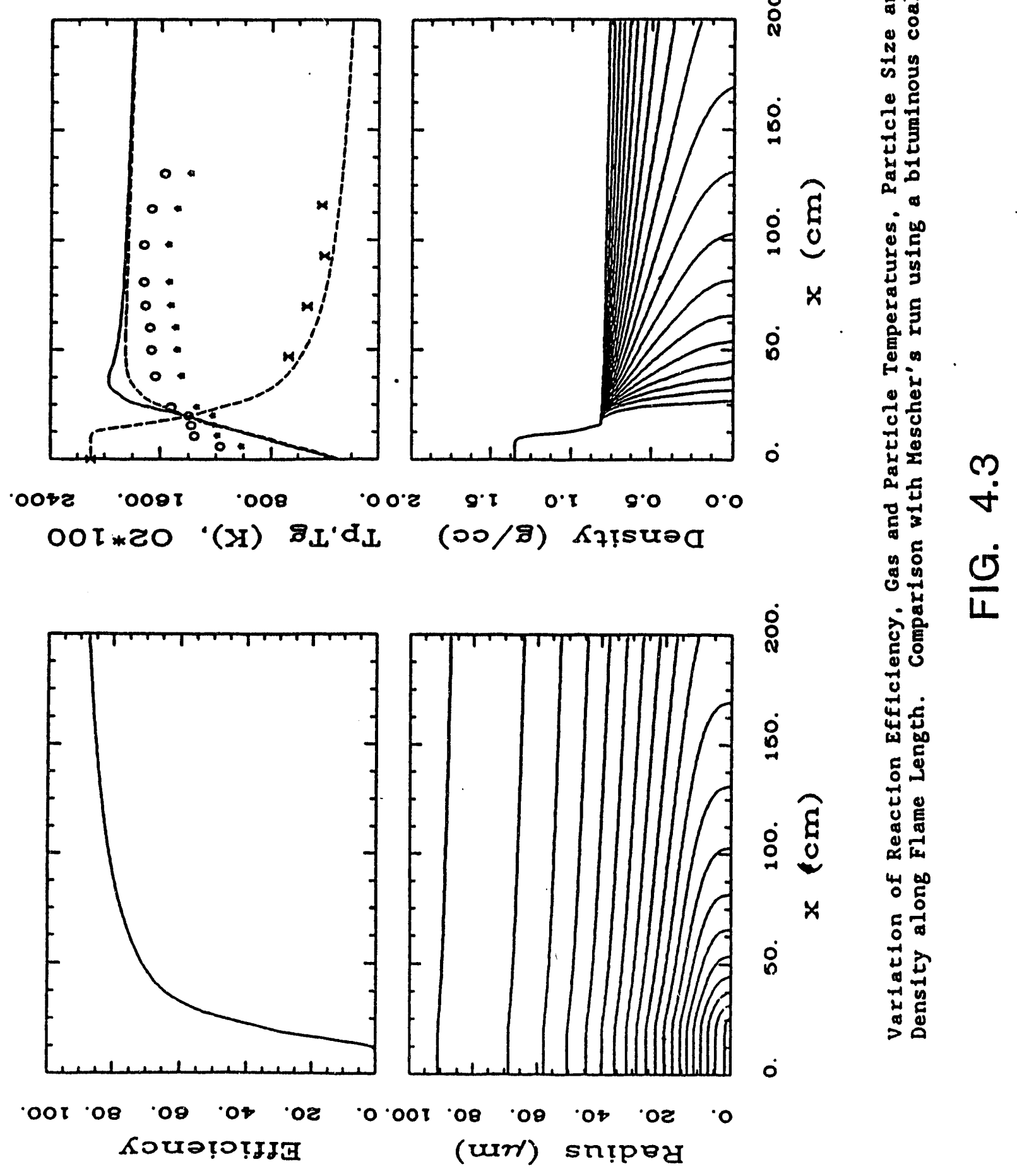




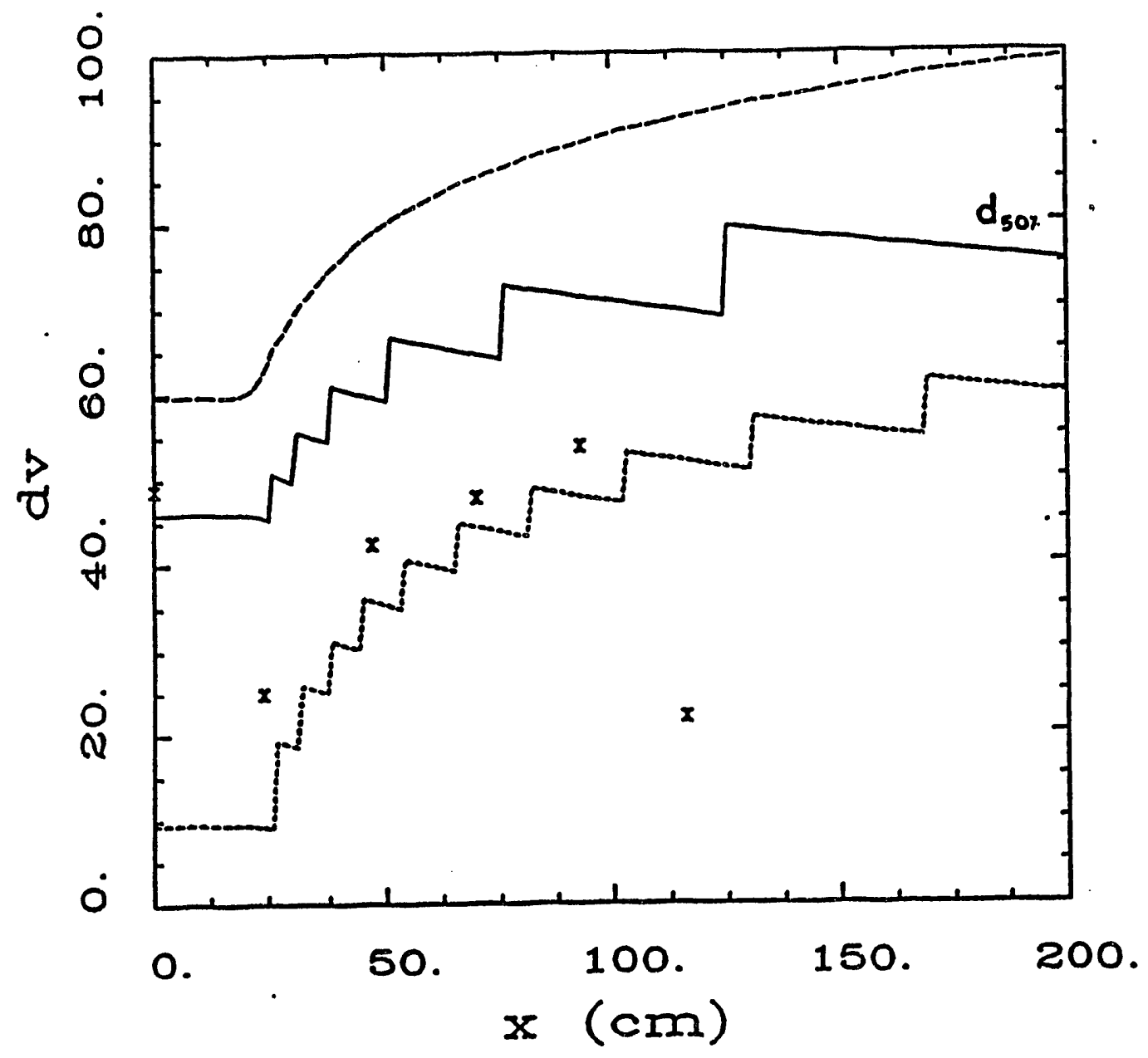

Varlation of mean volume diameter with distance in a one-dimensional flame: Comparisor of prediction with experimental measurements. [" $x$ " measured 508 volume diameter].

FIG. 4.4 


\section{RESULTS II: DEVELOPMENT OF SAMPLING AND ANALYSIS TECHNIOUES}

5. I sampling procedures. Flame sampling is an intrusive procedure in which a suction probe of appropriate design (generally water-cooled) is inserted at the required point in the flame, and a sample of the combustion gas, also containing partburned particles is withdrawn, with the particles either washed out of the stream in a washing bottle at the probe exit, or captured in a filter for subsequent analysis. In this section we are addressing the problems of solid samples captured in a filter and their analysis. Analysis of the solid sample can include proximate analysis (VM, fixed carbon, and ash) and/or particle size distribution (PSD) in batch analysis. Included in this is the associated problem of analysis of mixed coal/limestone samples.

There are several potential problems in this sequence, the most important being the influence of sampling technique and handling methods on the validity of the sample. Techniques for sampling from PC flames and the analysis of the samples have been broadly developed over 4 or 5 decades, but special problems were anticipated in these experiments from sampling and analysis of coal/limestone mixtures. These expectations were supported by the results; but, in addition, it was found that the detailed PSD measurements brought into question some of the conventional solutions to sampling problems, and these had also to be investigated. 
According to many published views on sampling, it is necessary to use probes that are aligned with the flow stream (in these experiments, this would be a probe pointing vertically up the furnace), and then to sample isokinetically (that is, for the gas velocity in the probe to be at the stream velocity). The isokinetic sampling argument is based on the assumption that in non-isokinetic sampling, there will be a separation of particles in the stream, with the smaller particles following the stream lines, and the larger ones not.

In actual measurements over many decades, however, it has been reasonably established that isokinetic sampling is not apparently essential, with results for the most part essentially independent of the sampling velocity. The ostensible reason for this is that the cloud of particles moves substantially as a unit, and the expected in-stream elutriation does not occur. These conclusions are based, however, on fairly gross measurements in successive experiments of sample weight and sample analysis (such as VM content, burn-off, ash percent, and the like). PSD analyses, however, are far more delicate, and from these we have determined some departure from the experimental expectations of the past. This was established in a substantial series of mostly cold-sampling experiments as described in the sections following. The experiments we describe below included re-examination of the isokinetic sampling problem, using conventional across-stream and up-stream probes; but the results also prompted investigation of a new sedimentation probe. Nevertheless, since the results wold have been ambiguous if they 
were affected by the final handling procedures, this was examined first.

5.2 Effect of Solid Sample Handing on PSD Measurements. When the sample has been removed from the flame by the probe and deposited in the filter system, it must be removed from the filter and dispersed in water for PSD analysis. These two steps of removal and dispersion require proper handling. The principal problem associated with sample handing is possible breakage of the particles which yields erroneous PSD measurements. It is this factor that makes PSD measurements so much more sensitive than gross weight of VM determinations used in the past to determine sampling effects.

of the two steps, filter removal and dispersion, the second was judged to be the more sensitive and this was given the greater attention. After removal from the filter or selection of a raw sample, a batch volume of the sample dispersed in water is continuously circulated through the PSD analyser in a closed loop which carries the sample through the analysis cell. Samples used in these tests included "As-received" coal, limestone, flame samples, and ash. Three different methods were used to disperse the samples in the water for the PSD analysis. These were: hand stirring, ultra-sonic mixing, and in-situ mixing by continuous recirculation of the sample in the closed loop.

Figures 5.1, 5.2, and 5.3 in the pages following show the combined results for the three mixing methods, for coal, limestone, and ash samples. These all show plots of $\%$-retained (greater than a given diameter) plotted against (log) diameter. 
In interpreting these results, a shift of the line to the left is indicative of greater fineness in the dispersed sample. The greater fineness in the dispersed sample, however, can be due, either, to better dispersion of the sample in the water (which includes a wetting agent to assist this), or to fragmentation. Distinguishing between the two is not always certain. The reasons for improved dispersion can also depend on the type of sample. In the case of the limestone, both wettability and density can be a problem where the sample left without motion tends to settle rapidly. Consequently, increased fineness of such a sample can be interpreted as greater wetting and dispersion of finer particles, which itself can be due to deagglomertion or breakage. Flame samples, however, are sometimes light enough that without assistance in mixing, surface tension can keep the sample on the surface of the water, even with a wetting agent; and, increased fineness can be indicative either of the finer particles being wetted sufficienty to overcome surface tension and become dispersed in the water volume, or again the result of fragmentation.

For the coal (Fig. 5.1), the results are seen to be almost independent of mixing method, with some small difference between the hand mixing and the sonic mixing. Otherwise, in the top left hand figure (TLH), there is no difference for recirculation times from 1 minute to 19 minutes. Likewise, in the TRH figure, there is no difference between 2 minutes and 15 minutes in the sonic mixture. Only in the bottom figure, as noted, is there evidence of any difference. The conclusion here 
for the raw coal is that either sonic mixing or recirculation in the analyser loop provides good dispersion, but hand mixing should be avoided though the effect is not large.

For the limestone (Fig. 5.2), the picture is a little different. Hand mixing is virtually useless since the sample almost immediately sediments when the mixing is stopped. continuous motion is required to maintain suspension. For the other two methods, the TLH graph shows that the results change with recirculation time up to 2 minutes, though the graphs appear to be converging asymtotically. The continued mixing moves the graphs to the left: thus, after 10 seconds recirculation time, about $70 \%$ of the dispersed sample is greater than 10 microns ( 1 on the log scale), or only $30 \%$ is less than 10 microns; but after 1 or 2 minutes, the greater-than figure drops to $50 \%$ and likewise, the less-than figure rises to 50\%, showing more of the fines have been dispersed. Nevertheless, the asymtotic convergence indicates that this mixing procedure is relatively gentle. The same trend of migration of the lines to the left is seen if only sonic mixing is used (bottom graph), with more fines" progessively dispersed with mixing time. However, comparison with the convergence limit of the TLH graph (shown as 0 time) is indicating that the sample is fragmenting under the sonic mixer if this is maintained for too long a time. Combination of the two methods, however, (TRH graph) with only 2 minutes sonic mixing shows independence of relevant mixing/recirculation times. In view of the difficulty of dispersing limestone under any circumstances, this is an interesting if not important result. 
The ash samples (Fig. 5.3) show different behavior again. In all cases, the ash was so friable that all the dispersion methods caused some-to-substantial degree of fragmentation. Hand mixing was altogether too harsh; the ash so easily fragmented that no results are shown. In the graphs, the left hand graph shows increasing fineness with recirculation time, and this is presently interpreted as breakage since the sample appeared to be fully wetted, and in the water volume, at the start of the test. A similar result is obtained with the sonic mixer. These results suggest that intrusive sampling and batch analysis of the ash PSD will always contain some error although the results shown provide a basis for reducing the error.

In summary, the recirculation appeared to be the gentlest method of mixing, with hand mixing the most harsh, and this handing procedure was therefore eliminated on that account. Recirculation showed varying degrees of breakage or fragmentation, but there was evidence of convergence with time in most cases. A combination of a short period of sonic mixing followed by recirculation may be the best overall procedure. Raw coal is the most robust, and a combination of the two mixing methods is adequate, with considerable latitude in time. The ash samples are the most sensitive, and the procedure adopted was to drop the sample in the recirculator and, after establishing volumetric dispersion, to measure the PSD as soon as possible thereafter. A similar process is used for the limestone, but with the PSD measurement taken after about a minute of recirculation, 
5.3 Probe Design. The most commonly used probe design is a straight water-cooled suction probe and, initially, all solid sample measurements including PSD measurements were to have been made routinely on the samples obtained using such probes; but as results from this procedure were evaluated, concern developed that the samples were not fully representative, at least for PSD determinations. As noted above, some decades of testing the conventional method of sampling had supported the apparent accuracy of the procedure; but in these experiments the PSD analysis subjects the samples to tests of a much higher accuracy and sensitivity than before. specifically, from initial results there was suspicion that the larger particles were being lost in the conventional sampling method. The mass fraction of the larger particles in the total sample is quite small so that VM analysis and internal surface area measurements are not strongly affected, but it does distort the PSD results.

To eramine this possibility of particle loss three different probes were compared. The first was the conventional straight probe; the second was a curved probe so that the probe entrance was pointing upstream at the point of sampling; and the third was a sedimentation probe using sedimentation of the sample on a narrow open tray or slot. Sedimentation probes have been used in the past but were generally not considered feasible or suitable for flame sampling on account of possible further reaction due to continued exposure to the flame before withdrawal; there was also some concern that the dust would flow over the probe, carrying the fine particles and thus inaccurately 
increase the percentage of larger particles. This argument loses force, however, when the mass motion of the full cloud is taken into account.

The results from the three probes were compared with the original sample from the "As-received" coal before dispersion into the air stream, and the results show that the slot probe analysis is evidently closest to the original sample. These results are illustrated on the following pages in Figs. 5.4. and 5.5. These are Rosin-Rammler plots of the PSD results (plots of $\log (-\ln [$ retained/100]) against $\log ($ diameter)). Ideal R-R plots are straight lines. Commonly they are curved as shown but this is often attributed to error in the relatively smaller net proportion of material at the extremes which, on such plots, tend to have undue leverage on the graph line. Nevertheless, comparison of the different curves provides valid bases for determining changes and similarities.

Figure 5.4 is for the curved (upstream) and straight (cross-flow) probes, and it also includes the raw (barrel) samples. For the barrel analyses, samples were taken from the barrel at different depths, from the top to 20 inches down. These results (top graph) are nearly identical, and the differences between the lines are random, thus not supporting the possibility of elutriation by sedimentation (a common problem with large ungraded coal). The two lower gr:phs are then the analyses taken in the cold air stream using the curved probe (Lower Left graph) and the straight probe (LR graph). The different lines are for different flow conditions. The 
differences from the barrel samples are clear. With the curved probe there is, overall, a distinct change in the distribution, with clear evidence in some of the plots of a change to a bimodal distribution. The dependence on flow conditions is clear, however, and this is not acceptable for reliable results. Similar general conclusions can be drawn from the straight probe results: there is not evidence of bi-modality, but the shift to the left compared with the barrel samples is indicative of loss of the larger particles, as often claimed by advocates of isokinetic sampling; however, in this instance, it is more likely to be due to the cross-flow design. The results are also unacceptably dependent on the flow conditions.

These results can be compared with those for the slot probe shown in Fig. 5.5. In this figure, the separate feed and tray samples are shown in the top left hand and right hand graphs, respectively. The tray samples were collected for a range of flow rates, and the notation $30 / 20$, for example, indicates a primary rotameter reading of $30 \mathrm{cfm}$ and a secondary rotameter reading of $20 \mathrm{cfm}$. The raw feed and tray samples are compared in the bottom graph, and the agreement is excellent. However careful analysis of a large number of tray samples (approximately 30 total) showed that the smallest particles (usually representing less than $10 \%$ of the volume) could be lost in the tray sampling method.

5.4 PSD Analysis of Coal/Limestone Samples. With limestone in the flame, the sample then is a mixture of limestone and coal or char. Determining the PSD of the mixture is no problem so long 
as it properly disperses in the carrier water for analysis. Interpretation of the analysis, however, is another problem. Ideally, the two constituents should be separated; this is needed to be able to interpret the behavior of the coal/char kinetically. Separation, however, is a problem that is as yet unsolved. In this section we describe the experiments and their results in attempts to separate the two solids. First, however, we note the results of an alternative approach to calculate the PSD of a mixture. This alternative was to determine the agreement between calculation and measurement for the PSD of a mixture.

The results of comparing calculation with measurement are illustrated in Fig. 5.6. The argument here is that if the joint PSD can be calculated, and if the limestone PSD remains essentially unchanged through the flame, then the char PSD can be obtained by back-calculation from the mixture PSD. In Fig. 5.6, the PSD values are shown in a different representation as this is the best for the comparisons required. The graphs in this figure are plots of $\%$ by volume in a given size range plotted against (log) diameter. Consequently, these are differentials of the cumulative distribution. In this figure, the TLH and TRH graphs are for the coal and limestone respectively. Plotted this way, the graphs show the location of the peak of the distribution; and, so doing, show also the very different fineness of the two samples: these are about 50 and 5 microns, respectively. The bottom two graphs then show the measured and predicted distributions for a 3:1 mixture. The agreement is so close that 
they have not been plotted on the same graph as they then are essentially indistinguishable. Even so, the effect of the limestone addition is still noticeable by comparing the top and iottom LHS graphs: the peak remains at almost the same location, but its magnitude is clearly reduced, from 17 or $18 \%$ to about 15\%, and the graph is clearly broadened. Correspondingly, if the mixture ratio and the limestone PSD are known then the coal/char PSD can be determined by difference. The key to this, however, is determining the limestone proportion in the mixture.

This problem of separation has yet to be solved, and the methods examined identify some of the difficulties to be resolved. The first method attempted was physical, by centrifuging (nominally possible since the density of limestone is about 1.6 times that of coal, and this would be higher still after pyrolysis or partial combustion). Centrifuging did provide quite a high degree of separation, but still inadequate for the intended purpose. The second method was chemical, using $\mathrm{HCl}$ to dissolve out the limestone. This did satisfactorily remove the calcium carbonate, but this particular limestone contained sufficient magnesium compounds that full removal was unsuccessful. The alternative to removing the limestone is removal of the coal/char. This can be done by burning it out but there are problems with this as well; the most important is that removal by combustion still leaves the coal ash as an uncertain additional factor.

In summary, PSD's of mixtures can be obtained; but interpretation in terms of changes, separately, of the coal/char 
and limestone requires separation or separate determination of the limestone/combustible ratio, and successful separation or ratio determination will require futher work.

5.5 proximate Analysis of Samples containing Limestone. This is a closely related problem to the one of sample separation for PSD analysis, with further significance for the determination of the reaction efficiency. Reaction efficiency from the solid samples is conventionally obtained by determining the ash content of the sample, by heating under preset (ASTM) conditions in an ashing oven, and calculating the ratio of combustible to ash. This ratio is then converted into reaction efficiency.

The standard (ASTM) method to determine the coal ash content is to gradually heat the sample and then maintain it at 700 to $750 \mathrm{C}$ for coal, and not more than $950 \mathrm{C}$ for coke, for two hours. Although this method is the ASTM standard and in consequence is widely used, it is not satisfactory if the coal or char sample contains a high percentage of carbonates or pyrites as part of the mineral matter since their decomposition is very sensitive to temperature variation and heating time; it also misstates the actual sample temperature since the prescribed temperature is that of the furnace, and the process of ashing can increase the sample temperature to varying degrees depending on the quantity of combustible, and this can affect the extent of decomposition of the minerals.

This method is also not satisfactory for samples containing limestone since, like the mineral matter carbonates, they decompose to a greater or lesser degree both in the flame 
and in the ashing process, and this has to be accounted for in the decomposition and reaction efficiency calculations. Consequently, an alternative method had to be developed for measurement of the ash content of a solid sample containing limestone.

Limestone is subject to decomposition at temperatures over $600 \mathrm{C}$, with the decomposition considered insignificant for temperatures lower than $600 \mathrm{C}$ and complete for temperatures over $1050 \mathrm{c}$. In slow decomposition, the extent of the limestone decomposition is determined by the partial pressure of $\mathrm{CO} 2$ which increases exponentially with temperature. In fast heating in short times, as in a flame, the extent of decomposition is also a kinetic process so that only partial decomposition may have occurred in the flame even when the temperatures are high.

In the proximate analysis, the temperature programming as already described requires ashing oven temperatures at least up to $700 \mathrm{C}$ for coal, and the sample temperatures can $r i s e$ substantially higher from the combustion of the combustible, and possibly as high as $1400 \mathrm{C}$. Thus partial or complete decomposition of the undecomposed limestone will occur during the ashing, and this raises obvious difficulties in determining the ash content of the furnace sample and, consequently, in - determining the combustion efficiency and sulfur capture efficiency. In summary, the problem is twofold: first, the extent of initial limestone decomposition in the flame is unknown, at the position where the sample was extracted; and second, further partial or complete decomposition of the 
limestone will occur during the standard ashing technique itself, and the extent of this is also uncertain.

In designing procedures to solve this problem, one solution considered but discarded for lack of equipment and time was the Low Temperture Ashing (LTA) technique. This uses an RF pulse to oxidize the organic material in the coal at a very low temperature (150 to 200 C) in a vacuum chamber. This temperature is so low that the mineral matter is not converted to ash, and it is particularly valuable on that account when information on the mineral matter content and behavior is required. Consequently, the method is mainly used to see the variations of the elements and structures in the coal during the slow process of oxidation. It is also favored when possible for analyzing western coals which commonly have higher percentages of alkalies that can vaporize at higher temperatures. The drawback of the ITA technique, however, is that it is not generally regarded as practical for large numbers of samples because it is so time consuming, usually requiring several days for analysis because of the slow reaction rate at the low temperatures.

As an alternative, we examined the other approach of very high temperature ashing, by driving the combustibles off at temperatures above $1050 \mathrm{C}$ so that the decomposition of calcium carbonate is complete. In Table 5.1 we show the comparative results between the standard ASTM procedure and a high temperature variant of the standard method. In this variant, the method starts with the ASTM method. The sample is heated in the ashing oven in the presence of adequate air, first to 450 to 500 
$C$ in an hour, and then to 700 to $750 \mathrm{C}$ by the end of the second hour. The temperature is then maintained within this range for an additional two hours. These steps are the same as the standard method. In the high temperature extension, the sample is furthur heated to 1050 to $1100 \mathrm{C}$ in one hour and maintained at that temperature for an additional hour to ensure complete decomposition of calcium carbonate. The sulfates and sulfides may also be decomposed, and some alkalies may be vaporized; however, this should be negligible as long as the temperature does not exceed $1100 \mathrm{C}$. Carbonate (and other minerals) will decompose at 700 to $750 \mathrm{C}$ in the standard method, but the extent of decomposition is uncertain, as already noted. With the high temperature ashing technique the decomposition is complete. The net weight after loss is the ash content.

In the results shown in Table 5.1, there were 4 paired sets of tests in all, the first pair using the standard ASTM procedure, and the other 3 using the extended procedure. In each set of paired tests, the samples were analysed together in the ashing oven so that they were known to have had essentially the same temperature/time history; this is otherwise very difficult to achieve, even with very expensive computer-controlled ashing ovens. Three samples were examined in each of the 4 paired tests for repeatability. As shown in the Table listing, Test \#1 (standard method) examined coal and limestone. These were also used, for first comparison, in Test \#2. Test \#3 was for coal and a 3:1 coal/limestone mixture; and Test \#4 was for limestone and the same $3: 1$ mixture. 
In the first test, (Table 5.1) the weight loss of the' coal (ash content) is very reproducible, as expected from the standard test, and the variation is well within expectation for these measurements. This is not the case for the limestone, however. Although all three limestone samples were in the oven simultaneously, the variation of weight loss (carbonate decomposition) is quite striking and is attributed at this time to temperature variations in the oven.

With the temperature increased in the extended test method, Test \#2 shows altogether more consistent results for the limestone. The results are also consistent for the coal, though there is a further loss of about $0.7 \%$ of the original weight as compared with Test \#1. This could very well be removal of part of the residual VM matter that is known to be up to $2 \%$ and consists commonly of about $1 \%$ hydrogen and $1 \%$ nitrogen. This increased loss, however, is considered unimportant in this context.

In Test \#3, the coal results are again consistent with those from Test \#2. The expected result from the mixture is about a $22 \%$ residue so the measured value of about $25 \%$ is a little high, but close to expectation. The $3 \%$ difference could well be due to high temperature formation of high temperature sulfates that are not decomposed, by binding between the limestone and coal mineral matter constituents. Proof of this conjecture, however, will require extensive chemical analysis and must be reserved for later investigations. At this time, the agreement is adequate for the purposes here. 
In the final test (\#4), the limestone data are essentially shown to reproduce the results of Test \#2, and the mixture data reproduce those of Test \#3.

In conclusion, therefore, we accept that these results show that the ash content is determinable using the hightemperature (extended ASTM) procedure. Using this method, the calcium carbonate decomposes completely so that an inert base is provided for determination of the combustion efficiency. 
Table 5.1: Weight Loss of Coal, Limestone, and Mixtures in High Temperature "Ashing" Tests: Figure is "Ash" Percent

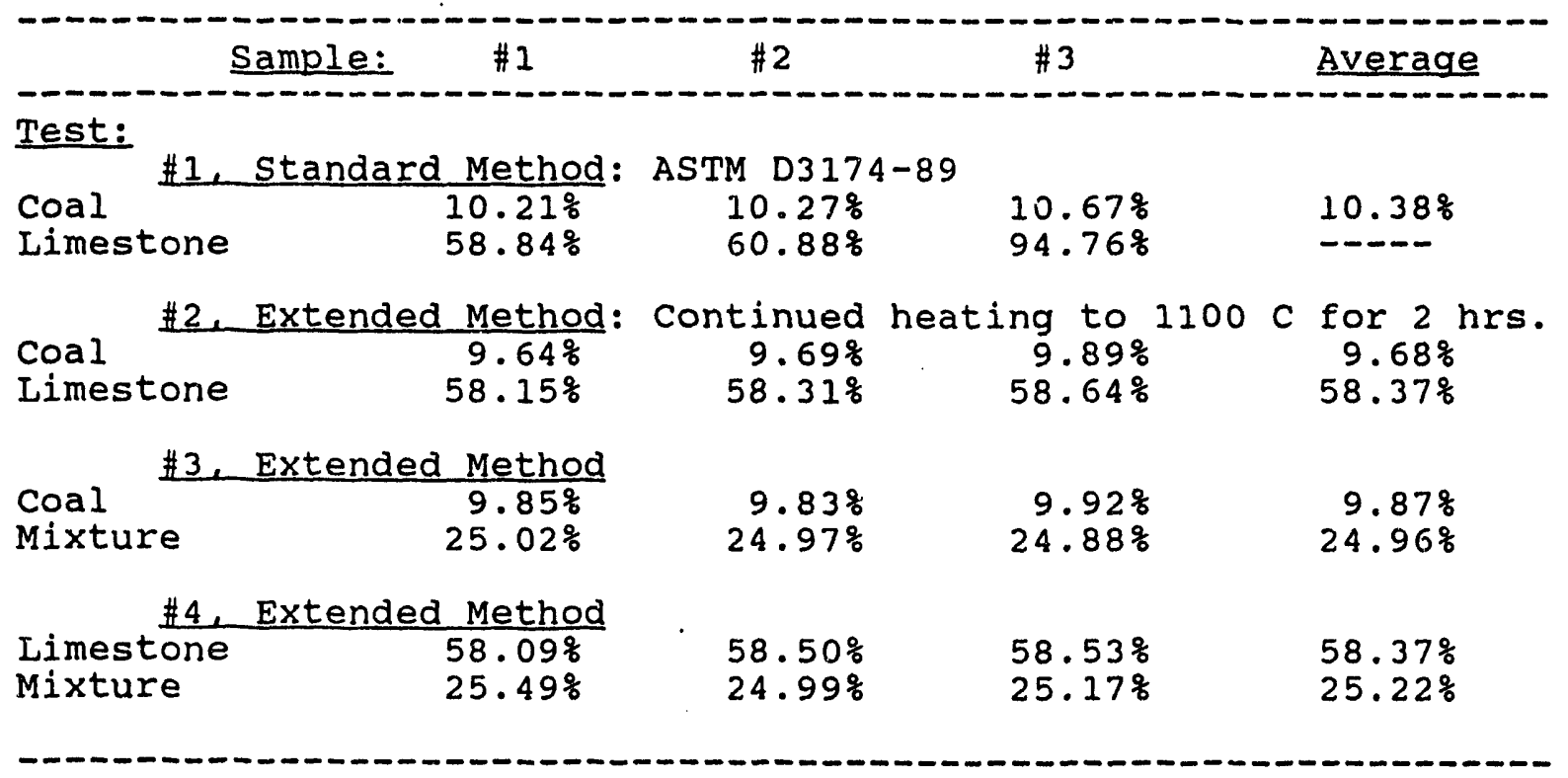




\section{PSD: COAL SAMPLES}

INCREASING RECIRCULATOR TIME

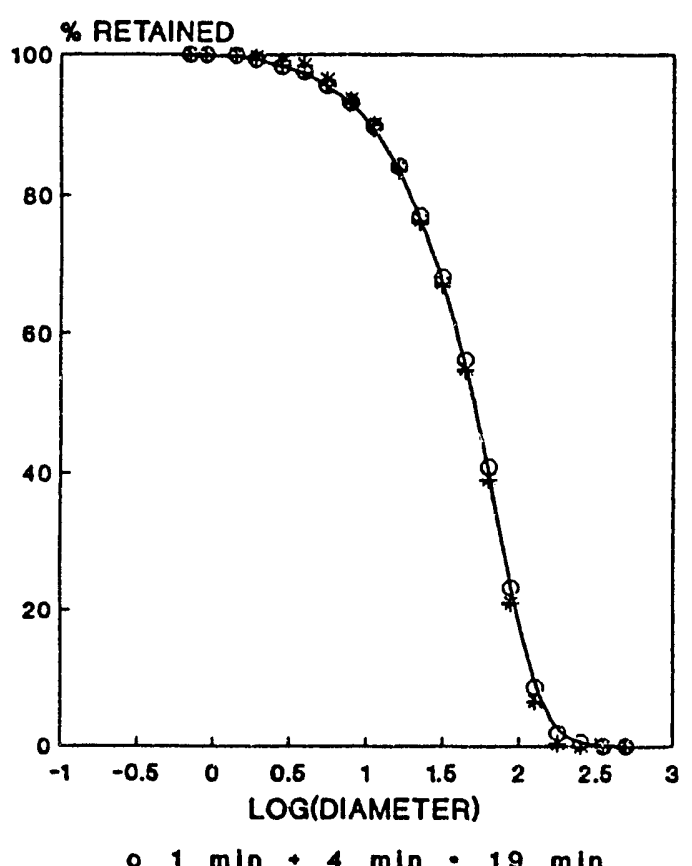

EFFECT OF SONIC MIXER

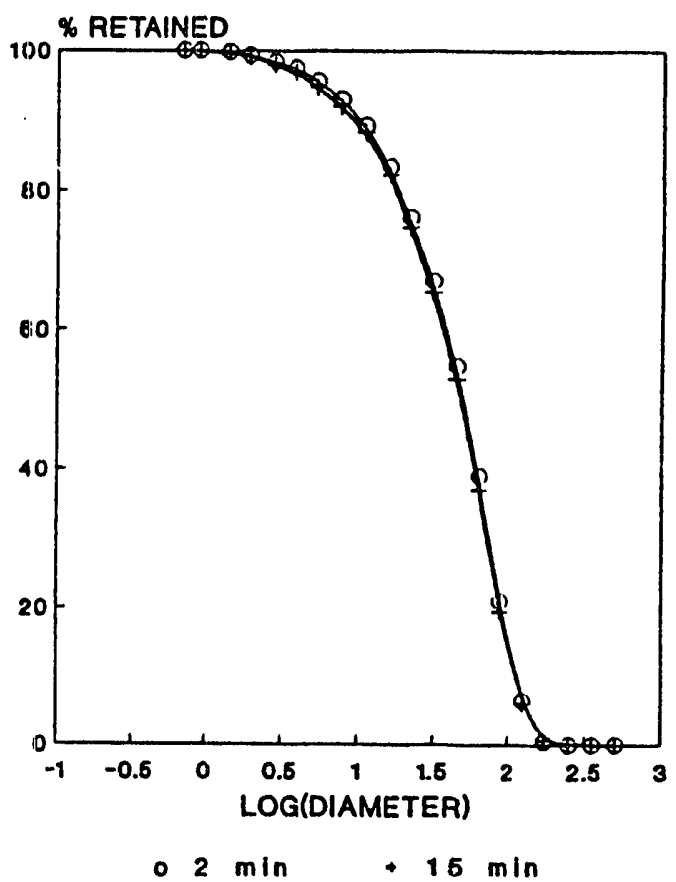

EFFECT OF HAND MIXING

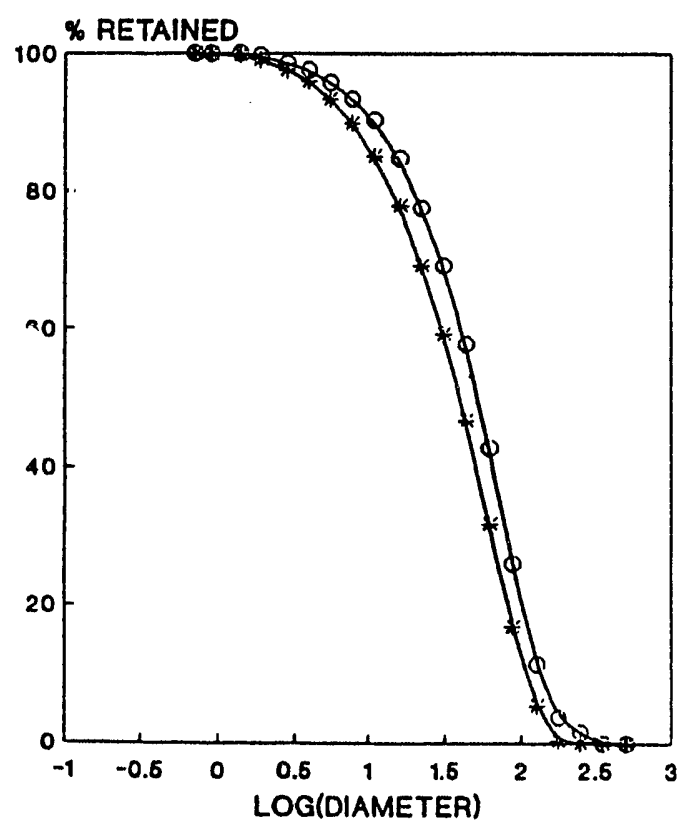

o sonic $m i x$. hand $m / x$

FIG. 5.1 


\section{PSD: LIMESTONE SAMPLES}

INCREASING RECIRCULATOR TIME

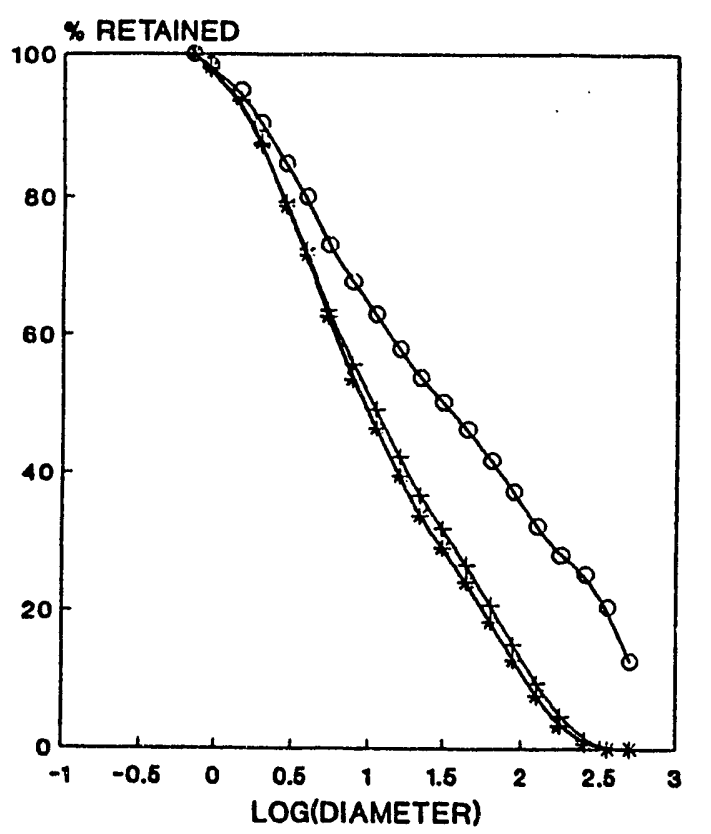

NO SONIC MIXING
$010 \mathrm{sec}+1 \mathrm{~min} \cdot 2 \mathrm{mIn}$
INCREASING RECIRCULATOR TIME

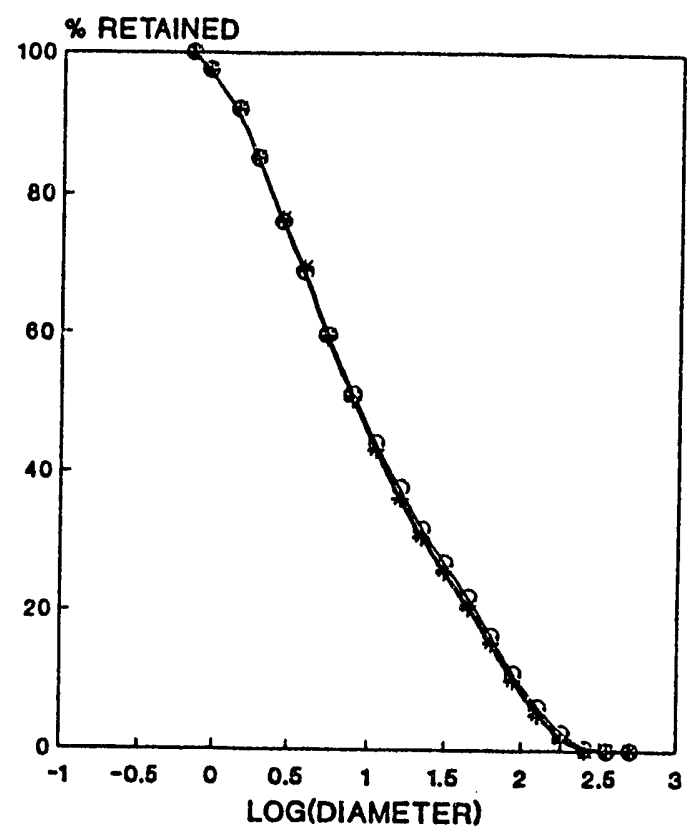

2 MIN SONIC MIXING

- $3 \mathrm{~min}+14 \mathrm{~min} \cdot 21 \mathrm{~min}$

EFFECT OF SONIC MIXER

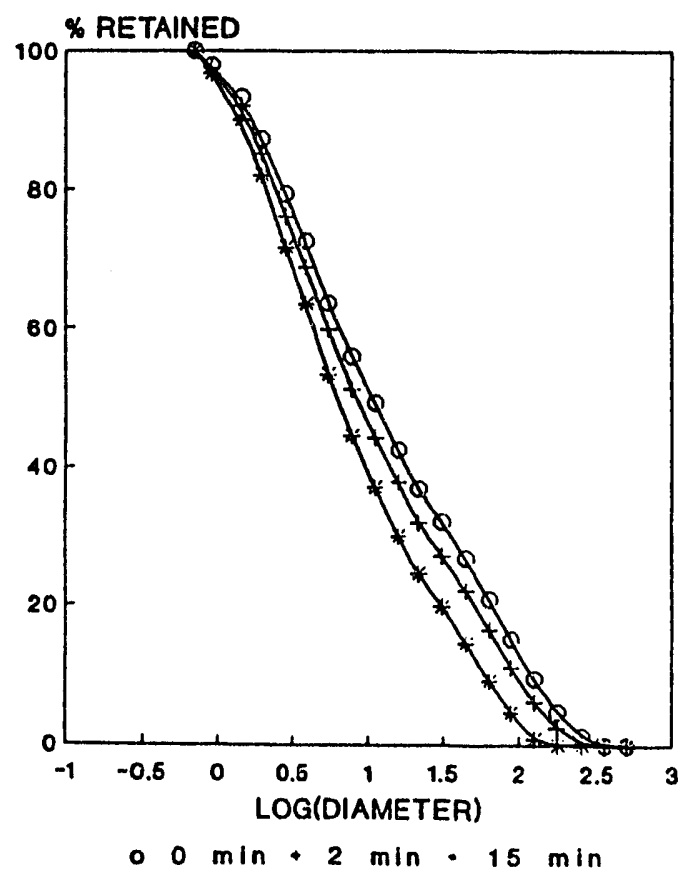

FIG. 5.2 


\section{PSD: ASH SAMPLES}

INCREASING RECIRCULATOR TIME

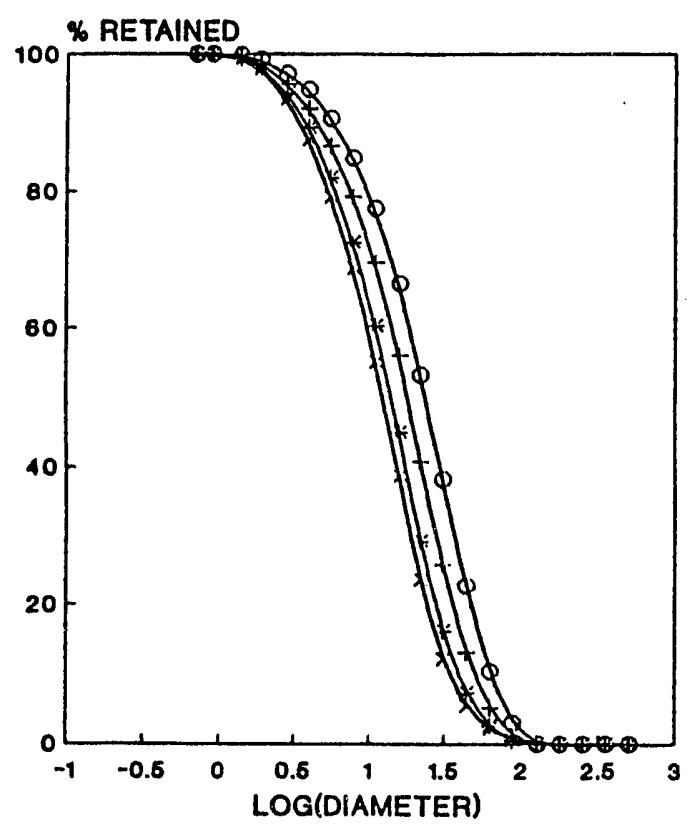

$01 \mathrm{~m} / \mathrm{n}+2 \mathrm{~m} / \mathrm{n} \cdot 8 \mathrm{~m} / \mathrm{n} \times 19 \mathrm{~m} / \mathrm{n}$
EFFECT OF SONIC MIXER

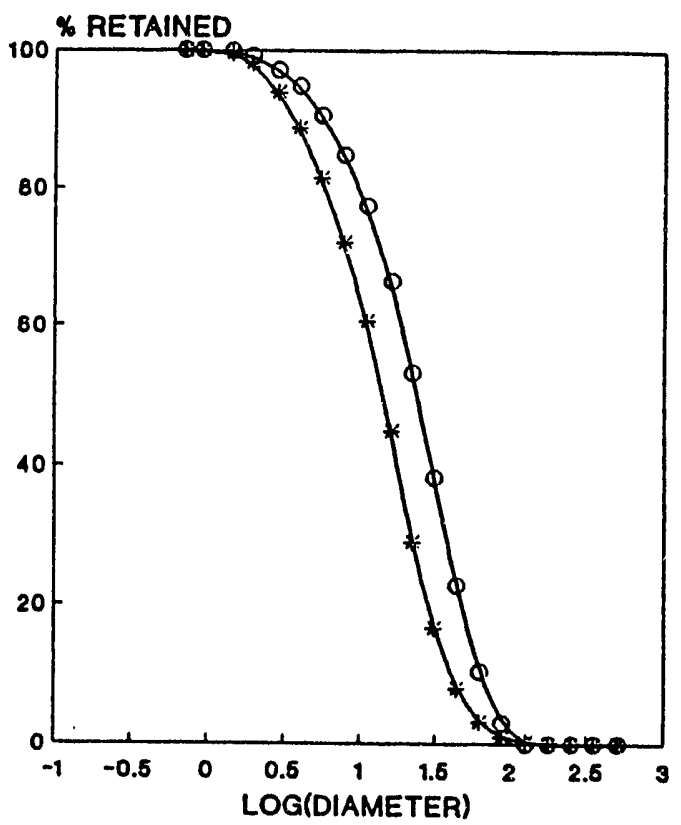

- $1 \mathrm{~min}$ reclr. - sonlc mixar

FIG. 5.3 


\section{PSD: CURVED AND STRAIGHT SUCTION PROBE SAMPLES}

\section{SAMPLES FROM BARREL.}

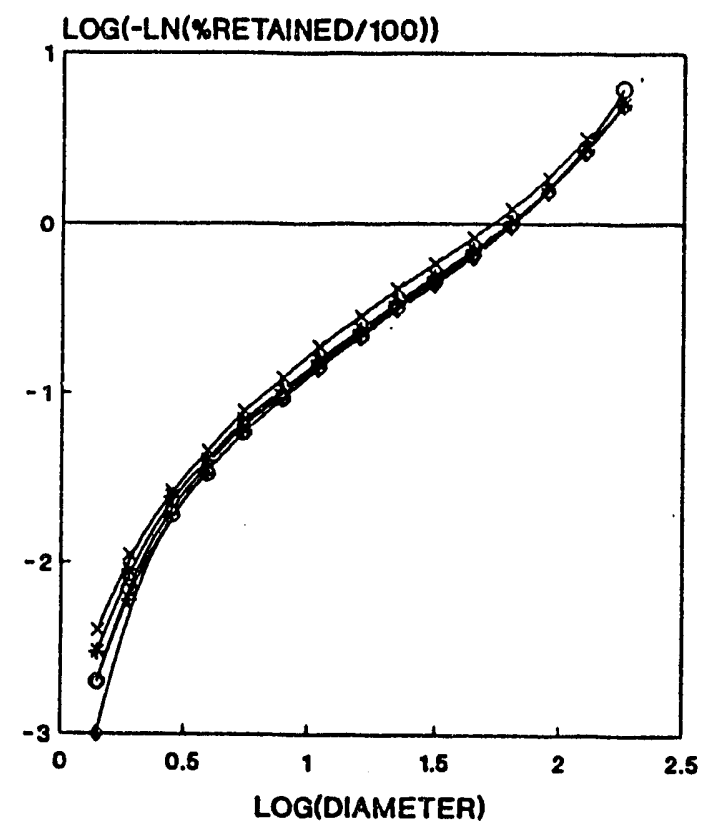

CURVED PROBE

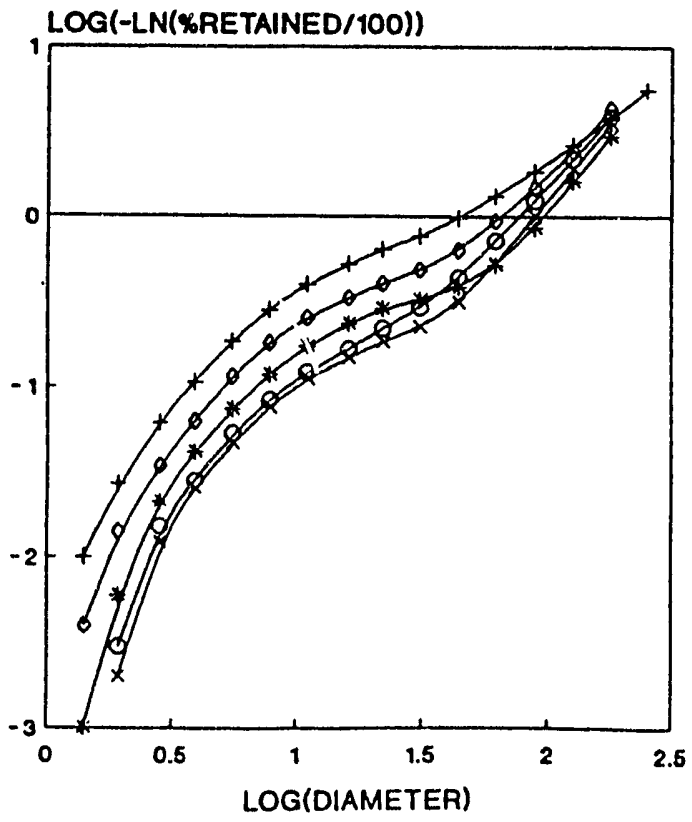

STRAIGHT PROBE

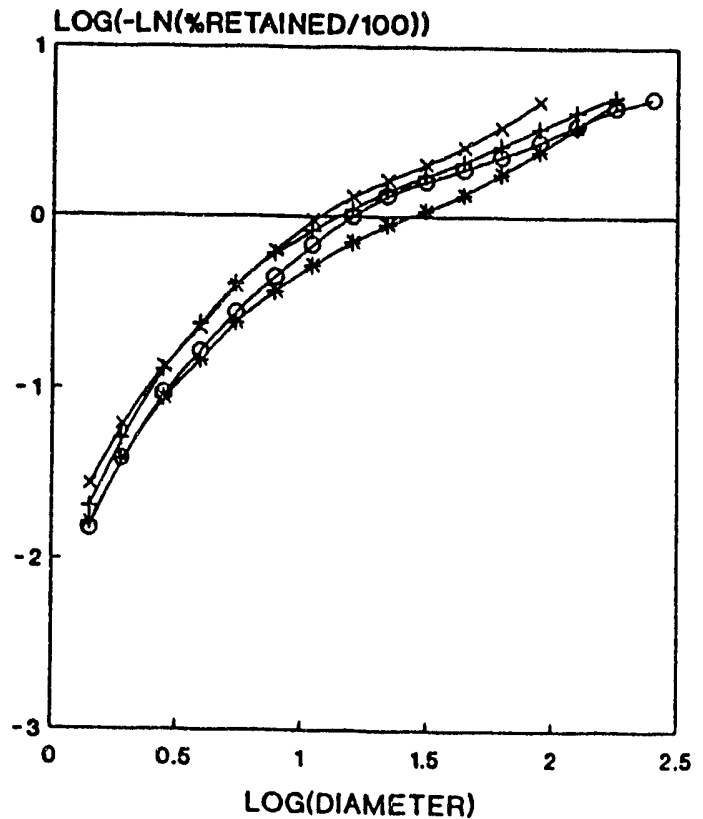

FIG. 5.4 


\section{PSD: TRAY SAMPLES}

FEED

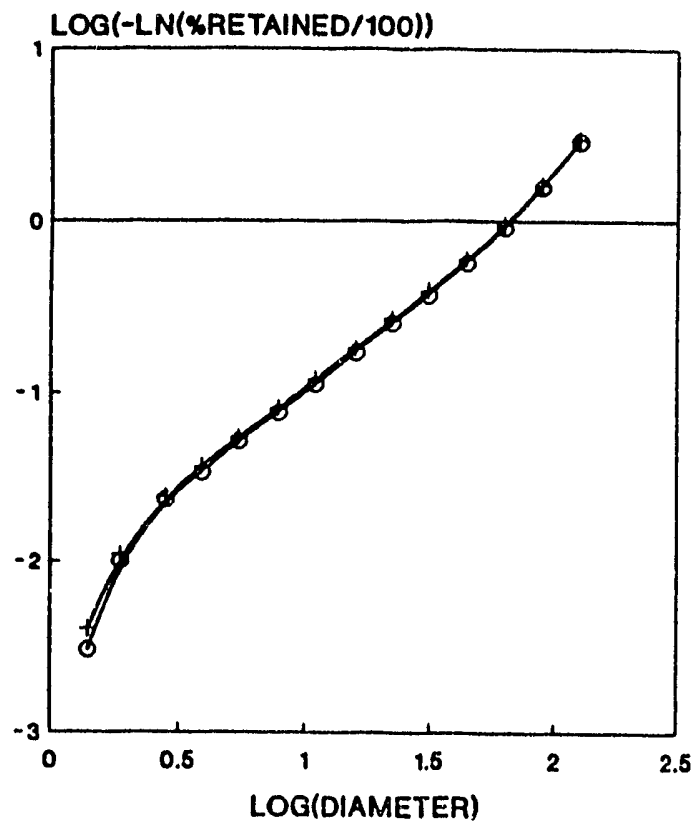

TRAY SAMPLES

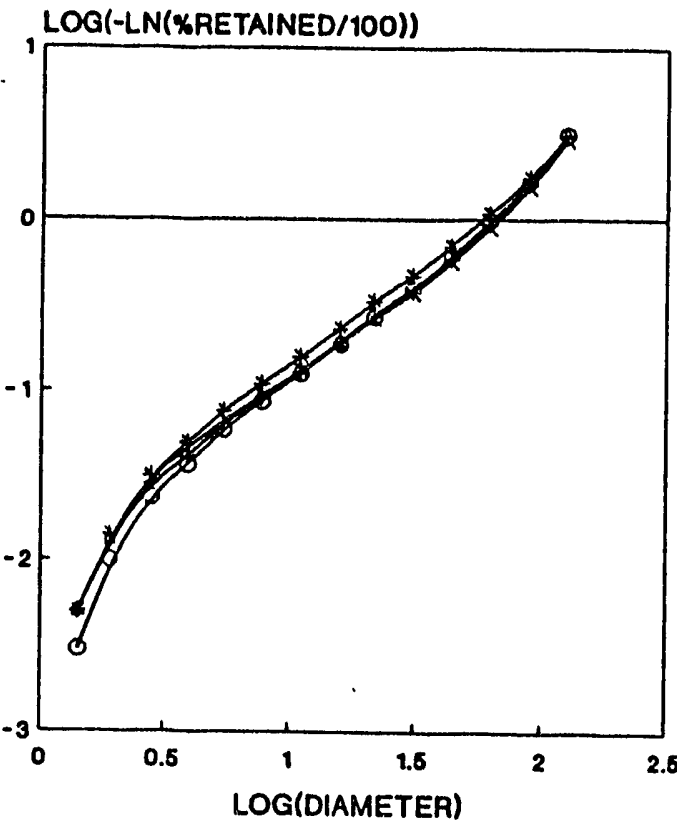

$\begin{array}{rr}-30 / 20 & 30 / 10 \\ -10 / 15 & 010 / 5\end{array}$

$\times 10 / 20$

FEED AND TRAY SAMPLES

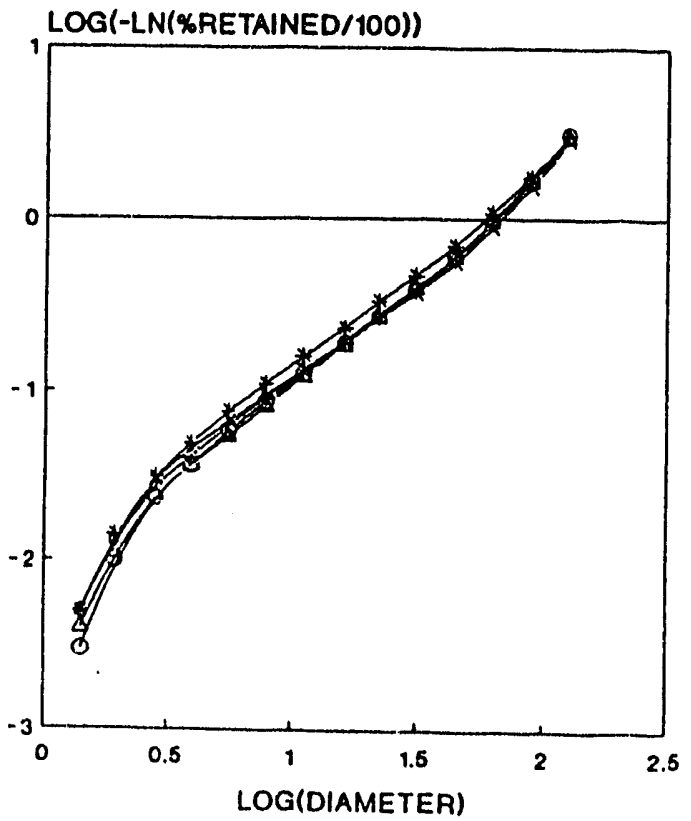

$\wedge$ Feed

FIG. 5.5 


\section{PSD: COAL, LIMESTONE, AND MIXTURE}

COAL SAMPLES

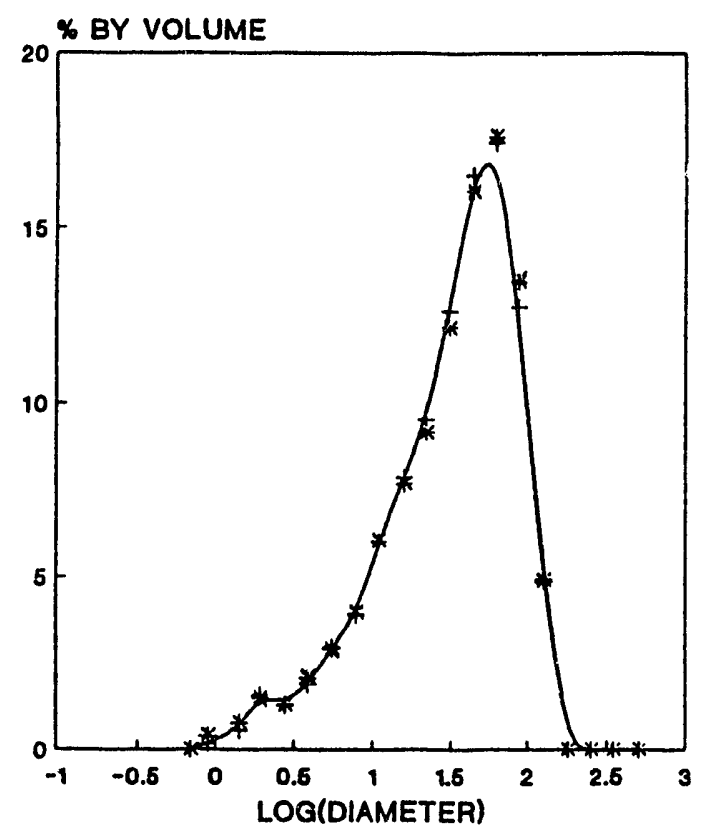

3:1 MIXTURE

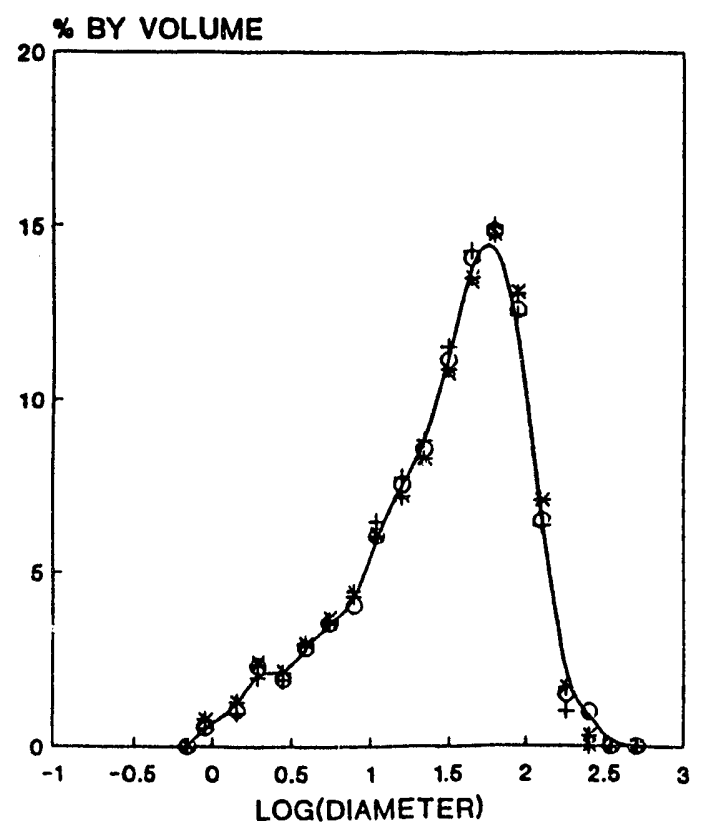

LIMESTONE SAMPLES

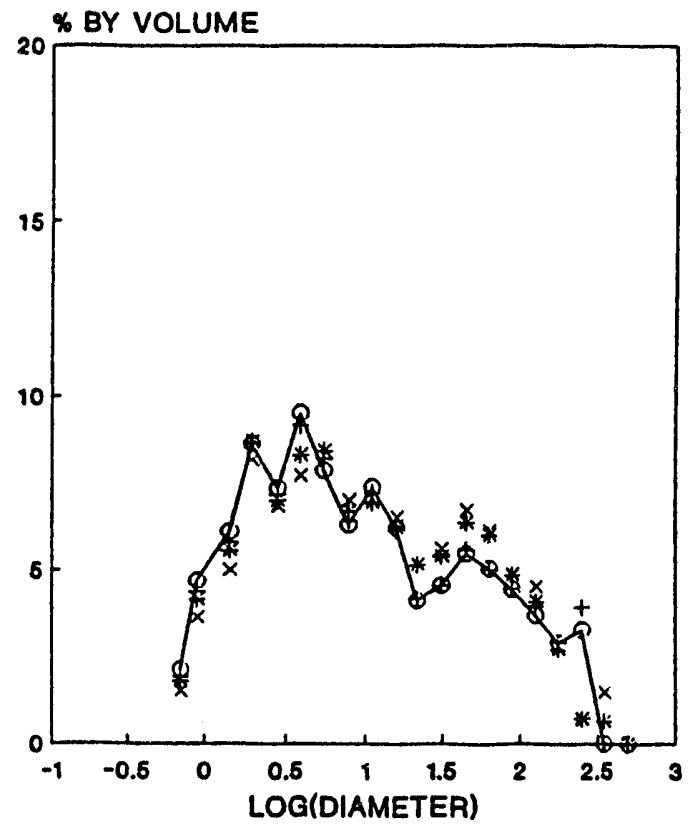

PREDICTED 3:1 MIXTURE

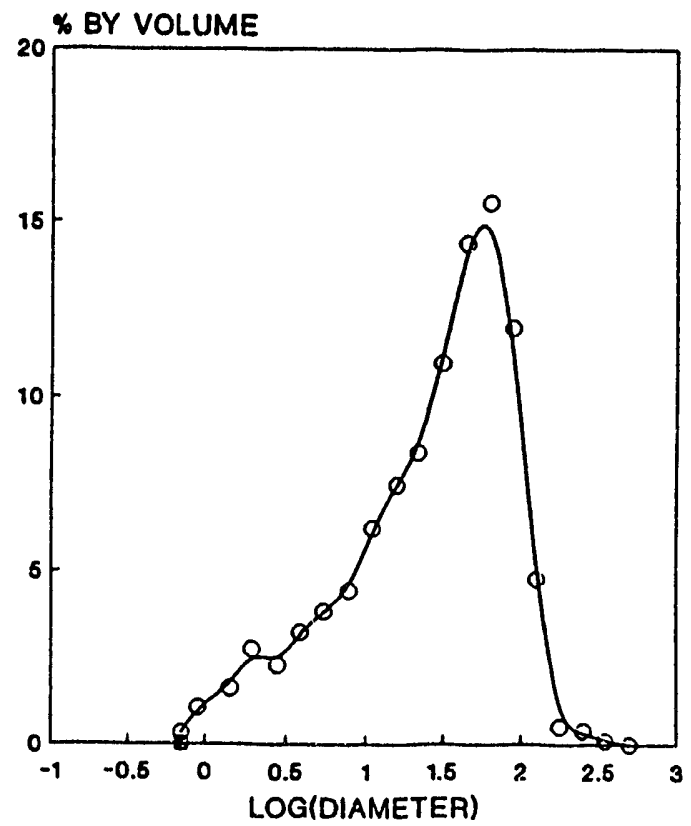

FIG. 5.6 


\section{RESULTS III: FLOW STRUCTURE INVESTIGATIONS}

The results described in this section are for mostlyexperimental investigations carried out when the problem of possible non-uniformity of the horizontal profiles and the alternate (cone-shaped) flame structures became evident. The principal focus was on the flow structure with most emphasis on the physical factors such as the velocity profile that could affect the flame structure. In pursuing these investigations they were carried out in a logical question-answer-question sequence. However, the order in which the results are presented here is not the order in which they were executed but in the order that seems to make best sense of the results obtained.

There are two groups of results: the first focuses on the physical (cold-flow) structure of the flow and the second on the hot-flow profiles. These results shown in the figures are a sinall sub-set of all the results and have been selected as the most significant or informative for the purpose of the investigations.

6.1 Cold Flow profiles. These results are illustrated in Figs. 6.1 to 6.9 , and include experiments and calculations on factors including the boundary layer growth, particle acceleration and velocity, and dust cloud uniformity. The background to all these considerations was the evidence that the flame could be coneshaped, and one possible reason for this was that it might be determined by the physical characteristics of the flow. The overall conclusion at this time is that these factors are not, in 
fact, the source of the different flame types. Nevertheless, excluding these as significant factors is of major importance.

6.1 .1 Boundary layer thickness. The boundary layer thickness was a factor given late attention and, as the results show, it does not seem likely to have had any signicant effect on the flame pattern. Nevertheless, the results are instructive, and important in their own right.

The results of the calculations are shown in Fig. 6.1 and in Table 6.1, and they focus on the boundary layer development and entry length for different flow regimes. The entry length is the distance over which the flow, assumed planar at entry, has reverted to its fully developed profile for the particular flow conditions in question. The values in Table 6.1 are given for three air rates (the same three air rates as used in cold gas velocity tests described below) and for two temperatures: $T=300$ $\mathrm{K}$ and $\mathrm{T}=1500 \mathrm{~K}$. In calculating the entrance lengths, laminar flow was assumed for purposes of comparison although the Reynold's number for some of the flows exceeds 2000 . Also for the purpose of comparison, the boundary layer thickness at a furnace length of 2 meters is given. This was estimated assuming linear growth of the boundary layer which is a commonly-used first-estimate, but is, as shown in what follows, somewhat conservative compared with other calculations since the boundary layer growth using other calculation methods is non-linear. The Table values show that the boundary layer thickness decreases for higher flow rates and increases for higher temperatures. The majority of the values, however, are less than $1 \mathrm{~cm}$ at a location 
$2 \mathrm{~m}$ downstream, in a duct $16 \mathrm{~cm}$ across. At entry and in the first $50 \mathrm{~cm}$ of the flow, which are crucial in forming the shape of the flame front, the BL thickness is essentially trivial.

An alternative treatment shows rather more possibie effect. This is shown in Fig 6.1, which shows the development of the boundary layer along the furnace duct using the Blasius layer calculation for laminar flow over a flat plate for which there is a readily availabie solution. The calculations have been carried out as before for the same cold and hot temperatures, and for the same flow rates as in Table 6.1. Hence, three boundary layers are shown in each figurc, for the three flow rates. The plotted boundary layer thickness is the distance at which the velocity is $90 \%$ of the freestream velocity. Each plot shows the variation of the (horizontal) boundary layer thickness as it develops along the furnace, shown as vertical distance, for a length in the furnace up to two meters.

These plots show more influence of the boundary layer than in the linear extrapolation estimation and can be quite substantial compared to the internal horizontal width of the furnace (0.165 meters). This calculation does not take into account the effect of the pressure gradient created by the presence of the other walls, however, which thus over-estimates the BL thickness since the Blasius solution is for a laminar boundary layer on a flat plate with zero pressure gradient. Consequently, the BL thickness for the actual conditions in this furance will lie between the two estimates. 
Although these figures show that the $B L$ build up as calculated from the Blasius solutions occurs in the first $50 \mathrm{~cm}$ or so, nevertheless, in this distance which is crucial in forming the flame front, the layer is still only a centimeter or so. If we accept that the real value lies closer to that from the calculation from the entrance length, as shown in Table 6.1, then the true value will be even smaller. Consequently, it is reasonable to conclude that any variation in the horizontal gas concentration profiles is not likely to be due to the presence of boundary layers along the furnace walls, where the boundary layer thickness is unlikely to be more than about one centimeter at a distance of 2 meters from entry for the most commonly used flow rates.

6.1.2 Relative particle velocity. A factor related to the flow stream velocity and to the possible influence of the velocity variations in the boundary layer is the relative velocity of the particles through the flowing gas. To estimate this we assumed, for purposes of calculation as a limiting condition, that the particles were injected into the flowing stream at the furnace entry, at initial velocity $v_{p}{ }^{0}$, and then accelerated under the influence, jointly, of gravity and friction (buoyancy can also influence the system but this is ignored at this time as it is a macroscopic effect and this evaluation is only for the microscopic factors of the single particles). For such a system there are well known equations and solutions. For a particle of mass $m$ and diameter d moving downwards at a 
variable velocity $v_{p}$ through the gas (at temperature $T$ ) which also is moving downwards at (steady) velocity $v_{g}$, then the relative velocity is $v$ which determines the drag force. The DE that describes the acceleration of the particle is then

$$
d v_{p^{\prime}} d r+3(\rho \mu d / m) * v_{p}=\left[g+3(\rho \mu d / m) * v_{g}\right]
$$

where the RHS is constant. The solution is a [ 1 - exp] rise in the form

$$
v_{p}=v_{p}(\text { final })+\left[v_{p}(\text { final })-v_{p}^{0}\right] \cdot \exp (-t / r)
$$

where the time constant for equilibration is given by

$$
T=m /(3 \rho \mu d)
$$

and $v_{p}$ (final) is given by

$$
v_{p}(\text { final })=v_{g}+g * r
$$

The solution shows that the final velocity exceeds that of the gas but the calculations show that the difference is trivial under flame conditions. The other important factor is the time constant for equilibration which is fractions of a second. The overall behavior is illustrated in Fig. 6.2 where the particle velocity has been calculated for a range of 
different diameters, from 20 to 150 microns (at 20, 40, 60, 80, 100, and $150 \mathrm{microns}$ ), in both cold and hot flow (at $300 \mathrm{~K}$ and $1500 \mathrm{~K})$, and the results have been plotted against time. To evaluate these time scales, the ignition time is about 0.1 second in this furnace. In these plots, the initial velocity is taken as zero, as the most severe limiting condition, and the ambient velocity is taken as 0.5 and $2.5 \mathrm{~m} / \mathrm{sec}$ in the cold and hot flows respectively.

The results show that the equilibration in cold flow takes from 0.01 sec. up to about 0.2 sec. for the two extreme particle sizes, and the terminal velocity can be 10 to $100 \%$ greater than the gas flow. In the hot gas, however, on account of the increased drag due to increased viscosity the time constant becomes even smaller so that equilibration is achieved substantially before ignition and, equally significantly, the difference from the ambient gas velocity at equilibration is insignificant even for the largest particles.

From this we conclude that the individual particle response is generally so fast that we should not expect any significant difference between the velocities of the particles and gas. In actuality, moreover, since the particles tend to move in groups rather than as individuals, even the small velocity differences shown in these calculations are not expected to exist.

6.1.3 Measured particle velocities. To check the conclusions of the previous calculations as far as possible, the particle velocities were measured using the Insitic PcsV 
instrument. As discussed in Sec. 2.5.4, the instrument monitors particles passing through the sample volume one at a time so that reliable results require quite long sampling times to get sufficient measurements for large-number statistics required for accuracy. One problem arising from this is that the sampling times can be long enough, particularly in flames, that feed and flame conditions can change detectably during sampling.

The results are shown graphically in Figs. 6.3 to 6.5 . All measurements reported in these graphs are in cold flow so that there is best chance of comparing with the calculated flow velocities. The first two tests were to check the relative results in cold flow at different vertical locations in the furnace, and then at a set location for a range of air flow rates. These results are shown in fig. 6.3. The remaining tests were to check for flow uniformity by measuring the velocities at various positions through a horizontal cross section of the furnace and the results are shown in figs. 6.4 and 6.5 . In all the horizon graphs, the $x$-axis scale shows the overall, outside dimension of the furnace (12 inches) so that the inside walls are. at 2.75 and 9.25 inches respectively.

For the first test the air flow velocity was set at 1 $\mathrm{m} / \mathrm{sec}$ and, as shown in the LH graph of Fig. 6.3, the measured velocities at the different vertical positions are all roughly $80 \%$ of the calculated flow velocity. It is not certain, however that this discrepancy is due to particle slip and, indeed, it is unlikely to be so, arguing from the speed of equilibration of the particle velocities shown in the earlier graphs. The 
calculations show that the particle speed exceeds the gas at equilibration and, up to that point, the particles are still accelerating. Accordingly, we should expect to see that, if the difference is real, then it should be diminishing with time (or distance down through the furnace shaft), and this does not happen. These results show that there is no evident change in velocity, i.e., acceleration between these three vertical positions.

The difference in measured and calculated velocities is also clear from the $\mathrm{RH}$ side graph of fig. 6.3 which is a plot of measured against calculated velocity for a number of other air flow rates. This graph shows that there is a systematic difference between the two velocities. Error bars for the mean points are not shown but a common line goes through the means inside the error bars showing that the difference is consistent. The difference shown is believed to be systematic error and is most probably due to the limited range of possible velocity measurements for a given TAC (Time Amplitude Conversion) range which much be chosen and set in the signal processor prior to each measurement. If the actual average particle velocity is near either the upper or lower limit of the TAC range, some of the velocities (in the tails of the bell shaped distribution of velocities) are not within the TAC range and are therefore not counted in the average. This means that the measured value for an actual average particle velocity near the lower limit of the TAC range will be slightly high, while the measured value for an actual average particle velocity near the upper limit of the TAC 
range will be slightly low. If we read the $\mathrm{RH}$ graph and the rest of the graphs in figs. 6.4 and 6.5 in this way then these show substantial agreement with the calculated gas velocity.

Horizontal profiles are shown in Figs. 6.4 and 6.5 . Tilese are for various flow rates and settings of the TAC range and discriminator. In each figure, the upper graph(s) is(are) at TAC $50 \mathrm{msec}$ and the lower ones are at TAC $20 \mathrm{msec}$. The discriminator settings are variable. Again, in each graph, the solid line is the calculated velocity (assumed constant acruss the profile). The measured values for the highest flow sate (about $1.1 \mathrm{~m} / \mathrm{sec}$ ) are again all roughiy $80 \%$ of the calculated value, while the measured values for the lowest flow rate (about $.2 \mathrm{~m} / \mathrm{sec}$ ) are higher than the calculated value. This is most likely due to the limited range associated with the IAC setting as previously described. The profiles, overall, are Feasonably constant and show no particularly marked variation that is not inside the overall error of measurement.

The conclusion at this time is that fo: the measurements made, the particle velocities across the horiznntal are reasonably constant within the limits of experimental error. In addition, there is no evidence of necsurable slip. Discrepancies are believed to be systematic errors due to the limited range of possible velocity measurements for a given TAC setting.

6.1 .4 Air velocity profiles. Background to the particle velocities is the profile of air velocity. This was investigated using a hot wire anemometer since the velociries were too low for a pitot. Hot wire anemometers are notoriously sensitive so that 
profiles commonly show quite wide variations or fluctuations that are substantially smoothed out by sufficient repeats. In these experiments, time did not permit large numbers of repeats so that the profiles are unsmoothed. All measurements made are horizontal profiles, at different horizons, different flow rates, and with different wall. conditions. There was also some variation in the way in which the air was introduced into the cone. The results are shown in Figs. 6.6 to 6.8 . Since the total length of the hot-wire probe was only 8 inches, this allowed probing only about 80 or $90 \%$ across the flow $(5-1 / 2$ to 6 inches) from either side so that the profiles given typically show the boundary layer profile for only one side.

Figure 6.6 gives the profiles at ports 5 and $10(.42$ and $1.35 \mathrm{~m}$ below the tube bank respectively) with only the boundary layer (shown by the drop in the velocity profile) on the right side showing clearly because of the hot-wire probe length limitation described above. These measurements were made following a combustion experiment in which a considerable quantity of ash/slag was deposited on the furnace walls; this was done to compare the profiles with and without the effect of ash on the walls. Careful comparison shows that the boundary layer does thicken between the two ports, as expected, with some small increase in the main stream velocity (which is otherwise fairly constant) to accomodate the same flow quantity.

Figure 6.7 then gives profiles after removal of the ash, for ports 4 and 10 (lower graphs) and also for port 1 , and for the introduction cone (respectively, RH and LH graphs). These 
results are shown for two additional flow rates in addition to a repeat of the flow rate used for the experiments shown in Fig. 6.6. Superposition of the comparative graphs for port 10 in the two figures shows clearly that the boundary layer is thinner with the ash removed and, indeed, the port 10 profile without ash (bottom RH graph in Fig. 6.7) agrees almost exactly with the port 5 profile with ash (LH graph in Fig. 6.6). With such limited data, this is more indicative than substantive, but it supports the influence of ash indicated by other combustion experiments.

The top graphs in Fig. 6.7 also show the effect of cone design, with the primary and secondary air lines directed upwards towards the truncated top of the cone. The result is an inverted profile, with the air velocity lowest in the center of the cone (except where it must drop off at the walls for the boundary layer). At port 1, much of the inversion has disappeared except very'close to the walls and, particularly, at the highest flow rate used. This did not persist very far down into the furnace, however, as shown by the ports 4 and 10 profiles. Particularly at the lower flow rates, the profiles at port 4 are almost uniform.

The upward-directed jet was then compared with one of the other flow designs used, and this is shown in Fig. 6.8. In this design, the secondary air was introduced directly into the top of the cone and flowing straight down, with the primary air (normally carrying coal) introduced in a side "tee", so that this flow was introduced horizontally. The almost parabolic shape of the flow in the cone and in the ports 4 and 9 (bottom LH graph) 
are then very clear. This shows very well the extent to which the flow profile can be affected or controlled by the method of air introduction.

A final factor or major influence that was also examined in these experiments in both cold and hot flow is the effect of draft. In hot operation, the furnace acts as a chimney, with buoyancy lifting the gas stream against the pressure gradient governing the downward flow. To control the buoyancy, the furnace has to be operated with some degree of draft and, if the draft is too great, there can be substantial air inleakage that can significantly modify the flame conditions. The draft effect can be studied by itself in the cold without the interference of buoyancy and, in these cold flow experiments, the draft was substantially increased by increasing the stream flow through an induction jet at the bottom of the furnace. The results are shown in Fig. 6.6 in the bottom RH graph. This shows the almost parabolic profile also shown in the bottom LH graph for reasonably balanced draft; and it also shows the profile with increased draft. The sharply increased flow velocity near the left hand wall is clearly evident. How far this is local air inleakage and how far it is inleakage higher up, possibly at the tube bank level, is not clear from these measurements; but the overall effect, of sharply increasing the flow to generate unexpected profiles is clear.

These results do clearly show the existence of a boundary layer of up to about 1 inch in thickness, and somewhat more in some cases; one such case would appear to be when there is 
substantial ash deposited on the walls. The flow volume in this annular wall section is still relatively small, nevertheless, and the real influence on the flame is expected to be quite small.

6.1 .5 Dust cloud dispersion. The final physical parameter of major influence that was examined was the uniformity of dispersion of the dust across the furnace horizon. This was measured using the sedimentation probe. clearly, if there was substantial difference in concentration between the walls and the main flow, this could be expected to affect the flame speed quite substantially. The source of such variations could be imperfect mixing of the primary and secondary streams, with the coal being thrown out towards the walls if the cone mixing design is imperfect. It has to be said at this time that the results are not absolutely clear; there is some degree of ambiguity, as we show. Nevertheless, the uniformity shown over most of the horizontal cross-section suggests that the variations that were found are most likely due to measurement error not to poor mixing or centrifugal effects.

The results are shown in Fig. 6.9, with measurements in the cone and at ports 5 and 8 . The essential uniformity across the midsection at each level is quite clear though the slightly inverse curve in the cone (top graph) suggests that, indeed, the wall concentration may be higher than in the center stream. If true, this has rectified by port 5 except on the LHS, and then has dropped at both walls at port 8 . Interference by the wall that distorts the flow in sedimentation is a possibility, and thus distorts the real behavior. This means that the results at 
the walls have to be considered arguable, but the rest of the measurements do support the good uniformity of the concentration over the rest of the cross-section. This is an important result.

\subsection{Hot Flow Profiles}

The principal focus of the flame experiments was on the horizontal gas concentration profiles, but with particular attention given to the effects on the profiles due to other different physical conditions and, notably, the influence of draft in the hot furnace, and the influence of the air mixing behavior at entry to the cone.

6.2.1 Influence of draft. The effect of draft in cold flow has already been discussed in sec. 6.1 .4 and this extends the discussion to behavior during combustion. Under these conditions, the effect can be monitored from the influence on the combustion behavior, and notably the effect on the gas concentrations. The results are illustrated in Figs. 6.10 and 6.11. Figure 6.10 is for vertical profiles under increasing draft and Fig. 6.11 illustrates some of the relevant horizontal profiles.

Figure 6.10 shows the $\mathrm{SO} 2, \mathrm{CO} 2$, and 02 concentrations at low, medium, and high draft (top, bottom left, and bottom right graphs respectively). The rising concentration of combustion products ( $\mathrm{SO} 2$ and $\mathrm{CO} 2$ ) with time and falling oxygen, are clearly seen at low draft, and the flattening of all three curves as the draft increases is also very clear, with the $\mathrm{CO} 2$ strongly reduced in the front segment of the flame, the oxygen increased in the downstream segment, and the values increased over those obtained 
at low draft. The interpretation leaves little room for ambiguity. Inleakage is diluting the combustion products and increasing the oxygen concentration. The combustion rate may or may not increase on account of the increased oxygen: this depends jointly on the initial oxygen level -- depending on whether the oxygen is overall deficient -- and on the extent to which the temperature is also reduced by the dilution which can offset any increased reaction rate from increased oxygen concentration. From these results it is clear that accurate measurements are obtained only for the lowest feasible draft settings since it is difficult if not impossible to perfectly seal the combustion chamber.

The problem is further complicated by the existence of non-linear horizontal profiles, however, so that inleakage behavior is more complex than might be expected. This is seen from the profiles in Fig. 6.11. [In these profiles, the length of the sampling probe was designed for measurements on-axis so that in measuring the profiles, from one side of the furnace (left hand side) it was only possible to measure to just over the mid-point in the cross-section when measuring from only one side. In a few cases, measurements were made from both sides but in most cases, this was precluded on account of time. It would have been possible to design new probes able to measure across the full cross-section but the time and expense did not justify such new construction].

What is initially significant about these profiles is that, at the wall, the oxygen is low and the co2 is high. This 
can be accounted for in a number of different ways. The most probable is that the flame front is cone-shaped, as noted in all the previous discussion, so that the reaction rate must be increasing on moving from the axis to the wall at any horizon. An alternative that was considered first, however, was that, the same results would be obtained if the coal distribution was not uniform, with the coal at higher concentration near the walls. With more burning near the walls, the oxygen would be reduced correspondingly. This prior argument precipitated the investigations into the uniformity of dust dispersion already described, but the relevant measurements already discussed in sec. 6.1 .5 do not support this conclusion. Consequently, the present view is that the horizontal gas concentration profile patterns reflect the structure of the flame front.

6.2 .2 Influence of cone design and other firing conditions. For the reasons already given, associated experiments on firing condtions also focussed primarily on the influence of the mixing behavior in the cone in the initial explorations of the horizontal profile effects. These investigations were carried out prior to the dust concentration measurements and were an indirect means for examining the possibility of non-uniform mixing of the coal with the secondary air. In most of the reported experiments, the method of secondary air introduction was in paraxial flow at the apex of the cone, generally with the primary stream supplied horizontally at a "tee" just below the top of the cone as it was opening out. When it became apparent from profiles such as those in Fig. 6.11 
that the stream at the wall might be fuel rich, it was thought that this could be due to the primary stream impinging on the other side of the cone, and remaining mainly in a wall stream or wall jet (with some degree of circulation round the cone). To counter this possibility, the "tee" was moved up in one redesign, into the parallel-sided pipe supplying the secondary air so that the jet issuing into the top of the cone would be more likely to be fuel rich in the center than at the edge. This did not, in fact, have any evident effect on the flame structure and behavior (though it did evidently increase the velocity variability across the furnace), and the subsequent measurements supported the conclusion that the coal uniformity was not, therefore, the source of the flame structure problems. In other changes: the air and mixture supply lines of one inch diameter were replaced by 5/8-in to increase the velocity in the line to provide better suspension, and also to provide more intense mixing from the higher velocity; the supply line to the cone top was shortened; and the cone was rotated through 90 degrees to check for any sort of fluid coupling between the direction of inlet for the secondary line and a preferred direction determined by the tube bank. The results of these and related changes are illustrated in Figs. 6.12 to 6.14 .

When mixing uniformity was first suspected as the cause of the cone-shaped flame front, the change in supply line diameter was the first attempt to increase the uniformity by increasing the injection velocities. The results are shown in Fig. 6.12 where the top graphs show the vertical temperature and 
velocity profiles down the furnace, and the bottom graph shows the (full cross section) profiles of the gas compositions. These last show more clearly than the previous plots the full variation across the furnace. These are very similar in values and curvature to those in Fig. 6.11. The increased mixing did not evidently affect the shape or magnitude of the horizontal profiles. In subsequent experiments using a shortened cone section, in case this was producing a non-uniform fuel distribution, and then also rotating the cone, the results shown in Fig. 6.13 again are seen not to be affected by these physical construction changes. In this figure, the RH graph is for measurements made later in time on the same run compared to those in the LH graph, and the furnace conditions had also changed somewhat due to ash build-up on the walls. These results can be compared with those in Fig. 6.14, firing under fuel-rich conditions, first using the standard cone design and then using the modified introduction pipe with the coal in the straight section to focus the cloud more in the center. These results, though limited, do show some influence on the profile, with the increased slope towards the wall using the lengthened cone entry most probably due to a significantly increased velocity difference between the axis and the wall on account of the change in design. These last results were repeated in a subsequent run, with essentially the same result as shown in Fig. 6.15 though firing at substantially more fuel rich conditions.

In a final set of measurements, however, relative temperature measurements using a thermocouple in the flame were 
made, and these are shown in the RH graph of Fig. 6.15. These values are all greater than the wall at that point (of about 1100 C) so the drop towards the wall is to be expected since the TC is then seeing the thermal load of the lower wall temperatures. With this correction allowed for, the temperature variation is then much less than would have been expected from the gas profiles and, to an acceptable approximation, the temperature profile is almost flat with a value of $1250 \pm 50 \mathrm{C}$.

6.3 Summary of conclusions. The princlpal conclusions from these investigations are that the physical characteristics of the flow such as the presence of the boundary layer and the differential velocity between particles and gas are not significant, and do not seem likely to have any influence on the flame structure. Likewise, the dust dispersion across the furnace horizontal would appear to be essentially uniform or reasonably so, with only some concern about the concentrations close to the walls where accurate measurement is difficult in any event. The precise method of introducing the air into the top of the cone does have rather more influence, with the original design of the co-axial, upward-directed jets showing an inverted velocity profile near the top of the cone, but this flattened out substantially, and was near uniform at the tube bank. This original design would appear to provide the most uniform flow pattern for the intended purpose. By contrast, the axial flow into the top of the cone is much poorer in that respect, with almost parabolic flow in the cone and at the top of the furnace. This, by itself, does not appear to be the source of the two-dimensional cone-shaped 
flames, but the measurements do indicate that this profile accentuates any two-dimensionality in the flame that occurs for other reasons. 
Table 6.1:Calculated Entry Lengths and Boundary Layer Thicknesses for Different Flow conditions in the Experimental Furnace

Flow Rate Reynold's
(scfm)

(I) COLD FLOW CONDITIONS $(T=300 \mathrm{~K})$

$\begin{array}{rrrr}11.4 & 2173 & 22 & 0.767 \\ 25.7 & 4898 & 49 & 0.340 \\ 64.2 & 12216 & 121 & 0.136\end{array}$

(II) HOT FLOW CONDITIONS $(T=1500 \mathrm{~K})$

$\begin{array}{rrrr}11.4 & 789 & 8 & 2.111 \\ 25.7 & 1780 & 18 & 0.936 \\ 64.2 & 4439 & 44 & 0.375\end{array}$

Note: The (cold) flow rates are those at which gas velocity measurements were carried out. 


\section{BOUNDARY LAYER CALCULATIONS}

BLASIUS B.L. THICKNESS (T-20 C)

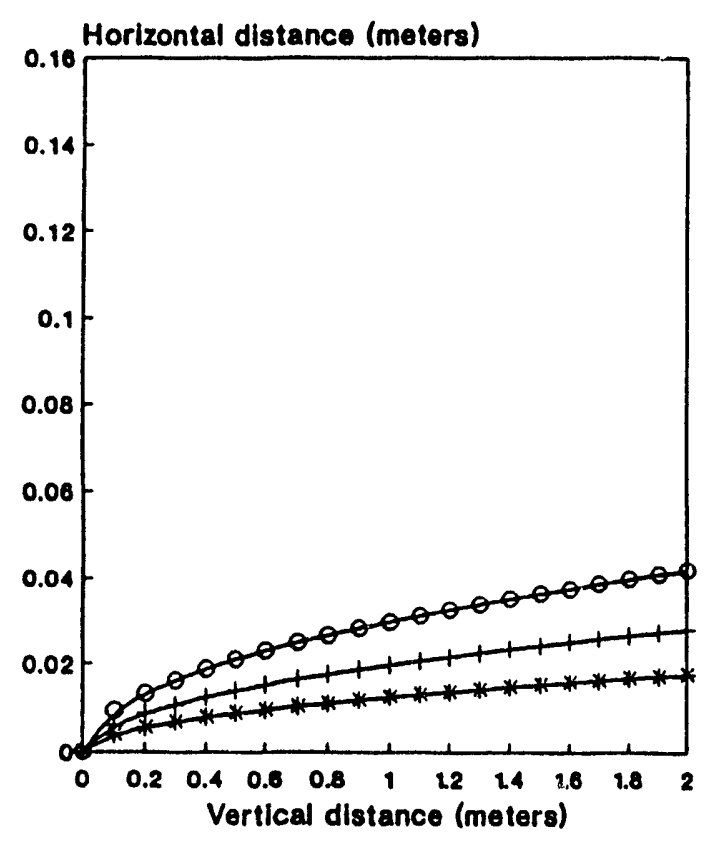

$011.4801 m \cdot 25.7801 m \cdot 64.28 \mathrm{ctm}$
BLASIUS B.L. THICKNESS $(T=1100 \mathrm{C})$

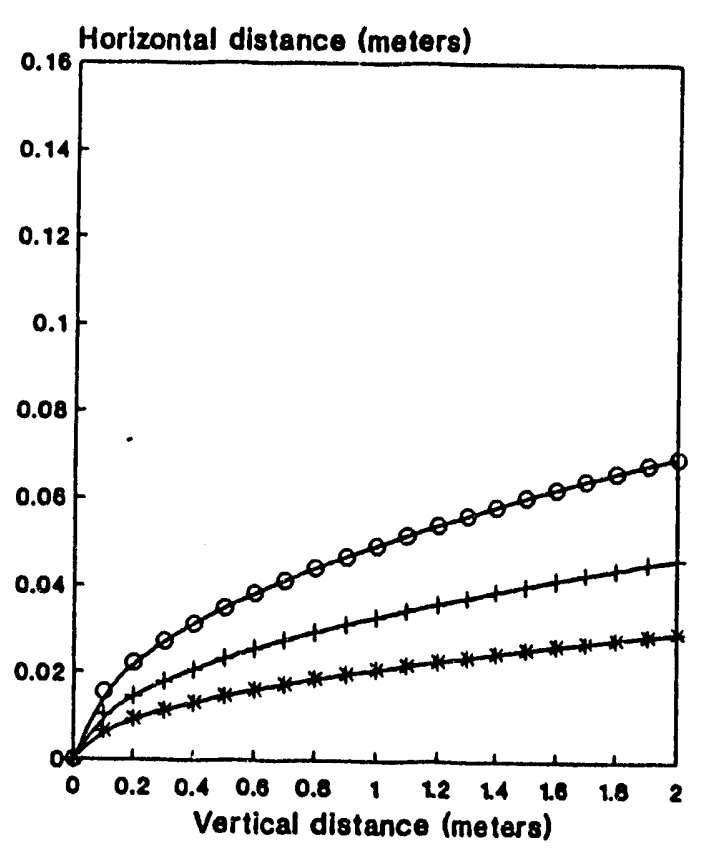

- $11.48 \mathrm{cfm}+25.780 \mathrm{fm} \cdot 64.28 \mathrm{ctm}$

FIG. 6.1 


\section{CALUULATED PARTICLE VELOCITIES}

PA:TICLE VELOCITIES $(T=20 \mathrm{C})$

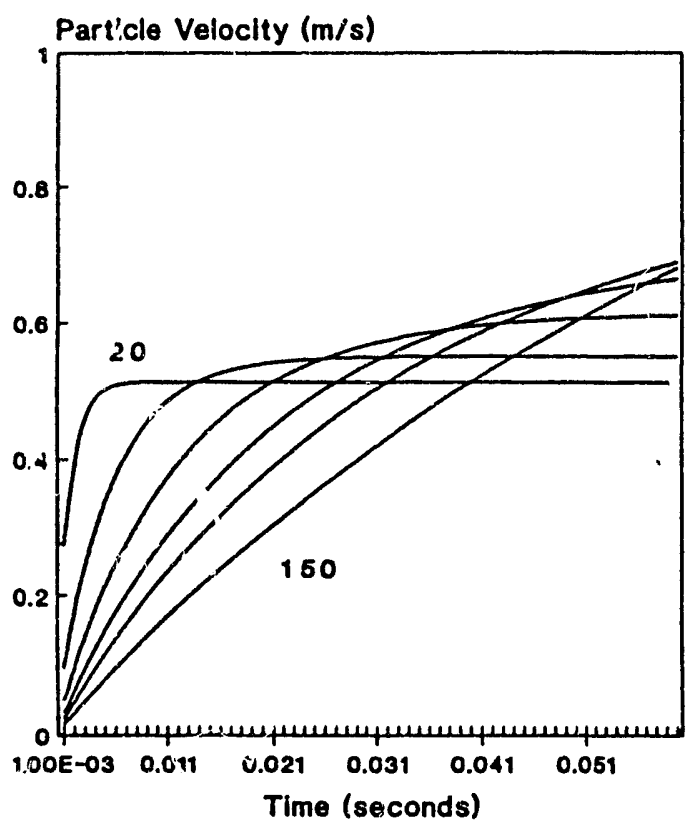

PARTICLE VELOCITIES $(T=1200 \mathrm{C})$

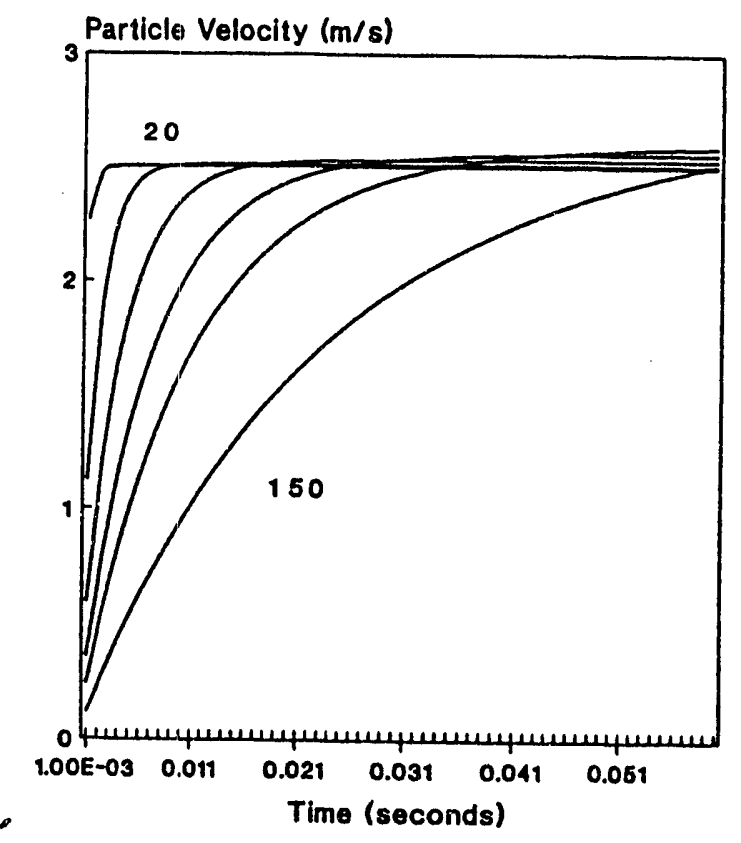

FIG. 6.2 


\section{MEASURED PARTICLE VELOCITIES}

TAC 20 micro sec, DISC 200

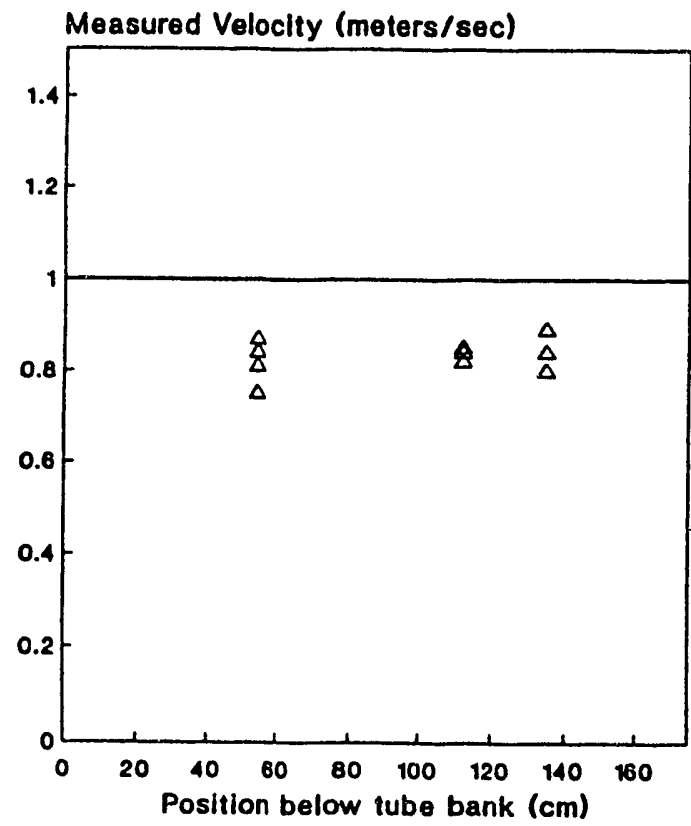

TAC 20 micro sec, DISC 200

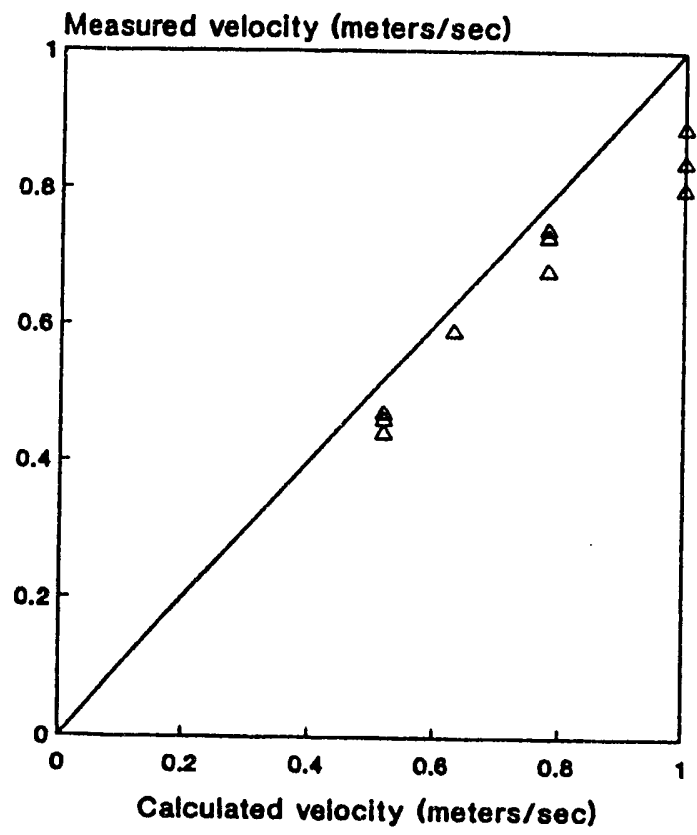

FIG. 6.3 


\section{MEASURED PARTICLE VELOCITIES}

TAC 50 micro sec, DISC 150

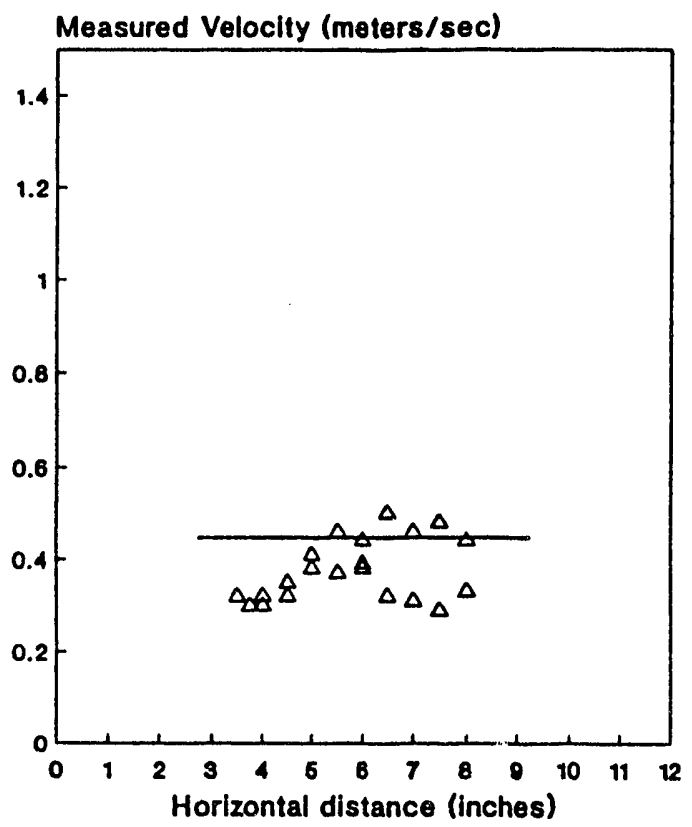

TAC 20 micro sec, DISC 80-200

TAC 20 micro sec, DISC 100-180
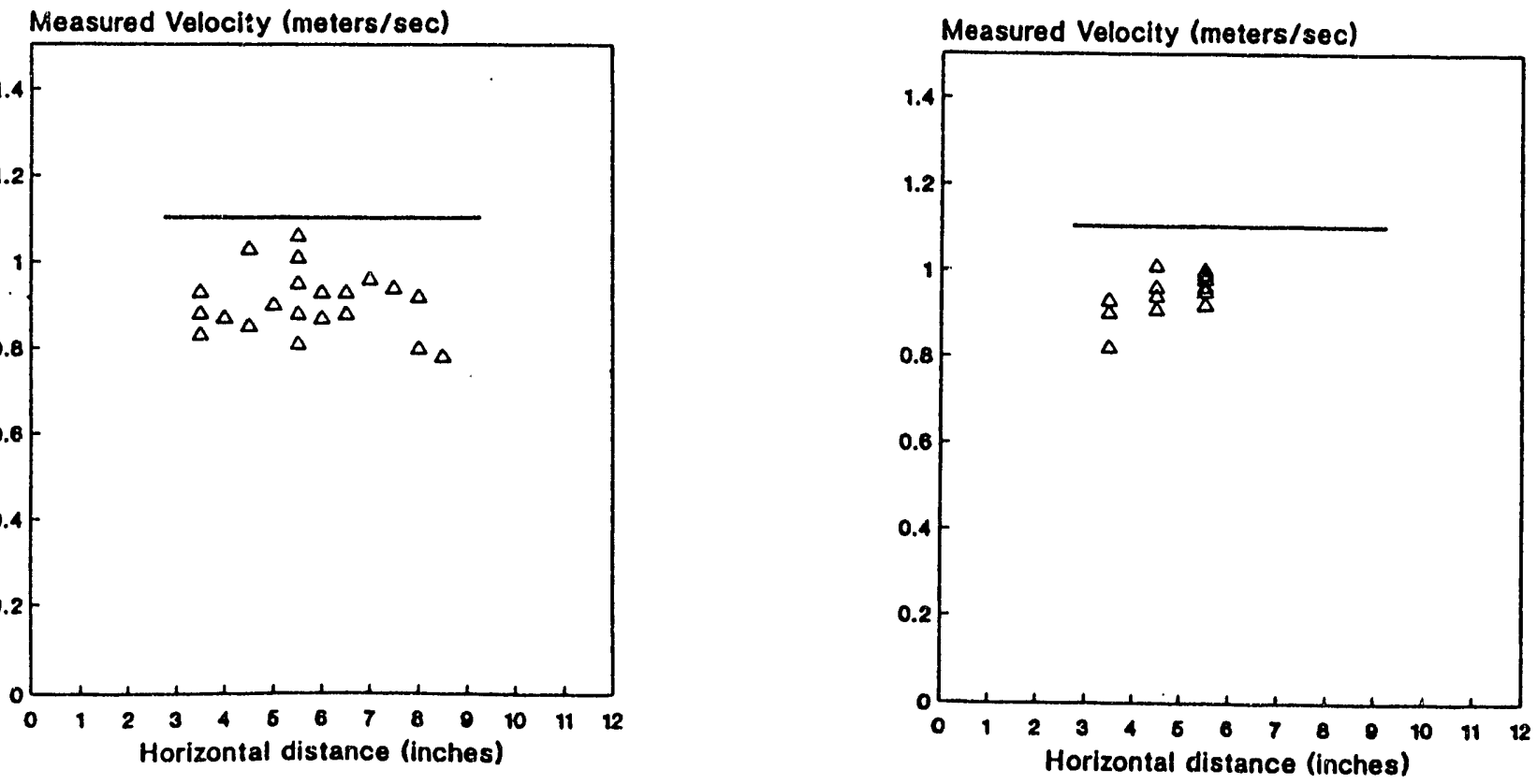

FIG. 6.4 


\section{MEASURED PARTICLE VELOCITIES}

TAC 50 micro sec, DISC 100-300

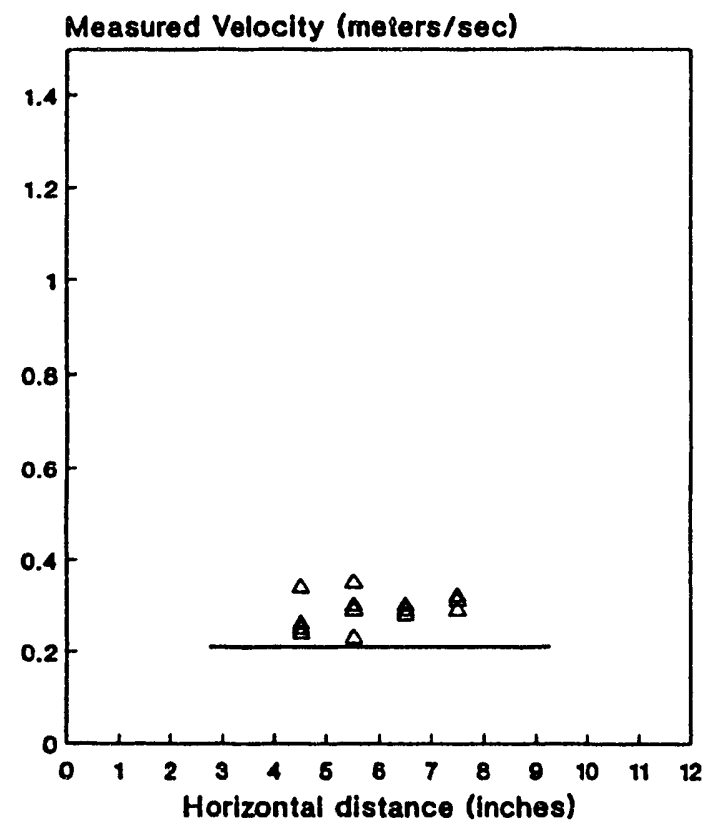

TAC 50 micro sec, DISC 140-300

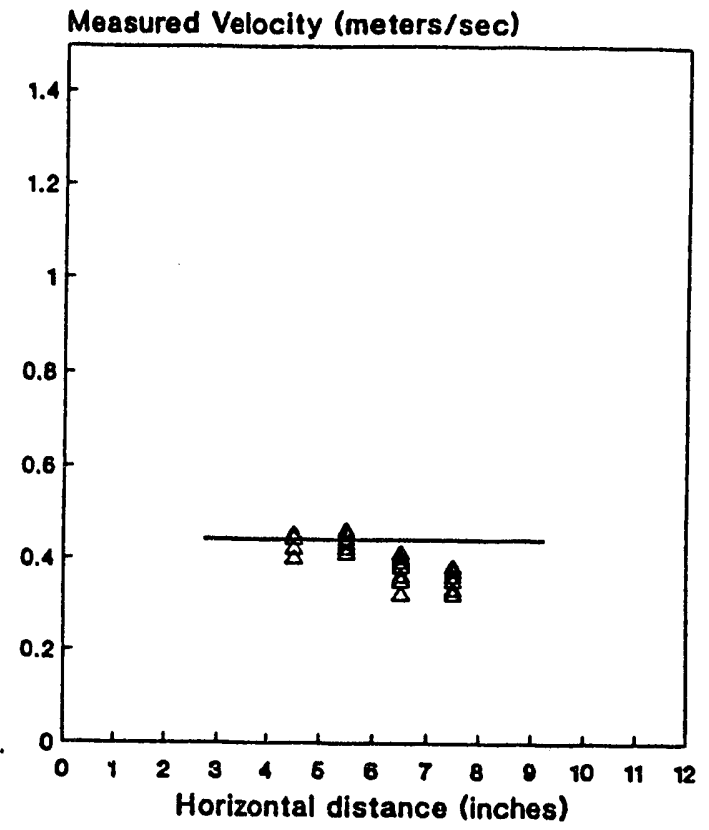

TAC 20 micro sec, DISC 100-300

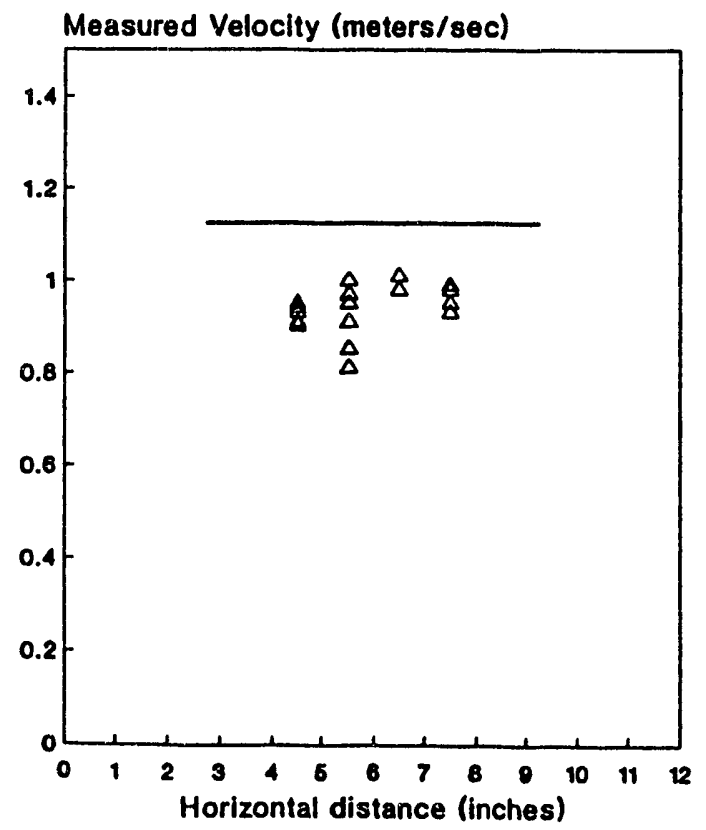

FIG. 6.5 


\section{GAS VELOCITY MEASUREMENTS}

PORT 5

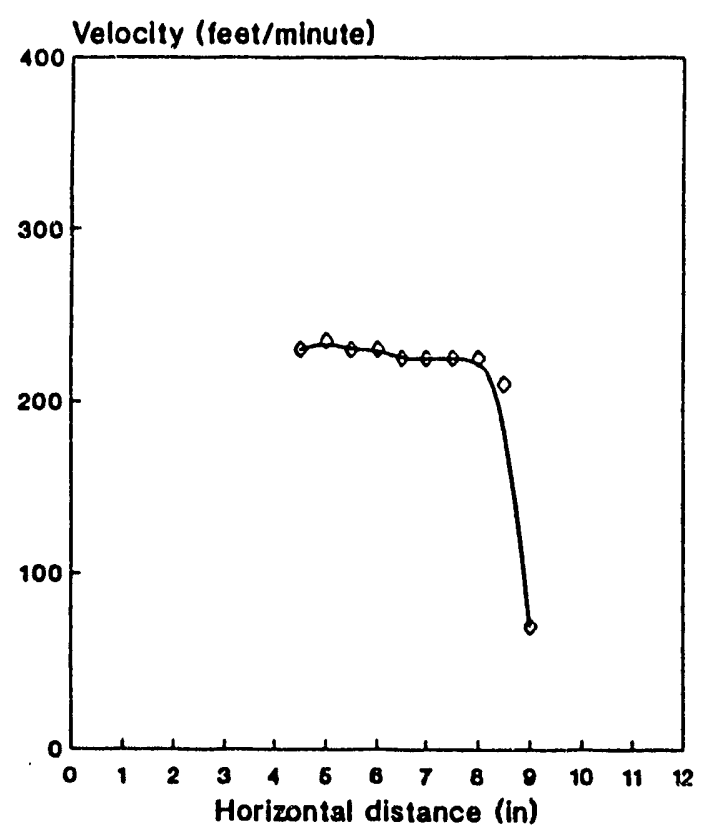

PORT 10

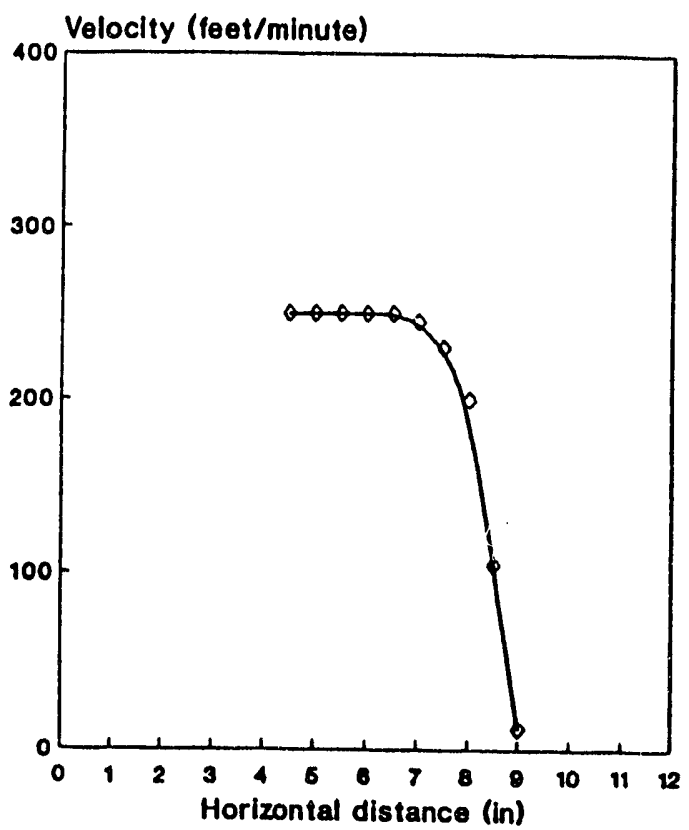

FIG. 6.6 


\section{GAS VELOCITY MEASUREMENTS}

\section{INTRODUCTION CONE}

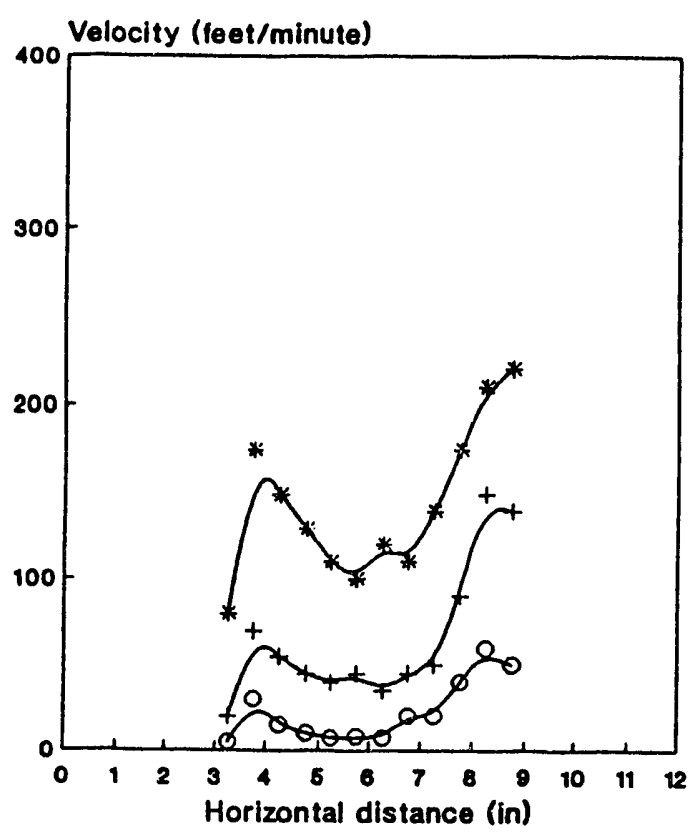

$011.48 \mathrm{cfm}+25.7 \mathrm{scm} \cdot 64.28 \mathrm{cfm}$

PORT 4

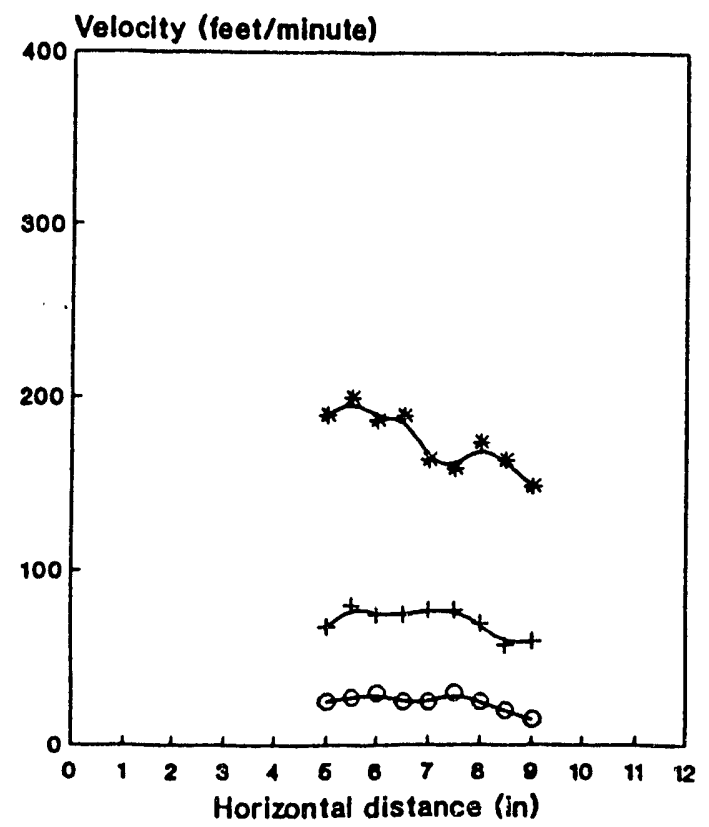

$011.48 \mathrm{cfm} \cdot 25.78 \mathrm{cfm} \cdot 64.28 \mathrm{cfm}$
PORT 1

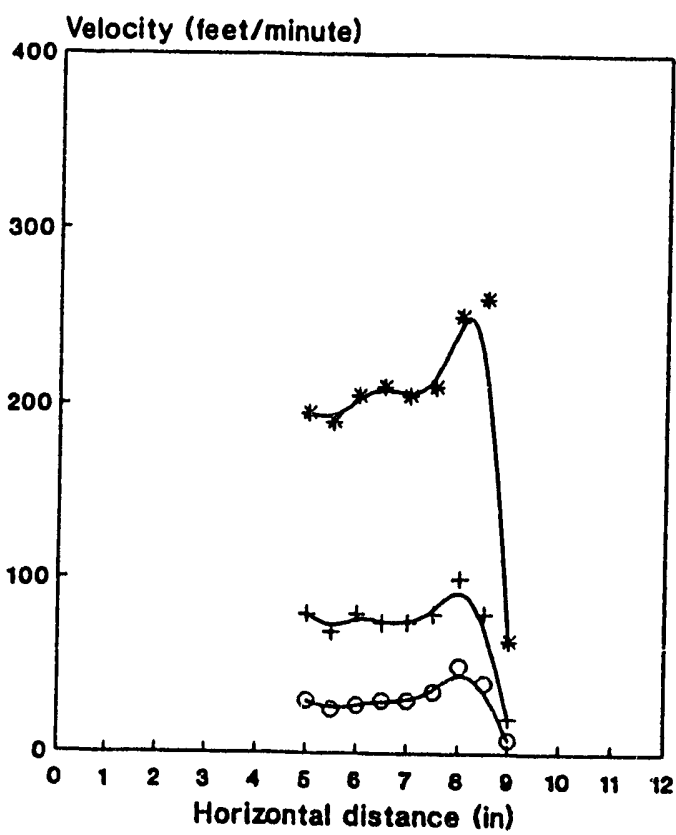

$011.48 \mathrm{ctm} \cdot 25.78 \mathrm{clm} \cdot 64.28 \mathrm{clm}$

PORT 10

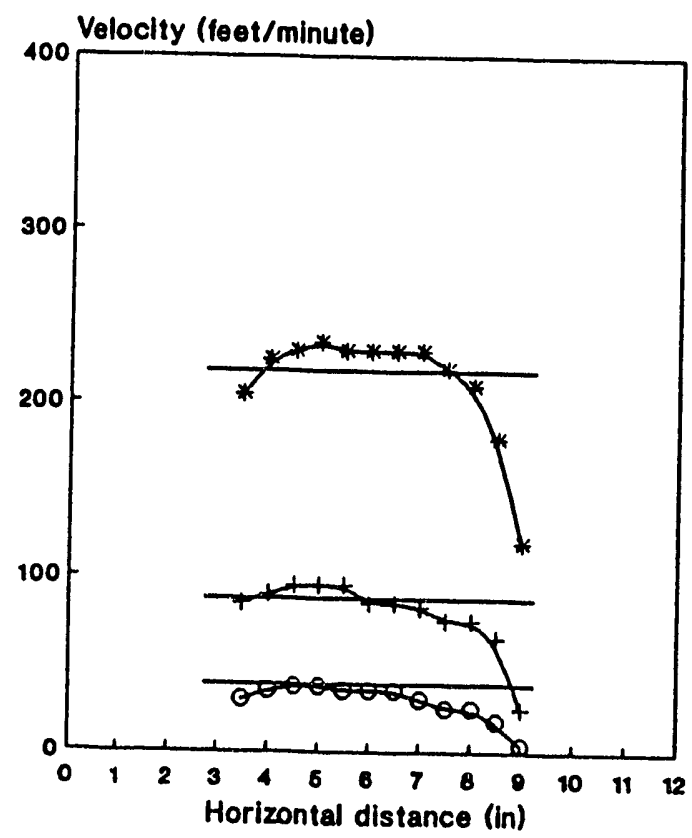

$011.48 \mathrm{ctm} \cdot 25.7 \mathrm{sctm} \cdot 64.28 \mathrm{cfm}$

FIG. 6.7 


\section{GAS VELOCITY MEASUREMENTS}

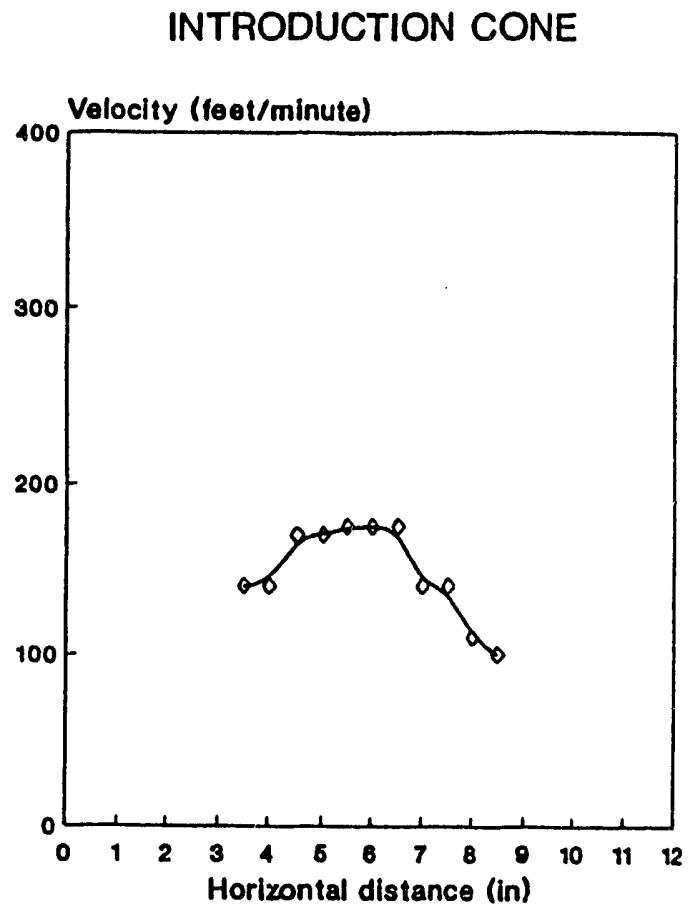

PORTS 9 AND 4

PORT 4
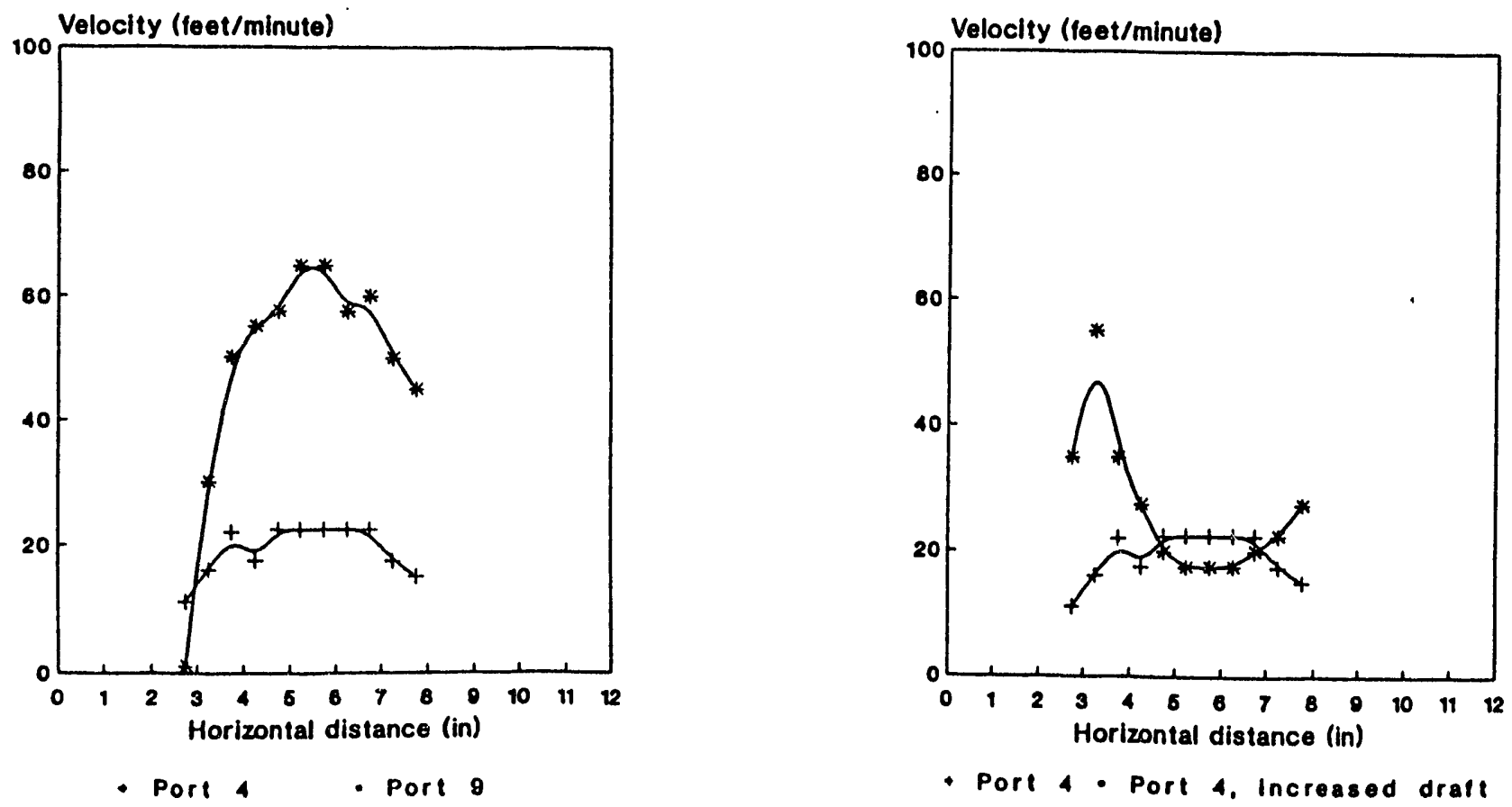

FIG. 6.8 


\section{DUST CLOUD UNIFORMITY TESTS}

\section{INTRODUCTION CONE}

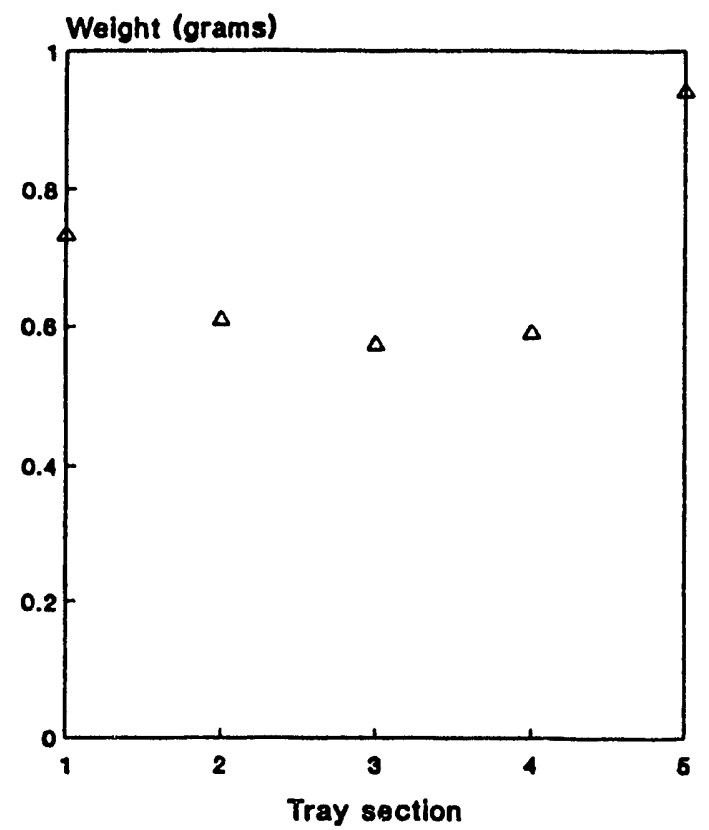

PORT 5

PORT 8
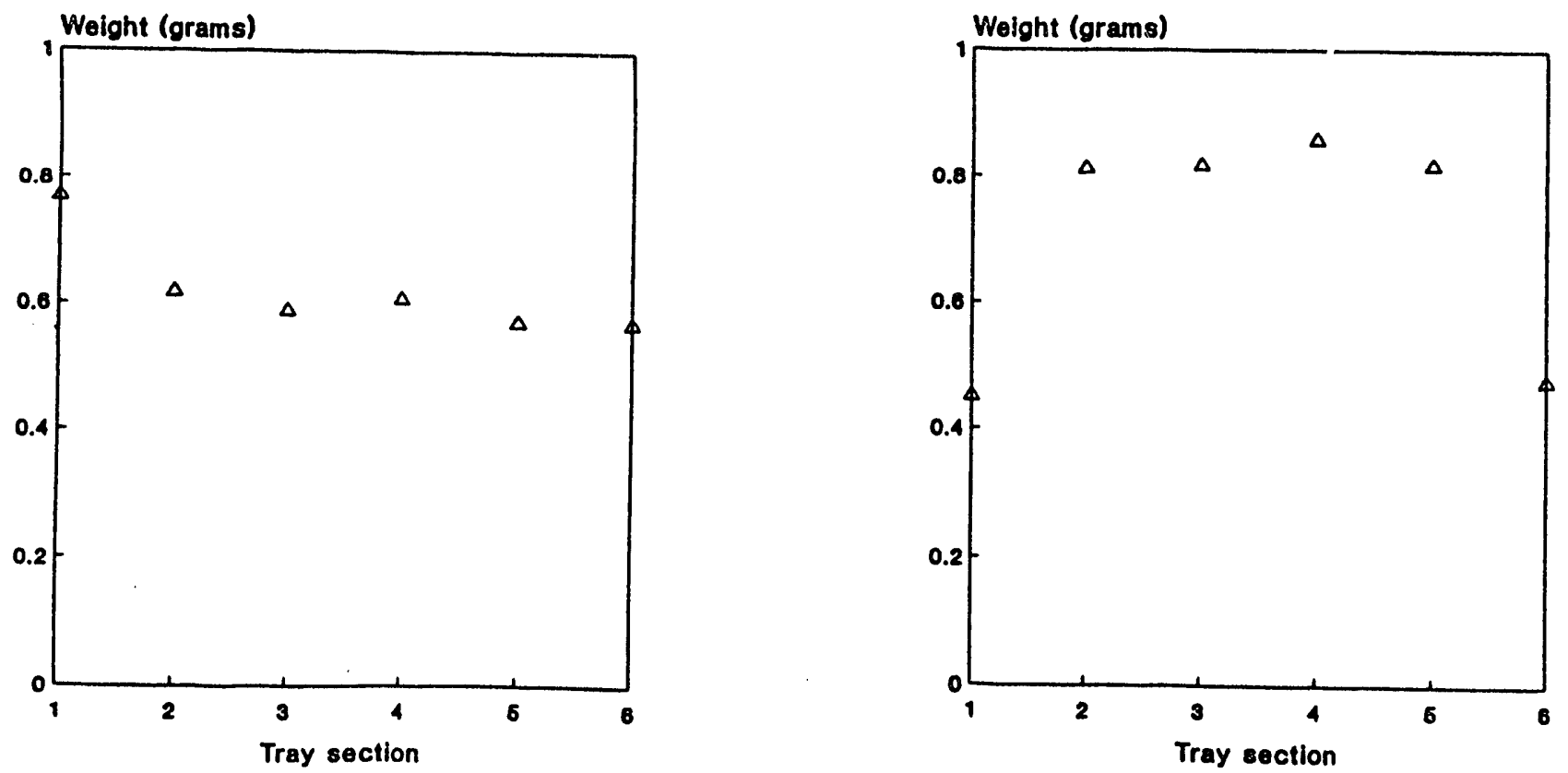

FIG. 6.9 
Run: $\quad 10-22-91$

Firing Conditions:
Coal 6

$10.6 \mathrm{lb} / \mathrm{hr}$
$E X O=-.7 \%$ $21.1 \mathrm{scfm}$

\section{LOW DRAFT}

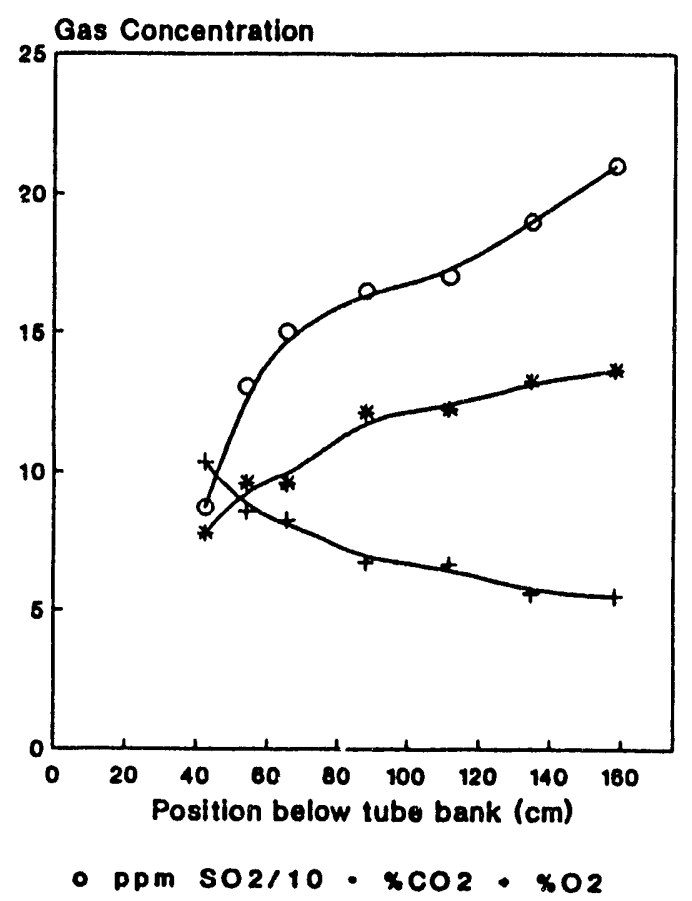

SLIGHTLY HIGHER DRAFT

HIGH DRAFT
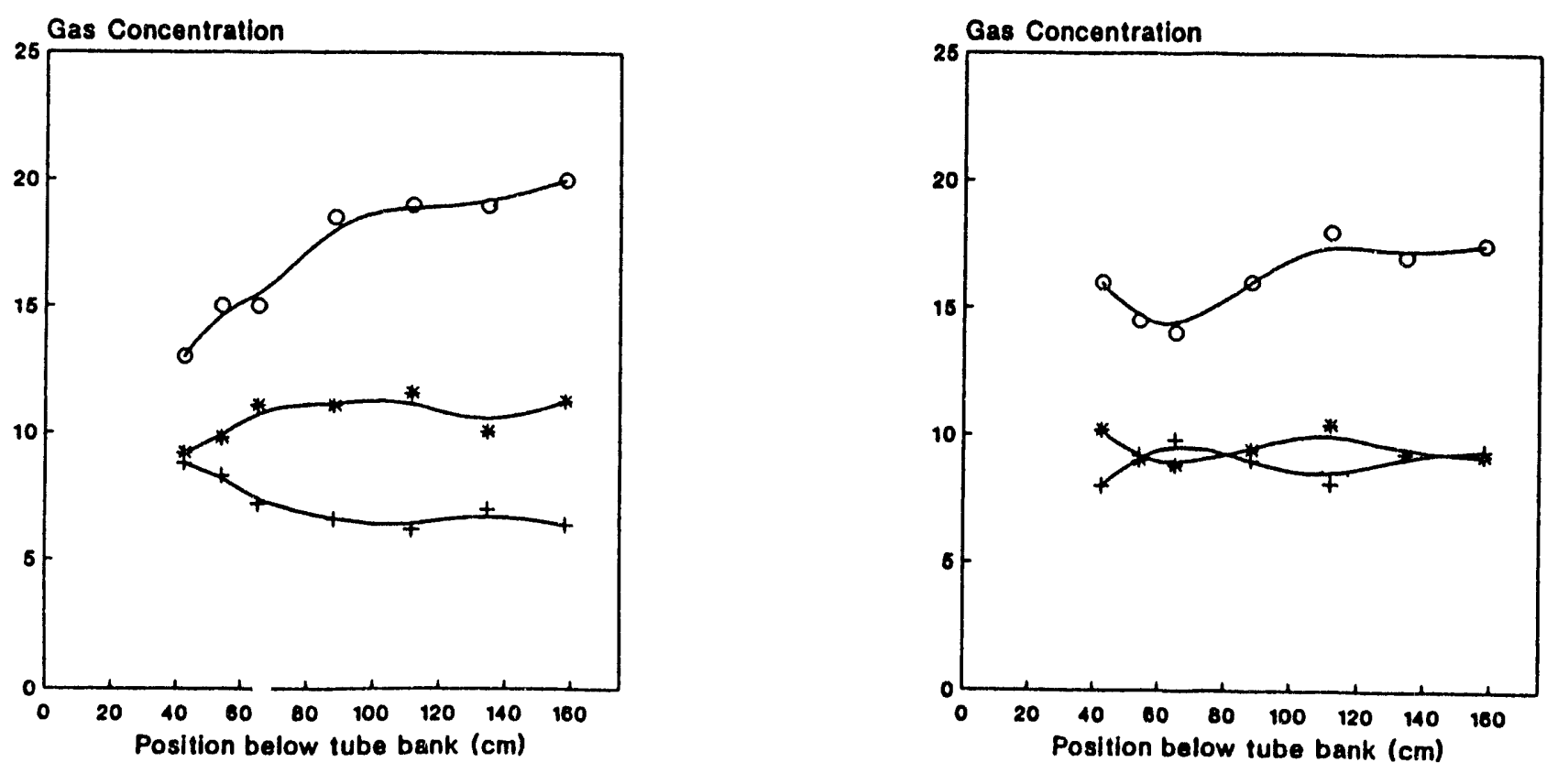

- Ppm SO2/10 *x $\mathrm{CO}_{2} \cdot \mathrm{xO}_{2}$

FIG. 6.10 
Run: 10-22-91 Firing Conditions:

HORIZONTAL PROFILE AT PORT 5

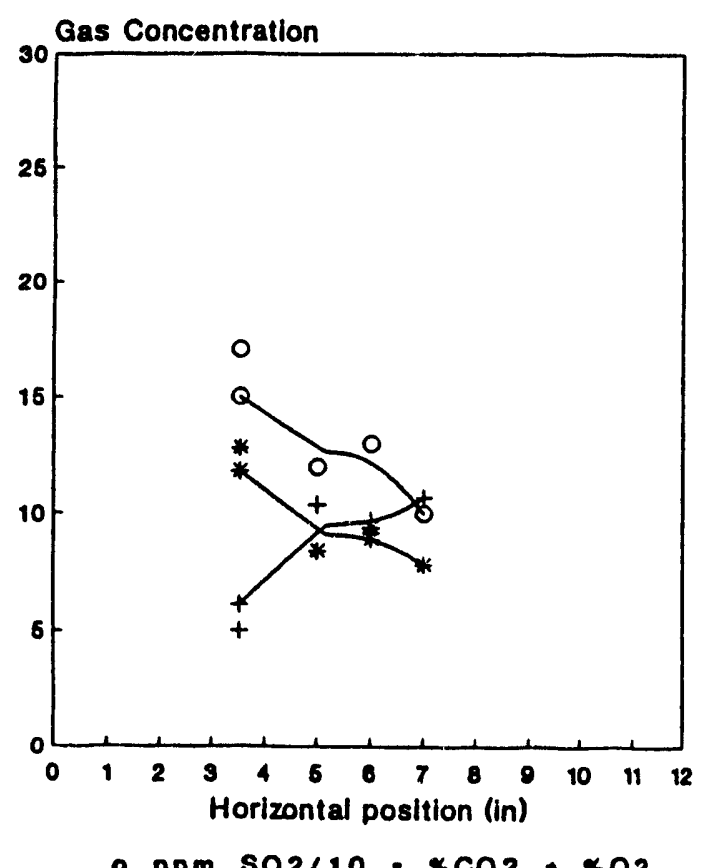

Coal 6 $10.6 \mathrm{lb} / \mathrm{hr} \quad 21.1 \mathrm{scfm}$
HORIZONTAL PROFILE AT PORT 8

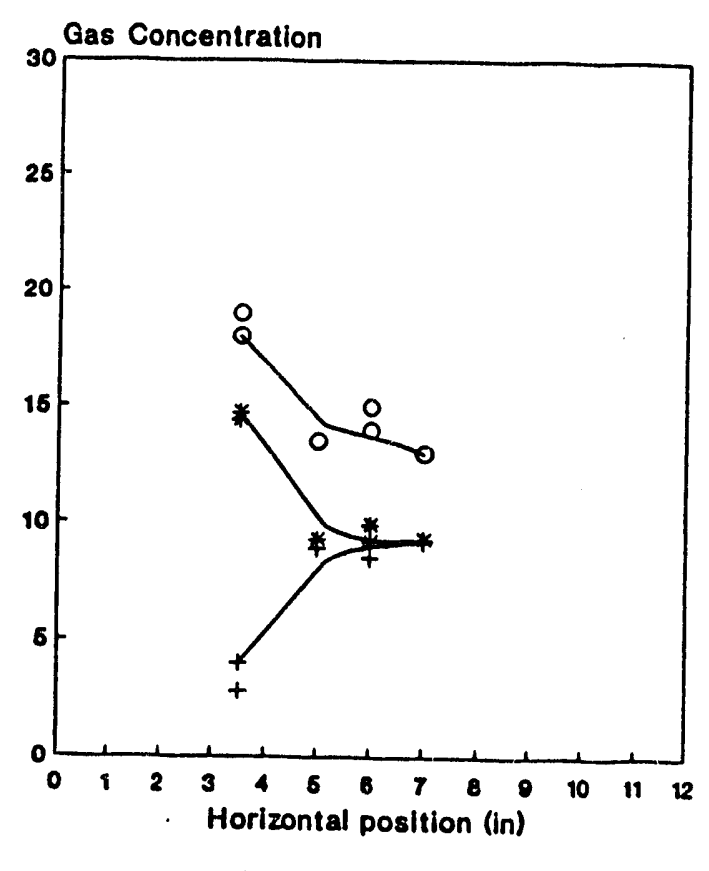

- ppm s02/10.xc02 * xO2

FIG. 6.11 
Run: 11-01-91

Firing Conditions:

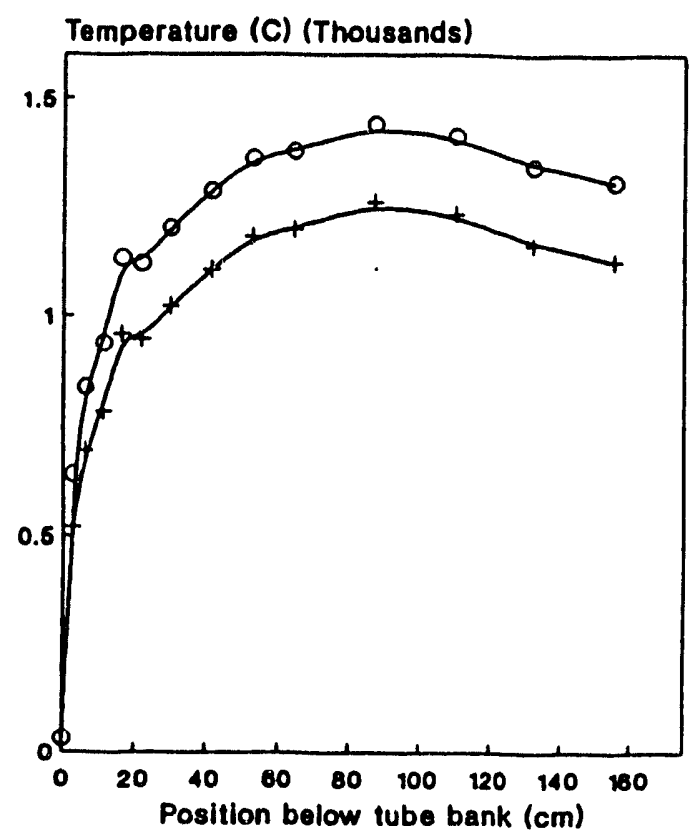

- Wall $T$ - Flame $T$ o Calculated
Coal $5 \quad E X O=-10.1 \%$ $11.6 \mathrm{lb} / \mathrm{hr} \quad 23.1 \mathrm{scfm}$

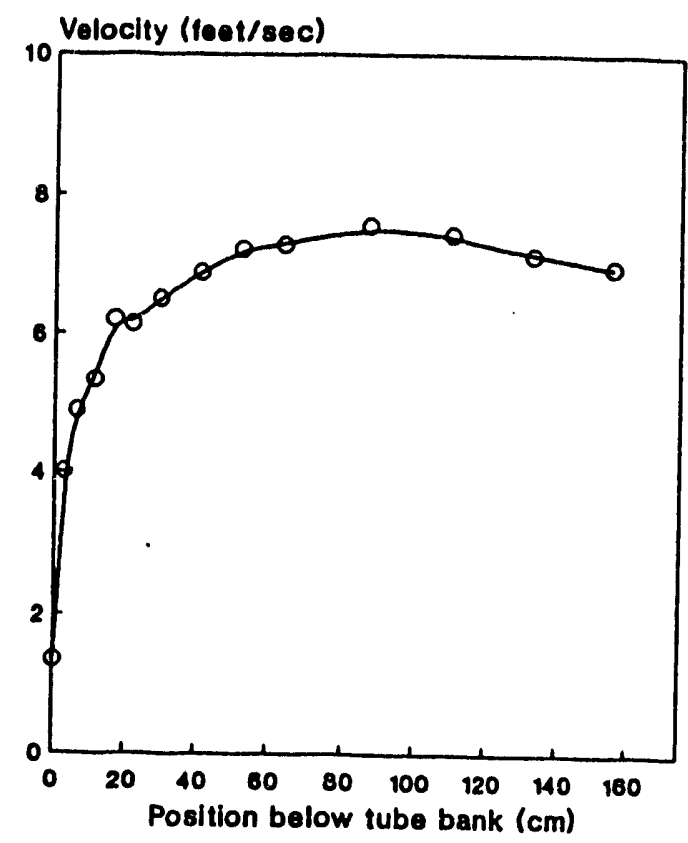

PORT 8, after replacement of air lines with smaller lines

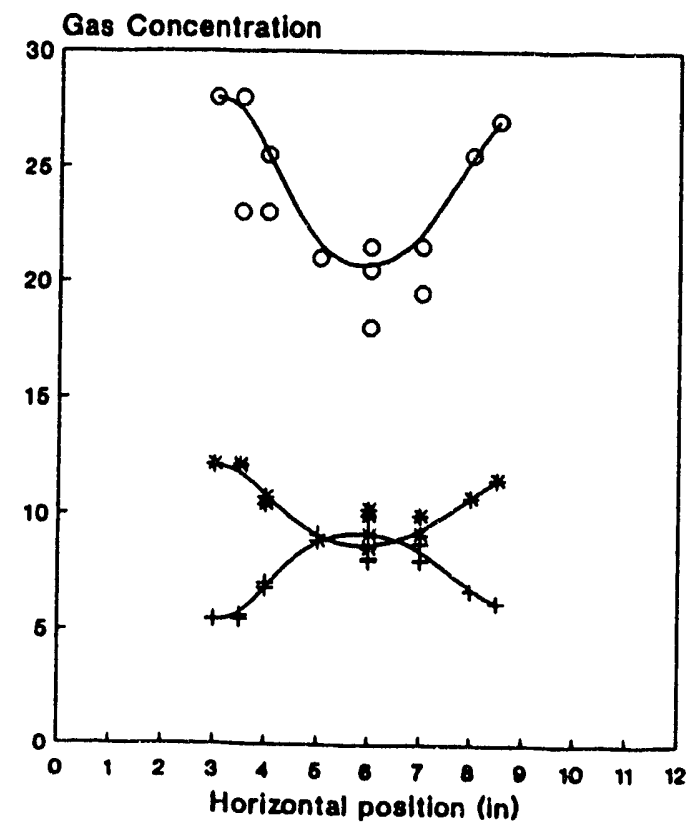

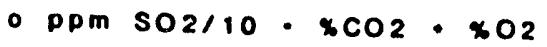

FIG. 6.12 
Run: 11-05-91 Firing Conditions:

PORT 8, shortened cone

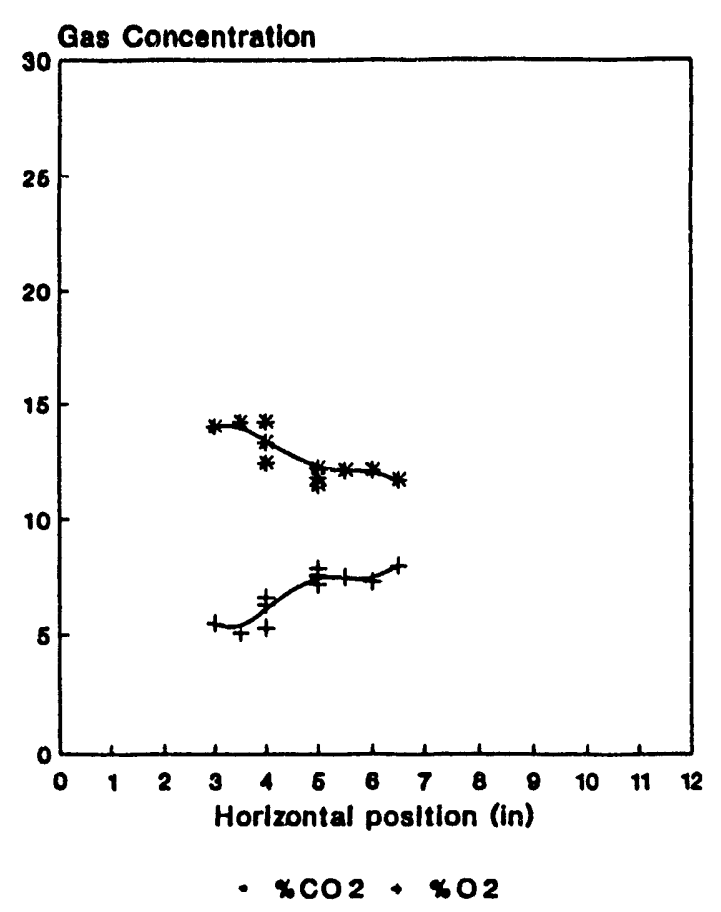

Coal $5 \quad E X o=-6.6 \%$ $11.6 \mathrm{lb} / \mathrm{hr} 23.3 \mathrm{scfm}$
PORT 8, cone rotated 90 degrees, ash build-up on walls

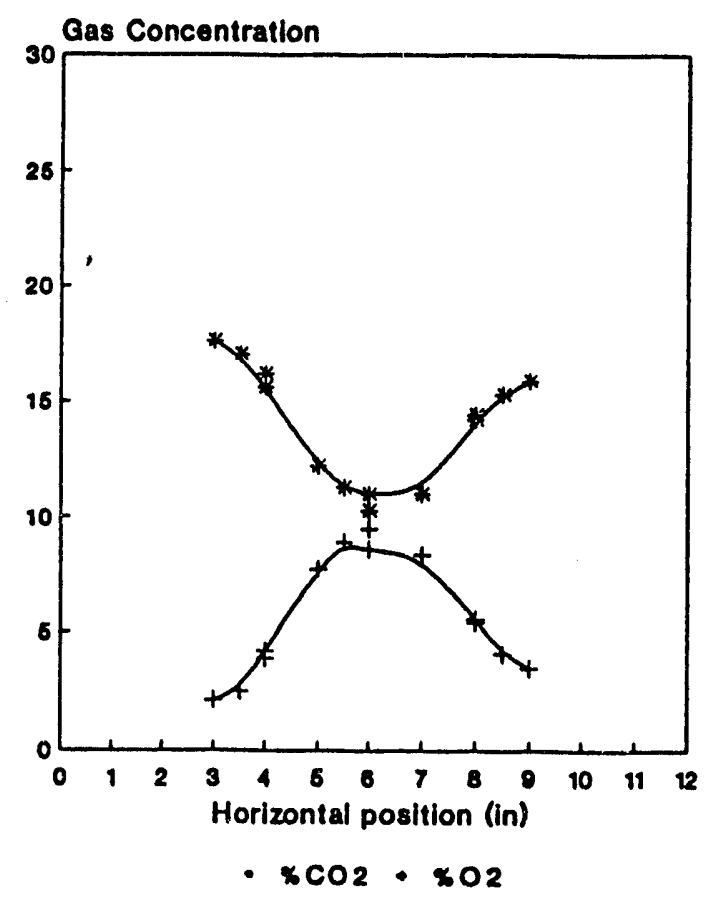

FIG. 6.13 


\section{Run: $\quad 11-07-91 \quad$ Coal $5 \quad$ EXo $=-6.0 \%$ Firing Conditions: $\quad 11.6 \mathrm{lb} / \mathrm{hr} \quad 23.5 \mathrm{scfm}$}

PORT 8, regular cone, furnace walls clean

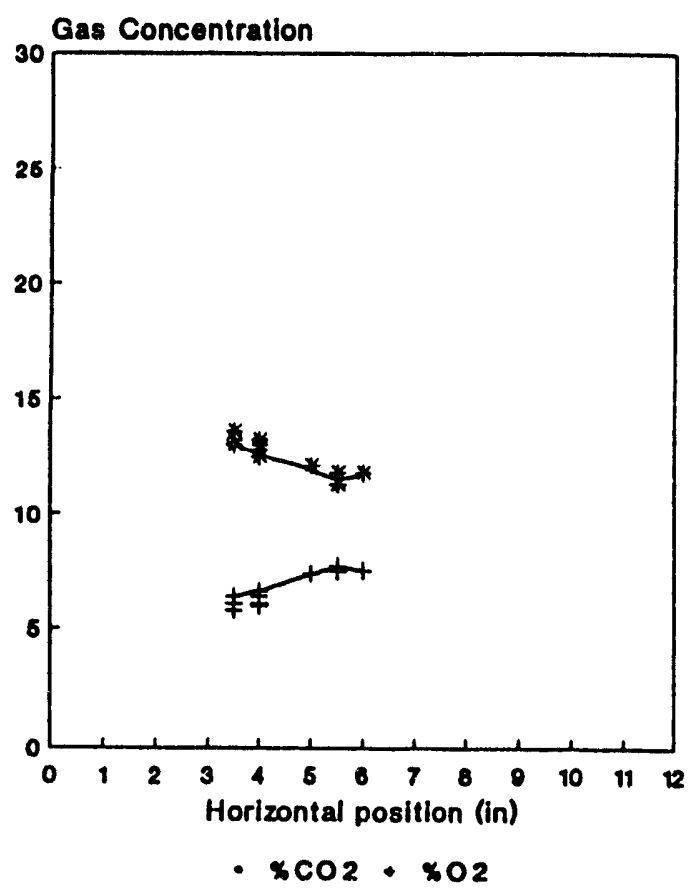

PORT, 8, lengthened cone, some ash build-up on walls

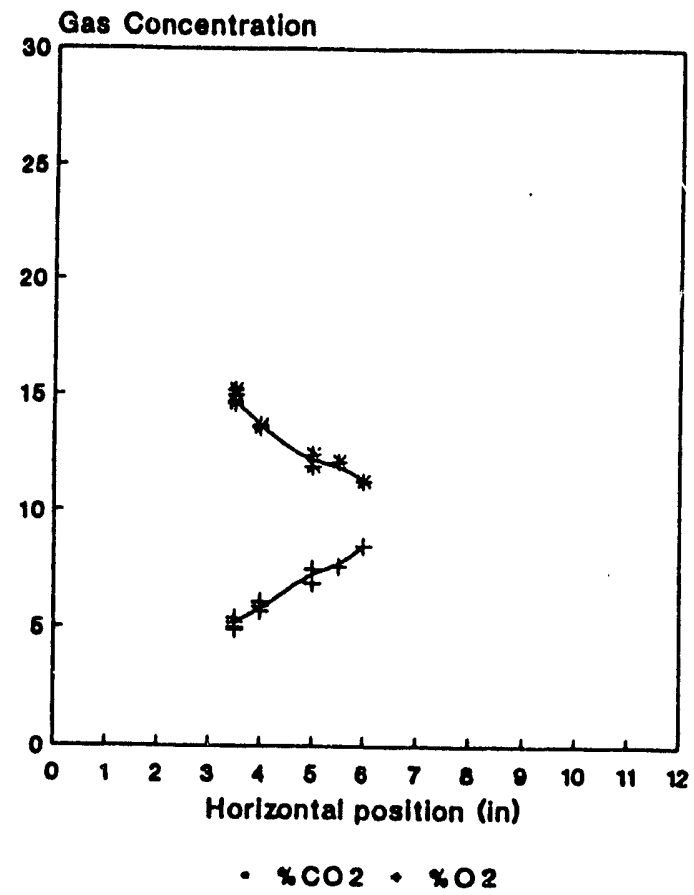

FIG. 6.14 


\section{Run: $11-12-91 \quad$ Mixture $\quad E X O=-15.5 \%$ Firing Conditions: $\quad 11.6 \mathrm{lb} / \mathrm{hr} \quad 21.1 \mathrm{scfm}$}

PORT 8, lengthened cone,

ash build-up on walls

HORIZONTAL TEMP. PROFILE
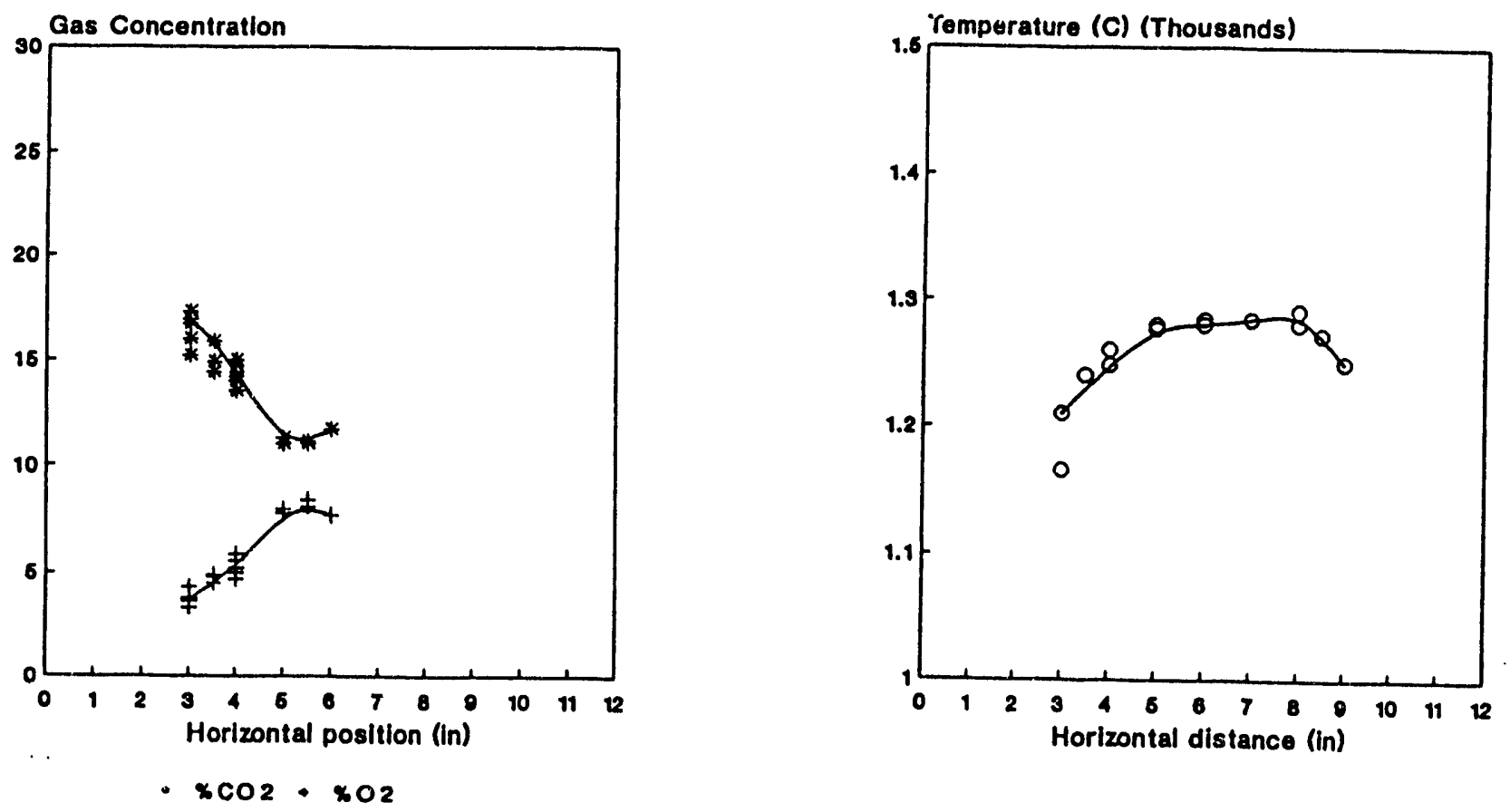

FIG. 6.15 


\section{RESULTS IV: FIAME STRUCTURE INVESTIGATIONS}

The flame profile results obtained in this investigation are illustrated in Figs. 7.1 to 7.44 . These are results from 19 separate runs using all the different coals listed, and each run is identified by date in mm-dd-yy format and arranged in timesequence of execution. The coal used in each case is identified by number listed on the figure. The graphs are variously: size distributions presented as Rosin-Rammler plots; vertical profiles of temperature, gas concentration, VM content, and reaction efficiency, mostly in Figs. 7.1 to $7.22 ;$ and horizontal profiles in Figs 7.23 to 7.44 . There is major commonality in these profiles, wit? perturbations on that commonality due to different firing and furnace conditions. In the following sections, which compare similar profiles for the different runs, this commonality is discussed along with the results which show evidence of the alternate flame structure, as discussed earlier, and also results which show the inportance of the proper sampling procedures and draft control:

This sequence of experiments was carried out in two Series (I and II) with a time separation between them, as will be apparent from examination of the dates on each graph. This came about as a consequence of discovering the evident problem of the non-linear flame structure during execution of the series I experiments (from January 1991 through September 1991; Figs. 7.1 to 7.22). This was followed by a series of exploratory and redesign experiments, largeiy though not totally described in sec. 
6 though these had some overlap with the series I experiments. These re-design experiments resolved a number of ancillary problems as already discussed but not the primary one of accounting for the cone-shaped flames. From December 1991 through February 1992, the Series II experiments were carried out with the target of resolving the flame shape problem that by that time was thought to be most likely a matter of firing and ignition conditions. The flame shape problem is still unresolved but, in our view, we were converging on the answer when it become necessary to terminate the experiments for lack of time. The findings on this are described in the material following.

7.1 General character of the Flames. In visual observation of the flames at different locations down the furnace, there is some variation of flame character, but the first-order qualitative characteristics of all the flames are essentially common. The light emission that characterizes the process of combustion starts near the top of the furnace, just below the tube bank in most cases, and is generally quite bright due to the (obvious) emission and combustion of volatiles. The leading edge of this emitting zone can be termed the flame front. The onset of ignition, defined here as the location of the evident flame front, is generally 5 to $10 \mathrm{~cm}$ below the tube bank, with this determined primarily by the type of coal, the coal PSD, the firing rate, and stoichiometry. Depending on the type of coal, the volatiles combustion region typically continues for about 10 cm down through the flame and, below that, the combustion is sustained by continued reaction of the char. In most though not 
quite all of the flames generated in this study, the passage of discrete coal particles down through the flame is evident to the eye as motion of bright lines and, moreover, so also are fluctuations in the brightness of the flame due to perturbations in the feed rate.

The shape of the flame front is not easily discerned just by visual observation. If the flame is not flat then the line of sight at the level of the flame front is through the edge of the flame where it must attach at both walls, and so observation near the central part of the furnace to determine whether or not ignition is occuring there (ignition in the central region for a flat flame, no ignition in the central region for a conical flame) is nearly impossible except in extreme cases. It was largely for that reason that it was necessary to rely on the horizontal profiles through the flame to be able to say with some certainty that the flame structure was not what was expected, and it was the concentration measurements that first indicated that there might be a problem.

In contrast, qualitative observations of the flames developed in prior work showed some evident differences. Notably, the flame in the region of the volatiles combustion was generally so bright that it could not be observed without dark glasses. In the char combustion region, the combuston gases took on the more uniform appearance of gas combustion, and the individual particle tracks were rarely observed except at the top of the flame approaching the flame front. In particular, at the flame front, there was a characteristic qualitative difference in 
that the obvious particle or cluster tracks at the flame front were generally straight down in almost parallel lines in the prior work whereas, in the current experiments, close observation of these tracks showed possible backmix flows, most probably due to local buoyancy (specifically excluded in prior work by helium tracer experiments). Details of the measurements are provided in the sections following.

7.2 Temperature profile. Wall temperatures were measured in all experiments. Flame (particle) temperatures were measured in some experiments, mostly using 2-color pyrometry; in a limited number of cases, some relative horizontal temperature profiles were obtained using thermocouples. Since the wall and flame temperatures generally correlate quite closely then, when only wall temperatures are available, flame temperatures can be estimated quite accurately using two-temperature correlations.

Characteristically, the temperature profiles are as shown, typically, in Fig. 7.1, rising rapidly from ambient in the first $10 \mathrm{~cm}$ or so, then rising with diminishing gradient to a maximum value, and then falling to the end of the flame. The flames then differ quite characteristically in two respects: the location of the peak temperature, which can vary from 10 to 100 cm downstream from entry, and the peak temperature, which can vary from $1250 \mathrm{C}$ to over $1500 \mathrm{C}$. Characteristically, the stoichiometry tends to govern the peak temperature, and the firing rate governs the peak location. In particular, the flame temperature in PC flames generally peaks between $-25 \%$ and $-30 \%$ excess air. Coal type and PSD can also play a part, however. 
Wall Temperature. Because of the correlation between wall and flame temperatures, the characteristics and characteristic shape of the wall temperatures are governed essentially by the same factors that determine the similar flame characteristics. The peak wall temperatures range from approximately 1250 to $1450 \mathrm{C}$ (with the exception of run 1-17-91 with a peak temperature of $1135 \mathrm{C})$ at a distance of 70 to $100 \mathrm{~cm}$ below the water cooled tube bank. The variations in the wall temperature profiles are due to the different stoichiometries, firing rates, and furnace conditions for each run.

Run 1-17-91 (coal 13 at a rate of $13.91 \mathrm{~b} / \mathrm{hr}$ ) and run 119-91 (coal 13 at a rate of $16.3 \mathrm{lb} / \mathrm{hr}$ ) have peak wall temperatures of 1135 and 1255 c respectively, with this temperature difference being due primarily, in these two cases, to the difference in stoichiometry (excess air: at $16 \%$ and $-7 \%$ respectively) with differences in firing rate of lesser importance.

In contrast, runs 8-6-91 and 8-13-91 have nearly identical firing rates and excess air levels, and the fuels for these runs, coal 1 and coal 3, have almost the same heating values; however, run 8-6-91 has the lower temperature profile, and this is attributed to the higher draft setting for this run which is expected to have been responsible for some air inleakage. The same is probably true for runs 8-19-91 and 9-4-91 which also show a difference in temperature profile as well as peak temperature, at 1390 and $1260 \mathrm{C}$ respectively, although the heating values of the coals are similar and run 9-4-91 had a fuel 
rate of $14.6 \mathrm{lb} / \mathrm{hr}(\mathrm{coal} 4)$ at -18 excess air compared to 12 lb/hr (coal 3) at $5 \%$ excess air for run 8-19-91. The higher peak temperature for run 8-19-91 at the higher excess air is most likely to the lower draft setting for that run.

Another shaping characteristic is seen in the series II experiments where there were several runs at substantially fuelrich conditions. This included runs 12-19-92, 12-21-92, 1-8-92, 1-14-92, and 2-14-92 where the excess air levels ranged mostly from $-20 \%$ to $-30 \%$, with $-52 \%$ in the last run. Under these conditions, the peak temperature moved upstream, also giving the temperature profile a much flatter look, comparing, for example, runs 12-19-92 and 12-21-91 at $-29 \%$ and $-2 \%$ excess air respectively. These flatter temperature profiles are much closer to those observed in previous work.

Finally, another attemperating factor is the presence of limestone, as shown in the first and second runs on 9-9-91, both with coal 9. The excess air drops from $-1 \%$ to $-28 \%$ (with an increase in firing rate) which normally could be expected to increase the peak flame temperature. However, the peak temperatures were $1320 \mathrm{C}$ (at $15.6 \mathrm{lb} / \mathrm{hr}$ ) and $1250 \mathrm{C}$ (at 18.1 $\mathrm{lb} / \mathrm{hr}$ ) respectively, and the drop is attributed at this time to the limestone loading, which may also have affected the radiation field on which the flame stabilization depends.

In general, differences in (wall) temperature profiles are due primarily to fuel and air rates (stoichiometry and firing rates) and draft control (which causes air inleakage if the draft is too high, thus increasing the actual excess air level); but 
other factors such as particle size distribution can also be expected to have an effect. The main conclusion from these particular wall temperature profiles, however, is that a high draft setting can be expected to cause air inleakage, which affects the flame in two ways: first, by cooling the flame directly, and second by cooling the furnace walls and thus increasing the thermal (radiation) load on the flame. Consequently, controlling the draft in this type of experiment is critical.

Particle Temperature. Particle temperatures were measured for 7 of the 9 runs using the 2-color pyrometer, and TC measurements were also made in one series II run (12-19-92). The 2-color measurements ranged from approximately 50 to $200 \mathrm{C}$ higher than the corresponding wall temperatures in agreement with past records, with the exception of run 8-13-91 where most of the measured particle temperatures were nearly the same as the wall temperatures except in the first $20 \mathrm{~cm}$. The measured particle temperatures from four of the runs, 1-19-91, 8-19-91, and the two runs on 9-9-91, were mostly from 50 to $100 \mathrm{C}$ higher than the corresponding wall temperatures, while the measured particle temperatures for runs 7-31-91 and 9-4-91 were more than $100 \mathrm{C}$ higher than the corresponding wall temperatures. In these runs, the calculated flame temperatures are compared graphically with the measured values, with the calculations based as noted previously on a correlation of measured wall and flame temperatures (measured with a suction pyrometer) obtained in previous work with a nearly identical furnace. With an estimated 
accuracy of $\pm 50 \mathrm{C}$, however, these differences are not extreme and, although there are some clear differences or deviations, the generally overall adequate agreement supports the earlier correlations reported.

7.3 Particle Size Distribution. The variation of PSD through the flame was followed in 6 of the first 9 (Series I) runs, with the PSD determined with the Microtrac from batch samples collected using the sedimentation probe in the introduction cone and at successive vertical positions in the flame. Most of these samples show a fairly high degree of self similarity, with gradual increases or decreases in the average particle size. The results are shown in the relevant graphs in these first 6 runs on Rosin-Rammlex plots. One of the striking characteristics of many of. these plots is the apparent conversion of the nonlinearity of the raw coal plots into substantially linear plots of the flame samples. The other interesting characteristic is the relatively small variation, for the most part, in either the slope of the lines (the $R-R$ power index, $n$ ) or the Rosin-Rammler mean diameter.

Runs 1-17-91 and 1-19-91 using coal 13 in both cases show the most similar patterns of PSD variation with vertical distance in the flame although the excess air levels (at $16 \%$ and $-7 \%$ ) were substantially different (and with some difference in firing rate, at 14 and $16 \mathrm{lb} / \mathrm{hr}$ respectively). Both runs showed the following pattern: a slight increase of the average particle size from the feed to port 1 , then a gradual decrease from port 2 to either port 4 or 5 , and finally a gradual increase again from port 5 to 
ports 6 and 7 . The measurements were also repeated for run 1-1991 and although the PSD's are somewhat different, the general trend is the same. The common behavior in PSD between the two runs is remarkable in view of the marked difference in excess air, resulting in the temperature differences already noted.

Runs 7-31-91 and 8-6-91 which both used coal 1, at firing rates of 16 and $12 \mathrm{lb} / \mathrm{hr}$ and excess air levels of $8 \%$ and $7 \%$, are also fairly consistent with each other, but show a pattern different from that observed in the two runs with coal 13. Interpretation is more complicated in this instance, however, on account of a new sampling problem identified in these experiments when using the sedimentation probe. To stop reaction after sampling and to prevent further decomposition, the tray is part filled with water. Although the tray is, itself, water-cooled, nevertheless, the water in the slot probe will ultimatley boil and evaporate off. This limits the time that the probe can be kept in the flame to obtain the required sample. At low firing rates, however, the solids flux rate across the cross-section of the furnace is so low that an adequate sample cannot be obtained before the water boils off. This occurred with run 7-31-91, and especially with run 8-6-9l due to the lower fuel rate. Nevertheless, the basic pattern for run 7-31-91 (consistent with the port 0 and port 1 measurements for run $8-6-91$ ) is as follows: the average particle size increases from the feed to port 1 with furthur gradual increase to port 4 and then a decrease at port 6 . No samples were obtained from the lower ports for run 7-31-91 
because of the sampling problem already described at low particle flux rates.

In the last set of measurements, runs 8-13-91 and 8-19-91 both used coal 3 at a rate of $12 \mathrm{lb} / \mathrm{hr}$ and excess air rates close to 5\%. Samples could be obtained only at ports 0,1 , and 2 for run 8-13-91 (possibly due to the slightly higher temperature for this run) and showed the following pattern: the average particle size increased from port 0 to port 1 and then decreased again at port 2. The PSD measurements for run 8-19-91 showed the following pattern which is consistent with the pattern for run 731-91: the average particle size increased from port 0 to port 1 and then from port 1 to port 4 is a gradual increase; at port 5 or 6 was a dramatic decrease in average particle size followed by another gradual increase from port 7 to port 11 .

A basic pattern of PSD variation in the flame emerges from these measurements: there always appears to be an increase, followed by a decrease (either gradual as in runs 1-17-91 and 119-91, or rapid as in runs 7-31-91 and 8-19-91) and finally a gradual increase in the average particle size. The reason for these PSD variations and for the differences between the various runs in where and when these changes occur is uncertain and requires furthur investigation. However, the relatively small changes in mean particle size imply mainly internal burning of the coal, as is to be expected from these types of coal. Furthurmore, the general trends shown by the measured PSD results are in substantial agreement with theoretical predictions of the PSD as illustrated in fig. 4.4 of $\sec 4$. 
7.4 SO2 concentration. The $\mathrm{SO} 2$ concentrations were of particular importance in these studies though the fully detailed investigation planned was aborted on account of the flame structure problems reported. Nevertheless, vertical so2 concentration profiles were measured for 7 of the 9 runs in the Series I experiments. The values obtained, however, were typically much lower than expected. Runs 7-31-91 and 8-6-91 both used coal 1 ( $1.67 \%$ sulfur) and both were at approximately $7 \%$ excess air so that the calculated peak so2 concentration (assuming complete release) for each run was about $1350 \mathrm{ppm}$ (indicated in each graph by the rightmost symbol); but the measured peak SO2 concentrations were 800 and $870 \mathrm{ppm}$, which are approximately $38 \%$ lower than the expected peak value. Runs 8-13-91 and 8-19-91 both used coal 3 (1.74\% Sulfur) and both were at approximately $5 \%$ excess air so that the calculated peak so2 concentration for each run was about $1450 \mathrm{ppm}$. However the measured peak SO2 concentrations were 1020 and $1200 \mathrm{ppm}$, and these are approximately $24 \%$ lower than the expected peak value. Run 9-4-91 used coal 4 (1.81\% sulfur) and the calculated peak so2 concentration was about $1600 \mathrm{ppm}$. The measured peak so2 concentration was $1060 \mathrm{ppm}$ which is approximately $34 \%$ lower than the expected value. Since the amount of draft was, comparatively, the lowest for runs 8-13-91 and 8-19-91, and since the measured peak SO2 concentrations were $24 \%$ lower than expected for these runs, compared to $38 \%$ and $34 \%$ lower for runs 7-31-91, 8-6-91, and 9-4-91, the differences in expected and measured concentrations is probably partly due to air inleakage. 
The overall imbalance, however, was the trigger that led to the investigation of flame structure. The first thought was that the missing so2 might be in the wall flows and, indeed, further examination of the horizontal profiles established that the concentrations on the axis were low. The problem of the horizontal profiles is discussed more fully in sec. 7.6.

This thinking was re-inforced by the first run on 9-9-91 using coal 9 ( $3.78 \%$ sulfur) with a calculated peak so2 concentration of about $4360 \mathrm{ppm}$ (-.68 excess air); the two runs on this date were designed to compare 502 levels without and with limestone sorbent in the coal mixture. The measured 502 profile for the first run on 9-9-91 shows a counter-intuitive pattern which is in fact similar and in qualitative agreement with the reaction efficiency profile for this run. These measurements provided the first definite support for a possible alternate flame structure. For the first three gas concentration measurements below the water cooled tube bank, the probe was inserted through ports in a removable brick intended for better visual access and more frequent measurements in this uppermost region of the flame. However the outside face of the brick extended about an inch or two beyond the outer wall of the furnace and, consequently, the tip of the probe for these upper three measurements did not extend to the furnace centerline but was an inch to two closer to the inside furnace wall and thus provided the first indicated variations in the gas concentrations in the horizontal planes. The peak (near wall) so2 concentration measured for the first run on 9-9-91 was about $2500 \mathrm{ppm}$ at only 
$6.4 \mathrm{~cm}$ below the tube bank and was $1000 \mathrm{ppm}$ higher than the peak centerline so2 concentration, at 1950 ppm, which was approximately 55 lower than the calculated peak sor concentration of $4360 \mathrm{ppm}$.

These data were to provide initial values for comparison with the second run on 9-9-91, also using coal 9 (3.78\% sulfur) although for operational reasons it was not possible to keep the same coal rate and combustion stoichiometry (at $18.1 \mathrm{lb} / \mathrm{hr}$ and substantially fuel rich at $-28.3 \%$ excess air). Under these firing conditions, the calculated peak so2 concentration was about $6090 \mathrm{ppm}$ for complete release without the sorbent. With the 1 imestone included, the measured peak so2 concentration of $1800 \mathrm{ppm}$ is approximately 70 lower than the expected peak without limestone injection. Because of the non-uniform horizontal concentrations, a definitive interpretation cannot be provided at this time; nevertheless, the indications are that there is probably significant reduction of 502 which merits furthur investigation.

7.5 Gas Analysis Reaction Efficiency and Local Excess Air. An important measure of the progress of the reaction, and often the best single measure is the reaction efficiency. The reaction efficiency can be determined either from the gas analysis or from the solid samples (proximate) analysis, and comparison of the two provides an important cross check both on the accuracy of the calculation and on the accuracy of the initial measurements. In this sub-section, the reaction efficiencies obtained from the combustion gas analysis are reported, and the values from the 
proximate analysis are reported in sec. 7.6. The reaction efficiency can also be monitored by means of determination of the local excess air.

Profiles for both reaction efficiency and excess air were determined for 8 of the 9 series I runs. Reaction efficiency and local excess air results are shown as a fraction of unity with a reaction efficiency fraction of 1 (100\% reaction efficiency) for complete combustion. The reaction efficiency profiles, which are determined from the measured 02 and $\mathrm{CO} 2$ gas analyses, are typically only slightly lower than expected. The exception is run 8-6-91 for which the 02, $\mathrm{CO2}$, and so2 concentrations were measured near the end of the run when the coal supply was rapidly dwindling and the flame was becoming intermittent. (The lower SO2 profile was the second r sasured so2 profile for run 8-6-91; lying above this is a more reasonable profile measured earlier in the run.) No furthur interpretation is required due to the sporadic appearance of the flame during the second set of measurements.

Runs 7-31-91, 8-13-91, and 8-19-91 have relatively steep reaction efficiency profiles and peak values which are nearly the same as the calculated peak reaction efficiencies. Run 7-31-91 has a peak measured reaction efficiency of 0.96 which is within unity allowing for reasonable error of measurement. Run 8-13-91 has a peak measured efficiency of 1.00 which is the same as the expected value, and run 8-19-91 has a measured efficiency of 0.93, which is only marginally lower than the expected complete 
combustion; these small departures are most likely due to small amounts of air inleakage.

In contrast, runs 1-19-91, 9-4-91, and the two runs on 99-91 have much less steep reaction efficiency profiles in comparison to their corresponding wall temperature profiles. The first three of these four runs shows some evidence of the alternate flame structure more fully discussed later. Run 1-1991 has a peak measured reaction efficiency of 0.78 which is 16 percentage points below the expected value of 0.93 ; and run 9-491 has a peak mer sured efficinncy of 0.77 which is 22 percentage p.xints below the expected value of 0.99. The first run of 9-9-91 has a peak (centerline) measured efficiency of 0.82 which is $18 \%$ percentage points below the expected value of 0.99 , and the second run has a peak measured efficiency of 0.60 which is $16 \%$ percentage points below the expected value of 0.72 . These lower reaction efficiencies are likely due in part to some air inleakage, but they may also be due to the alternate flame structure. For run 9-9-9l this is clearly evident by the first three measurements which were off the furnace centerline for reasons discussed above as these were taken through ports in the removable brick. Run 9-4-91 shows no evidence in the gas concentration measurements (the gas sampling probe may have been inserted furthur so that the tip was at the furnace centerline) but the reaction efficiency profile from the solid sample proximate analysis (also discussed in sec. 7.6) shows much higher reaction efficiencies for the first three ports and is indicative of the alternate flame structure. The first measurement for run 
1-19-91 also indicates the possibility of the alternate flame structure for this run.

It is important to note the relatively smaller differences between the measured and expected reaction efficiencies (ranging from 0 to 22 percentage points difference for the various runs) compared to the differences between the measured and expected so2 concentrations (ranging from 24 to 55 percentage points difference). If these ranges of error between measured and calculated values were comparable then one could reasonably conclude that the differences were simply due to air inleakage and the alternate flame structure; however, these particularly low so2 measurements indicate that there may not be complete sulfur release during the residence time in the furnace used in these experiments, and this is possible if only the organic sulfur is released in the combustion. clearly, this provides an excellent basis for furthur investigation of these differences and characteristics.

7.6 Proximate Analysis Reaction Efficiency. As noted above, the reaction efficiency can also be calculated from the proximate analysis and, in this sub-section, values obtained are reported.

Solid samples were collected for proximate analyses for 5 of the 9 series I runs. Three different probes were used: a short suction probe (23.5 inches long), the sedimentation probe, and a long suction probe (50 inches long). The proximate analyses of the samples collected using the shorter suction probe for runs 8-13-91 and 8-19-91 show little variation with vertical position in the flame, indicating that the probe was too short 
for rapid quenching of the solid sample. The VM content of the tray samples collected for run 8-13-91 compared with the VM content of the corresponding suction probe samples furthur illustrates the inadequacy of this shorter suction probe although the difference could also be partly due to the alternate flame structure in which the extent of reaction is much higher near the furnace walls compared to that at the centerline of the furnace. If the flame for run 8-13-91 had the alternative structure, and if the suction probe samples were collected closer to the furnace wall, then these samples would probably have less VM content compared to the sedimentation probe samples which collect more of an average sample across the width of the furnace. This point became evident only on analysis of the results obtained and time did not permit a follow up on these lines.

The proximate analyses of the solid samples collected using the longer suction probe for runs $9-4-91$ and the two runs on 9-9-91 mostly show the expected variation and trends. The length for this longer probe was chosen based on the calculated length required to obtain high sample cooling rates and rapid quenching of the solid sample. The proximate analysis reaction efficlency for run $9-4-91$ is in basic agreement with the reaction efficiency profile calculated from the corresponding gas analysis. However the proximate analysis reaction efficiency is slightly higher; this indicates that the gas analysis reaction efficiencies may be in error due to air inleakage. Also, the first three points in the proximate analysis reaction efficiency are inconsistentiy higher than the rest of the points which 
indicates the likelihood of the existence of the alternate flame structure (although the gas analysis reaction efficiencies do not show this pattern). The proximate analysis reaction efficiency for the first run on 9-9-91 was also in basic agreement with the corresponding gas analysis reaction efficiency profile. Again, the proximate analysis reaction efficiencies are higher, indicating slightly erroneous gas analysis reaction efficiencies due to air inleakage. Both reaction efficiencies show evidence of the alternate flame structure. Finally, the basic trend of the proximate analysis reaction efficiency for the second run on 9-9-91 is in agreement with the corresponding gas analysis reaction efficiency, although the magnitudes are questionable. Determination of the reaction efficiency in flames with limestone injection will require further investigation.

7.7 Horizontal profiles. These profiles are included in Figs. 7.23 to 7.44 , and were obtained in the series II experiments designed to identify to the extent possible the flame structure variations caused by different firing procedures and rates. At this time it is still not possible to say for certain the precise source of the different flame profiles, although one factor does seem to be the stoichiometry used initially on light-off. From the results obtained and in agreement with prior work it appears that the flame temperature peaks at rich conditions (about $-30 \%$ excess air), and this may be the route to stabilization of a different flame structure.

In the experiments described following, the principal focus was on the effect of changing firing conditions using only 
two coals, numbers 11 (Figs. 7.23 to 7.38 ) and 12 (Figs. 7.39 to 7.44). The majority of the results are for the horizontal profiles, but the results do include relevant vertical profiles, some of which (notably the temperatures) have already been discussed above.

7.7.1 Verification of Alternate Flame structure. Evidence for the alternative (cone-shaped) flame structure is seen in the first of the series II runs of 12-6-91 and 12-12-92, both using coal 11, and shown by the non-uniform sox profile in the first run (though the $\mathrm{CO} 2$ and oxygen are not too variable in this graph) and the very marked variations of all gas compositions in the second run.

These particular experiments were also structured to determine the repeatability of given firing conditions and particularly of the proximate analysis measurements, as well as determination of the detailed measurements on the horizontal planes. The full suite of results is shown in Figs. 7.23 to 7.28. In particular, Fig. 7.24 for the reaction efficiency from solid sample analysis (burn-off) for run 12-6-91 is in excellent agreement with the same determination for run 12-12-92, shown in Fig. 7.27. A repeat of the reaction efficiency determination from the solid samples for run 12-12-91 is shown in Fig. 7.28, and is also seen to be in excellent agreement with the previous profiles. In addition to establishing the repeatability of the solid sampling procedure and proximate analysis determinations, this also supported the repeatability of such measurements in the horizontal plane. 
Since draft had already been identified as a major factor in controlling the vertical profiles, this was further examined as already partly discussed earlier. The relevant plots are shown in Fig. 7.29 (run of 12-17-91) and the lower two graphs show the influence on gas concentration, notably at the wall, which is presumably due to air inleakage from the increased draft (comparing the bottom LH graph with the bottom RH graph). These two plots, however, show that the effect of the draft is evidently focussed at the walls, with the concentrations in the center flow largely unaffected. This implies that inleakage is most probably through the sampling ports with little penetrating to the center flow.

Although the preceding results indicated the non-uniform profiles indicative of non-planar flame fronts, this alternative flame structure is more firmly established by the results shown in Figs. 7.30 and 7.31 , in the run on 12-19-91, though with one proviso, where the horizontal gas concentration profiles show the marked variation from wall to center line. The (relative) temperature profile at port 1, obtained with a TC however, shows surprising uniformity, nonetheless, so there is a further question here yet to be resolved. The firing conditions were changed during this run, with an increase in both air rate and fuel rate, but with excess air, overall, being reduced somewhat (from $-27 \%$ to $-29 \%$ ). These gas and solid sample data are also used to compare the local reaction efficiency calculated from the two different data sources, and these results are shown in Fig. 7.32. The two graphs are shown independently in the top two 
graphs, and they are compared in the lower graph. The numerical agreement is adequate and typical of such comparisons but, more important, the trends are in full agreement. Moreover, on account of the air inleakage, we should expect the gas-calculated values to be low since they are calculated on the assumption of invariant air supply. The difference between the two lines may thus be a means of determining the magnitude of the inleakage.

7.7.2 Effect of Firing conditions. To explore further the possible effect of firing conditions (firing rate and stoichiometry) on the horizontal flame profiles, runs 12-21-91 and 12-31-91 (Figs. 7.33 to 7.36) were carried out at a range of different firing rates and excess air values. In particular, the excess air levels ranged frcm about $-2 \%$ to $-49 \%$. In general, the temperatures increased with diminishing excess air though this was also influenced by the firing rate, as can be seen comparing Figs. 7.34 with 7.35 . The horizontal profiles then show somewhat variable patterns, mostly non-linear to highly non-linear, though there are some exceptions such as in Fig. 7.35 for port 9 . Nevertheless, the values at the higher firing rates do seem to show greater variation across the horizontal than at the lower firing rates. At the same time, the flatter profiles appear to occur for the more fuel rich conditions.

7.7.3 Effect of Initial conditions. These test were followed by an investigation of the possible influence of initial conditions on light-up, and the results are shown in Fig. 7.37 (run 1-4-92). In this test, the furnace was preheated with a high intensity methane gas burner to a substantially higher 
initial wall temperature than used before in light-off, of 1300 to $1400 \mathrm{C}$. The temperature profile still reflects the coal firing conditions, however, and is clearly independent of the initial wall temperature. This, however, then suggested that the combination of firing pattern and (higher) fuel rate could be a factor and this was examined later (run of 2-14-92) in which the possible effect of local buoyancy was also examined.

7.7 .4 Effect of coal Type. This test was included in a sequence of experiments from 1-8-92 through 2-14-92. The principal parameter examined was coal type using, for this purpose, coals 11 and 12 . The results are in figs. 7.38 to 7.43 . Coal 12 was chosen for its evident high "reactivity", notably shown by its continued ability to flash back into the cone even after the design and other changes described earlier. The firing conditions are given on the relevant graphs and included change from about $101 \mathrm{~b} / \mathrm{hr}$ coal rate to $16 \mathrm{lb} / \mathrm{hr}$, with excess air variations from $-10 \%$ to $-20 \%$, and at $-52 \%$ in the final test of 214-92. No clear pattern of influence on the results was observed, however, as will be evident from inspection of figs. 7.38 through 7.43 , except for further evidence that the horizontal gas concentration profiles tend to become more uniform further down in the flame. This can represent time for improved cross-mixing. However, there was lower repeatability for the coal 12, and this is believed to be due to its greater selfagglomeration properties, either from higher inherent wetness or from some other property not yet identified. In consequence, the ability to flash back into the cone is seen more as a consequence 
of this agglomerating behavior that first partly plugs the tube bank and permits passage of flame at locations where the air speed has been reduced on that account. This also affected the repeatability since the coal could be seen to accumulate on the top of the sampling probe and then be sucked into the probe to produce anomalous sampling results.

7.7 .5 Effect of Buoyancy. The final test before experiments were terminated for time was that of 2-14-92 and this was carried out in a re-build of the furnace designed to reduce the distance from the tube bank to the furnace exit from about $1.9 \mathrm{~m}$ to about $1.3 \mathrm{~m}$, this being the height of the furnace used in the original experiments 30 years ago. The argument here was that the higher furnace could be responsible for buoyancy effects at the top of the furnace that were producing backmix that generated flame holding at the wall. In this run, the draft was set fairly high but there was a considerable change in the appearance of the flame. In particular, the flame was very much brighter in the volatiles region, and the flow paths shown by the particle streaks were effectively parallel, unlike the indications of buoyancy vorticity seen in the earlier experiments. More significantly, the shape of the horizontal profiles, as seen in Fig. 7.44, are reversed from the previous ones, with the $\mathrm{CO} 2$ declining instead of rising towards the wall and the oxygen rising instead of declining. This can be explained by assuming high wall inleakage due to the high draft but, nevertheless, the inference is that without the high draft, the profiles would have been more uniform across the furnace and, 
thus, closer to the pattern of the earlier flames. At this time, this behavior is considered indicative not definitive, but it does suggest that the combination of furnace height, firing rate, stoichiometry, and possibly fuel type is largely or totally responsible for the unexpected cone-shaped flame structures described in this work. 


\section{Run: $\quad 01-17-91 \quad$ Coal $13 \quad$ EXo $=15.7 \%$ Firing Conditions: $\quad 13.9 \mathrm{lb} / \mathrm{hr} \quad 34.8$ scfm}

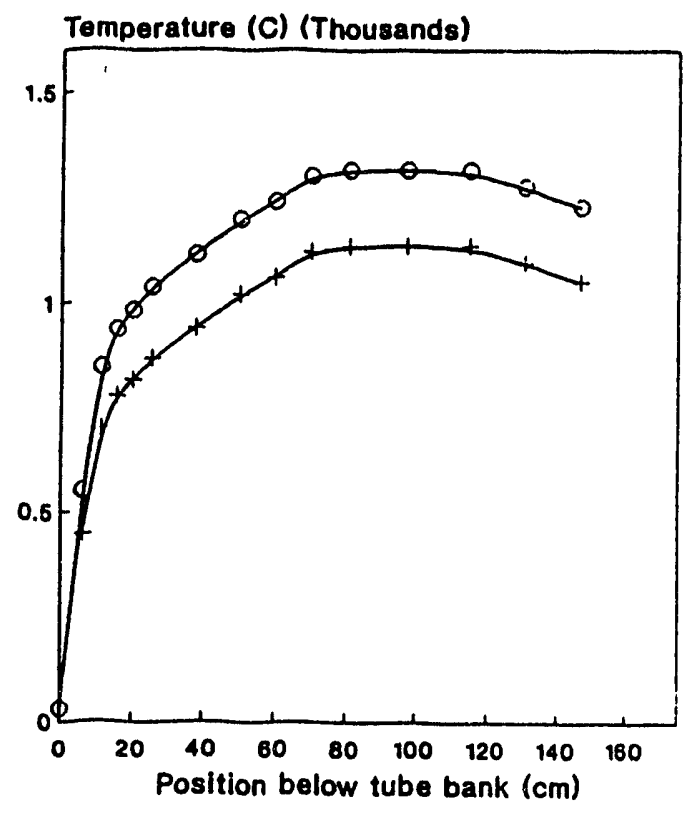

- Wall T Flame $T$ o Calculated

FIG. 7.1 
PSD: Rosin-Rammler Plots

Run: $\quad$ 01-17-91

Firing Conditions:

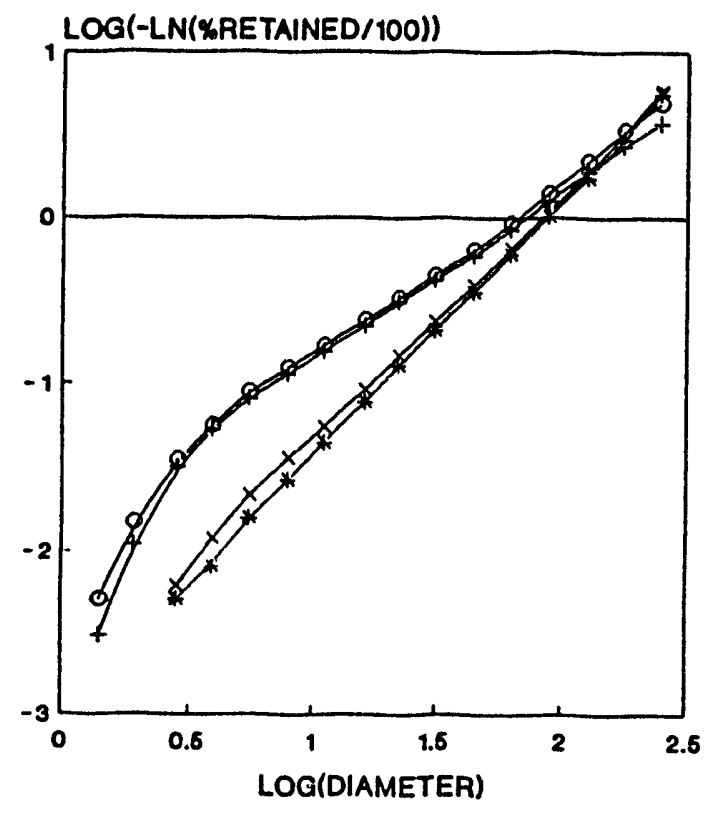

0, Feed $x_{0}$ - Port 1

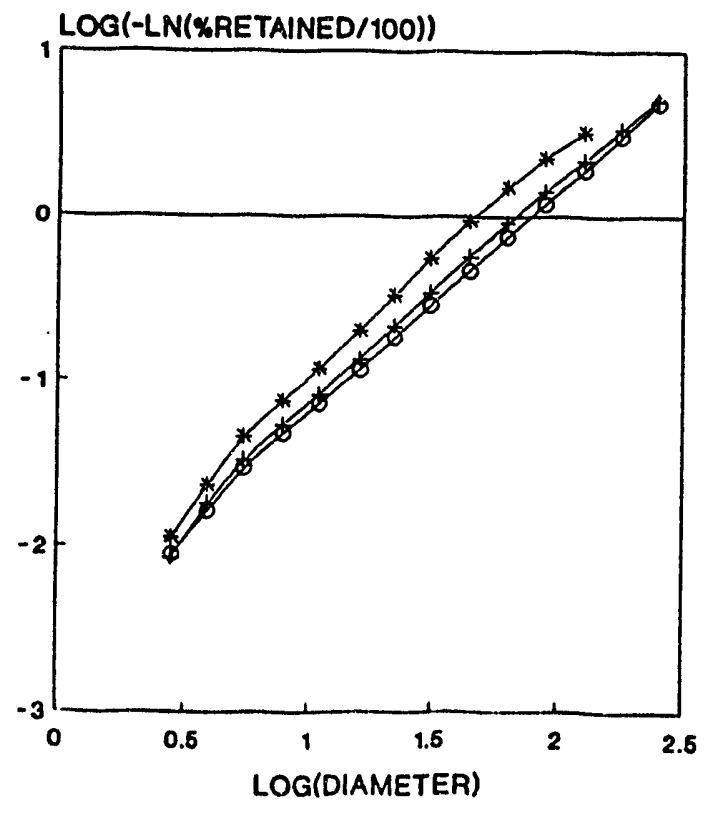

- Port 3 - Port 4 - Port 5
Coal 13 EXo $=15.7 \%$

$13.9 \mathrm{lb} / \mathrm{hr} \quad 34.8 \mathrm{scfm}$

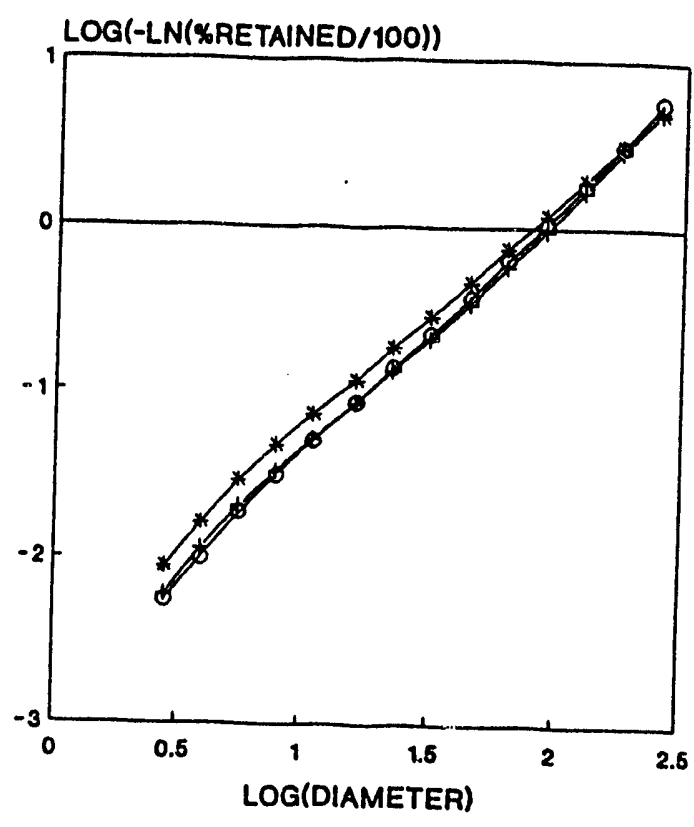

- Port 1 - Port 2 - Port 3

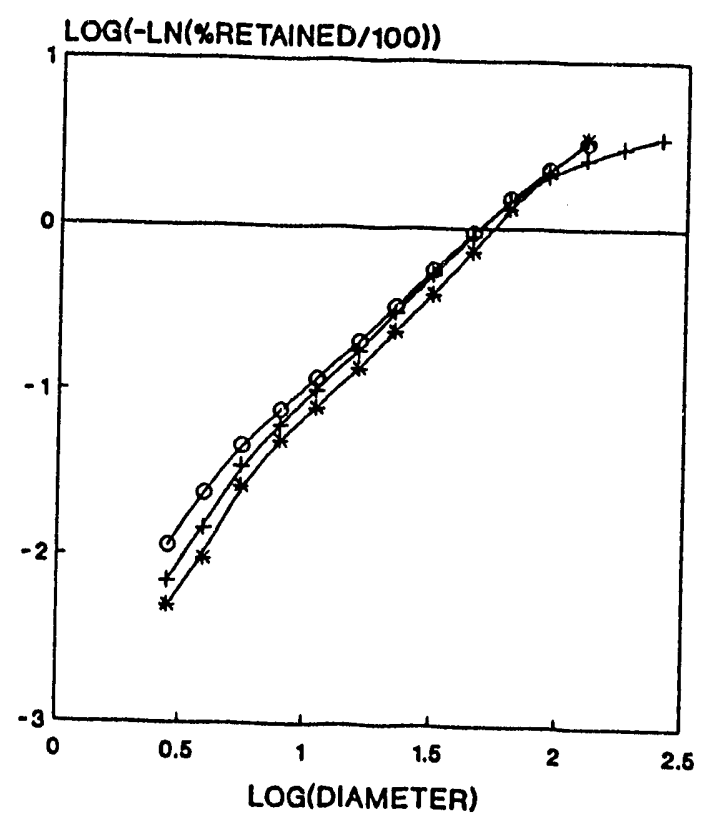

- Port 5 - Port 6 - Port 7

FIG. 7.2 


\section{Run: $\quad 01-19-91 \quad$ Coal $13 \quad E X o=-6.8 \%$ Firing Conditions: $\quad 16.3 \mathrm{lb} / \mathrm{hr} \quad 32.8 \mathrm{scfm}$}

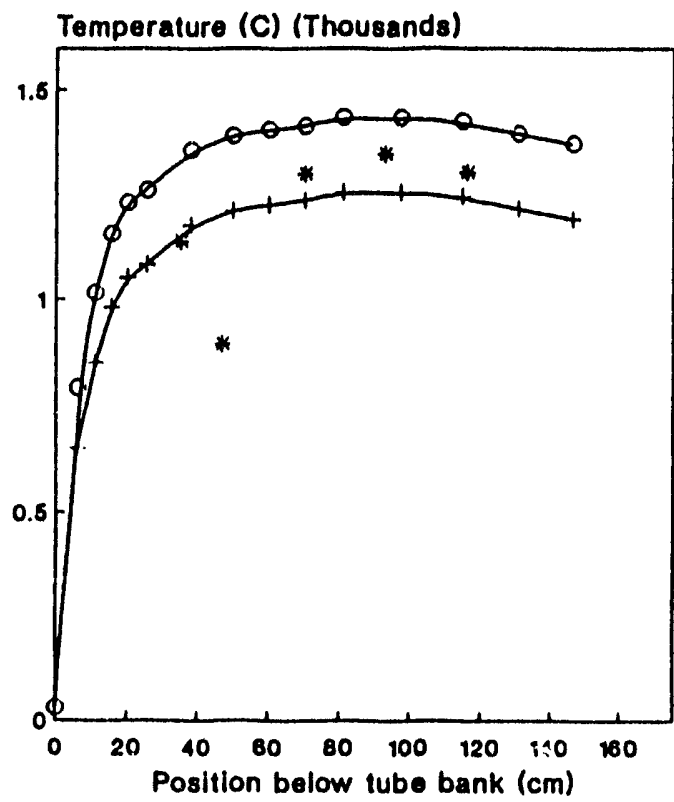

- Wall T Flame $T$ o Calculated
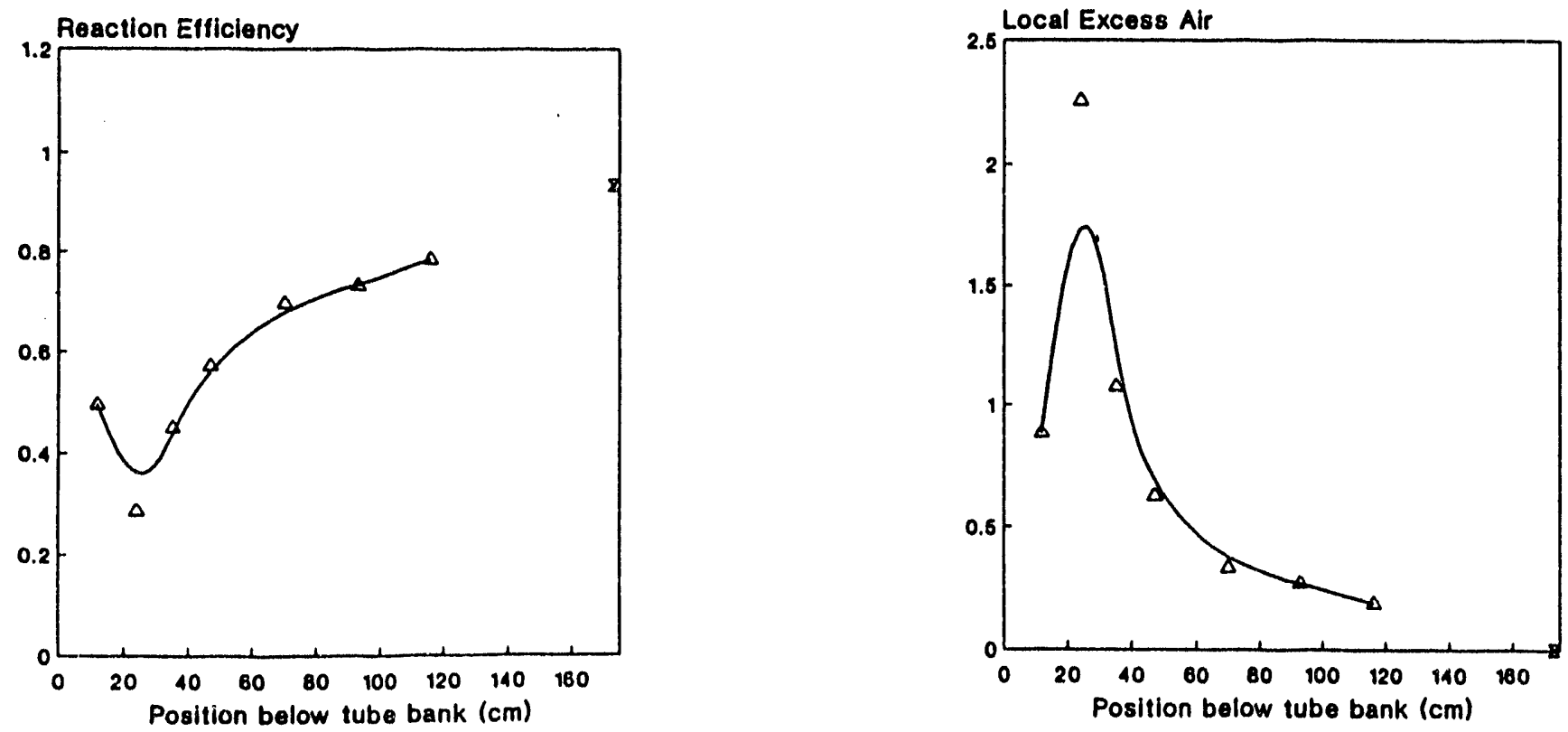

FIG. 7.3 


\section{PSD: Rosin-Rammler Plots}

Run: $\quad$ 01-19-91 Firing Conditions:
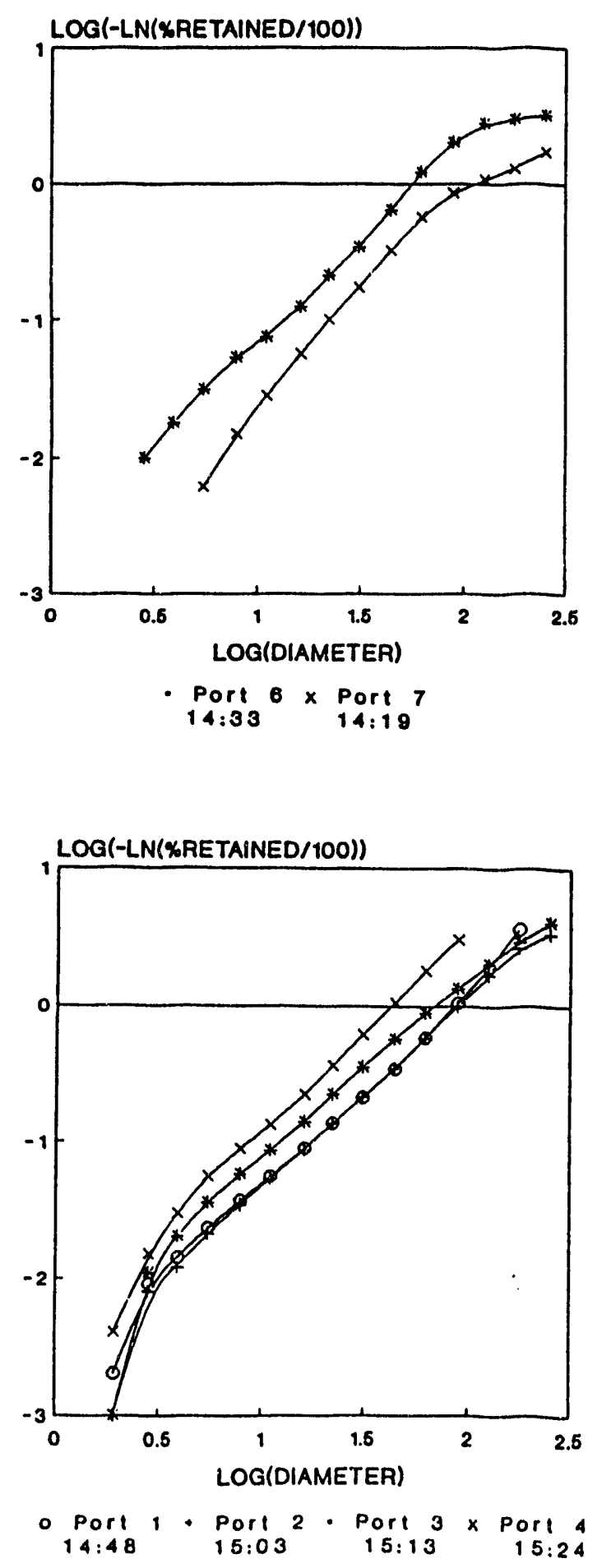

Coal 13 EXo $=-6.8 \%$ $16.3 \mathrm{lb} / \mathrm{hr} \quad 32.8 \mathrm{scfm}$
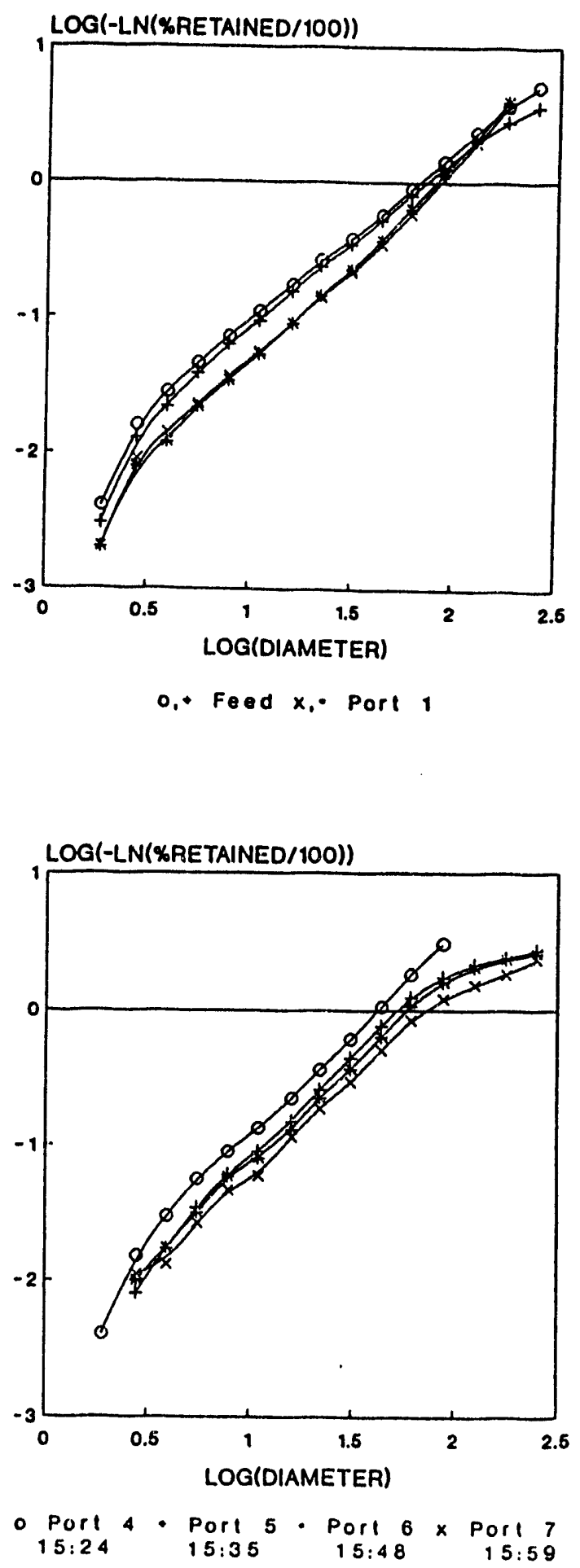

FIG. 7.4 
PSD: Rosin-Rammler Plots

Run: $\quad$ 01-19-91 Firing Conditions:
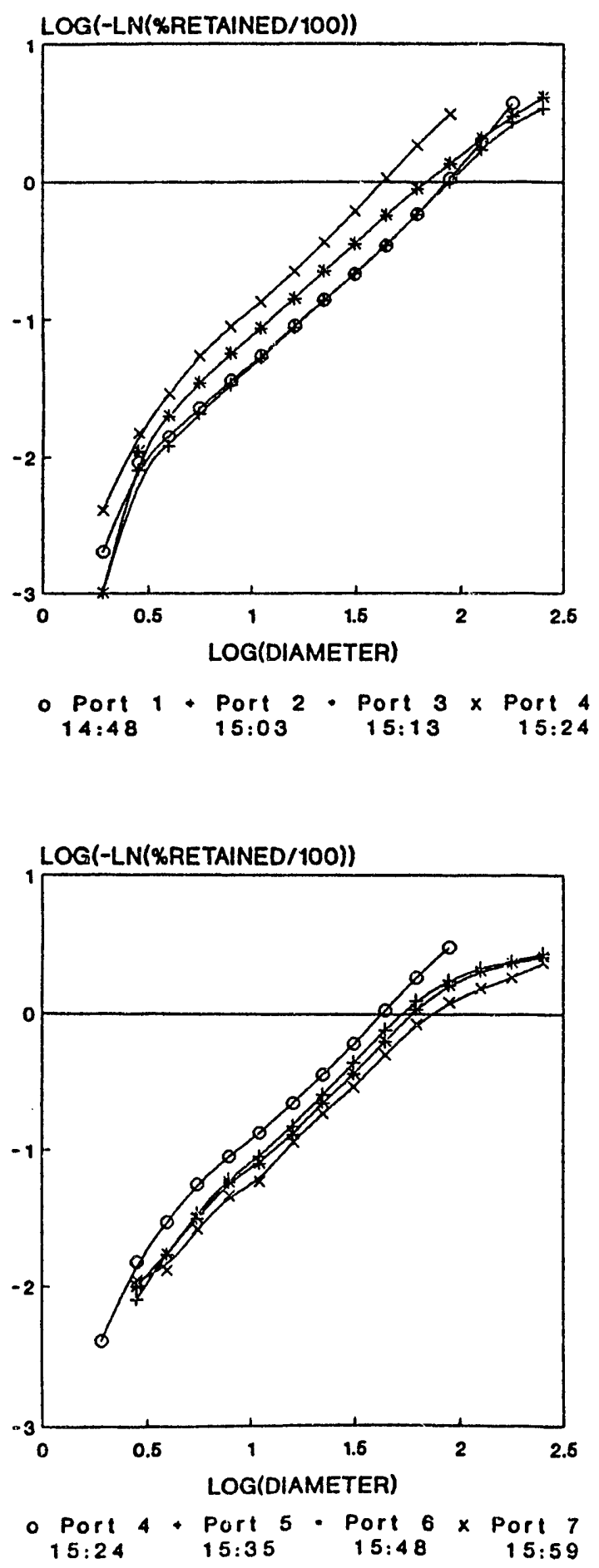

Coal $13 \quad E X o=-6.8 \%$ $16.3 \mathrm{lb} / \mathrm{hr} \quad 32.8 \mathrm{scfm}$
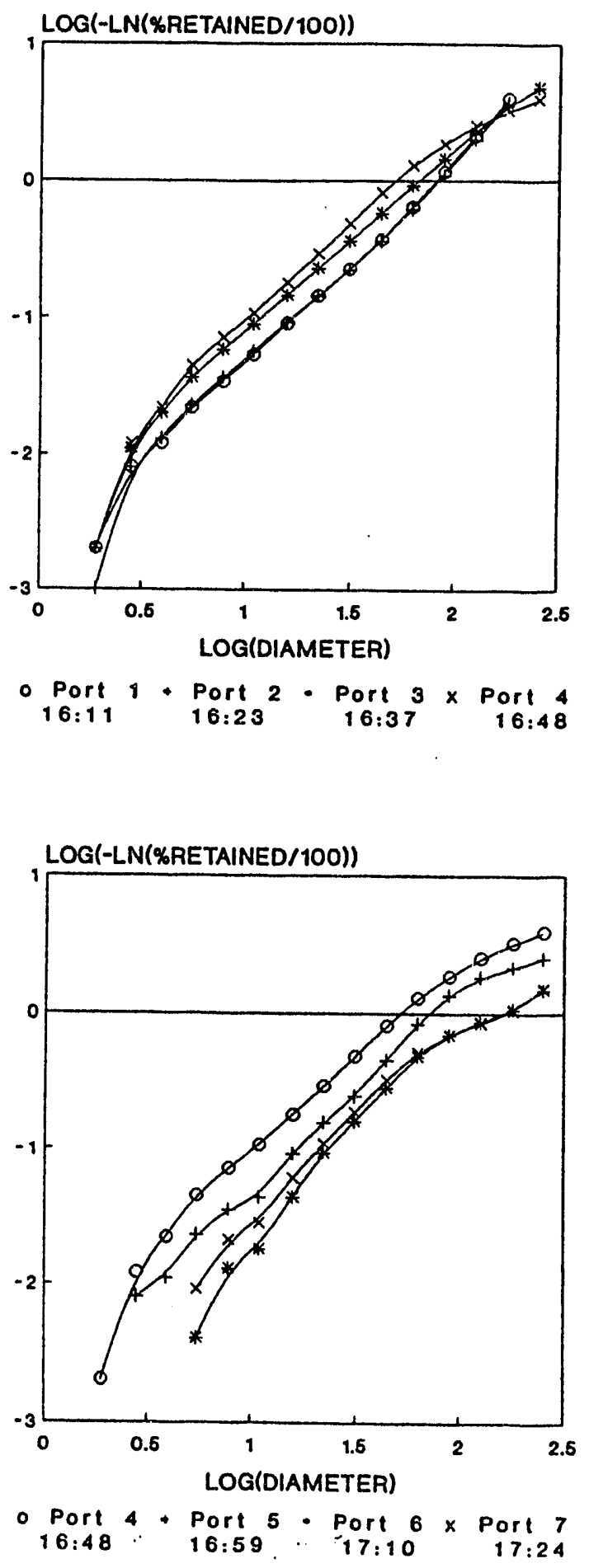

FIG. 7.5 


\section{Run: $\quad 7-31-91 \quad$ Coal $1 \quad E X o=7.8 \%$ Firing Conditions: $\quad 16.4 \mathrm{lb} / \mathrm{hr} \quad 43.0 \mathrm{scfm}$}
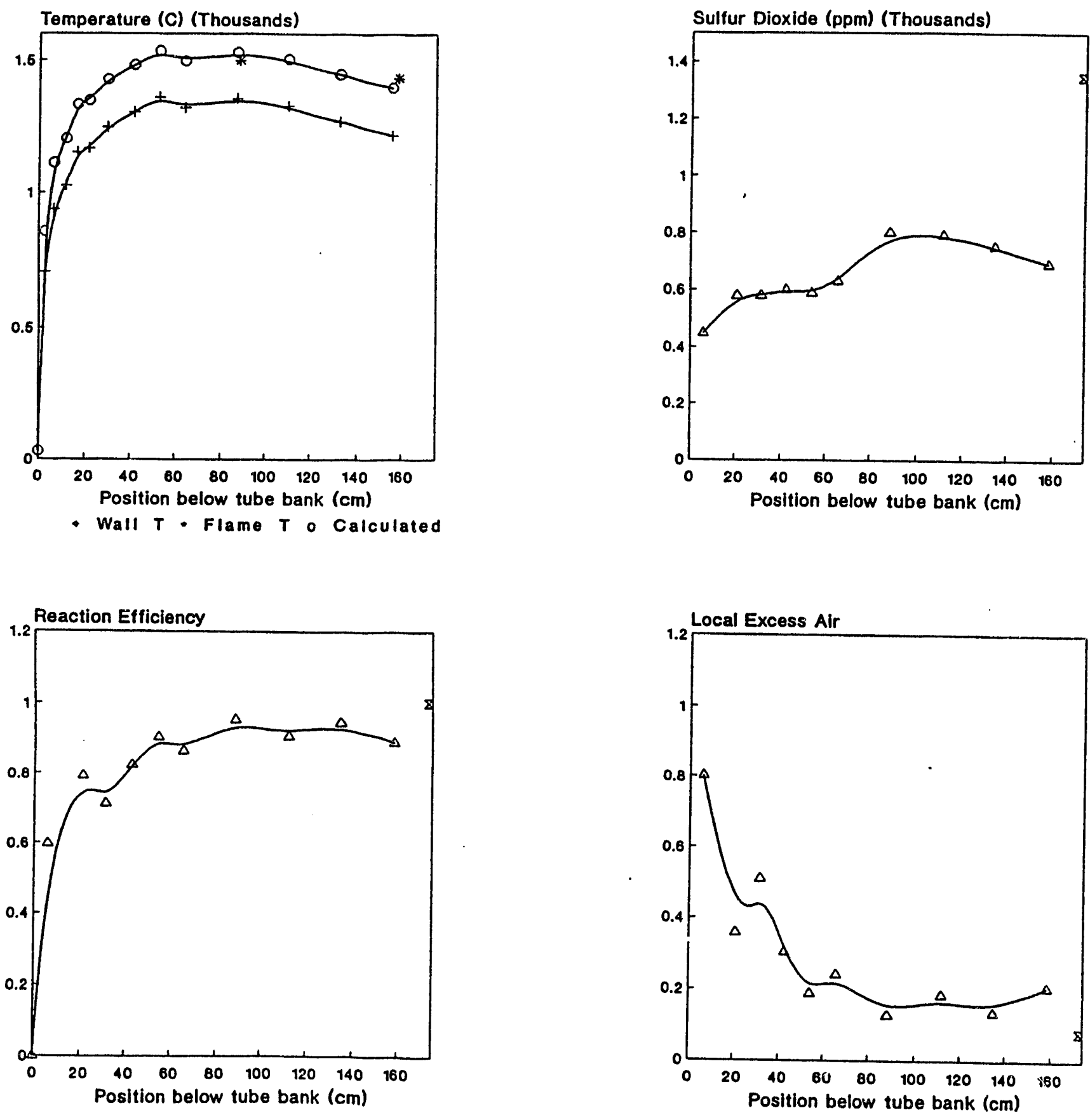

Draft: -.06 to -.31 inches water column

FIG. 7.6 


\section{PSD: Rosin-Rammler Plots}

$\begin{array}{lcll}\text { Run: } & 7-31-91 & \text { Coal } 1 & \text { EXo }=7.8 \% \\ \text { Firing Conditions: } & 16.4 \mathrm{lb} / \mathrm{hr} & 43.0 \mathrm{scfm}\end{array}$
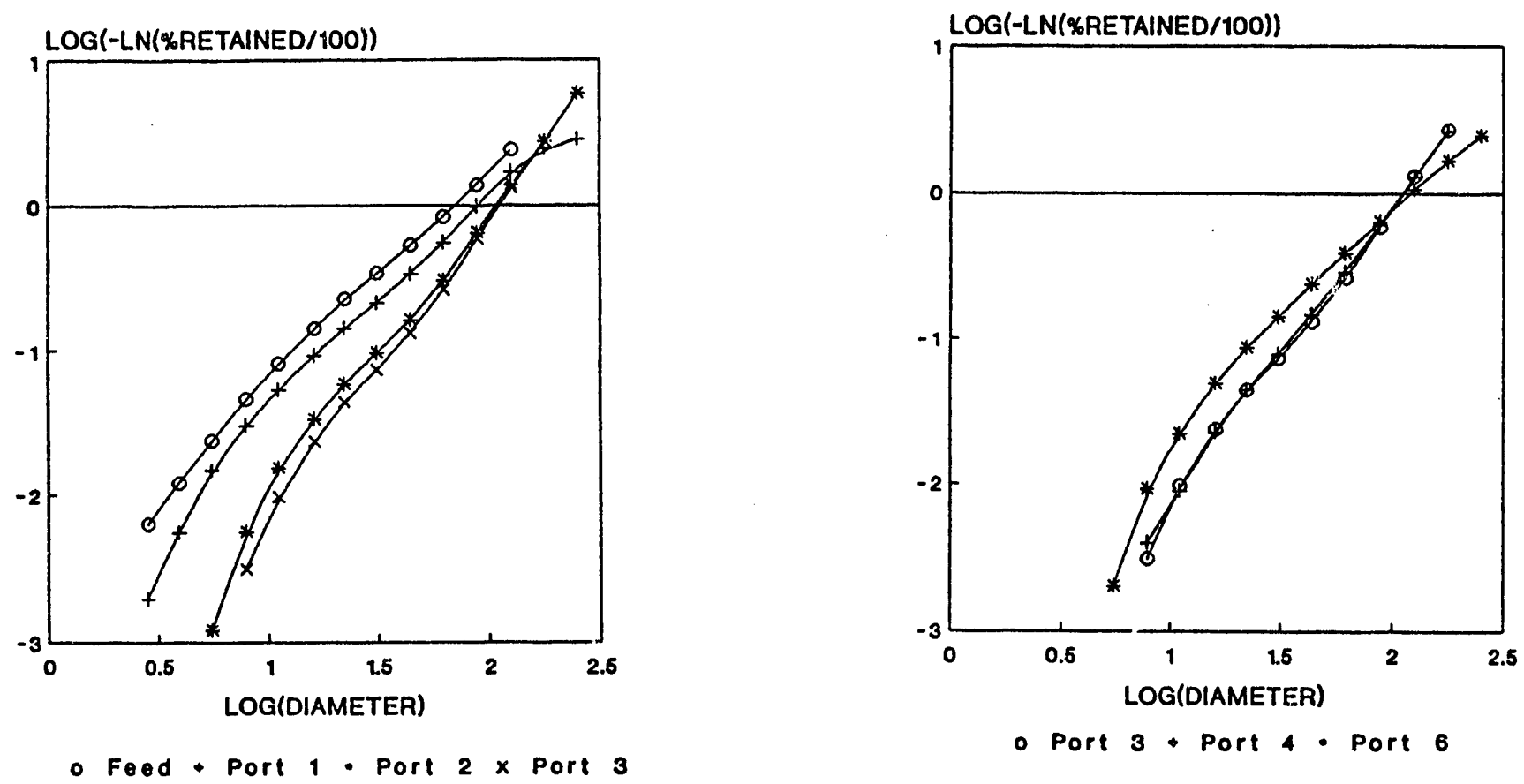

- Port 3 - Port 4 - Port 6

FIG. 7.7 
Run: $\quad 8-6-91$

Firing Conditions:
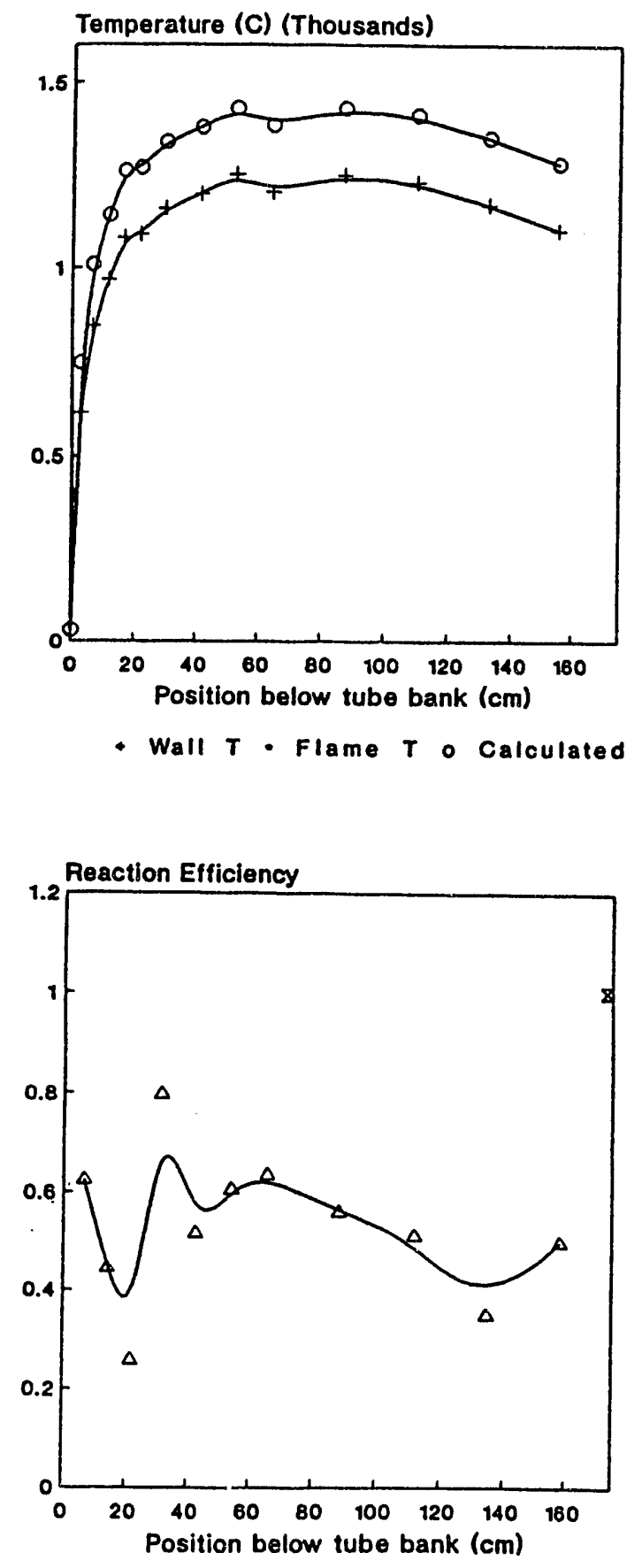

Coal $1 \quad E X o=6.9 \%$ $12.0 \mathrm{lb} / \mathrm{hr} \quad 31.0 \mathrm{scfm}$
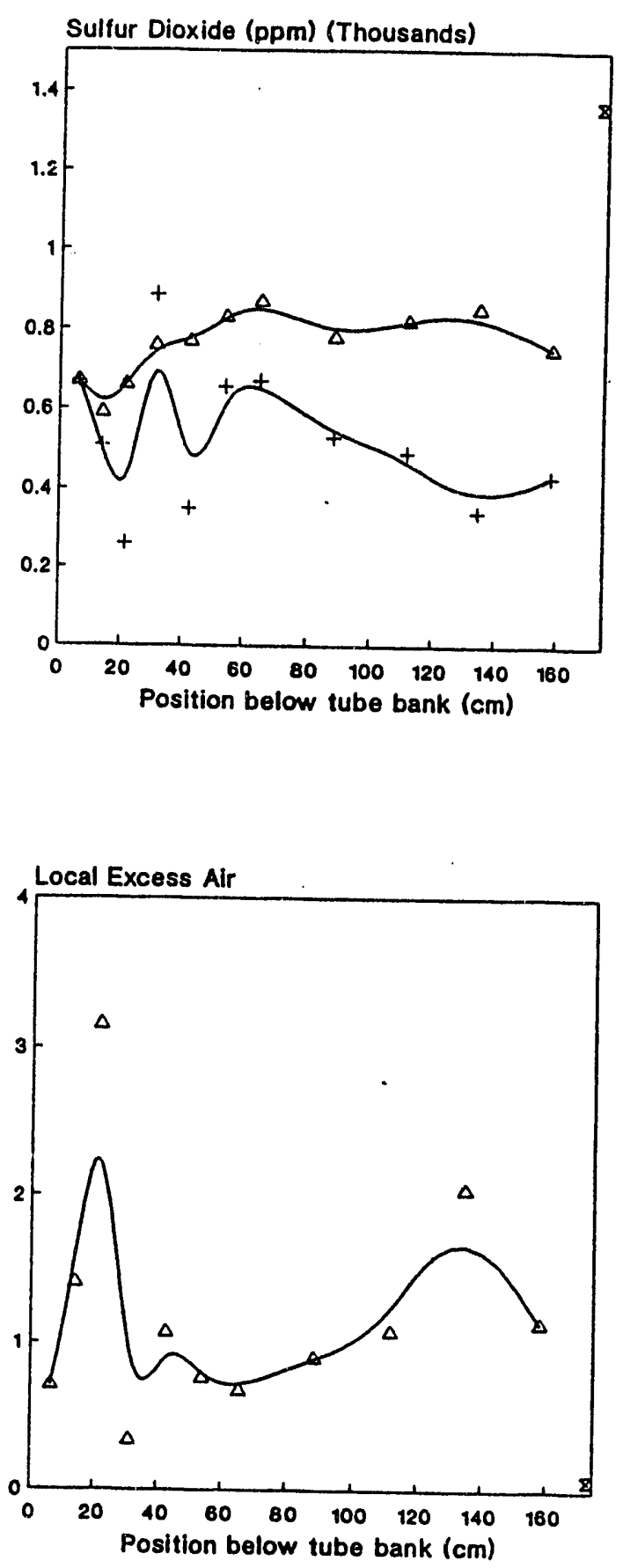

Draft: -.13 to -.33 inches water column

FIG. 7.8 


\section{PSD: Rosin-Rammler Plots}

$\begin{array}{lcll}\text { Run: } & 8-6-91 & \text { Coal } 1 & \text { EXo }=6.9 \% \\ \text { Firing Conditions: } & 12.0 \mathrm{lb} / \mathrm{hr} & 31.0 \text { scfmi }\end{array}$

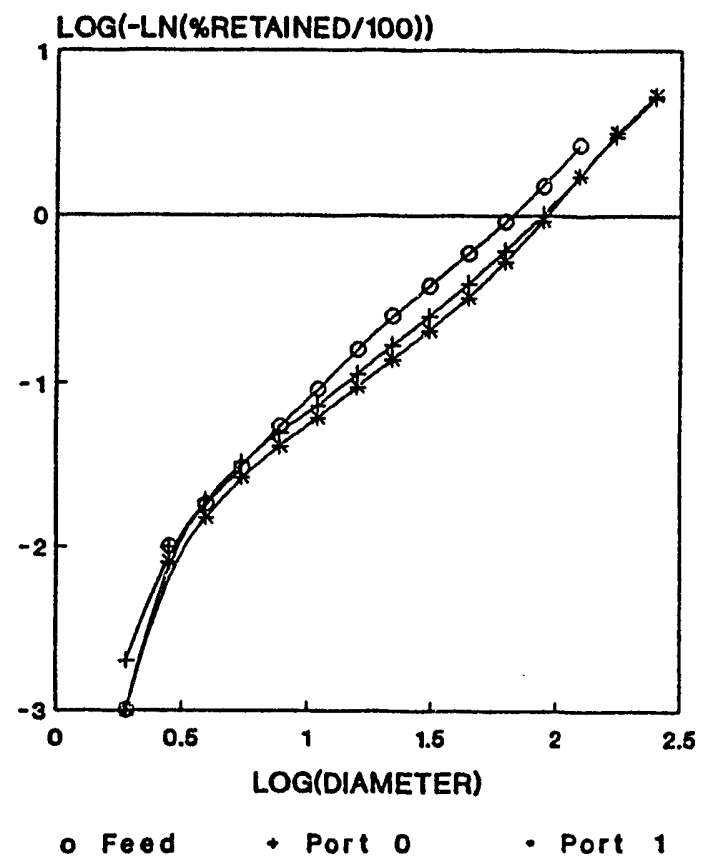

FIG. 7.9 


\section{Run: $\quad 8-13-91 \quad$ Coal $3 \quad E X o=5.7 \%$ Firing Conditions: $\quad 12.0 \mathrm{lb} / \mathrm{hr} \quad 30.8 \mathrm{scfm}$}
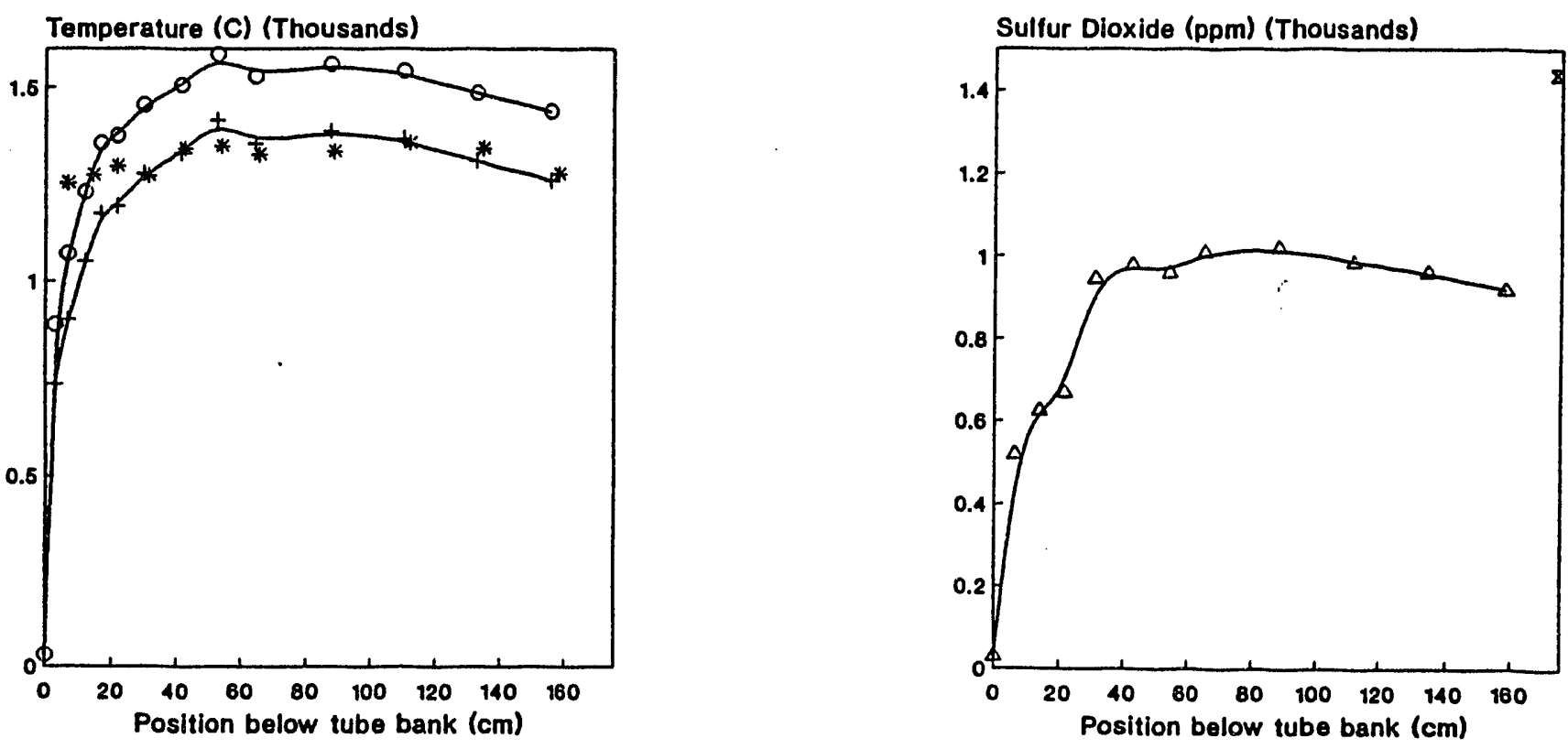

- Wall T Flame T o Calculated

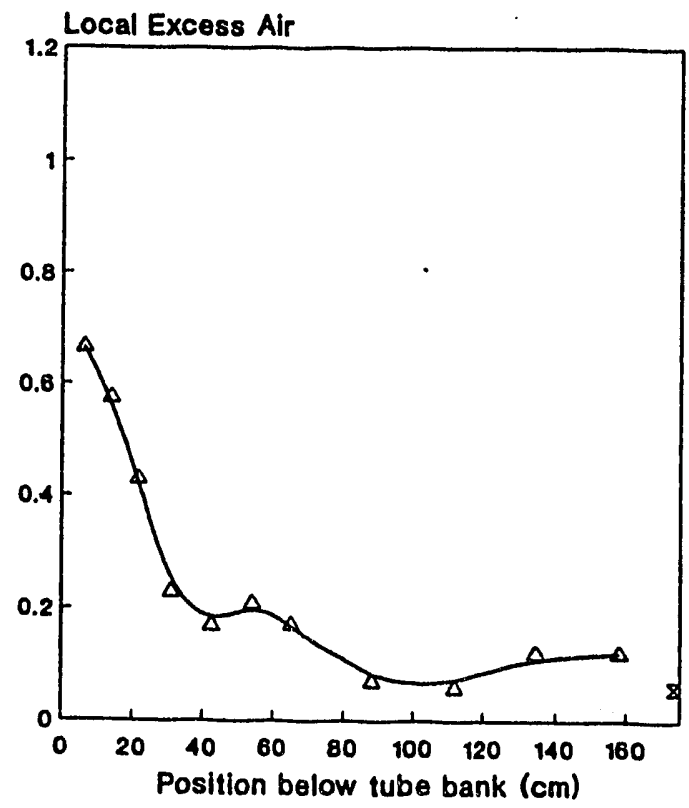

Draft: -.05 to -.23 inches water column

FIG. 7.10 


\section{PSD: Rosin-Rammler Plots}
Run: $\quad 8-13-91$
Coal 3
$E X o=5.7 \%$
Firing Conditions:
$12.0 \mathrm{lb} / \mathrm{hr}$
$30.8 \mathrm{scfm}$
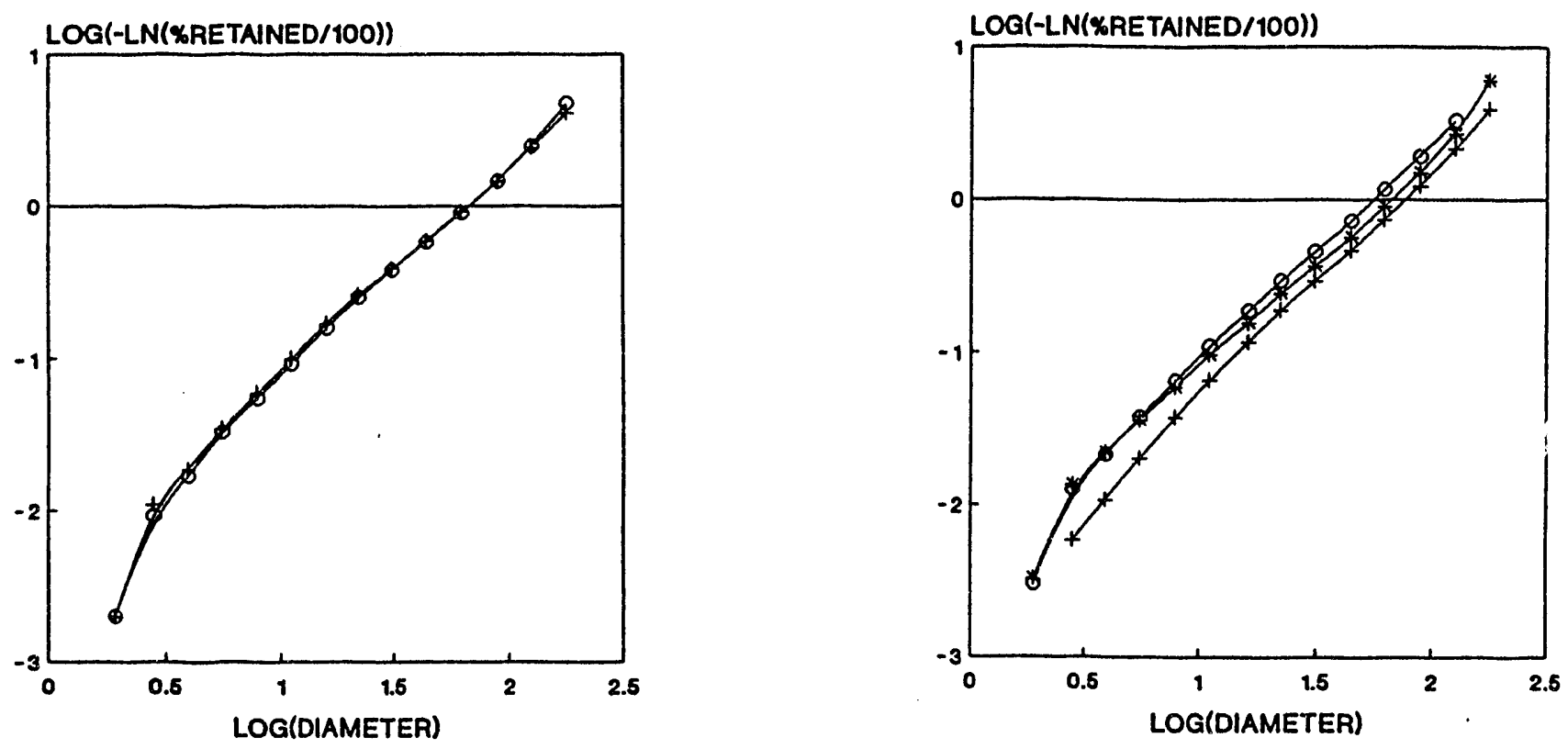

$0,+$ Feed

$0,4 . \cdot$ Port 0
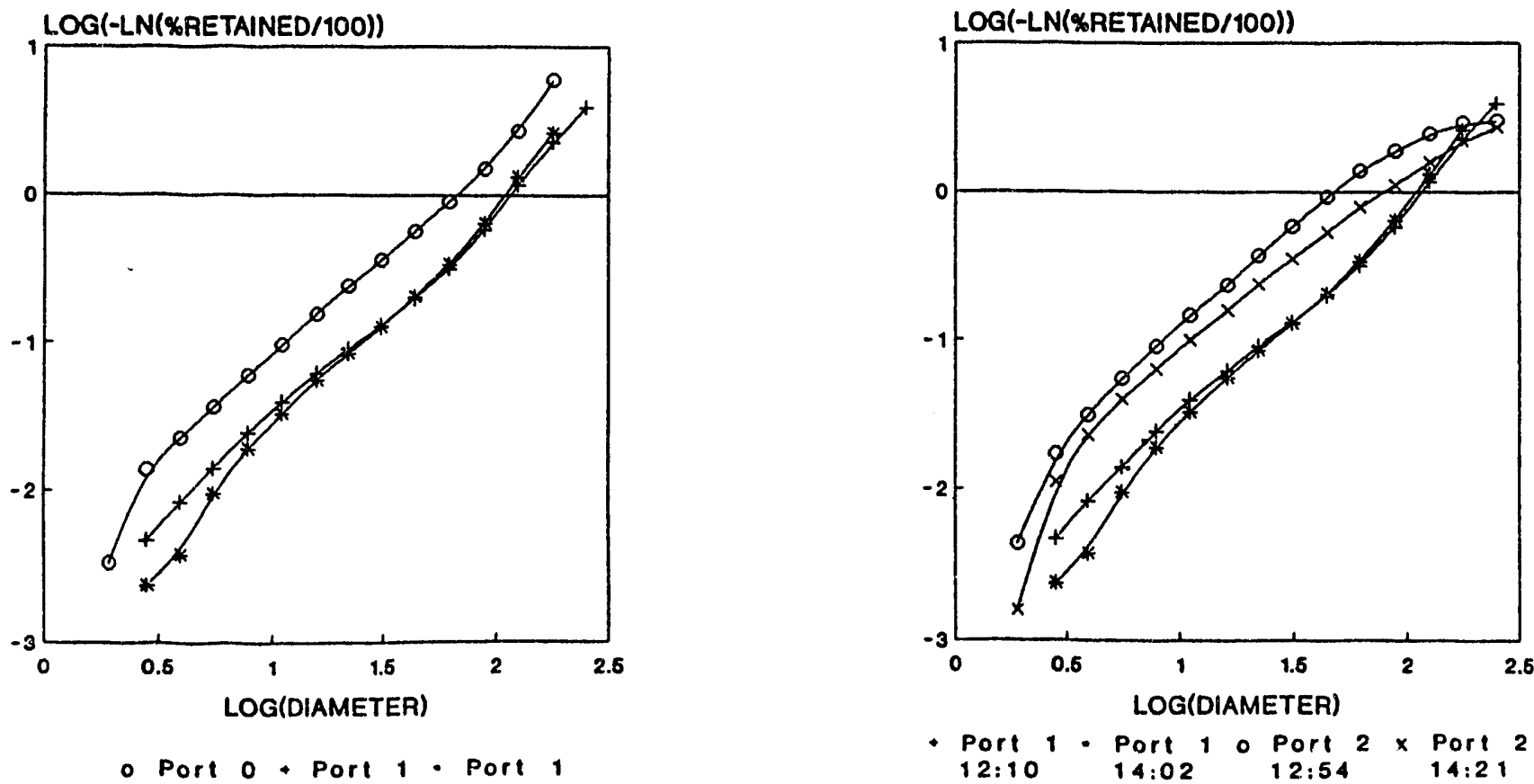

- Port O Port 1 Port 1

FIG. 7.11 


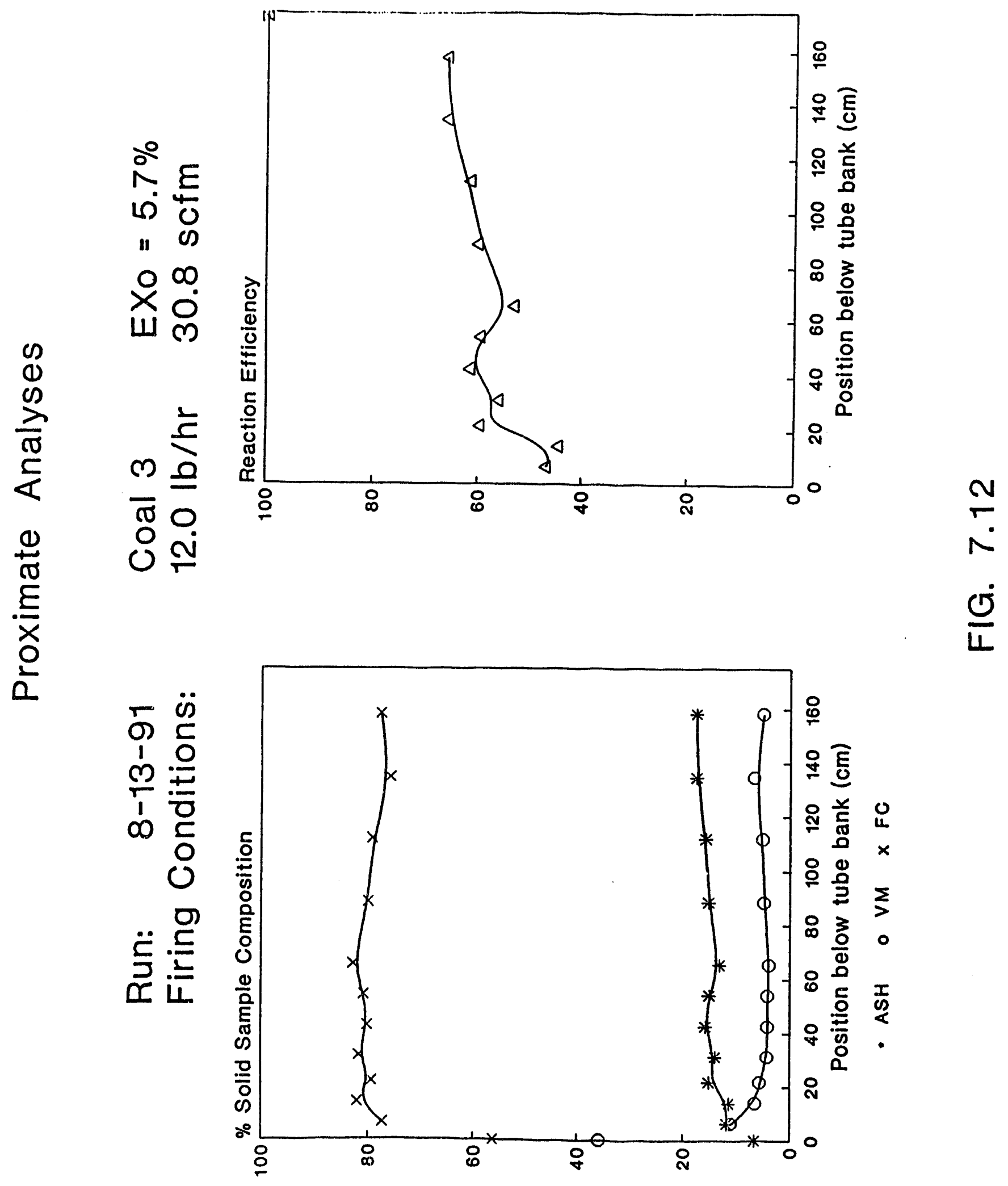




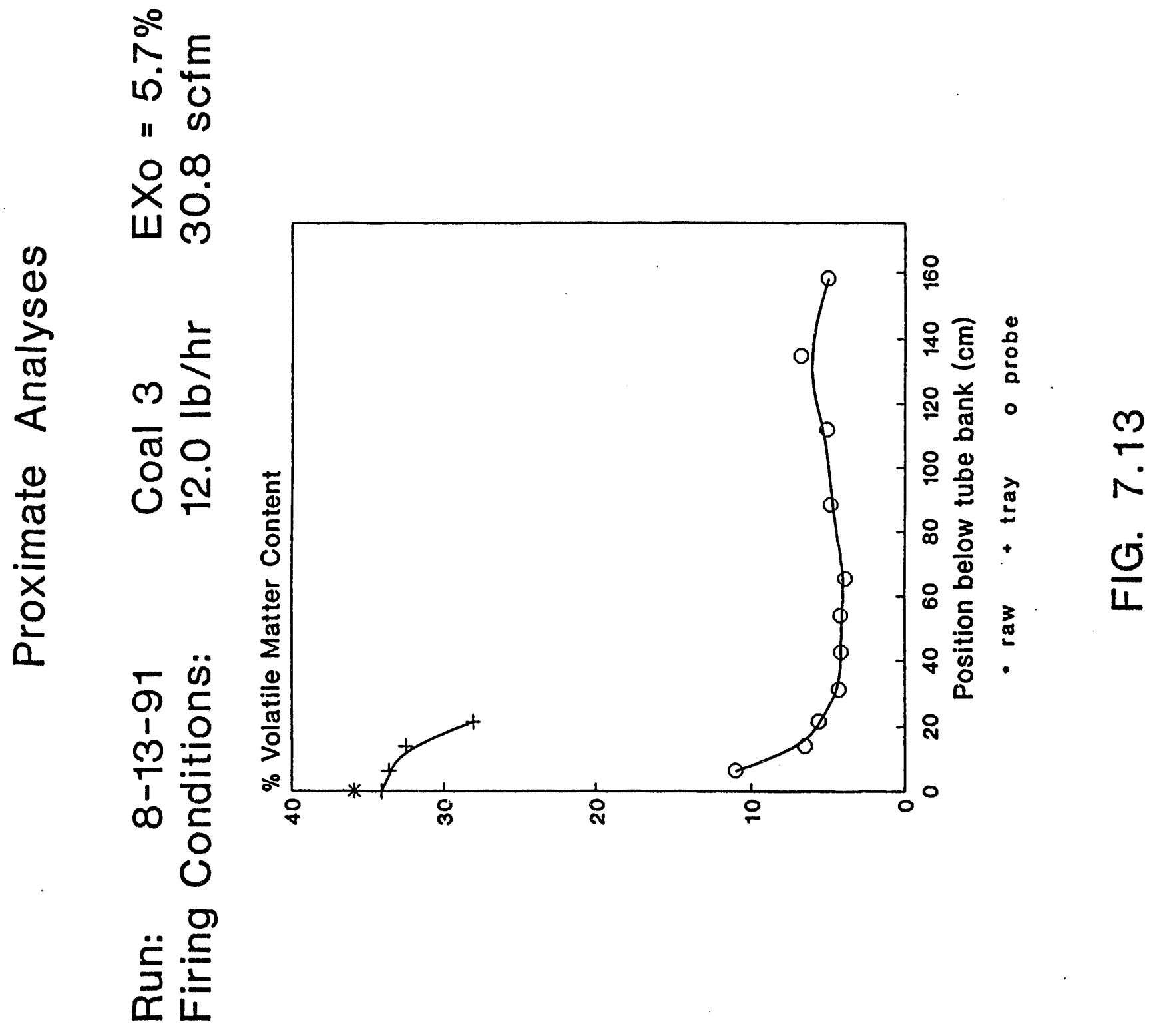



Run: $\quad 8-19-91$
Coal 3
$E X o=4.6 \%$
Firing Conditions:
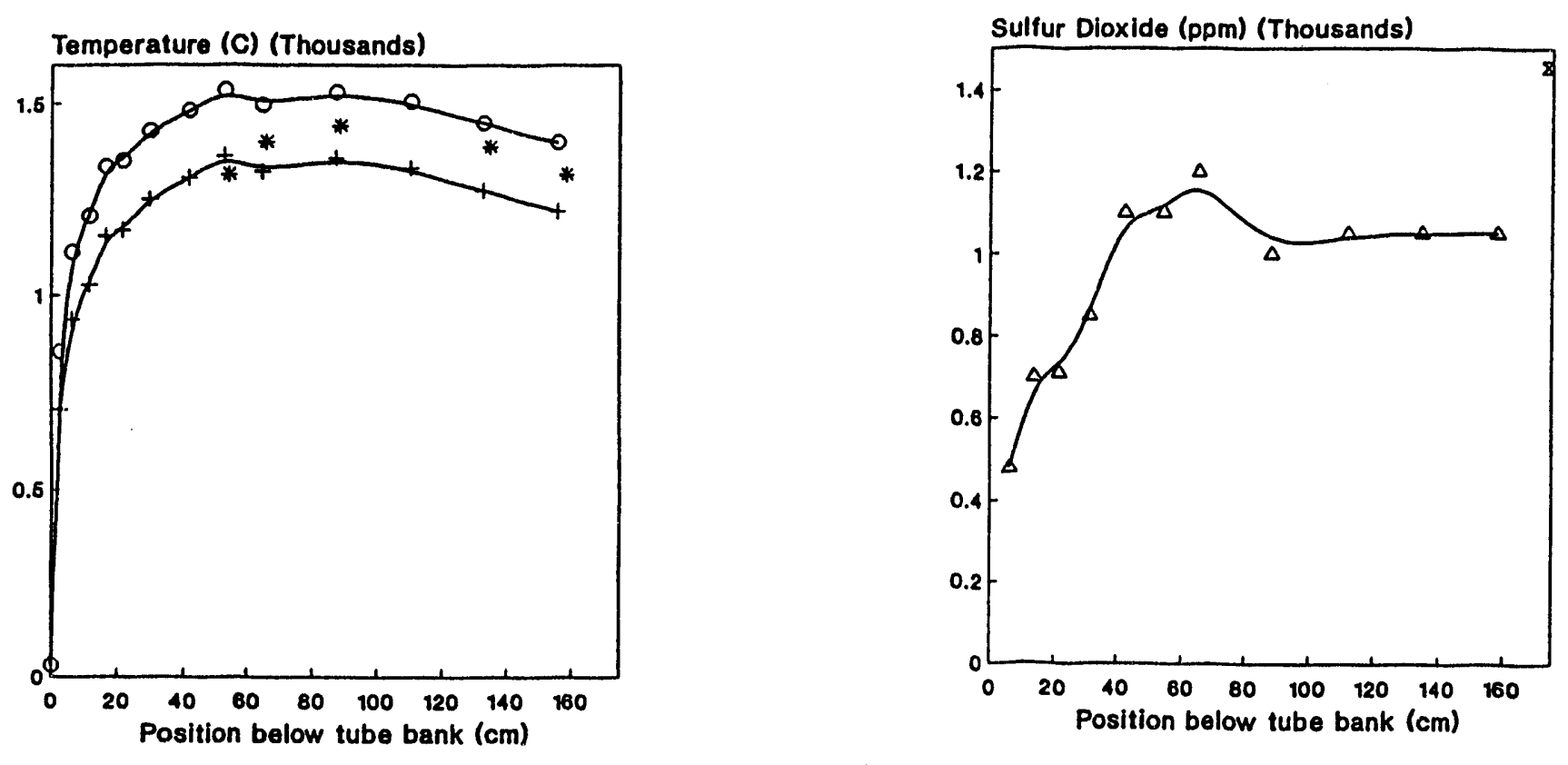

- Wall T Flame $\mathbf{T}$ o Calculated
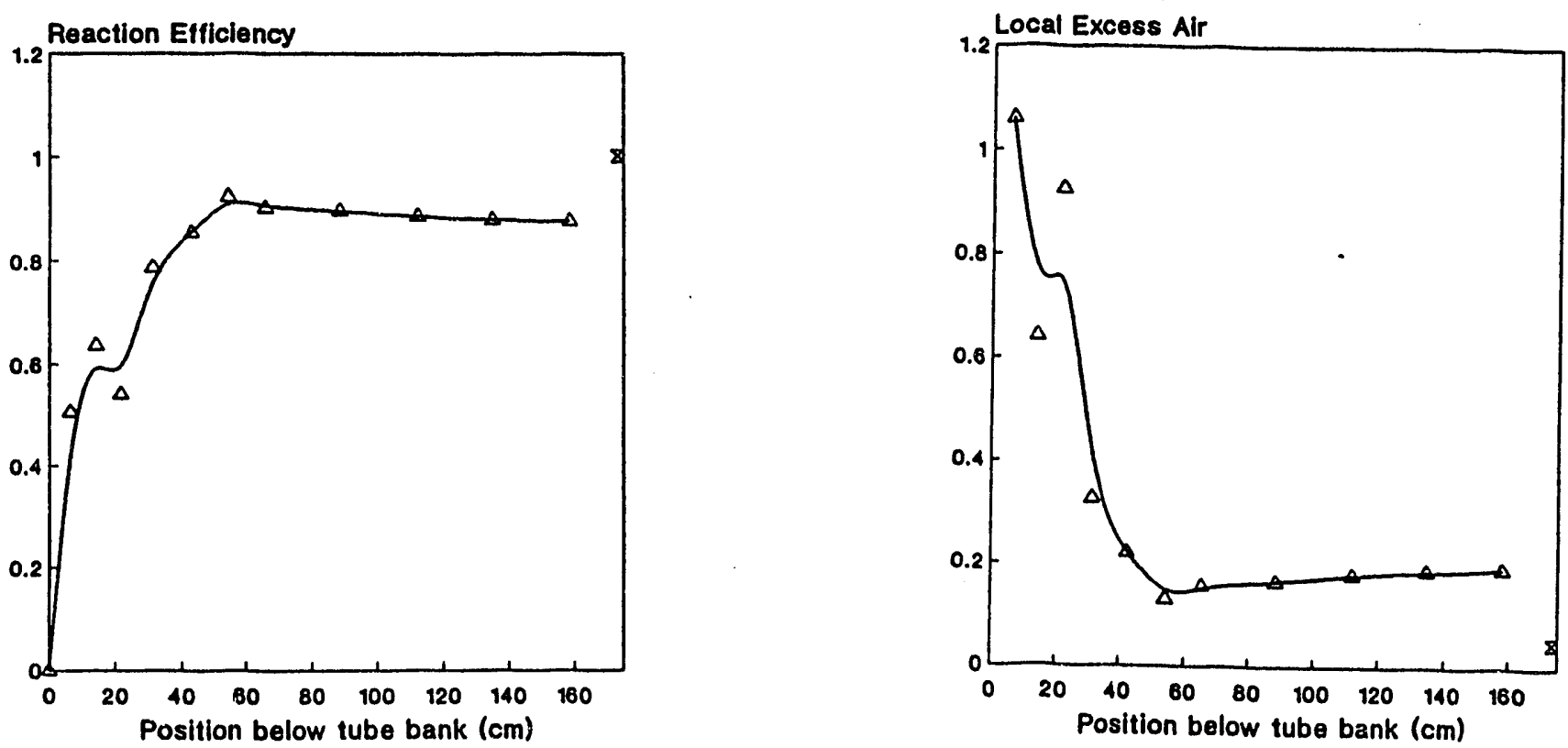

Draft: -.06 to -.26 inches water column

FIG. 7.14 


\section{PSD: Rosin-Rammler Plots}

$\begin{array}{llll}\text { Run: } & 8-19-91 & \text { Coal } 3 & \text { EXo }=4.6 \% \\ \text { Firing Conditions: } & 12.0 \mathrm{ib} / \mathrm{hr} & 30.7 \mathrm{scfm}\end{array}$

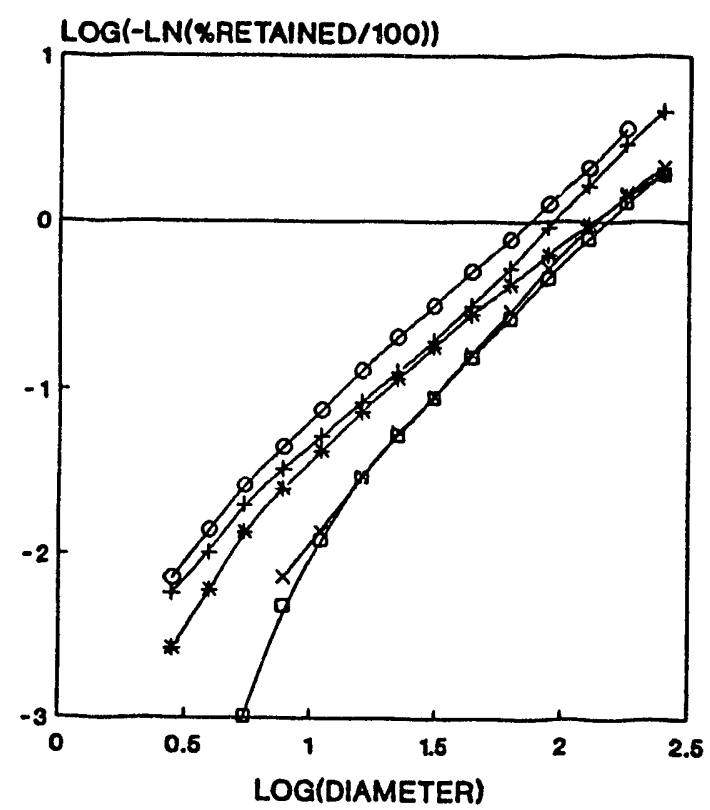

- Port 0 - Port 1 - Port 2 x Port 3 a Port 4
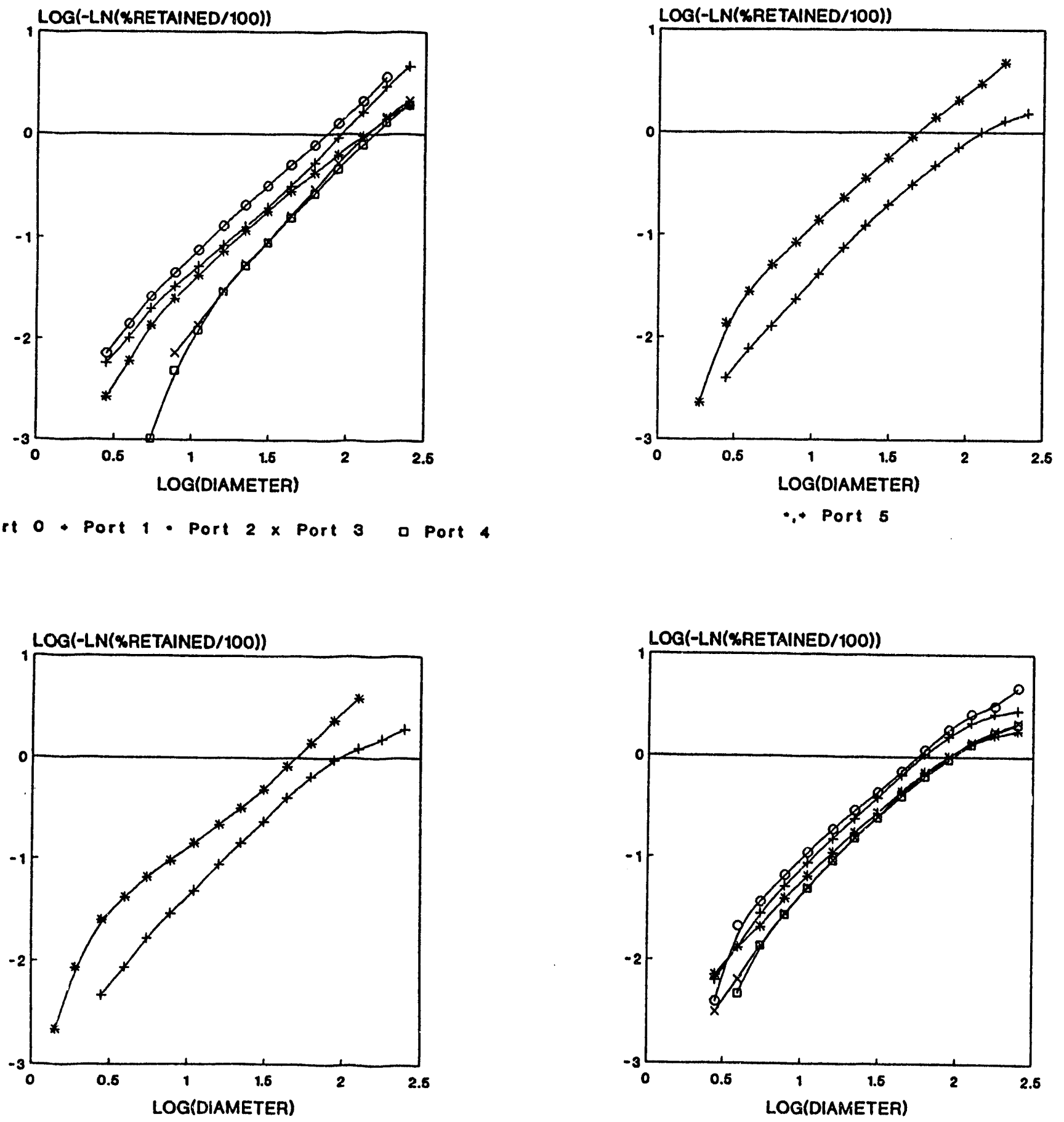

$\because$ Port 6

- Port 7 . Port 8 . Port $9 \times$ Port 10 D Port

FIG. 7.15 


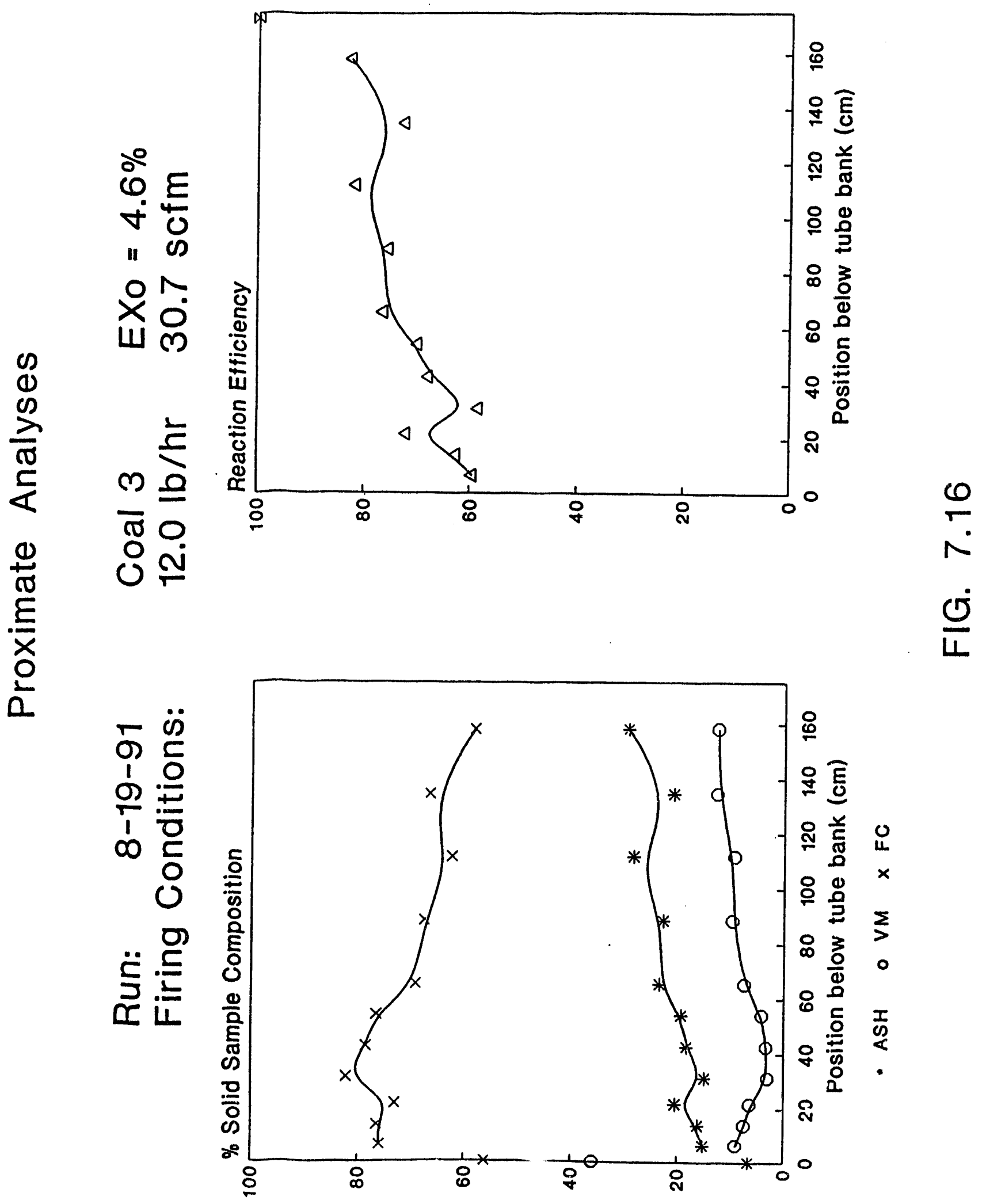


Run: $9-4-91 \quad$ Coal $4 \quad E X_{0}=-.7 \%$ Firing Conditions:

\section{$14.6 \mathrm{lb} / \mathrm{hr} \quad 34.8 \mathrm{scfm}$}
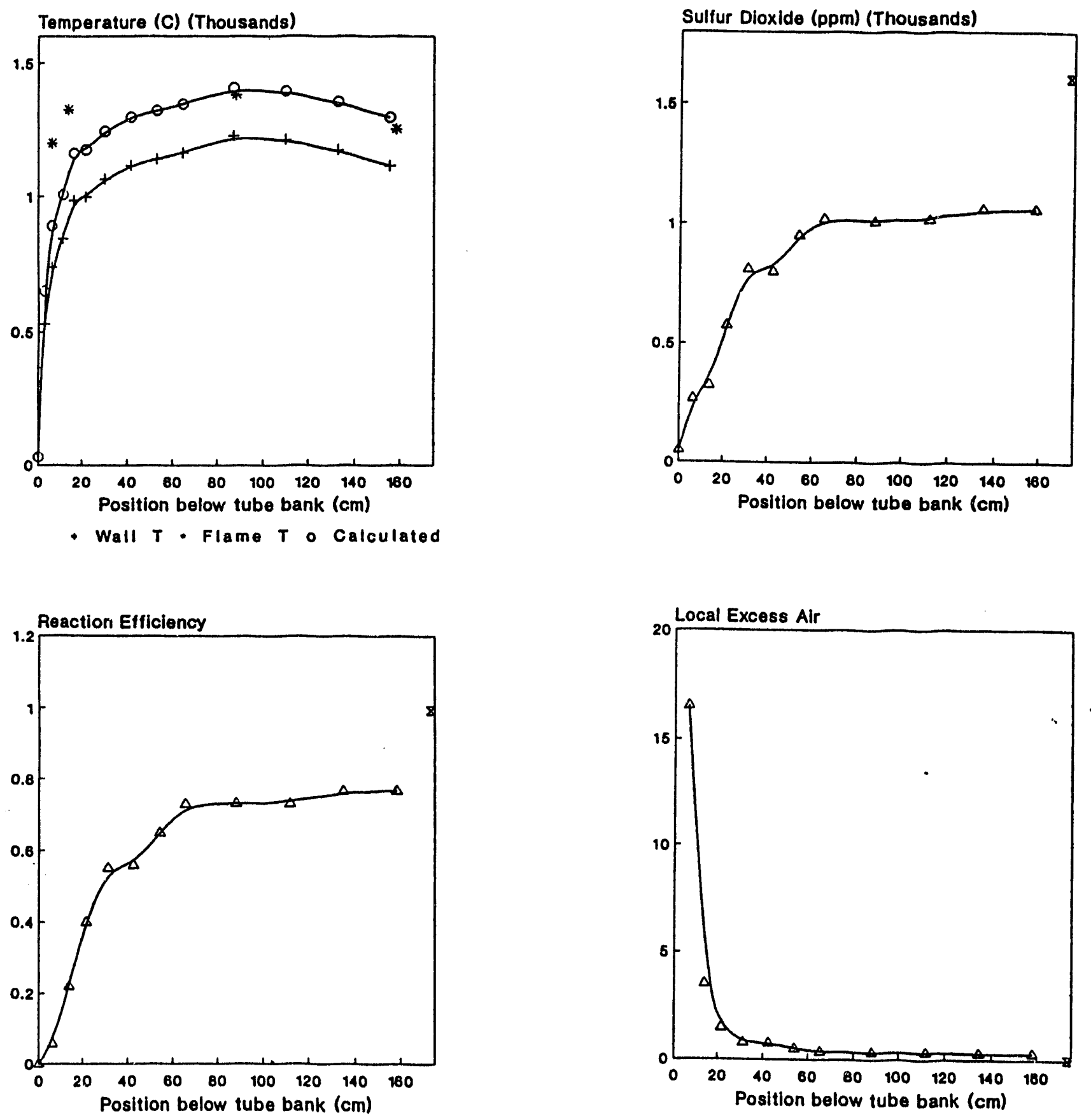

Draft: -.03 to -.43 inches water column

FIG. 7.17 


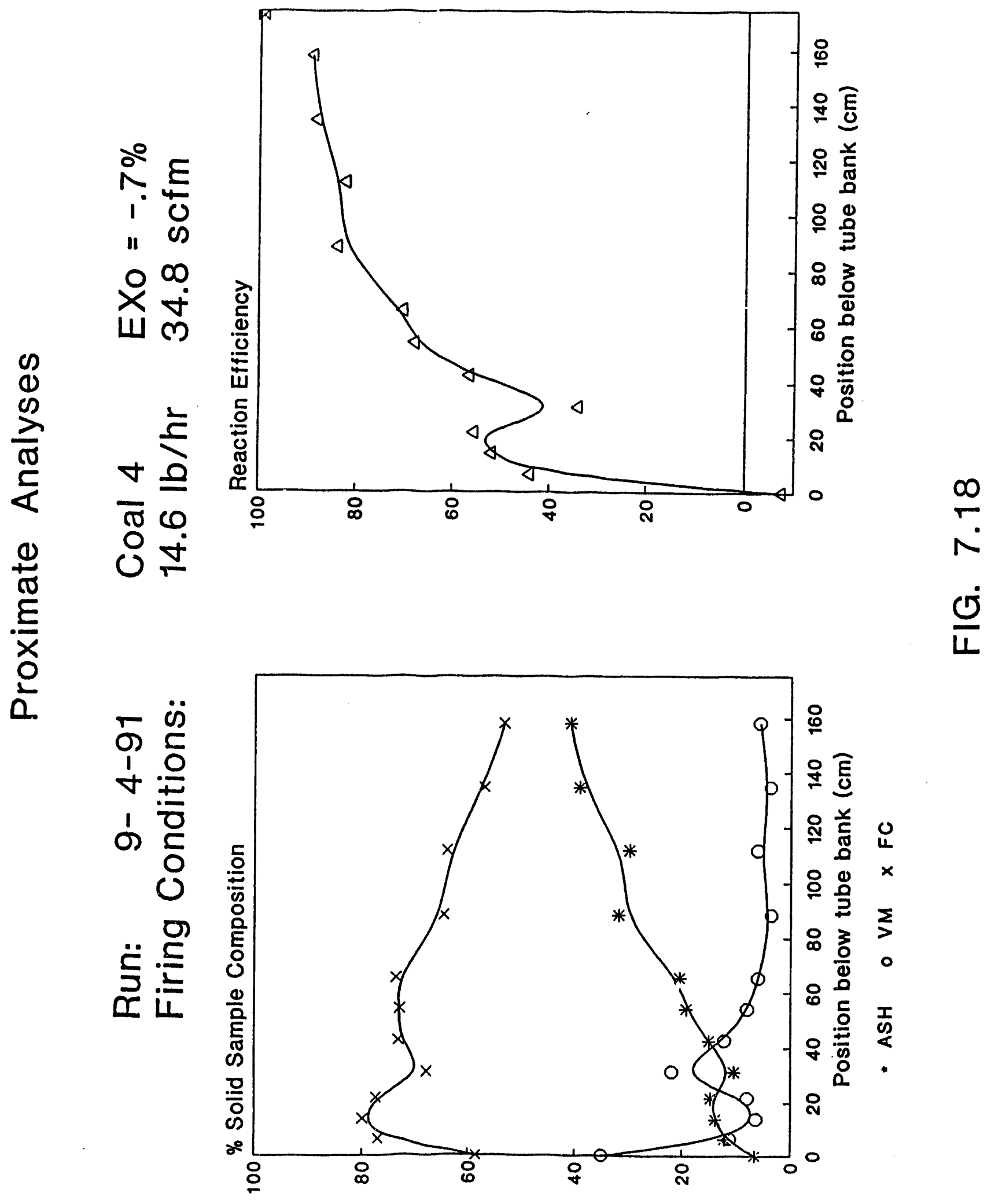


Run: $\quad 9-9-91$

Coal $9 \quad E X 0=-.6 \%$

Firing Conditions:

$15.6 \mathrm{lb} / \mathrm{hr} \quad 26.3 \mathrm{scfm}$
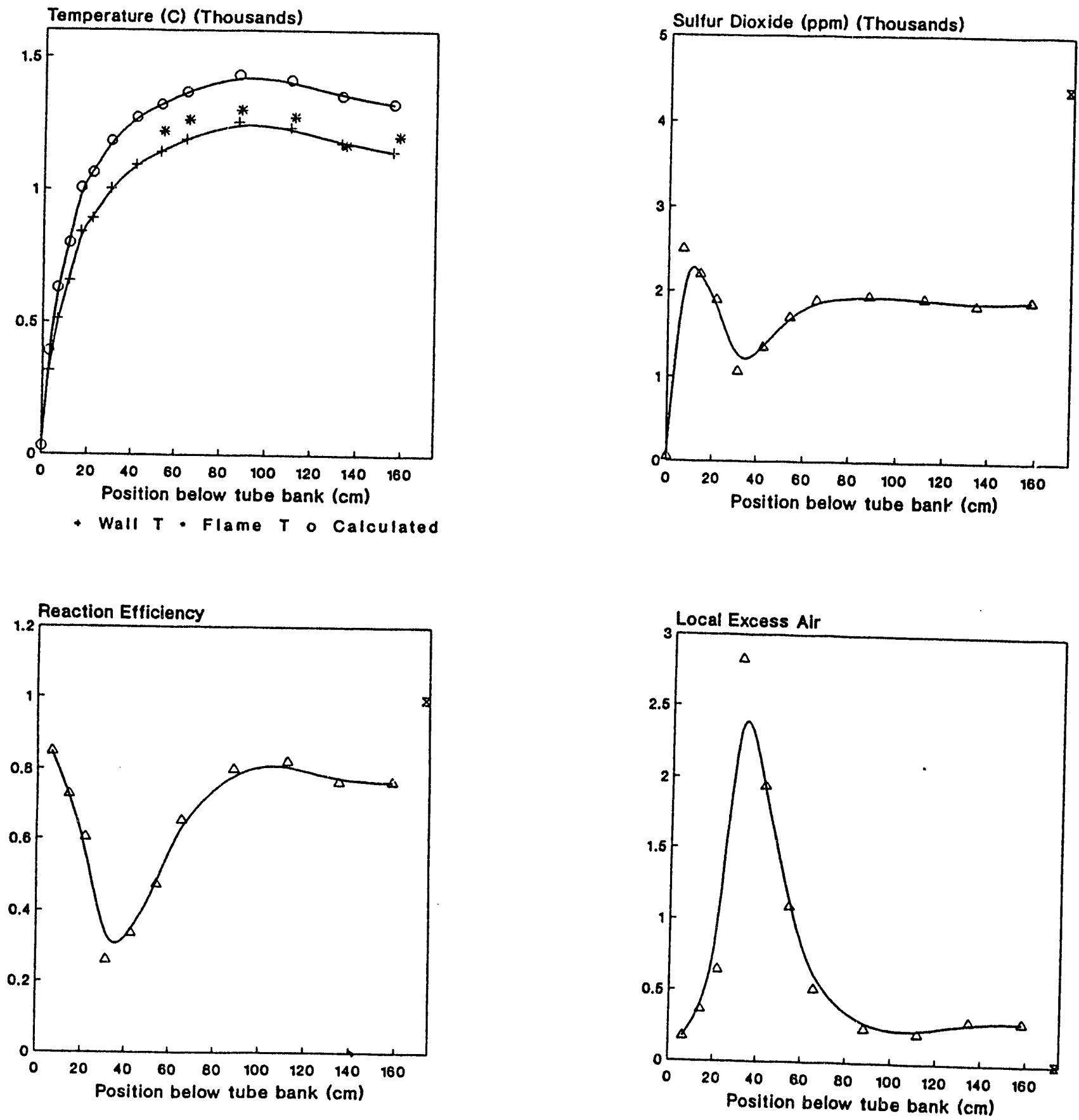

Draft: -.04 to -.21 inches water column

FIG. 7.19 


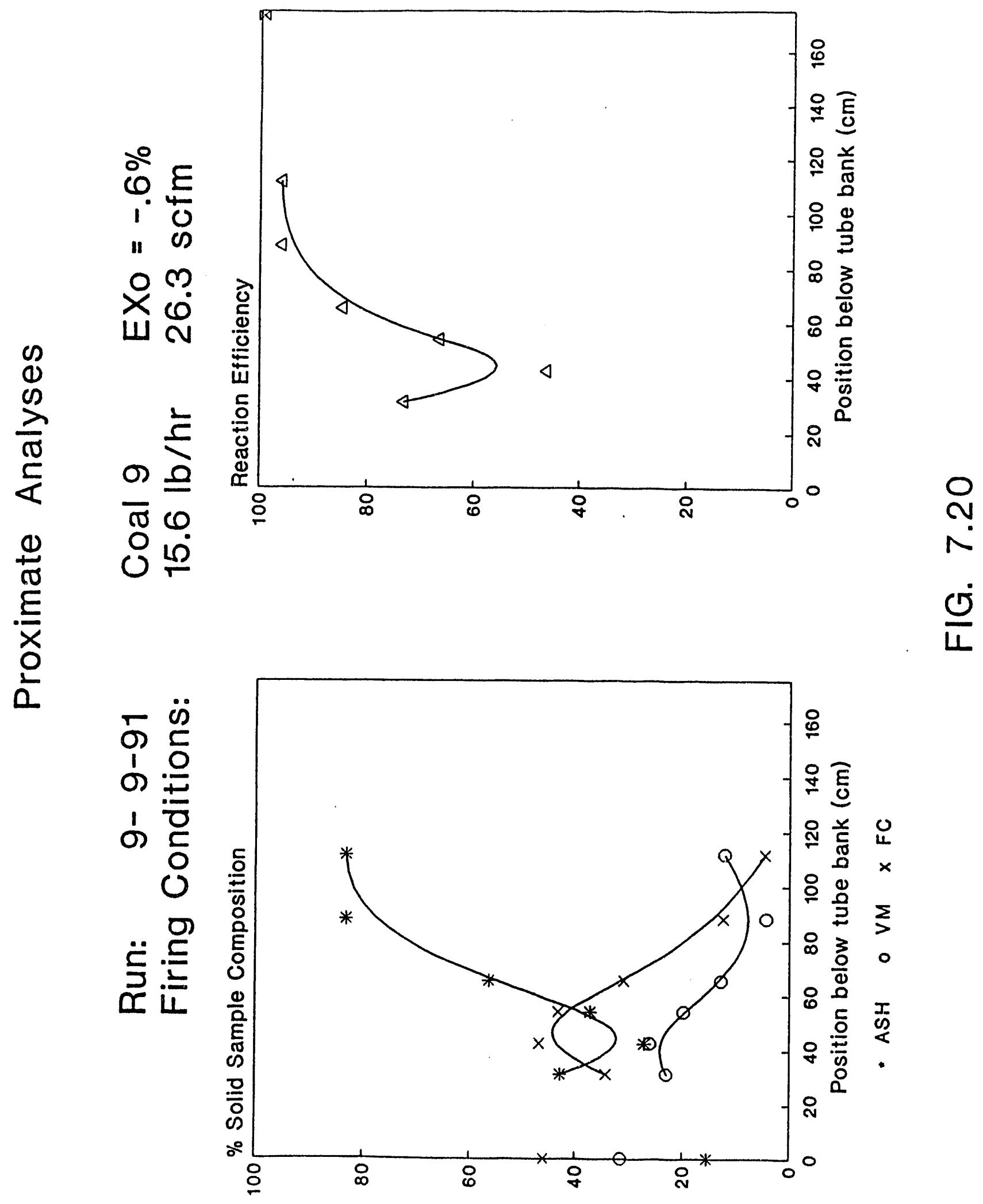




\section{Run: $9-9-91$ Coal 9 sorbent $E X o=-28.3 \%$ Firing Conditions: 18.1, $\quad 4.5 \mathrm{lb} / \mathrm{hr} 26.3 \mathrm{scfm}$}
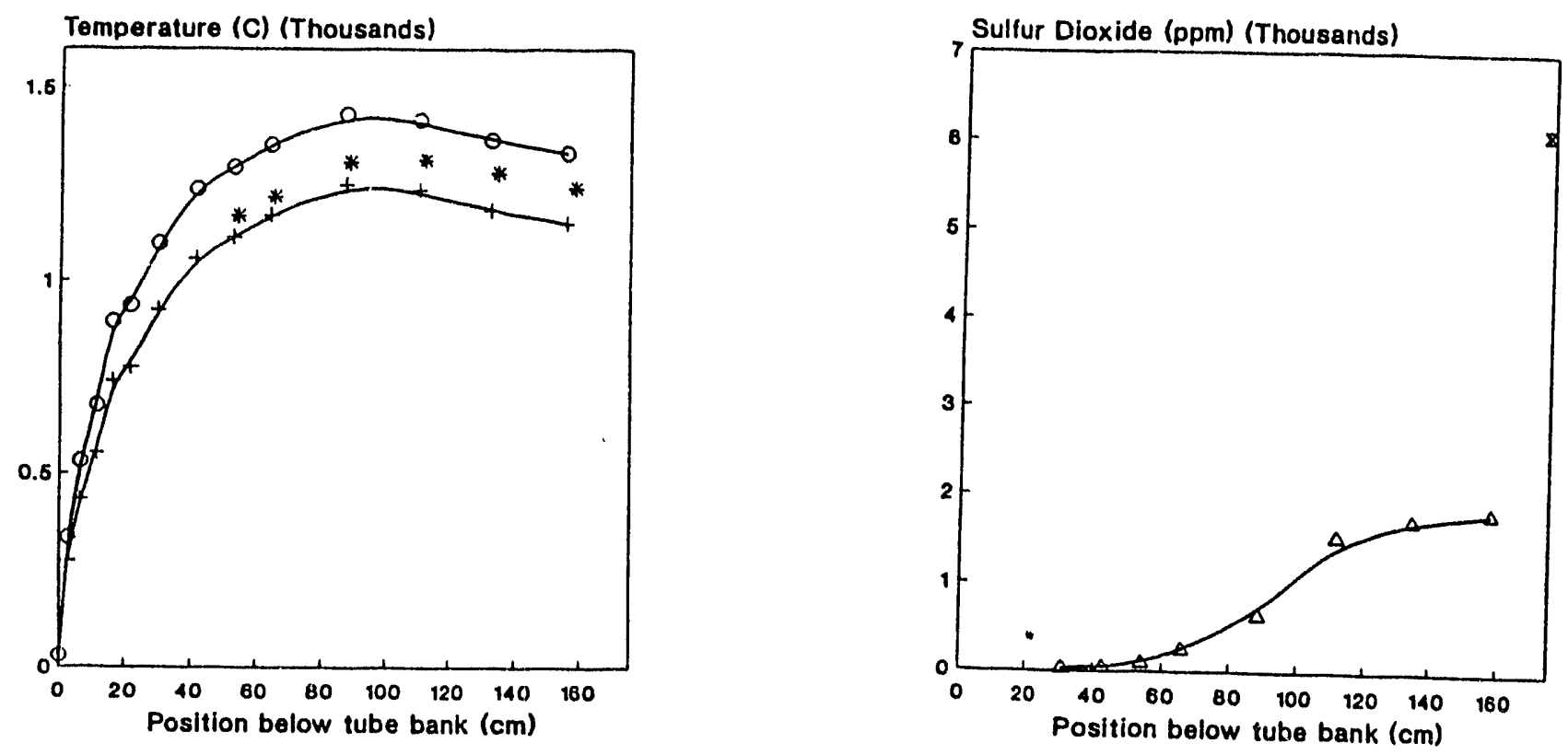

- Wall T - Flame T o Calculated
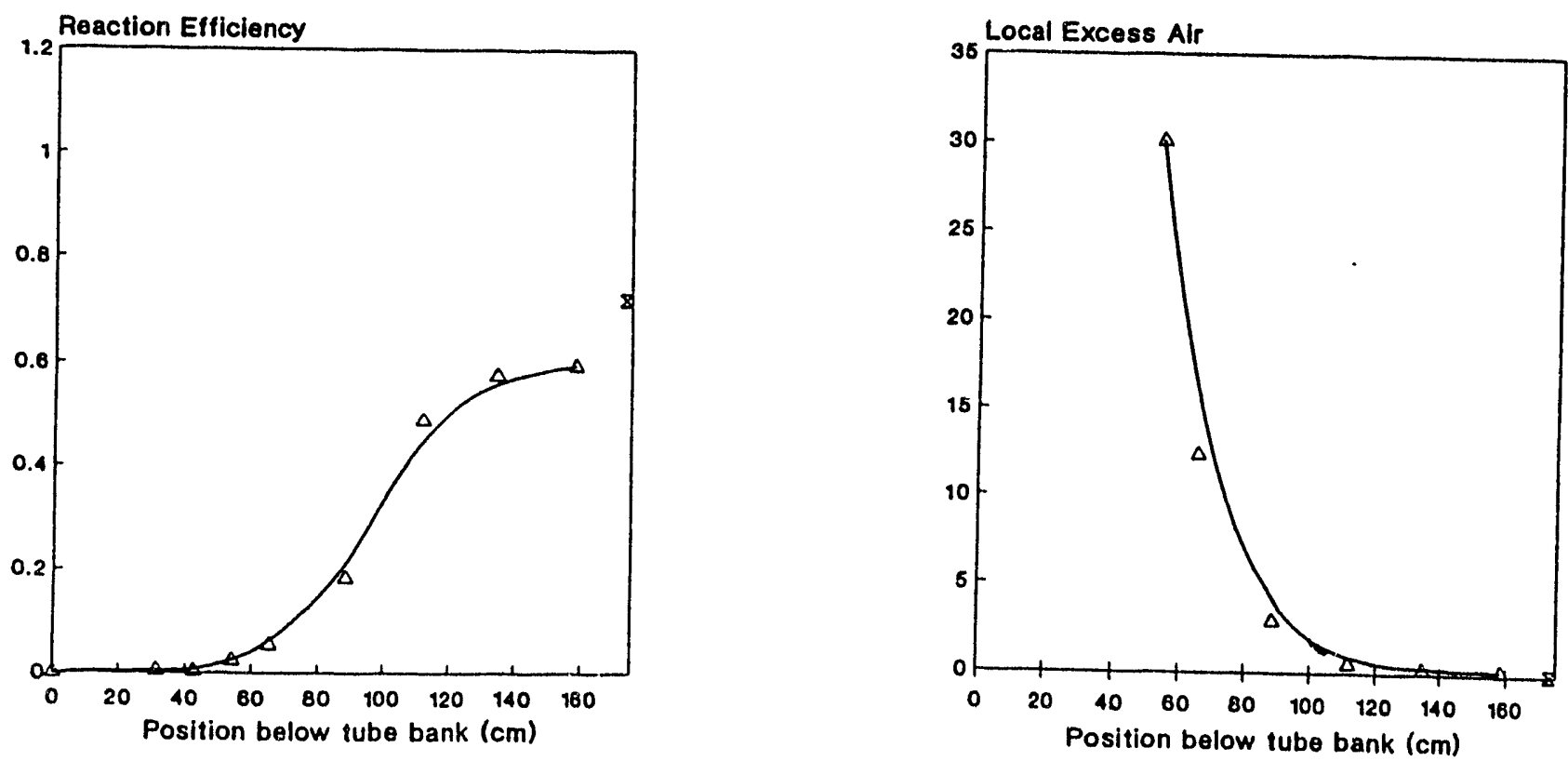

Draft: -.05 to -.19 inches water column

FIG. 7.21 


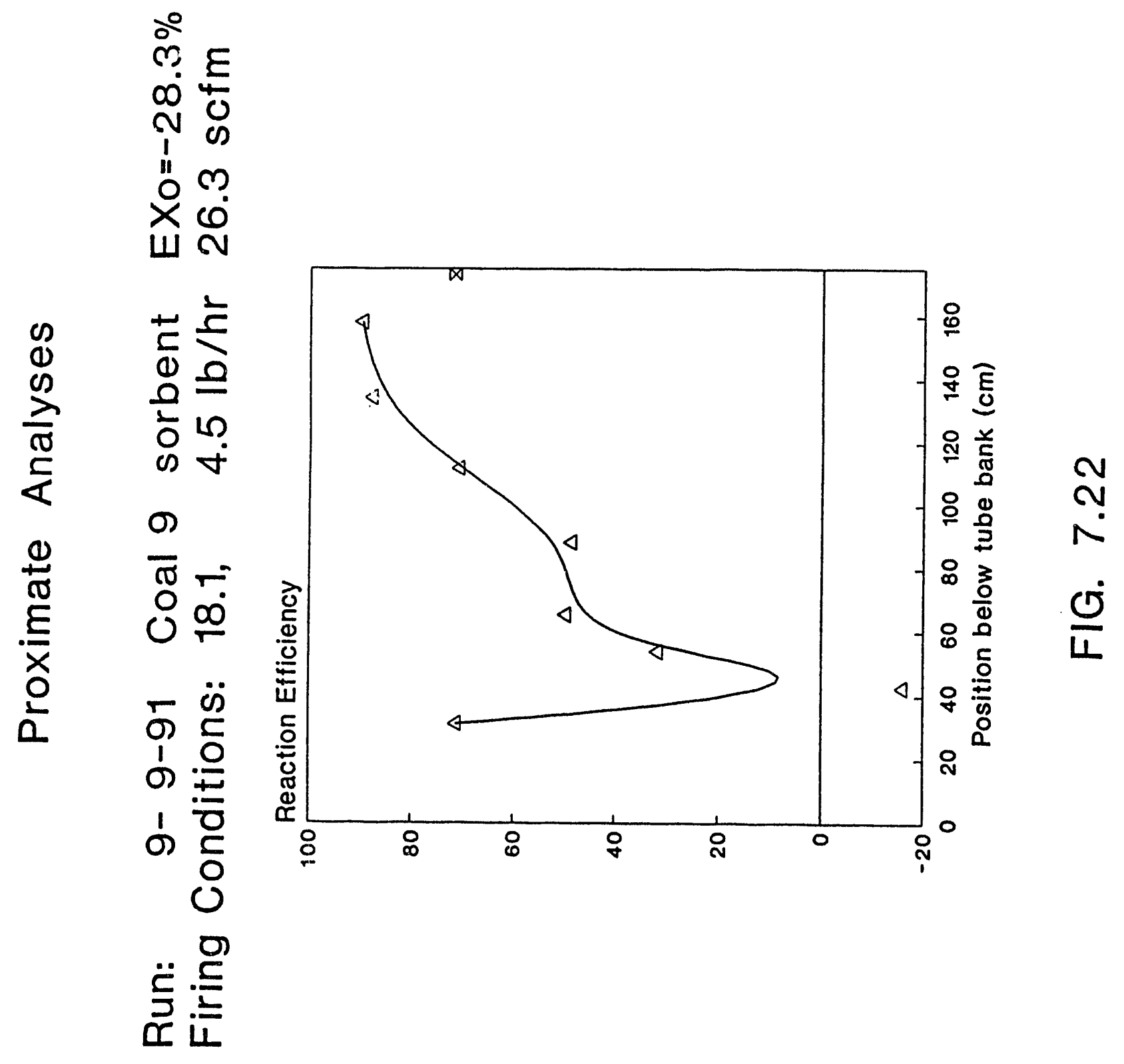


Run: $\quad 12-6-91$ Firing Conditions:

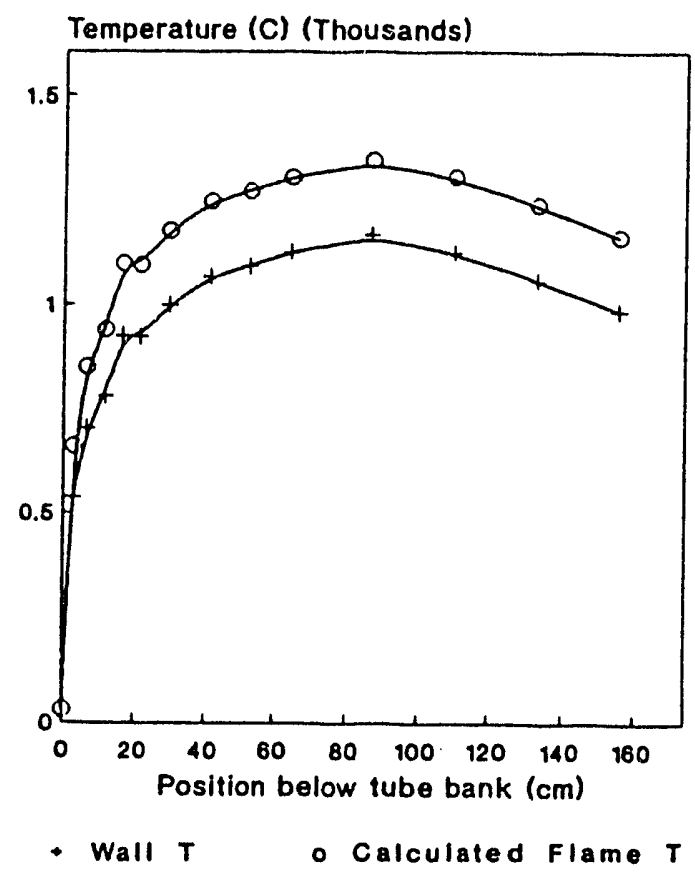

Coal $11 \quad E X O=-.5 \%$ $9.4 \mathrm{lb} / \mathrm{hr} \quad 21.0 \mathrm{scfm}$

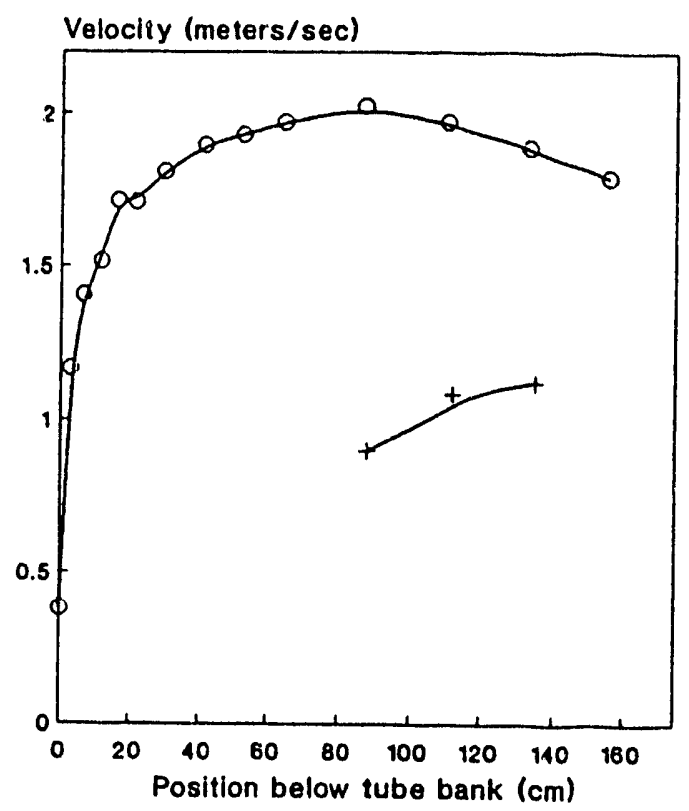

- Calculated oas veloclty

- Measured partlcle velocity

HORIZONTAL PROFILE AT PORT 9

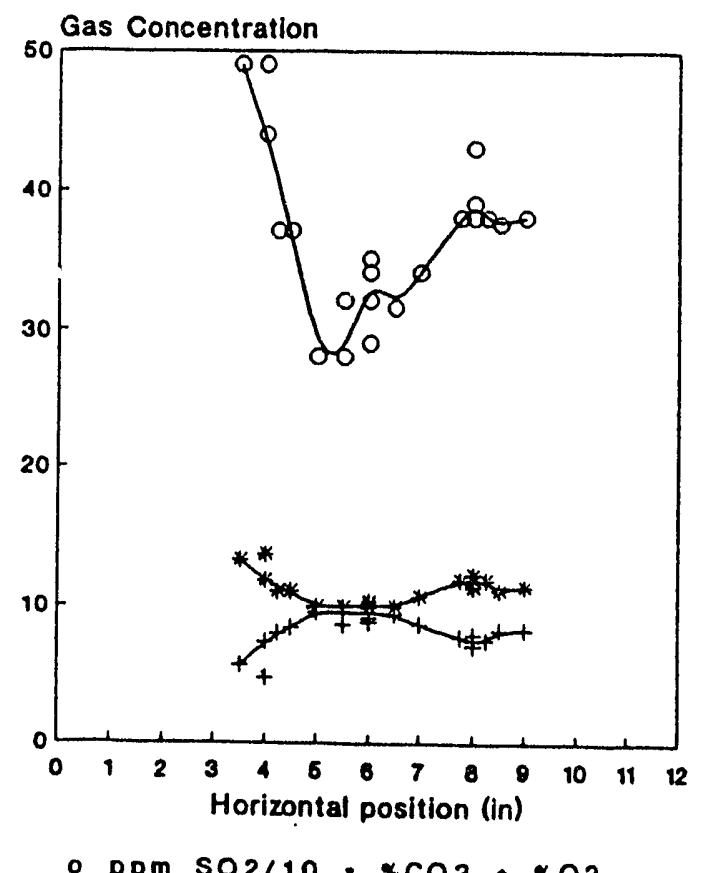

- ppm SO2/10.xcO2. \%O2

FIG. 7.23 


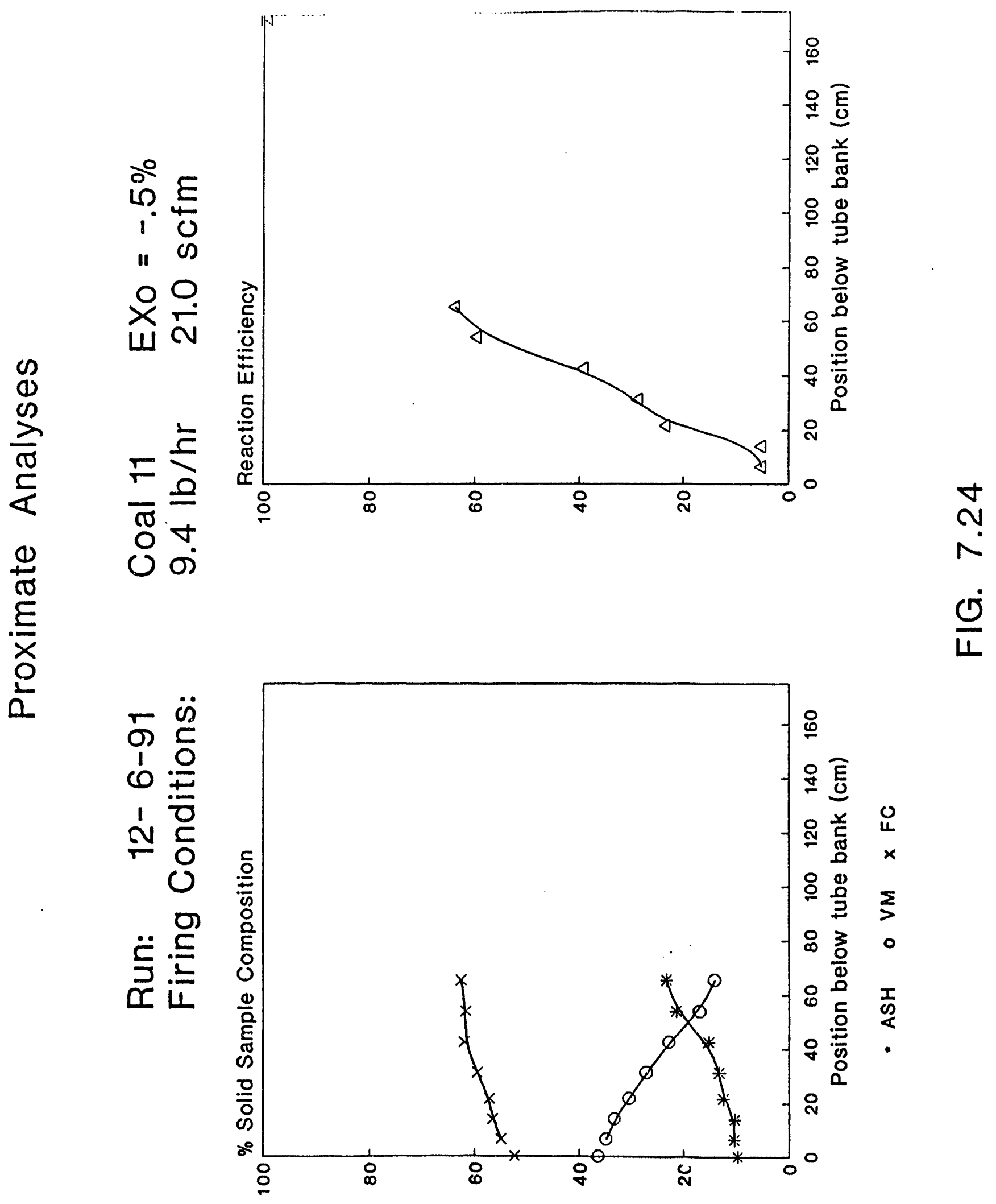



Run: $\quad 12-12-91$
Coal 11
$E X O=1.4 \%$
Firing Conditions:

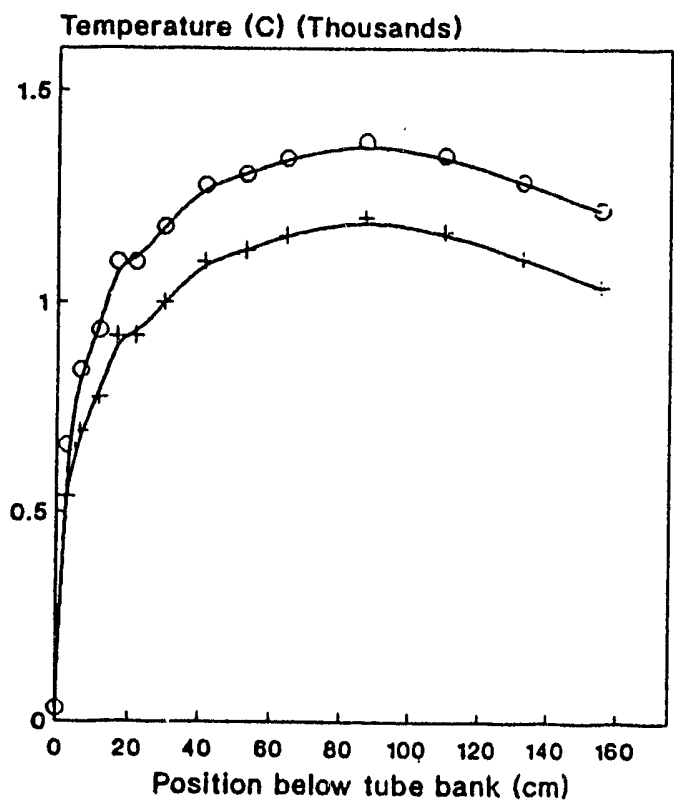

+ Wall T

- Calculated Flame T
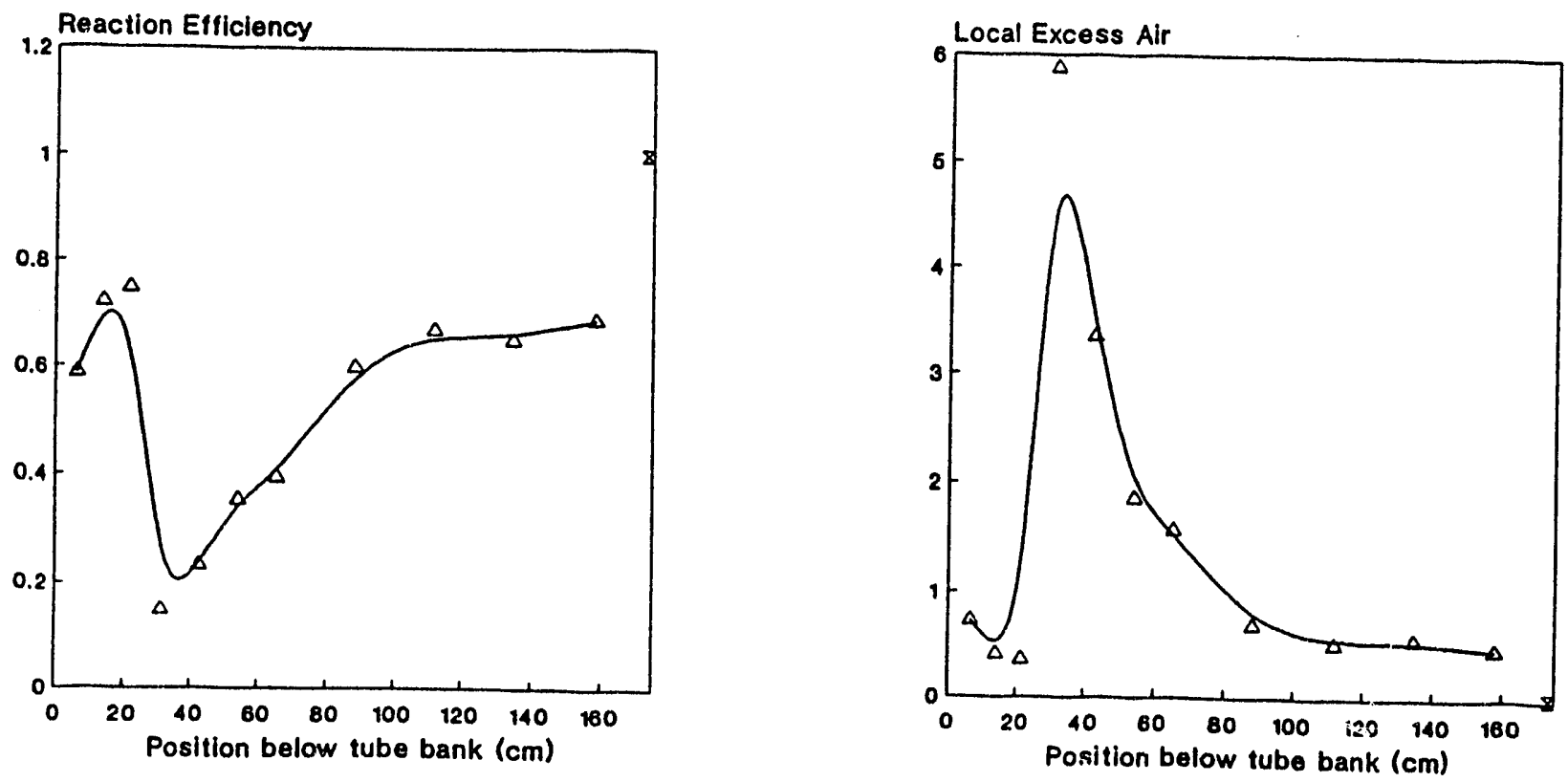

FIG. 7.25 

Run: $\quad 12-12-91$
Coal 11
$E X o=1.4 \%$
Firing Coryditions:
$9.5 \mathrm{lb} / \mathrm{hr}$
$22.0 \mathrm{scfm}$

HORIZONTAL PROFILE AT PORT 2

HORIZONTAL PROFILE AT PORT 4
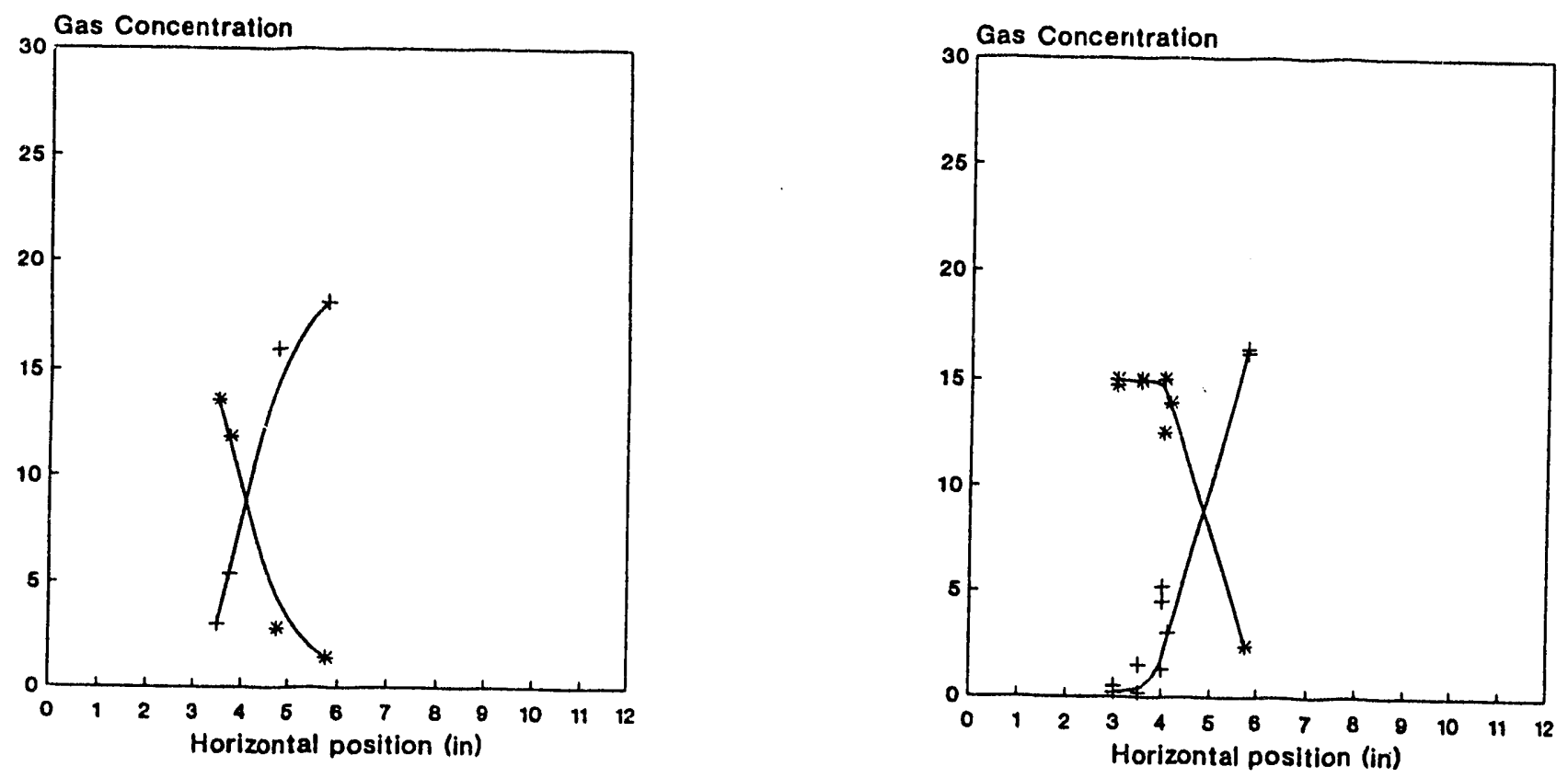

oppm s02110 * \%002 *02

- ppm s02/10 * xcO2+\%02

HORIZONTAL PROFILE AT PORT 9

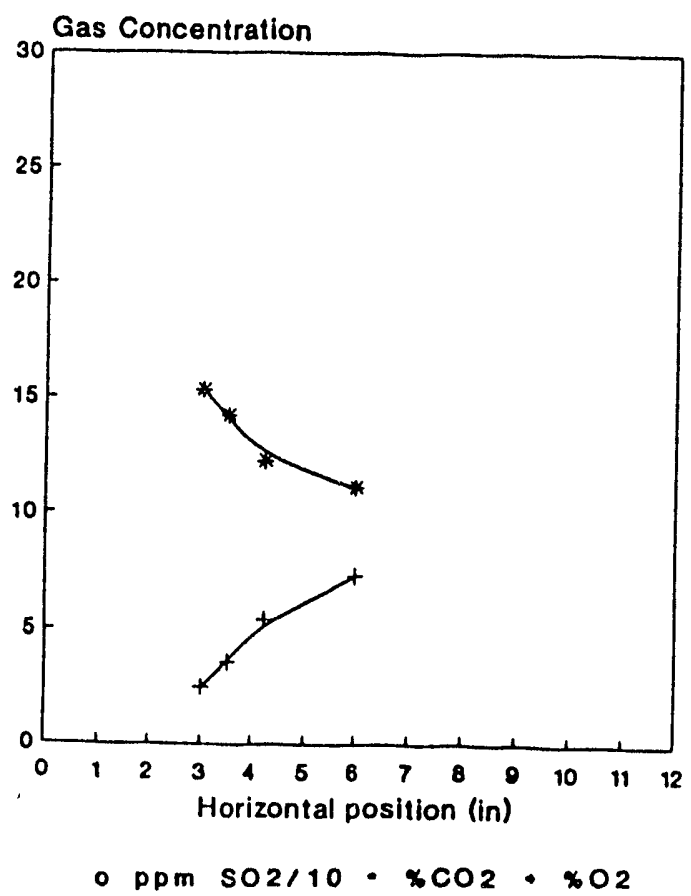

FIG. 7.26 


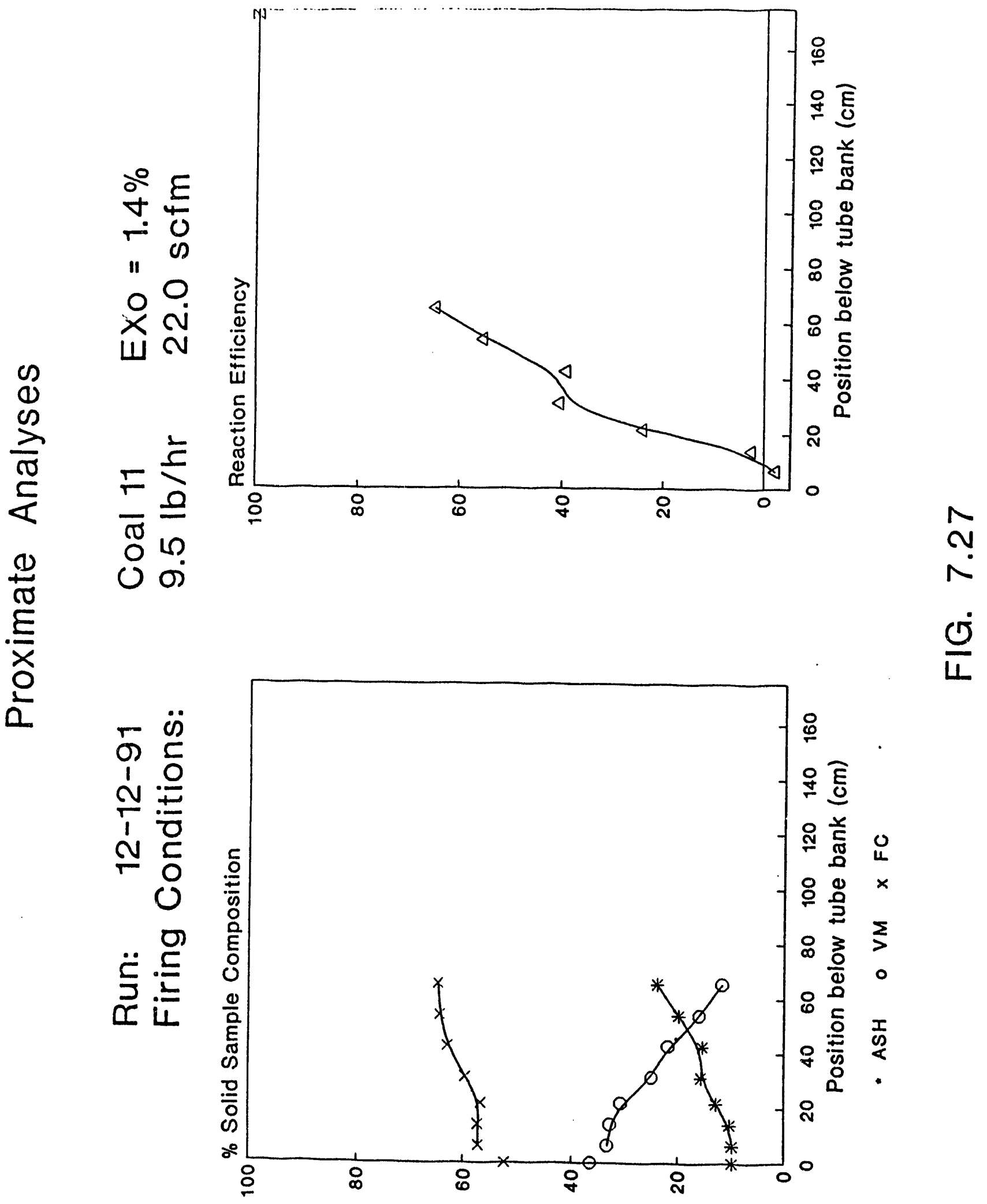




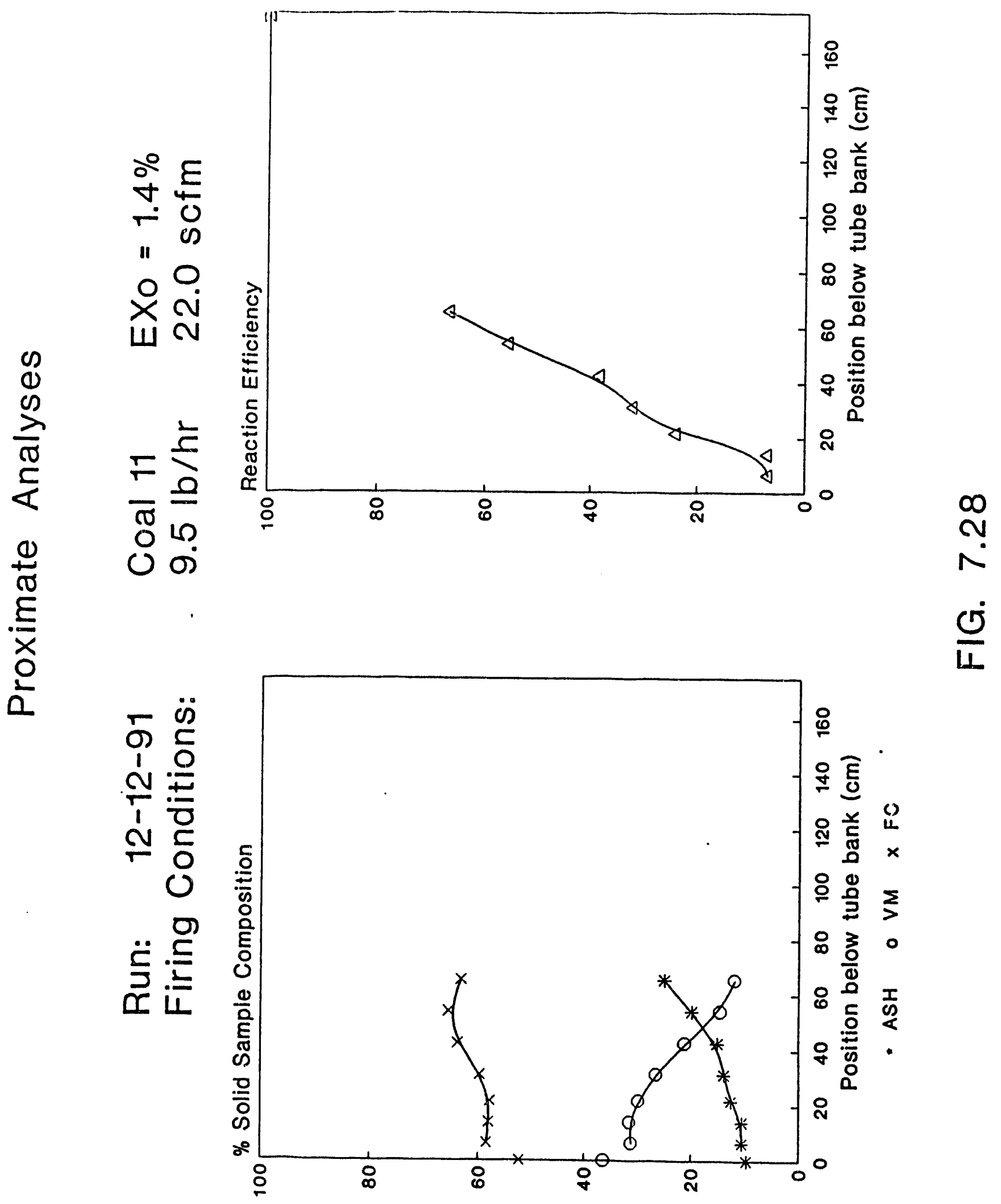


$\begin{array}{lclc}\text { Run: } & 12-17-91 & \text { Coal } 11 & \text { EXo }=-4.2 \% \\ \text { Firing Conditions: } & 9.5 \mathrm{lb} / \mathrm{hr} & 20.7 \mathrm{scfm}\end{array}$

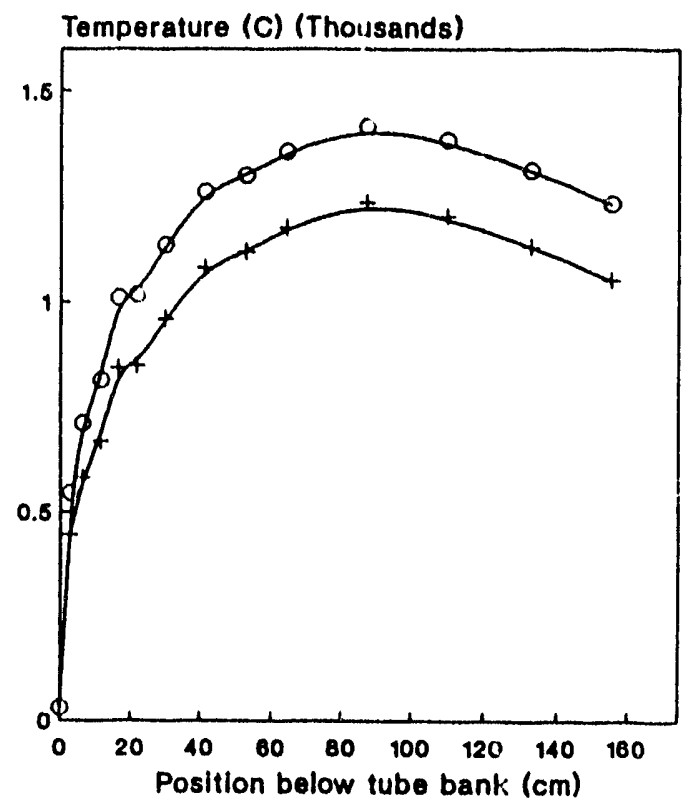

- Wall T

- Calculated flame T

HORIZONTAL PROFILE AT PORT 2

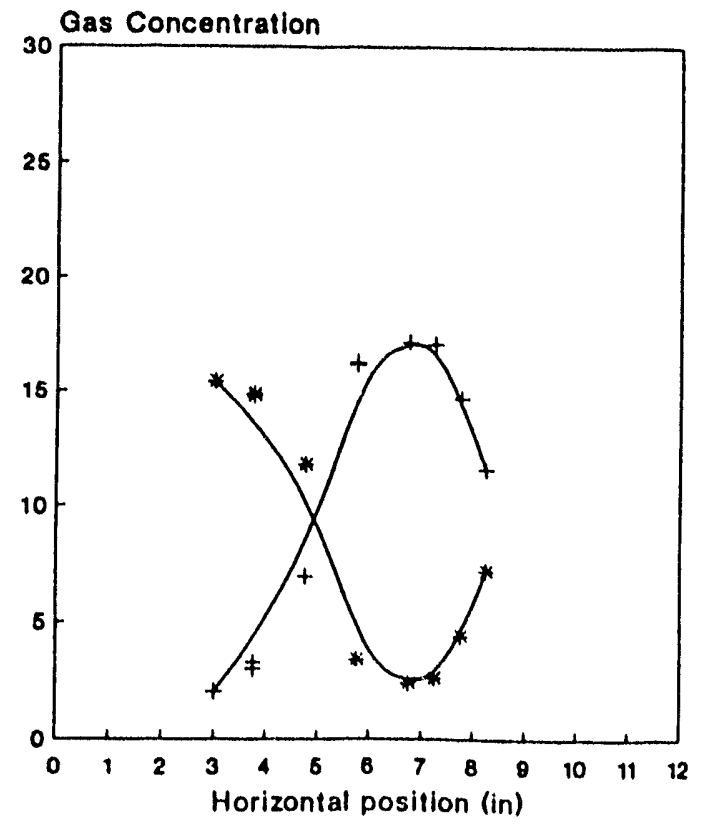

oppm SO2/10*xCO2*xO2
HORIZONTAL PROFILE AT PORT 2, INCREASED DRAFT

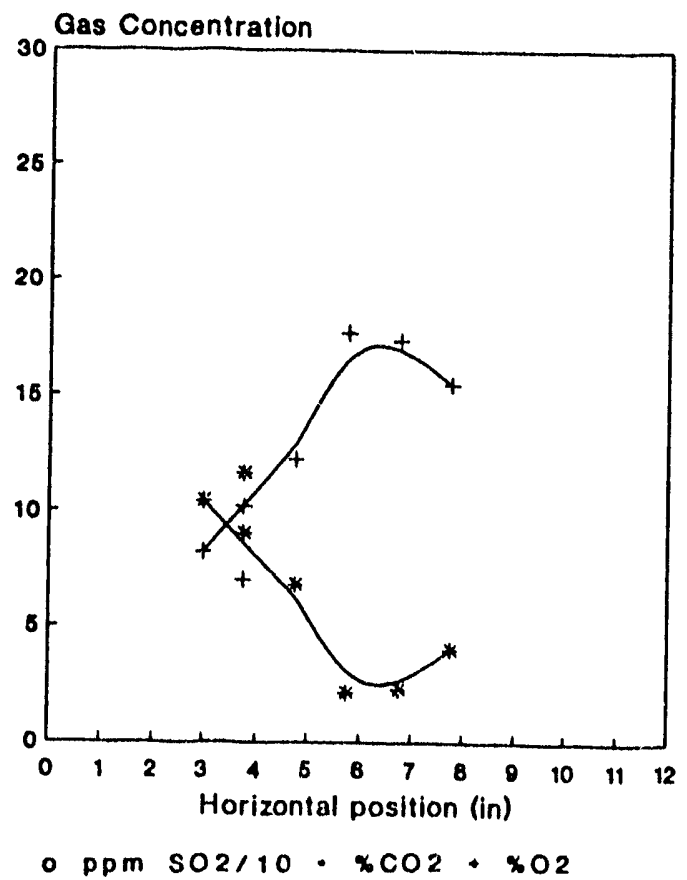

FIG. 7.29 
Run: 12-19-91 Firing Conditions:

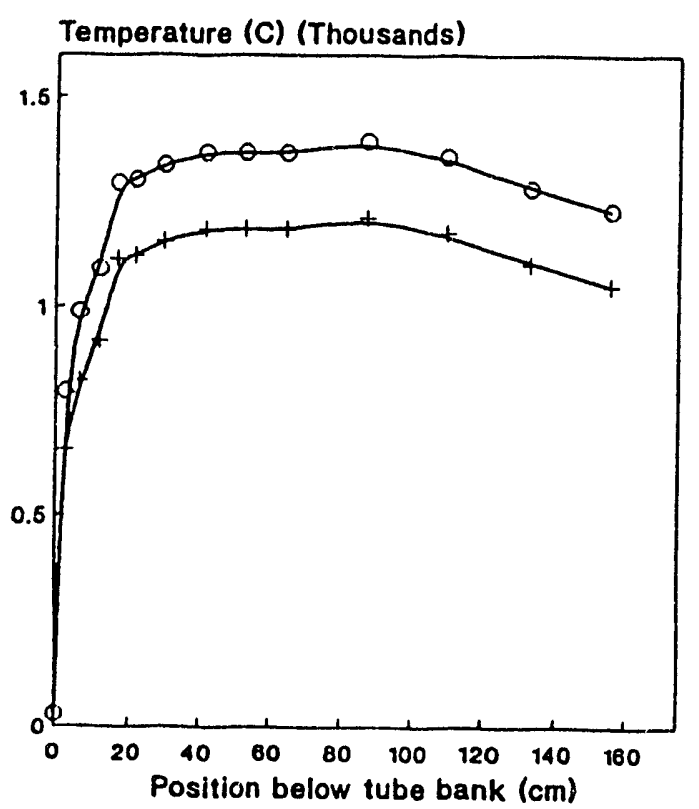

- Wall $T$ o Calculated Flame $T$
Coal $11 \quad E X O=-27.0 \%$

$11.2 \mathrm{lb} / \mathrm{hr} \quad 17.9 \mathrm{scfm}$

HORIZONTAL TEMPERATURE

PROFILE AT PORT 2

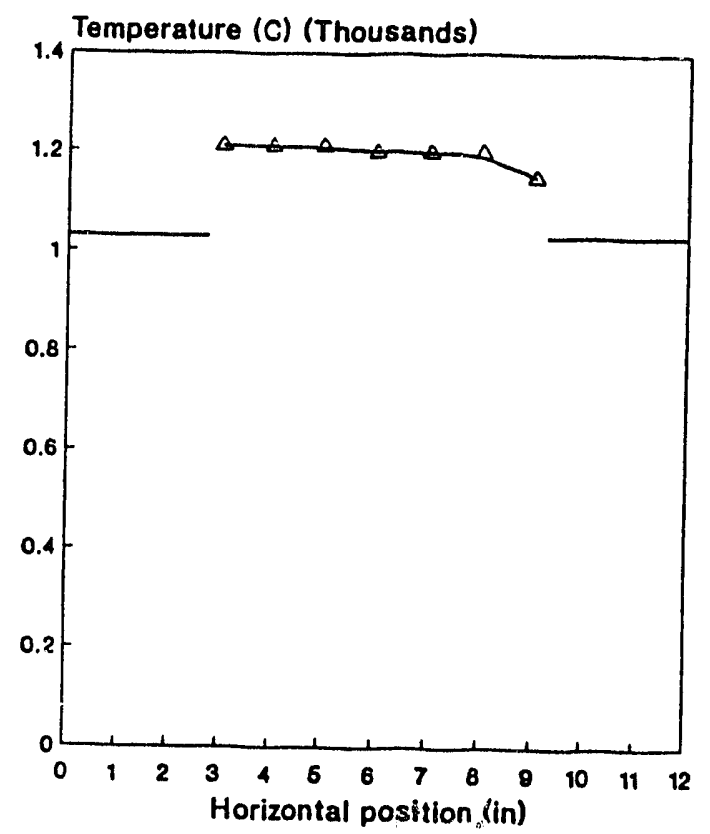

HORIZONTAL PROFILE AT PORT 2

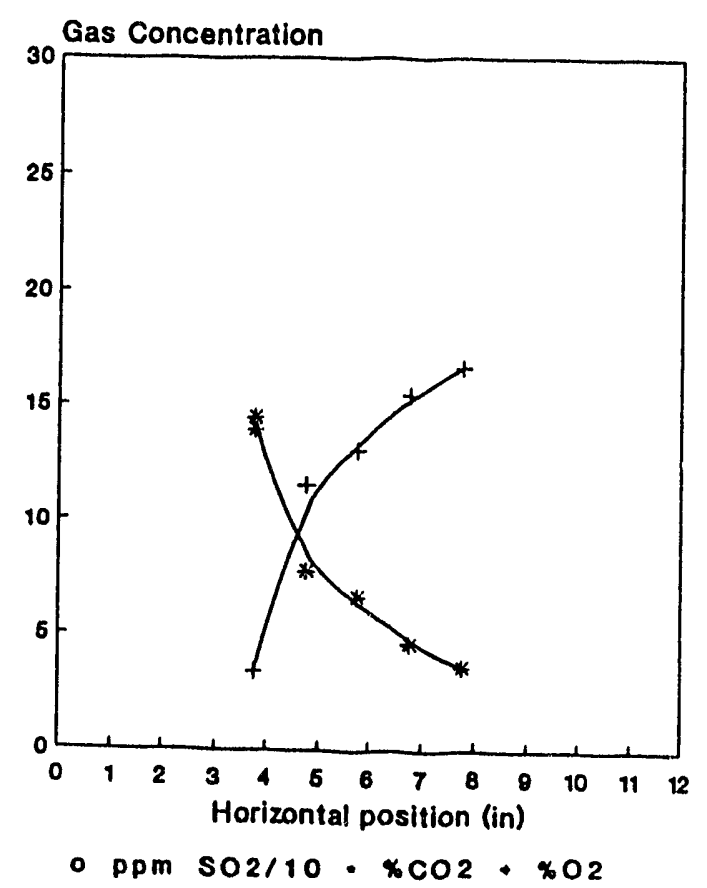

FIG. 7.30 


\section{Run: $\quad$ 12-19-91 Firing Conditions:}

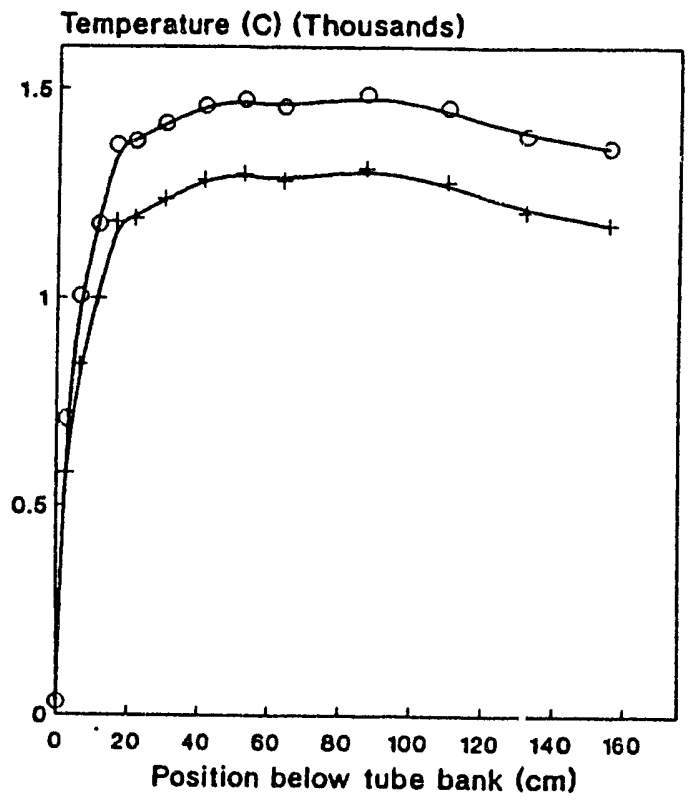

- Wall T O Calculated Flamo T
Coal $11 \quad E X O=-29.1 \%$ $14.6 \mathrm{lb} / \mathrm{hr} \quad 22.8 \mathrm{scfm}$ HORIZONTAL TEMPERATURE PROFILE AT PORT 1

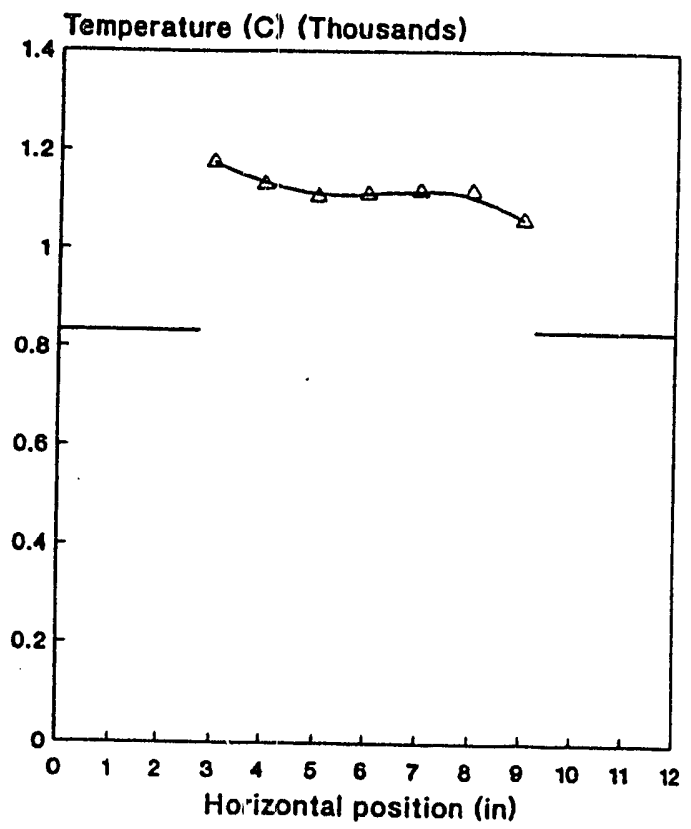

HORIZONTAL PROFILE AT PORT 2

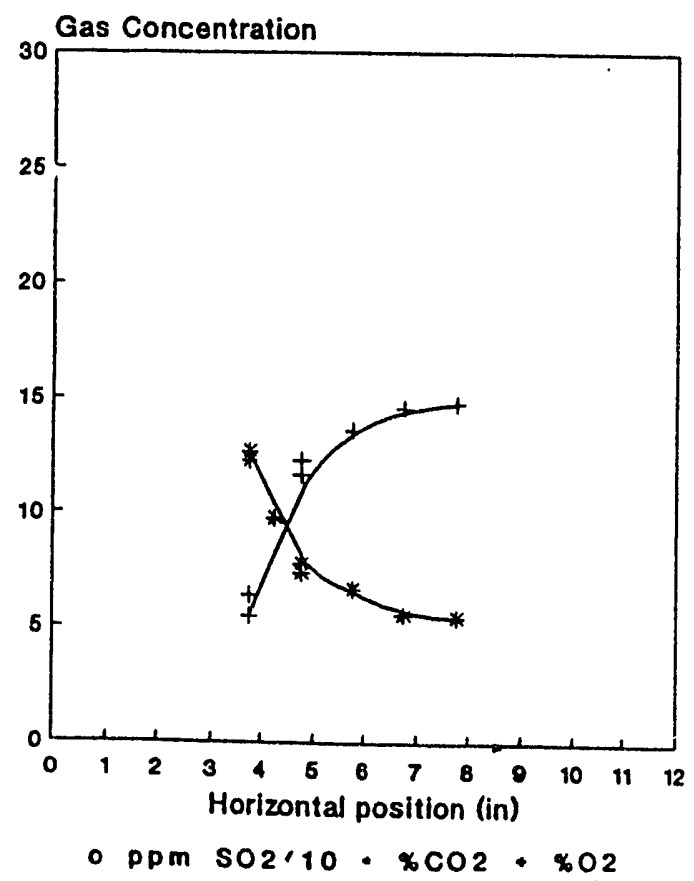

FIG. 7.31 
Run: 12-19-91 Firing Conditions:

\section{REACTION EFFICIENCY FROM PROXIMATE ANALYSIS}

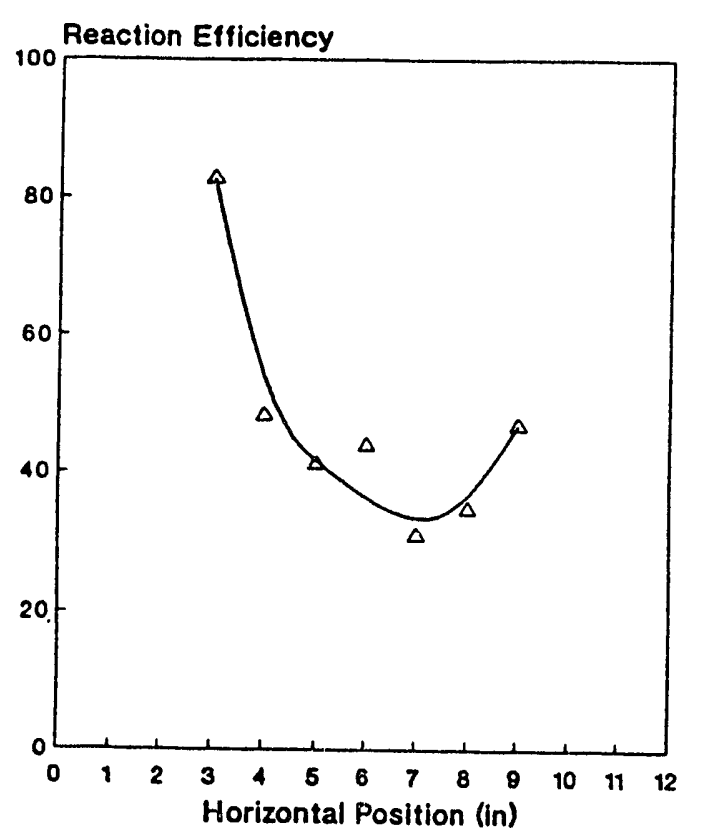

Coal $11 \quad E X O=-29.1 \%$ $14.6 \mathrm{lb} / \mathrm{hr} \quad 22.8 \mathrm{scfm}$

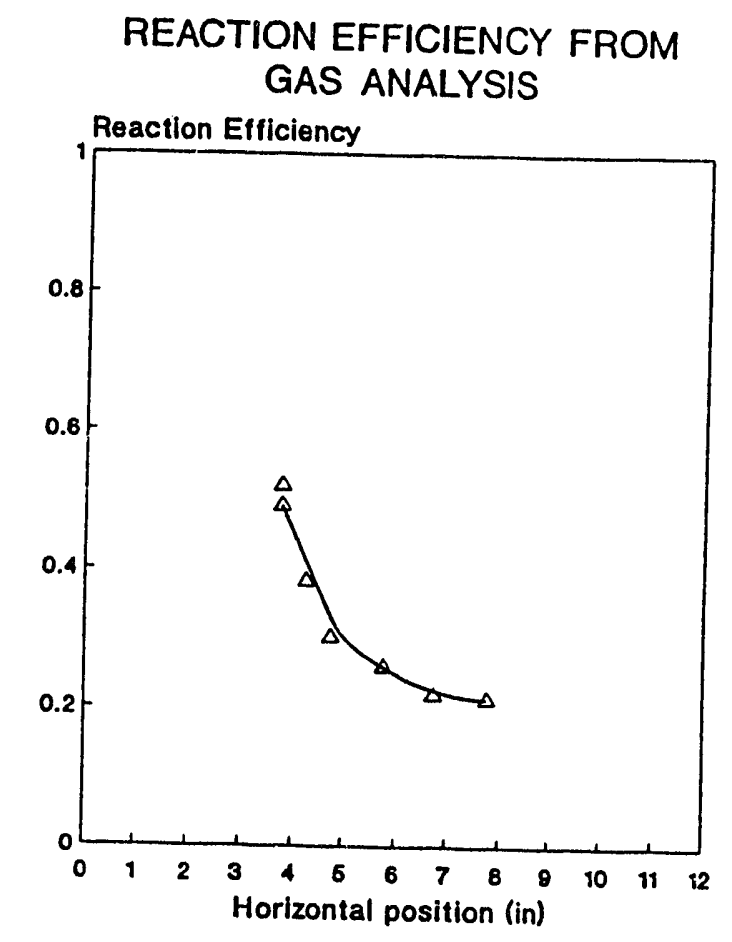

\section{REACTION EFFICIENCIES}

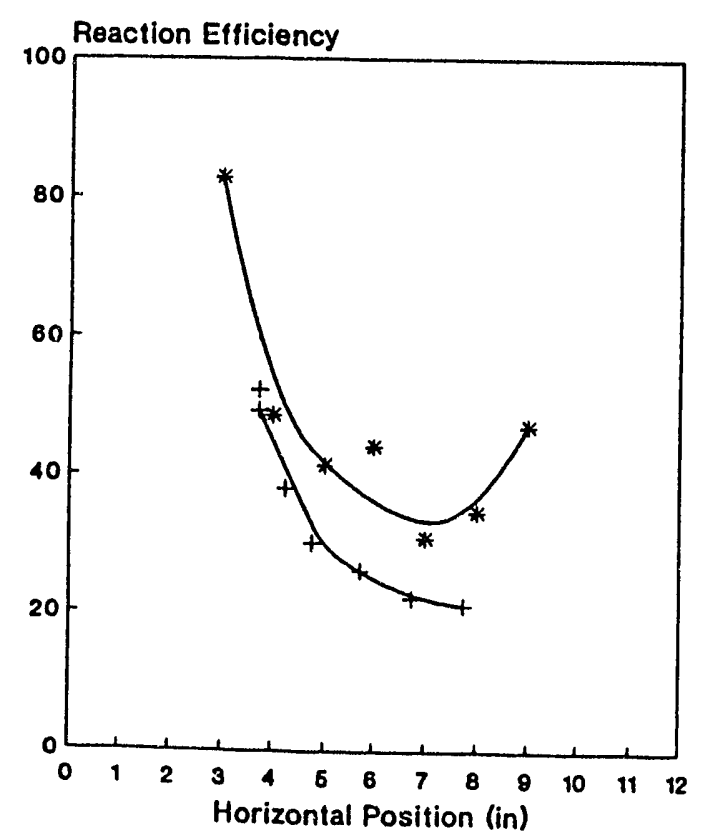

FIG. 7.32 


\section{Run: $\quad 12-21-91 \quad$ Coal $11 \quad$ EXo $=-1.9 \%$ Firing Conditions: $\quad 13.5 \mathrm{lb} / \mathrm{hr} \quad 29.7 \mathrm{scfm}$}

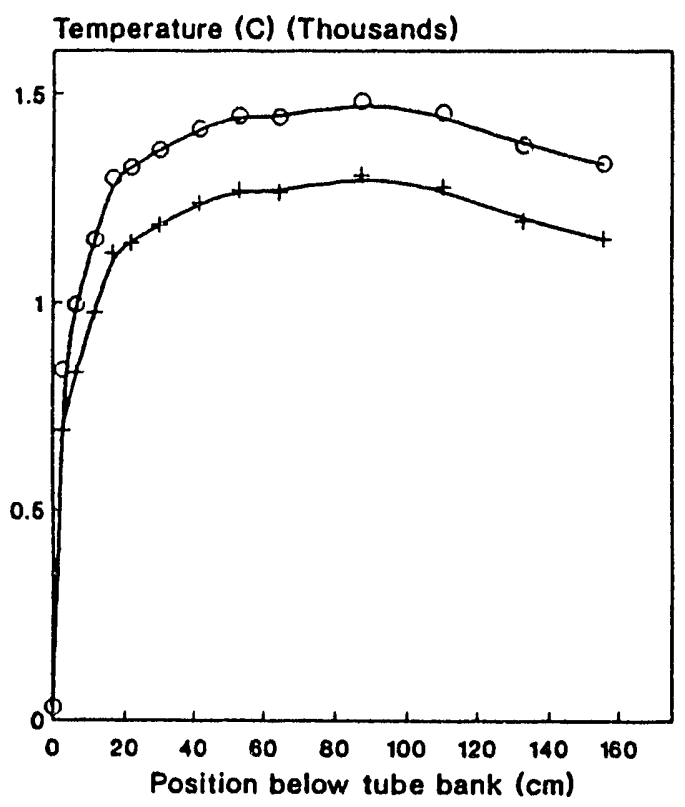

- Wall $r$
HORIZONTAL PROFILE AT PORT 2

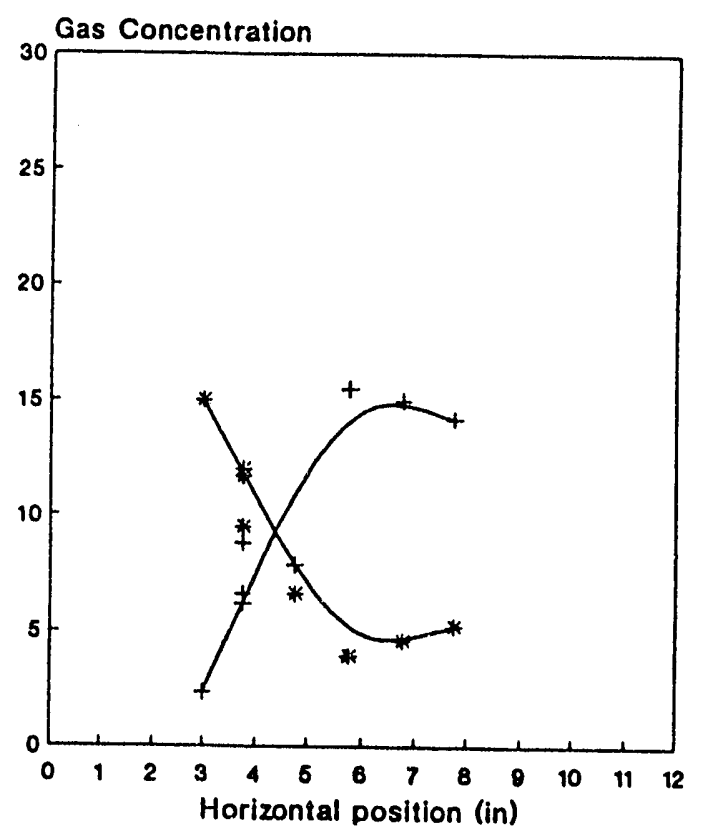

- ppm s02/10. xCO2+x02

FIG. 7.33 

Run: $\quad 12-21-91$
Coal 11
$E X O=-9.3 \%$
Firing Conditions:
$14.6 \mathrm{Ib} / \mathrm{hr}$
29.7 scfm

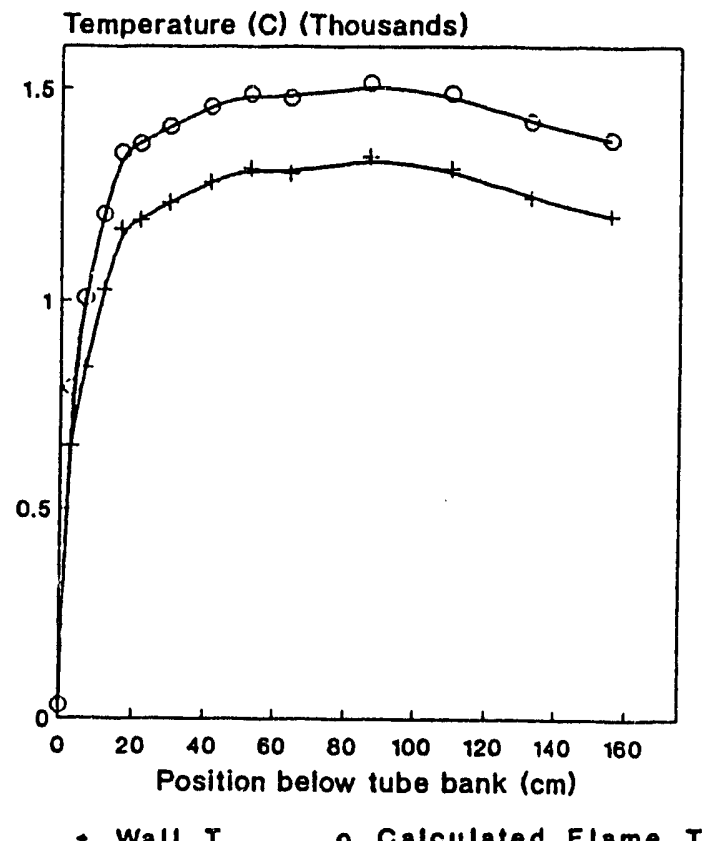

GAS CONCENTRATION AT PORT 1

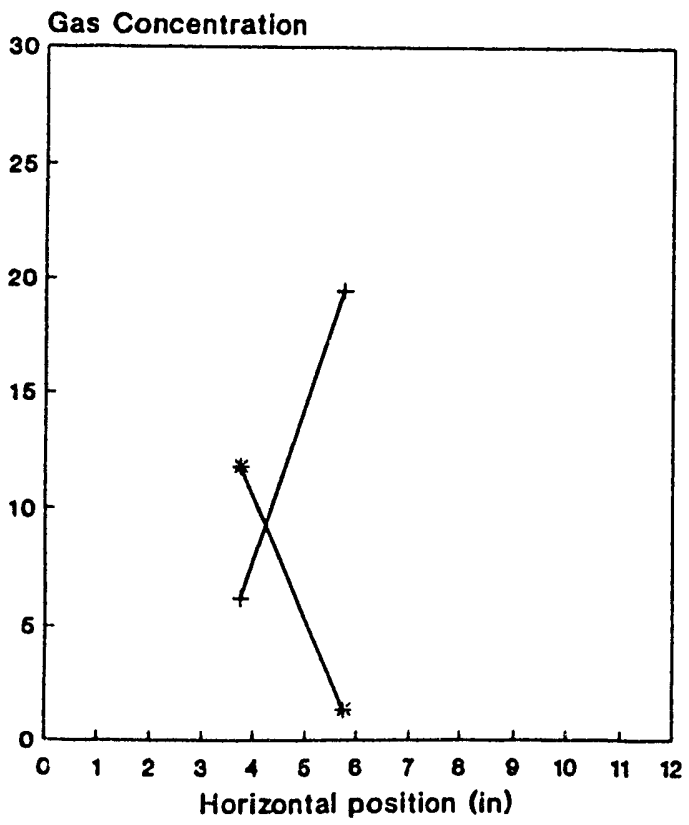

- ppm SO2/10 * $8002+\% 02$
GAS CONCENTRATION AT PORT 2

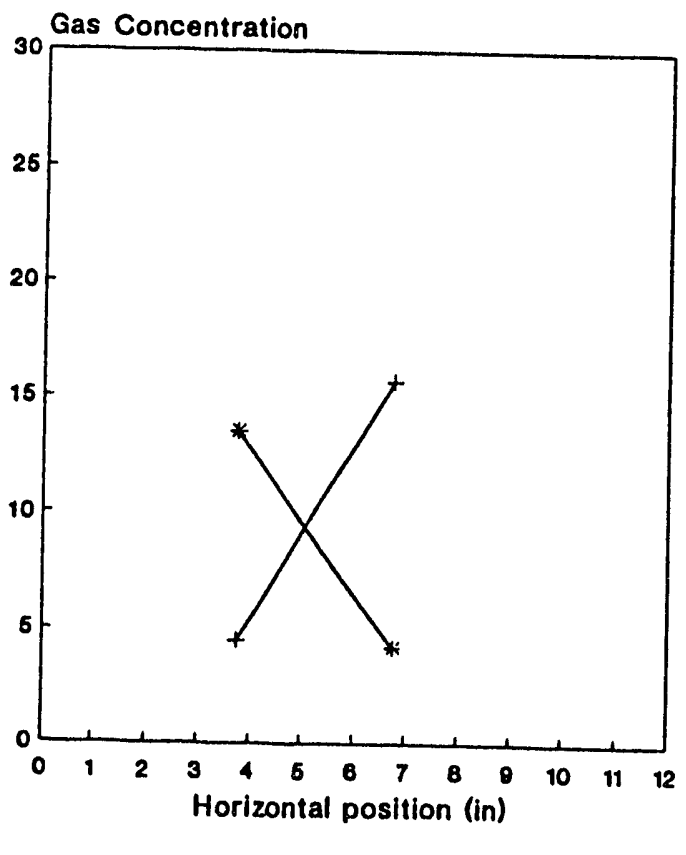

- ppm SO2/10 *xCO2 + $x_{02}$

FIG. 7.34 
$\begin{array}{lcll}\text { Run: } & 12-21-91 & \text { Coal } 11 & \text { EXo }=-18.1 \% \\ \text { Firing Conditions: } & 10.0 \mathrm{lb} / \mathrm{hr} & 18.4 \mathrm{scfm}\end{array}$

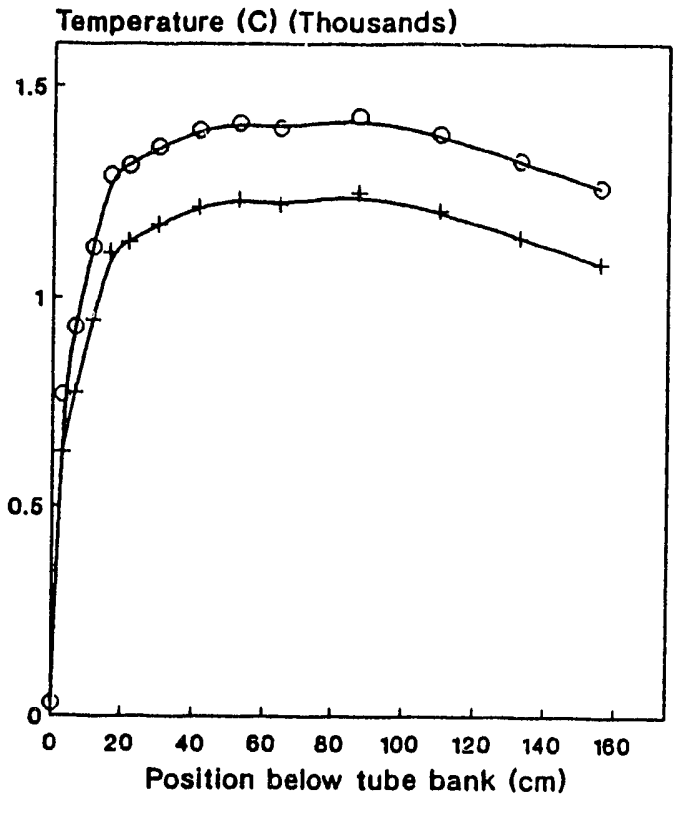

HORIZONTAL PROFILE AT PORT 2

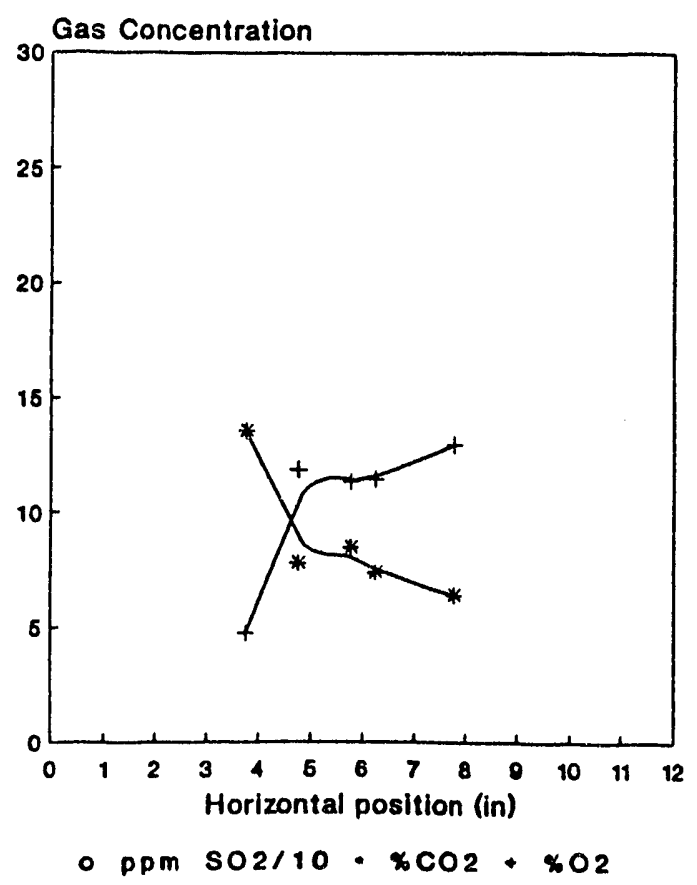

HORIZONTAL PROFILE AT PORT 9

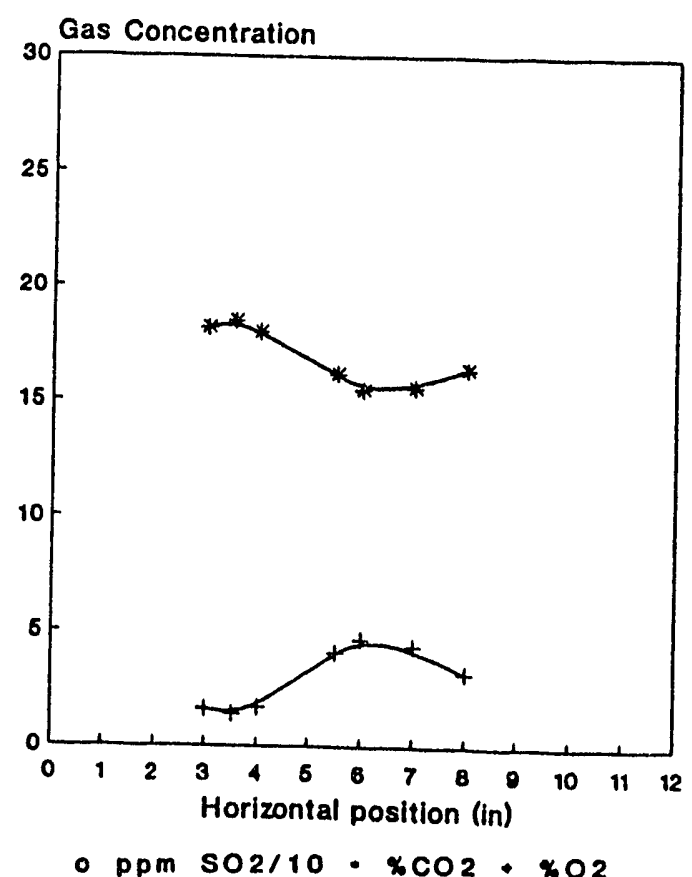

FIG. 7.35 
Run: $\quad 12-31-91$

Firing Conditions:

$18.9 \operatorname{scfm}$, EXo $=-15.3 \%$

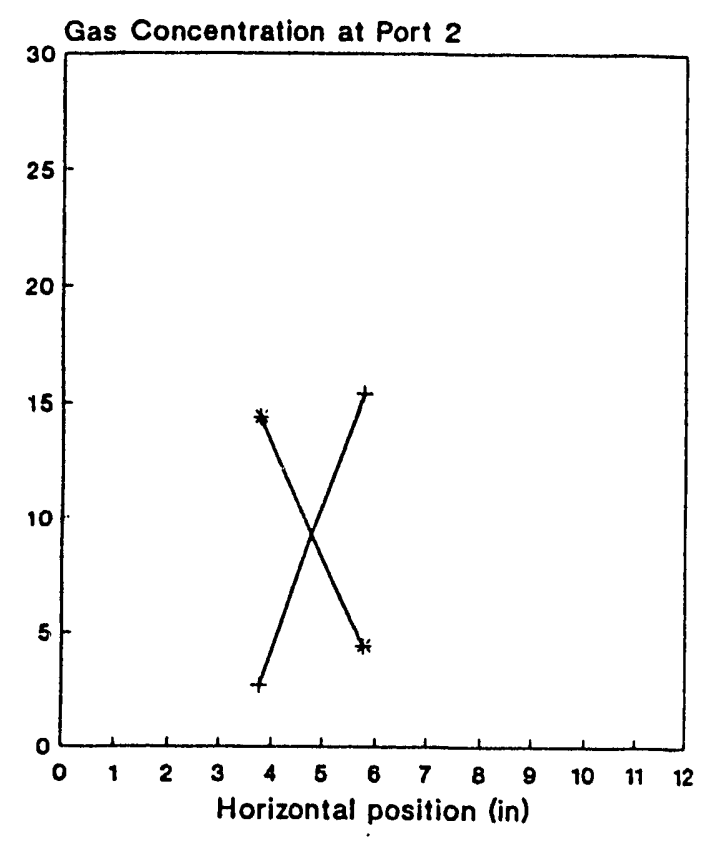

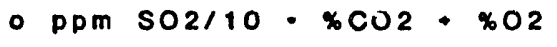

$16.4 \operatorname{scfm}, E X o=-26.8 \%$

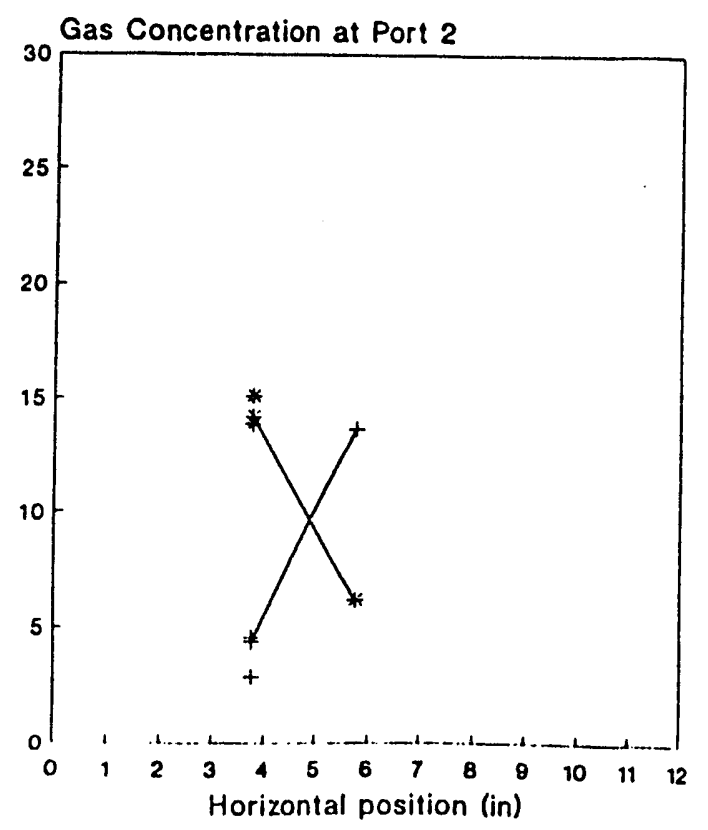

- ppm SO2/10 *x $\mathrm{CO}_{2} \cdot x_{02}$
Coal 11

$10.0 \mathrm{lb} / \mathrm{hr}$

17.5 scfm, EXo $=-21.6 \%$

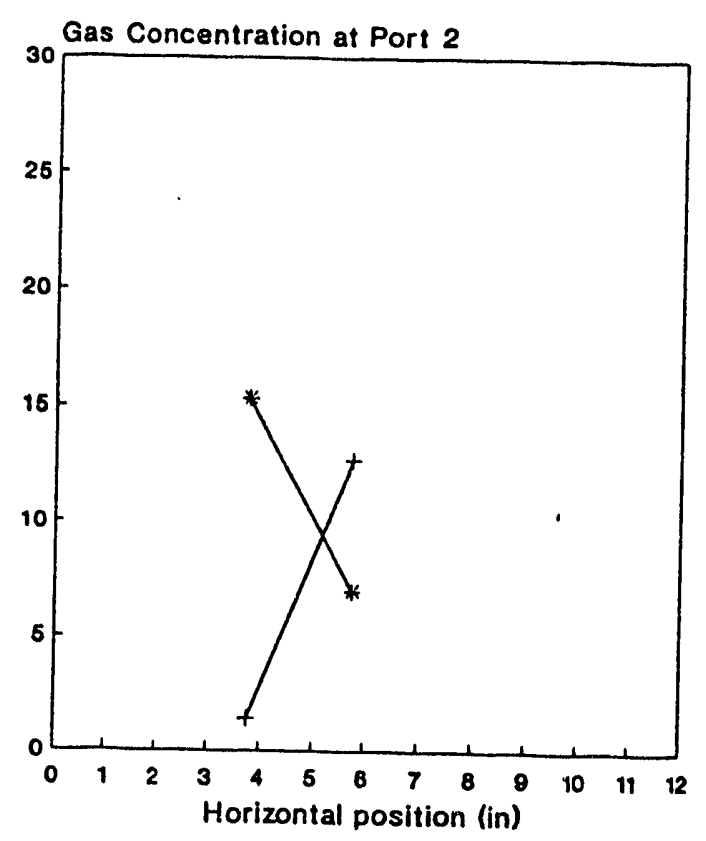

- ppm SO2/10 $* x_{0 O 2}+x 02$

11.5 scfm, EXo $=-48.7 \%$

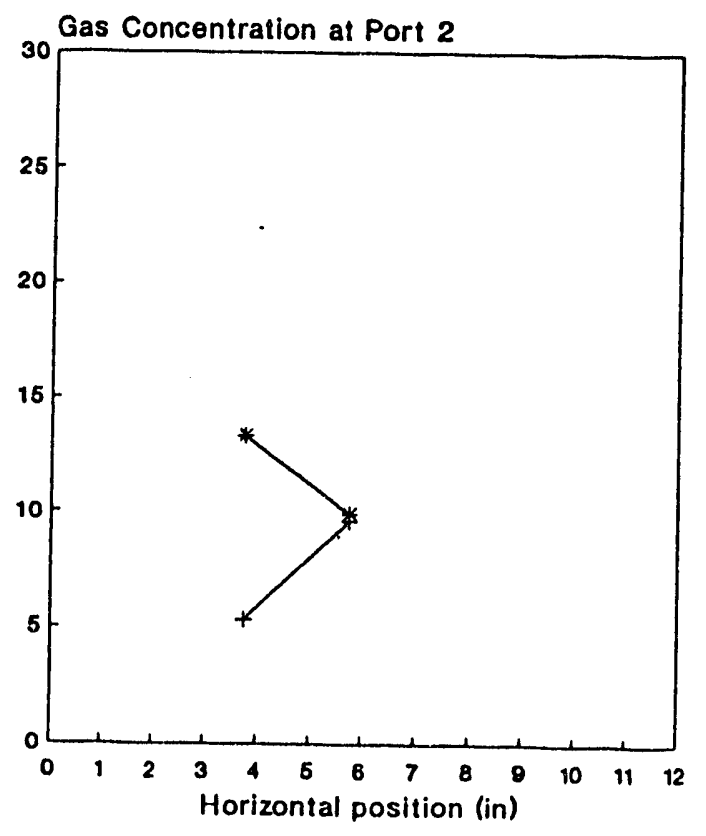

- ppm SO2/10 *xCO2 * xO2

FIG. 7.36 
Run: 1- 4-92 Firing Conditions:

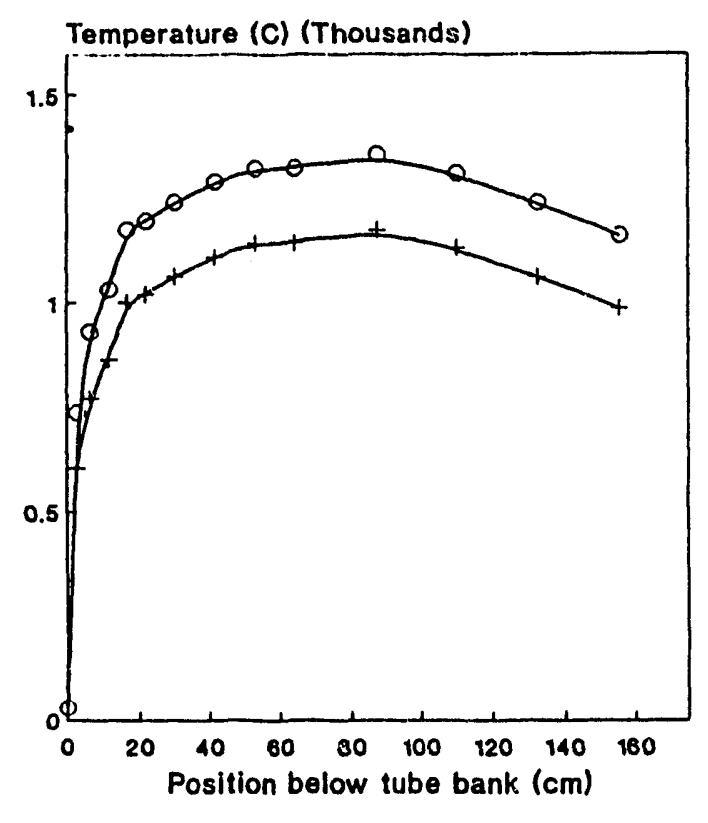

- Wall T o Calculated Flame T
Coal $11 \quad E X O=-17.7 \%$ $10.0 \mathrm{lb} / \mathrm{hr} \quad 18.8 \mathrm{scfm}$

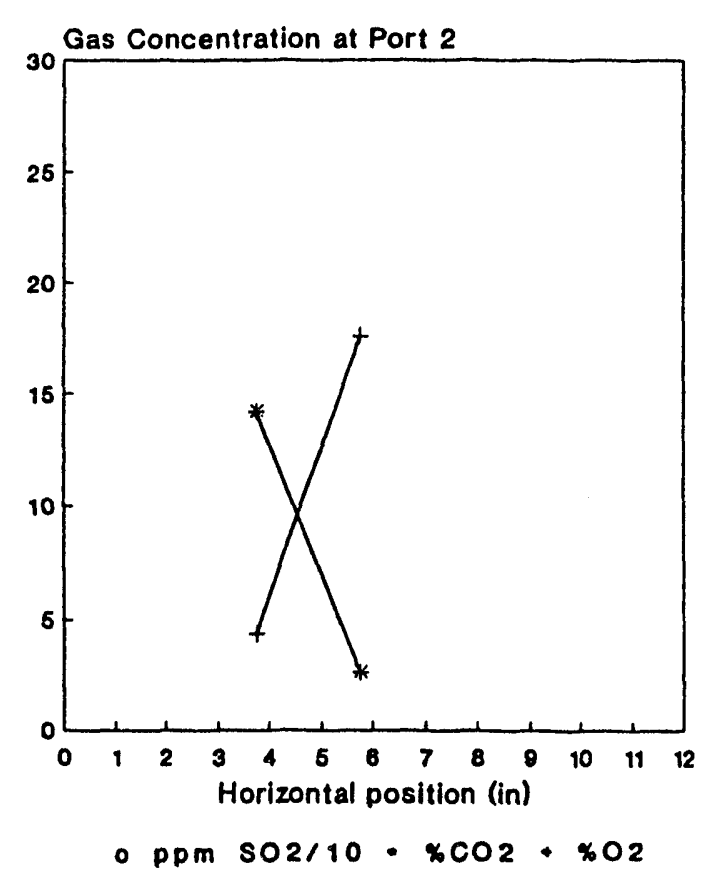

FIG. 7.37 
Run: $\quad 1-8-92$ Firing Conditions:
Coals 11,12 EXo $=-10.9 \%$ $9.8 \mathrm{lb} / \mathrm{hr} \quad 19.5 \mathrm{scfm}$

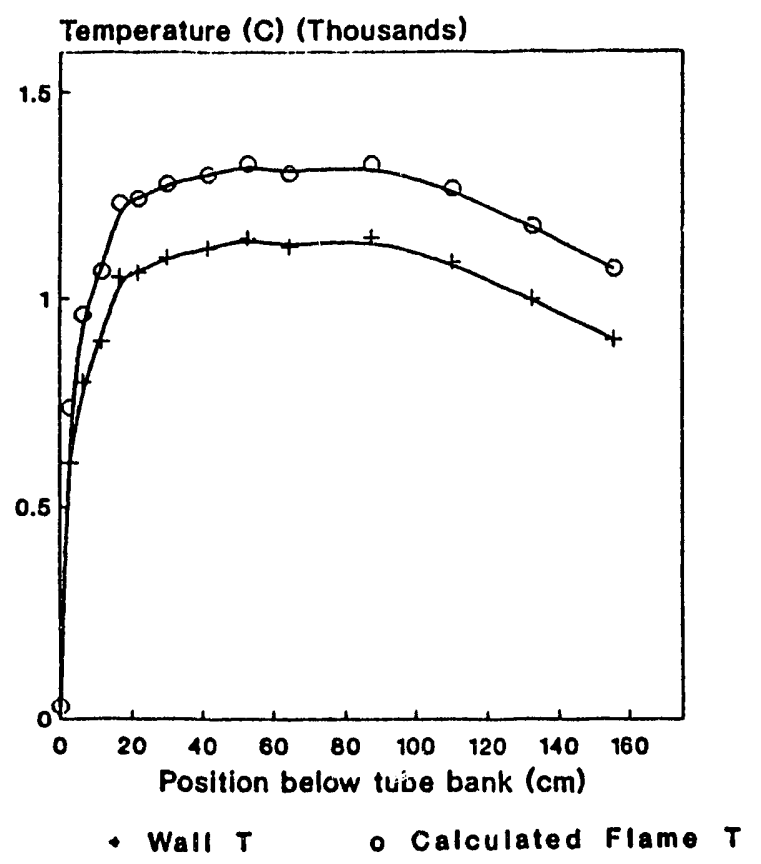

HORIZONTAL PROFILE AT PORT 2

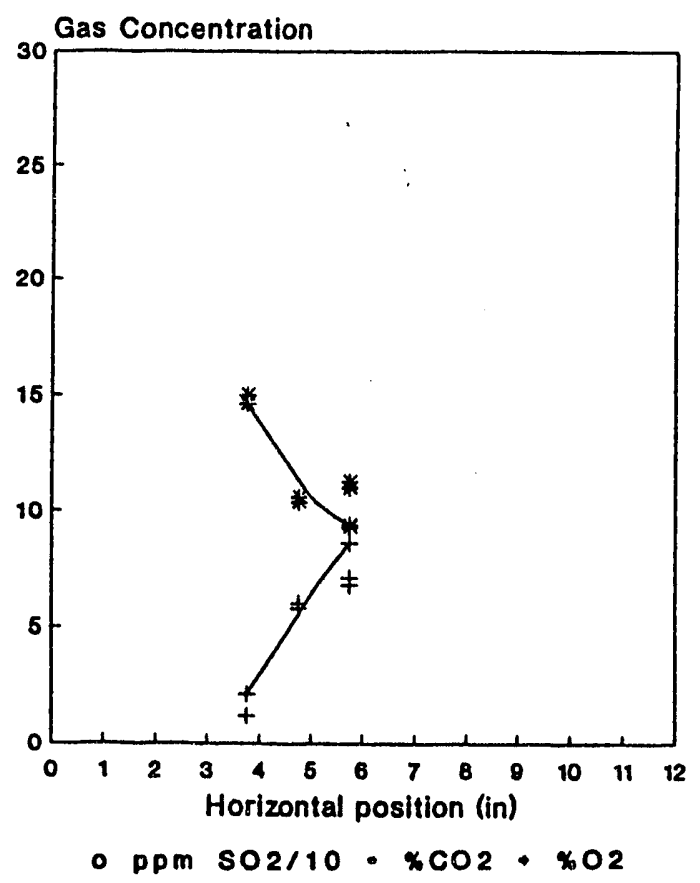

FIG. 7.38 
Run: $\quad 1-8-92$ Firing Conditions:
Coal 12

$9.8 \mathrm{lb} / \mathrm{hr}$
$E X o=-10.9 \%$

$19.5 \mathrm{scfm}$

HORIZONTAL PROFILE AT PORT 2
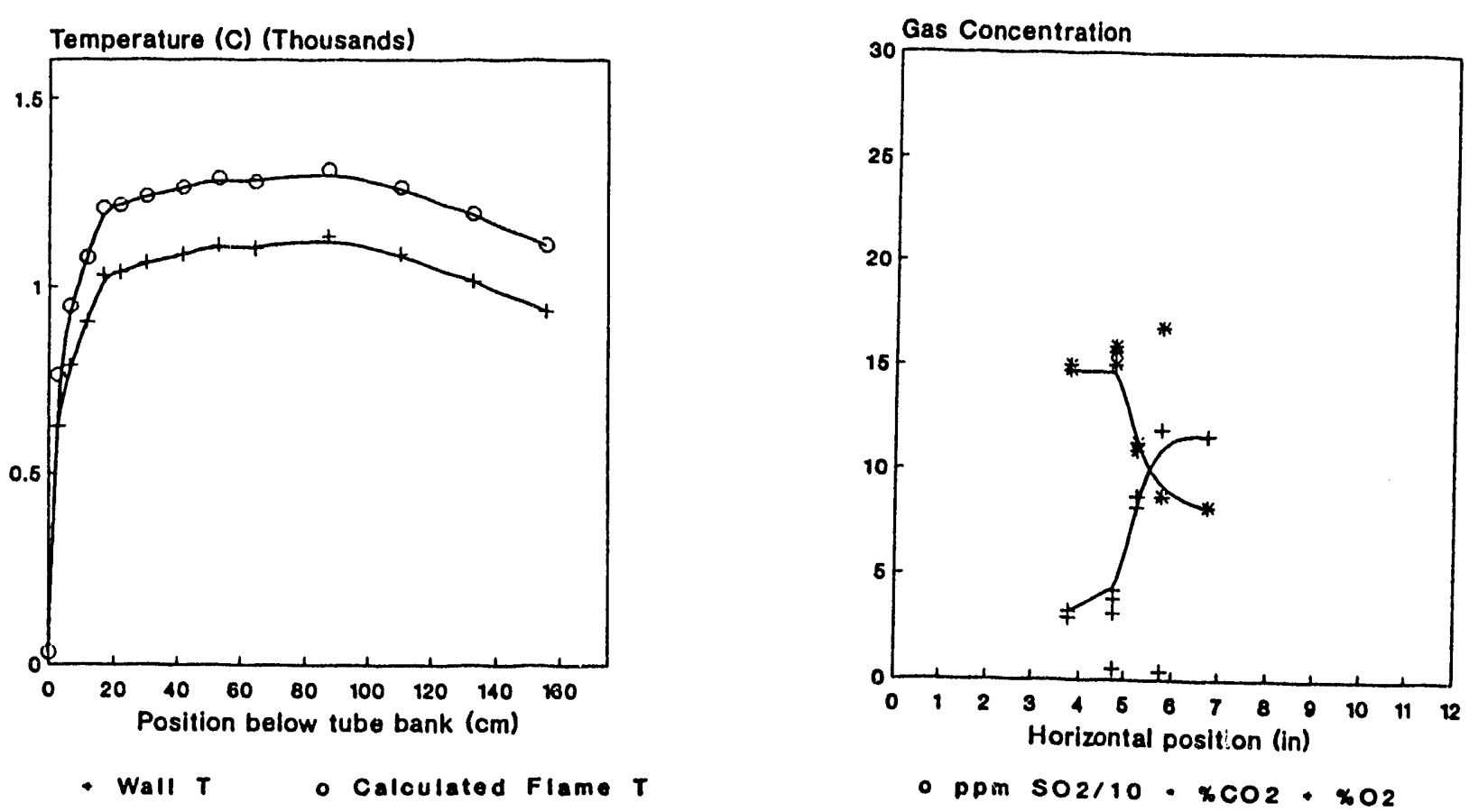

FIG. 7.39 
Run: $\quad 1-8-92$

Firing Conditions:

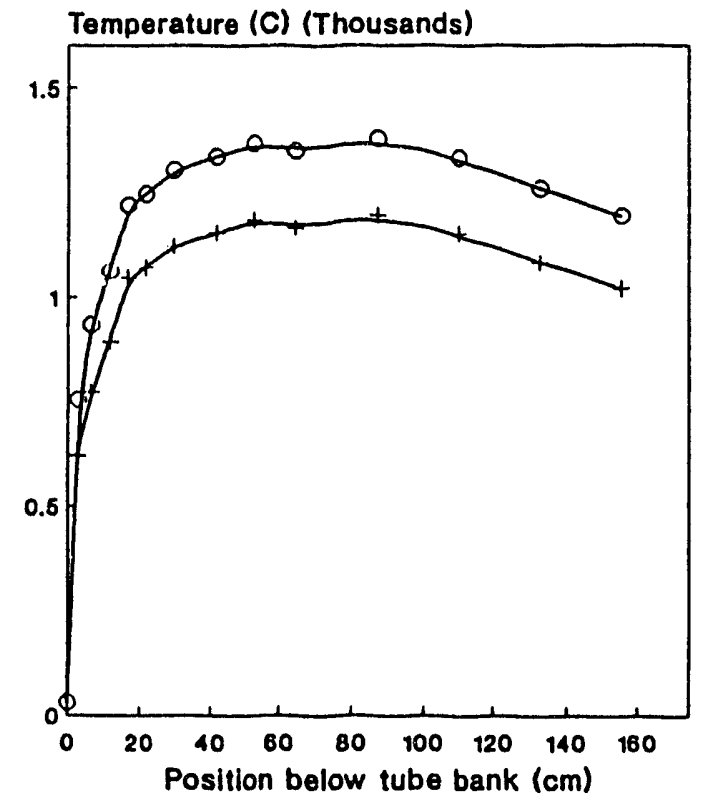

- Wall T
Coal 12 $11.0 \mathrm{lb} / \mathrm{hr}$
$E X o=-20.6 \%$ $19.5 \mathrm{scfm}$

GAS CONCENTRATION AT PORT 2

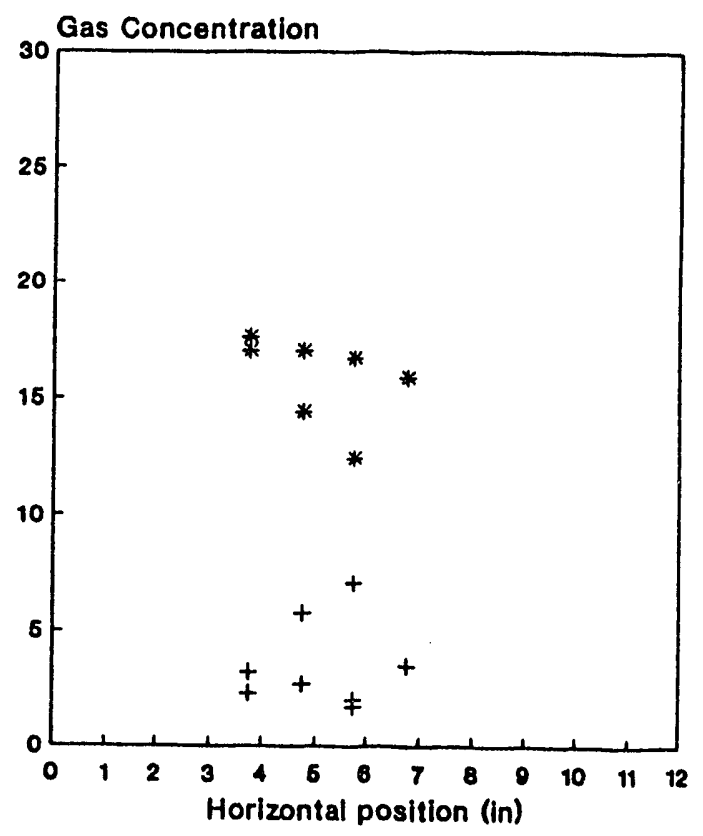

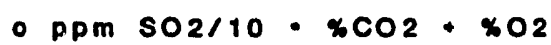

FIG. 7.40 

Run: $\quad$ 1- 8-92
Coal 12
$E X O=-11.1 \%$
Firing Conditions:
$14.5 \mathrm{lb} / \mathrm{hr}$
$28.7 \mathrm{scfm}$

GAS CONCENTRATION AT PORT 2
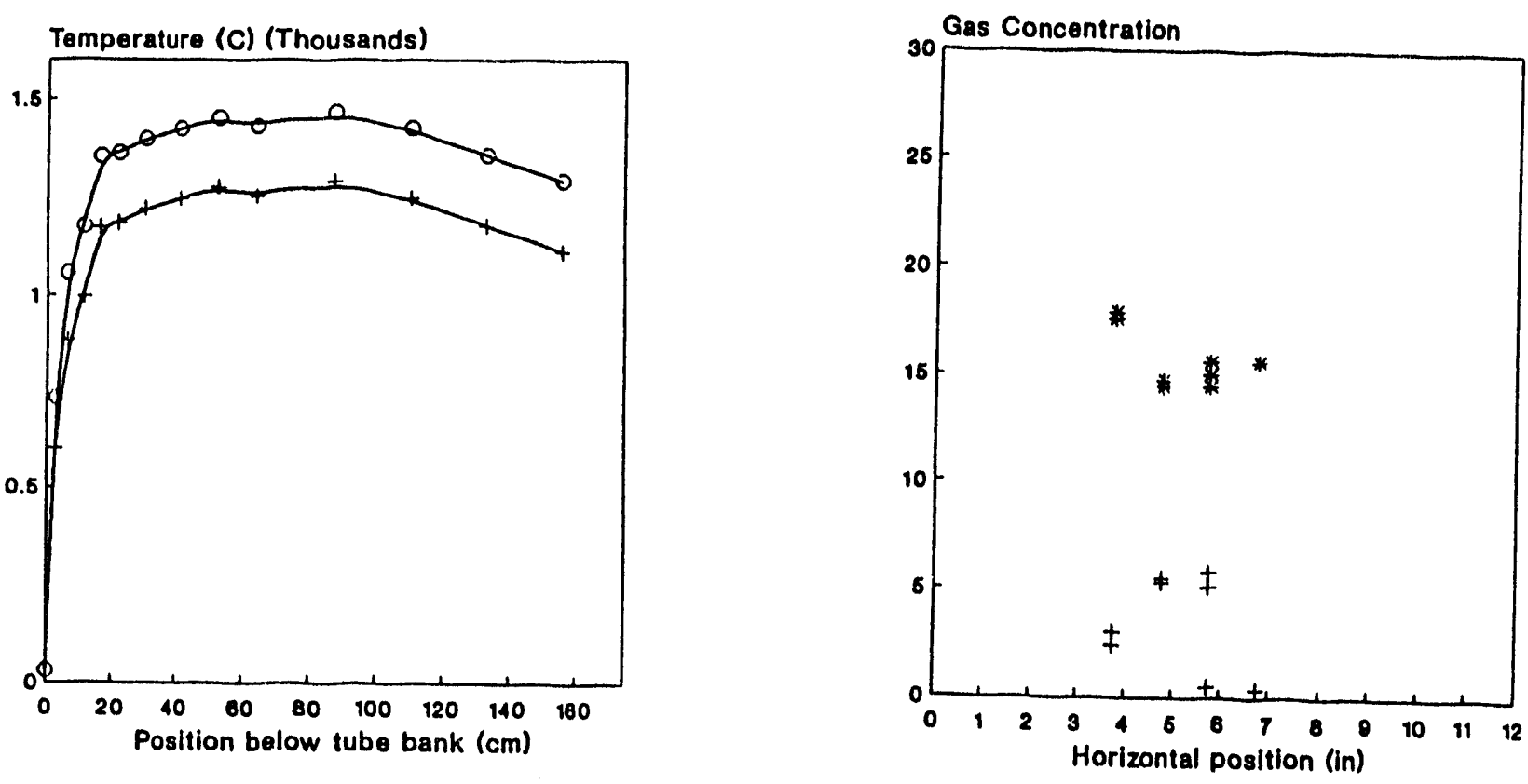

- Wall T

- Calculated Flame T

- Ppm $802 / 10 \cdot x \mathrm{CO} \cdot x 02$

FIG. 7.41 
Run: 1-14-92

Firing Conditions:

\section{GAS CONCENTRATIONS AT PORT 2}

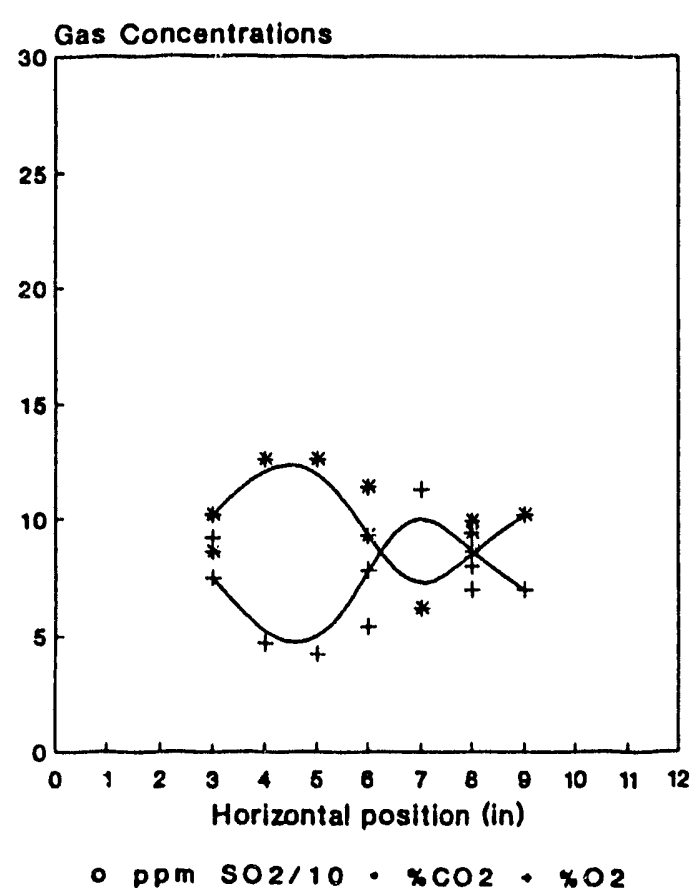

GAS CONCENTRATIONS AT PORT 5

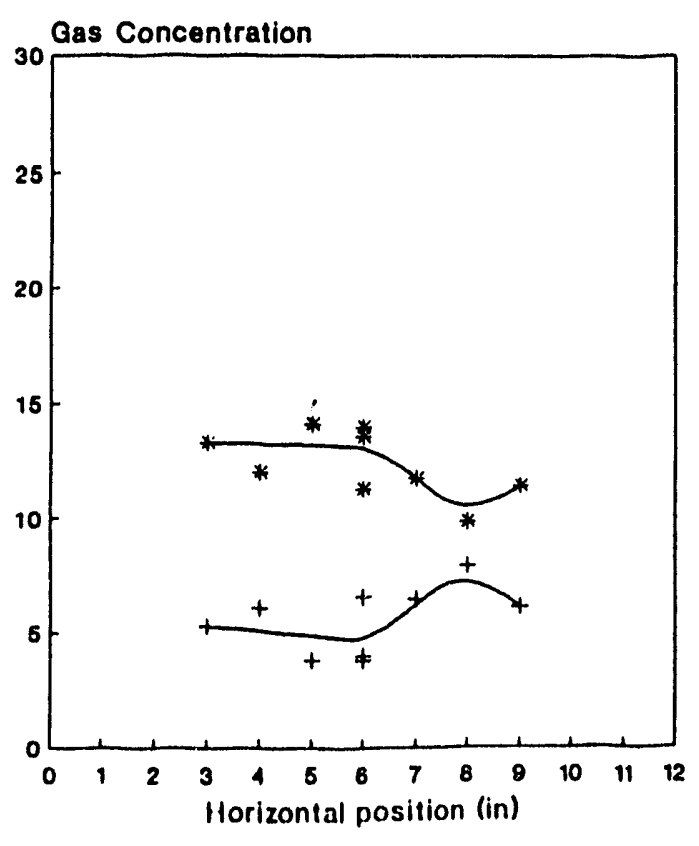

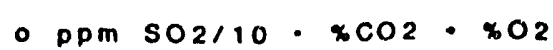

Coal $12 \quad E X O=-12.6 \%$ $9.4 \mathrm{lb} / \mathrm{hr} \quad 18.6 \mathrm{scfm}$

GAS CONCENTRATIONS AT PORT 2 DECREASED DRAFT

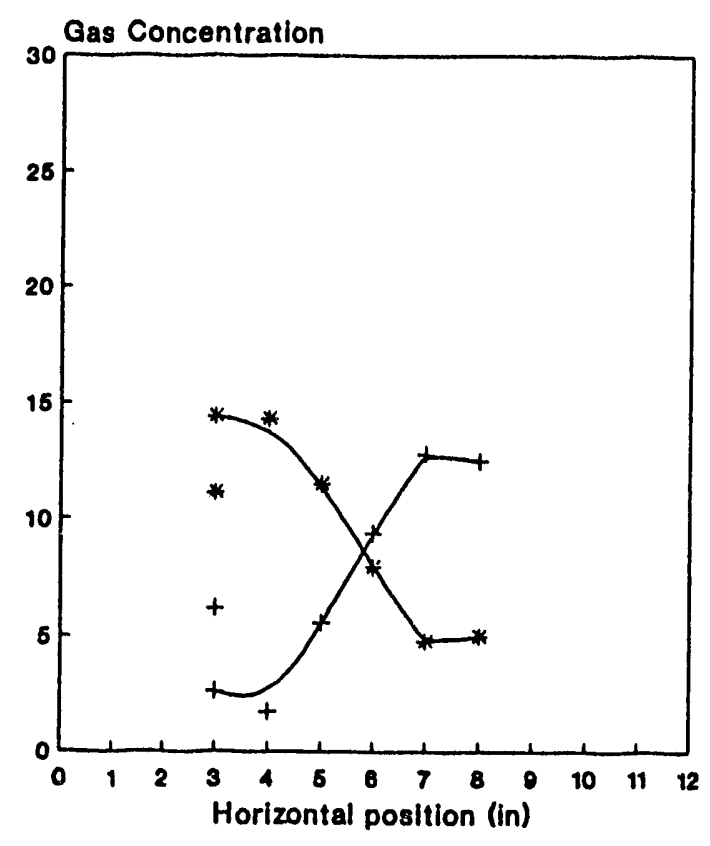

- ppm s02/10 *xCO2 * x02

GAS CONCENTRATIONS AT PORT 8

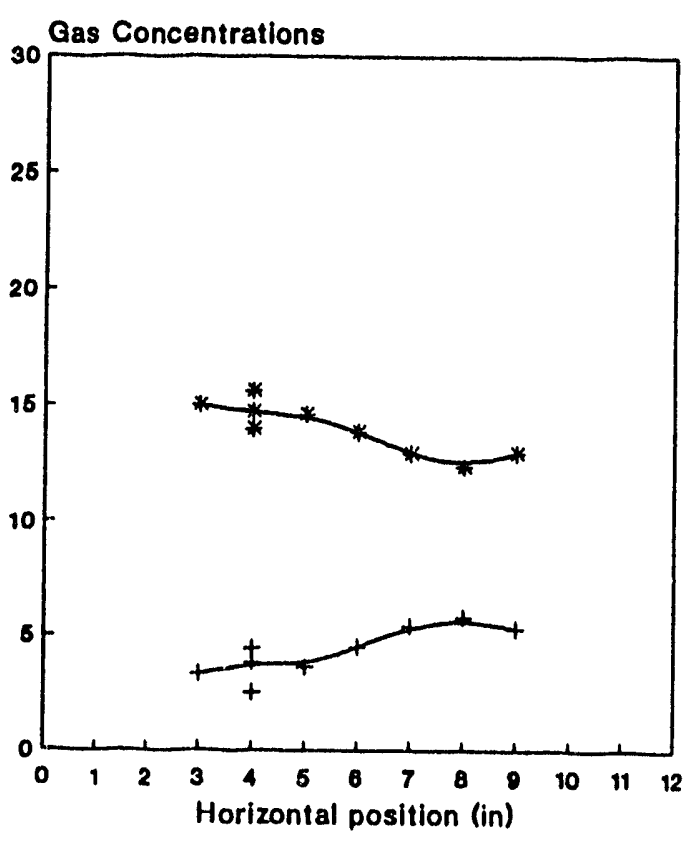

- ppm s02/10.xCO2,x02

FIG. 7.42 


\section{Run: $\quad 1-14-92 \quad$ Coal 12}

$11.0 \mathrm{lb} / \mathrm{hr}, 19.4 \mathrm{scfm}$, EXo=-22.4\%

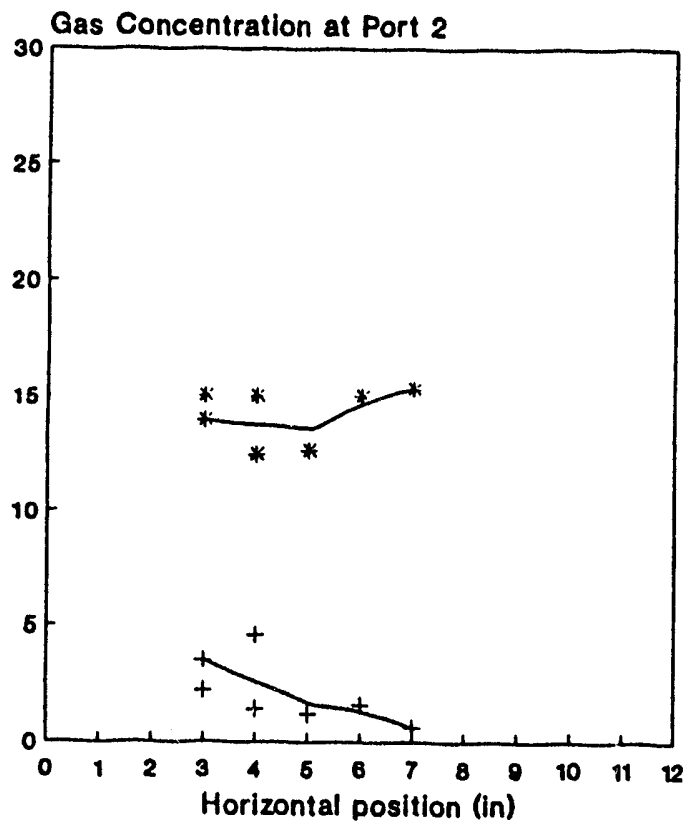

- ppm SO2/10. xCO2 402
$9.4 \mathrm{lb} / \mathrm{hr}, 20.7 \mathrm{scfm}, \mathrm{EXO}=-2.9 \%$

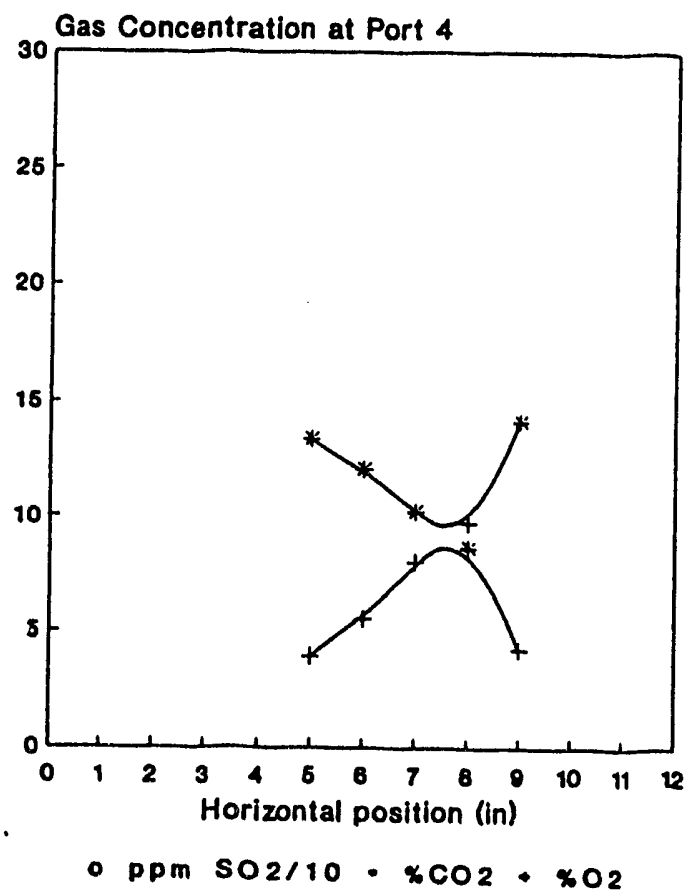

FIG. 7.43 
Run: $\quad 2-14-92$

Firing Conditions:

GAS CONCENTRATION 3.5"

BELOW TUBE BANK

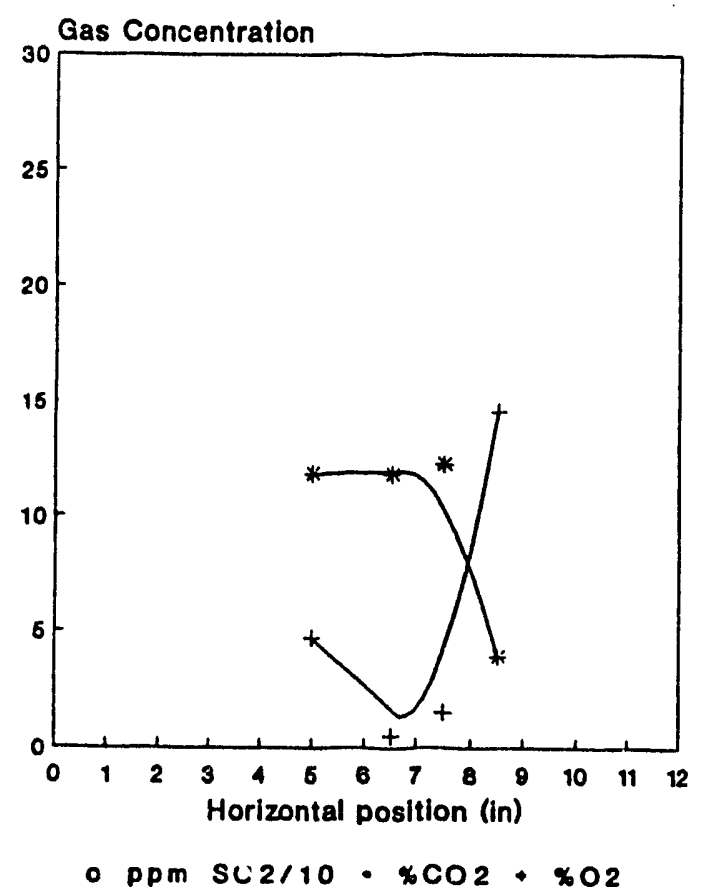

Coal 12

$E X_{0}=-52.0 \%$ $16.3 \mathrm{lb} / \mathrm{hr} \quad 17.3 \mathrm{scfm}$
GAS CONCENTRATION 17* BELOW TUBE BANK

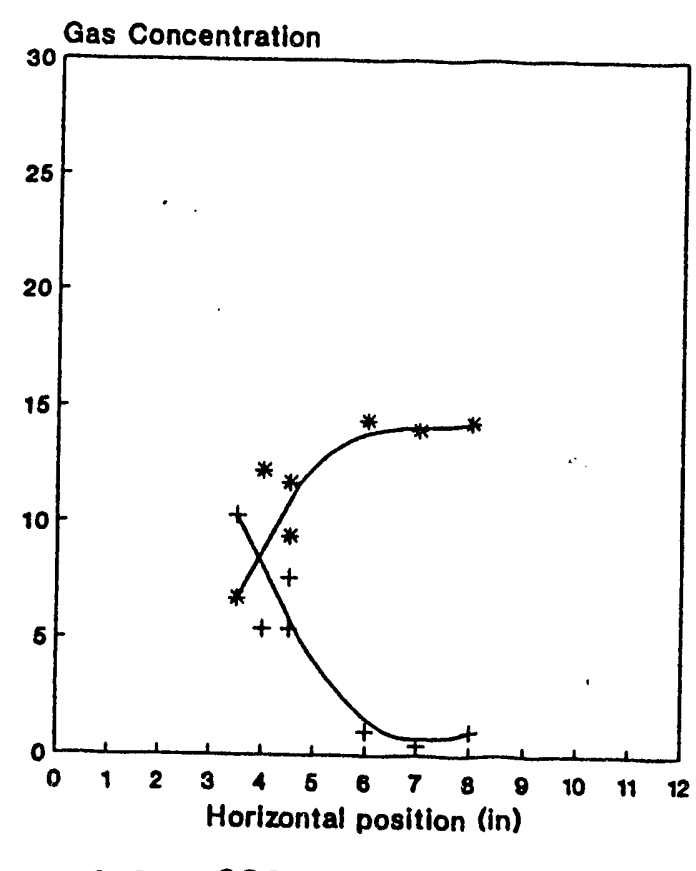

- ppm SO2/10 * xCO2 * 402

FIG. 7.44 


\section{EVALUATION AND CONCLUSIONS}

The work described in tnis Report includes development of theoretical modelling of combustion of pulverised coal combustion in one-dimensional flames, and experimental measurements in a unique furnace designed to simulate one-dimensional flames. The experimental work involved combustion measurements on 14 different coals during which about 7500 lb (nearly 4 tons) of coal were burned under 80 to 90 different firing conditions in about 50 different experimental runs, ard the complete data base contains something in excess of 45000 data entries.

The objective was to predict, and to verify prediction by experiment as far as possible, the structure of the combustion profiles and sulfur re?ease profiles in the flame, and also to investigate the change in the sulfur profile on addition of limestone to the coal being fired.

Execution of the project, however, was hampered by a number of unanticipated problems, most notably, that the flame structure developed in the furnace was changed from that developed in past studies using this furnace design, for reasons that are stiil not entirely certain but that woud appear to include apparently minor but, in fact, significant changes to the furnace design and structure. One such was an increase in shaft height, ostensibly to increase the time in the flame for higher accuracy in the flame measurements overall. The consequence of the design changes, nevertheless, was that the flame structure was substautially axially two-dimensional, with cone-shaped flame 
fronts that limited the ability to interpret the furnace measurements using the one dimensional model. This was a scientifically interesting but unexpected result; and it was unfortunate in that it deflected the project from its intended objectives since these could not be accomplished until the source of the changes in flame structure could be determined.

There were, nevertheless, a number of successful accomplishments. The first was the successful development of the one-dimensional flame model that was validated to the extent possible using prior experimental work for the purpose. This model was also used to predict changes in the particle size distribution (PSD), and a comparison with data developed in the current work provided support for this component of the model although the flame structure was known to be not fully onedimensional: the departure from one-dimensionality in those specific experiments was not, evidently, significant. The model was also used to predict the sulfur release profile although verification of the predictions will not be possible until the problem of the experimental flame structure can be resolved although this is probably very close.

The othex major accomplishment was the mapping of the two dimensional flames, and the measurement and calculation of flame property profiles. These are now available as a data base that can be lised in validation of any extended, axially-symmetric model. These measurements included axial profiles of: temperature, velocity, combustion gases, combustion efficiency, and sulfur release; and similar measurements (though mostly 
excluding the temperatures) in horizontal profiles. The measurements were carried out for a wide range of fuel supply rates, from about $10 \mathrm{lb} / \mathrm{hr}$ to $18 \mathrm{lb} / \mathrm{hr}$, at excess air levels that ranged from $+16 \%$ to $-52 \%$.

To make these measurements, a number of unexpected operational problems were also encountered that first had to be resolved. Probably the most important of these because of the complexity of resolving it and the difficulty of establishing the cause was the too-frequent blockage of the tube-bank at the entrance to the hot section of the furnace. Two separate reasons for this were subsequently identified: the first was a small inadvertent change in design of elements of the tube bank (the sharpness of the leaders on top of the tubes) that facilitated bridging by the coal; and the second was a complication due to an unknown degree of moisture in the combustion air due to failure of the drying equipment for the university compressor. As a third complication, the coal used in most of the exploration experiments was found to have the additional predisposition to bridge even under the least adverse conditions. Consequently, it was never clear at first what were the specific cause(s) of the same problem.

An additional operational problem that again had to be identified as the result of identifying certain errors of measurement was an evident problem in accurate sampling of solids in the flame. This was particularly the case for measurement of the particle size distribution (PSD) which was found to be sensitive to the methods of sampling. This sampling problem was 
one that had to be solved first before it was possible to resolve the question of the flame structure. The solution here was the development of a water-cooled slot prope using sedimentation instead of suction for collection.

The problem of the flame structure became apparent primarily as the result of the initial measurements of sulfur release. The quantities determined in the flame using only onaxis measurements were significantly lower than expected from calculation, assuming quantitative conversion. One location for the missing sulfur was in the off-axis flow, and measurements established that this was, at least in part, evidently the case. To account for this, however, then required the assumption that the flame itself was not one-dimensional; yet, establishing this was not a simple matter. This required extensive measurements of horizontal profiles including an extensive study of the mixing behavior in the mixture supply cone; but the conclusion was that there was a two-dimensional flame pattern for reasons that have still to be established for certain.

The examination of the furnace design and operation to determine the source of the change in flame structure required most extensive investigation of a wide range of design and operational factors. This included the problem of the uniformity of both dust and air distribution across the furnace (partly the cone mixing problem), with some clear evidence that one method of coal and (direct axial) air supply did generate coal rich flow at the wall and parabolic air flow at the center. overall, however, 
these different factors were not able to account for the coneshaped flames. Accordingly, attention was then directed to the possible influence of firing conditions as the source of the change in flame type. Additionally, a further factor considered was the effect of furnace height.

The varied investigations showed clearly that one significant influence on the flame and the profile measurements was the magnitude of the furnace draft. This is controlled by injectors at the bottom of the flue riser alongside the furnace, and can be adjusted to provide a range from slightly pressure positive to pressure negative. The ideal is usually considered t) be balanced pressure at about mid-height up the furnace so that the upper section may be slightly positive and the lower section slightly negative. There are problems in both cases, however. Under negative pressure, there is air inleakage and, if this is sufficiently great, it can distort the flame property profiles both vertically and horizontally, and invalidate the results as sources for testing the theoretical model. Under positive pressure, the natural buoyancy of the hot flame column can over-ride the pressure gradient driving the gases down towards the flue exit and cause local backmix back up the furnace. This was evidently part of the source of changes in the flame behavior where buoyancy-driven vorticity at the flame front was clearly a modifying agent to the flame front in some flames at least.

To control this, in a final sequence of experiments the effect of buoyancy was reduced by reverting to a lower shaft 
furnace, of a height that had been used in the first experiments, and this did evidently eliminate the buoyancy-vortices. At the same time, the stoichiometry was increased, going substantially more fuel-rich, with some increase in firing rate, and in consequence, the appearance of the flame was much closer to that observed in the past. Most significantly, the non-linear horizontal property profiles were inverted with respect to the original, so that low oxygen at the wall became high, and high CO2 at the wall become low. At that point it was necessary to terminate the experiments, but it would appear that although the exact window of operation that produces the desired flame structure is still not fully identified, nevertheless, the margins of the required window of operation for the onedimensional flame are believed to be finally identified. Proof of this requires further work.

In conclusion we have been able to solve one of the principal problems, of modeling the flame to the point that sulfur profiles can now be predicted. Verification of the combustion model using prior data is reasonably complete, but verification of the sulfur release is not yet possible on account of the experimental problems encountered. Nevertheless, the window of operation for generation of the one-dimensional flames may now have been determined, and continuation of this work is a natural progression to develop a data base by which the sulfur release profiles and, in the future, the sulfur sorption profiles can be tested as needed for verification of the sulfur release and sorption models. 

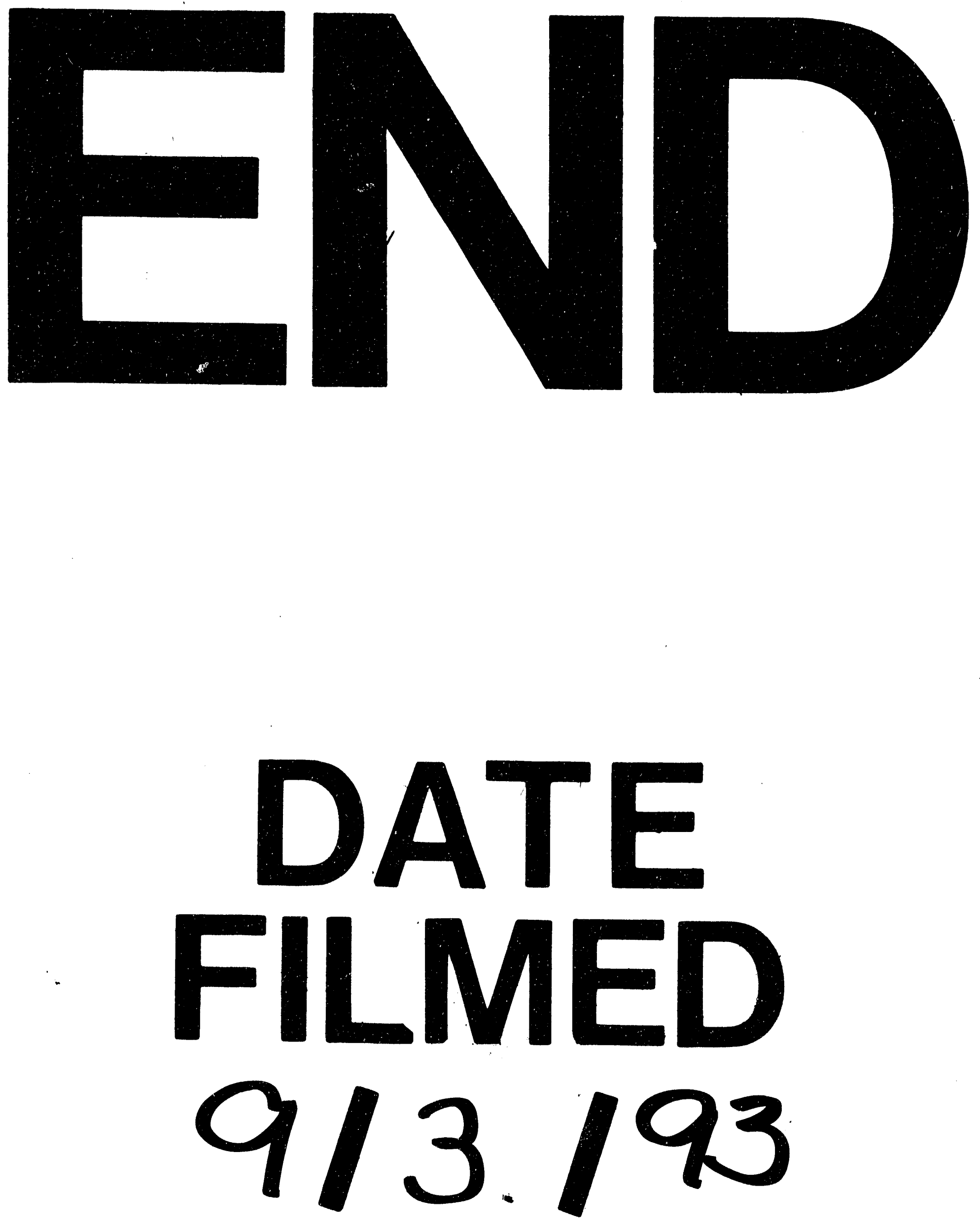
